\title{
The role of innervation during mouse embryonic myogenesis: what molecular genetics tells
}

\author{
Dissertation \\ in partial fulfilment of the requirements for the degree \\ “Doctor rerum naturalium" \\ in the Neurosciences Program \\ at the Georg August University Göttingen, \\ Faculty of Biology
}

submitted by

Chor Hoon Poh

born in

Bangkok, Thailand

Göttingen 2013 


\section{Thesis committee members}

Dr. Till Marquardt (Supervisor, Reviewer)

Developmental Neurobiology

European Neuroscience Institute, Göttingen

Prof. Klaus-Armin Nave, Ph.D. (Reviewer)

Neurogenetics

Max Planck Institute for Experimental Medicine, Göttingen

Prof. Dr. Tomas Pieler

Developmental Biochemistry

Center for Biochemistry and Molecular Biology, Göttingen

Date of thesis defense: $8^{\text {th }}$ March 2013 


\section{Declaration of Originality}

This thesis, entitled "The role of innervation during mouse embryonic myogenesis: what molecular genetics tells", contains no material which has been accepted for a degree or diploma by the University or any other institution, except by way of background information and duly acknowledged in the thesis, and to the best of my knowledge and belief no material previously published or written by another person except where due acknowledgement is made in the text of the thesis, nor does the thesis contain any material that infringes copyright.

Göttingen, February 2013

Chor Hoon Poh 


\section{List of contents}

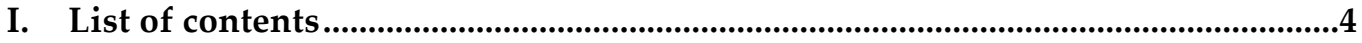

II. List of figures......................................................................................................

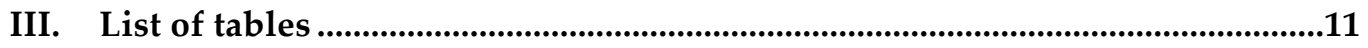

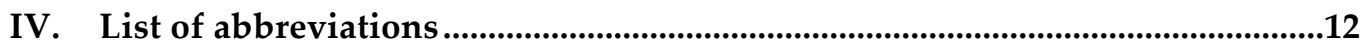

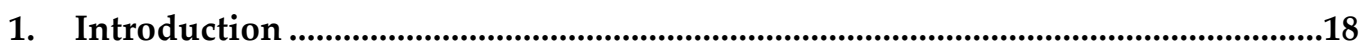

1.1. The neuromuscular system …..................................................................... 18

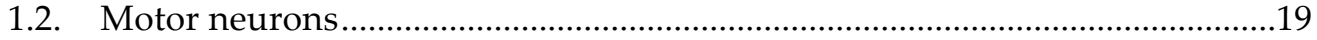

1.2.1. Motor neuron development ............................................................20

1.2.2. Motor neuron classes ...........................................................................23

1.2.3. Organization of motor neurons within the spinal cord .........................24

1.3. Generation of skeletal musculature in vertebrates ........................................25

1.3.1. Delamination and migration of muscle progenitor cells ......................25

1.3.2. Cellular events during myogenesis .................................................26

1.3.3. Molecular events during myogenesis...................................................29

1.3.4. Genetic hierarchy implicated in limb myogenesis ...............................31

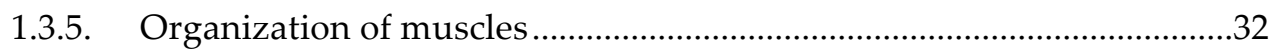

1.3.6. Types of muscle fibers arising during embryonic myogenesis .............33

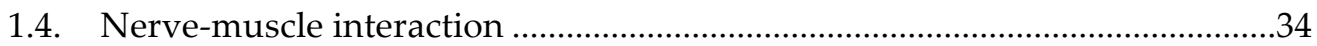

1.4.1. Dependence of muscles on innervation ............................................35

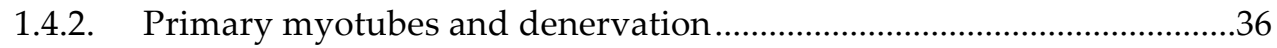

1.4.3. Influence of innervation on secondary myotubes ................................37

1.4.4. Nerve aspects that could regulate muscle development ........................38

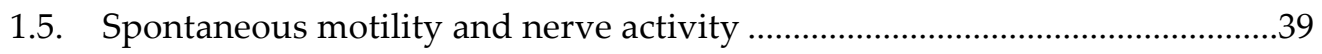

1.5.1. Evoked and spontaneous vesicular release ........................................40

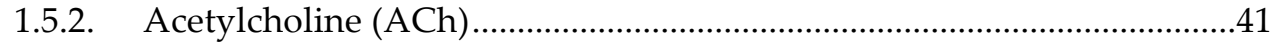




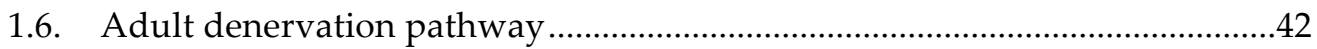

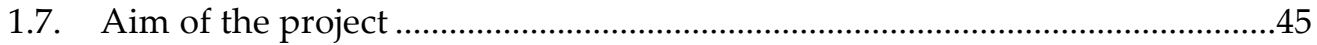

2. Materials and methods ..................................................................................................47

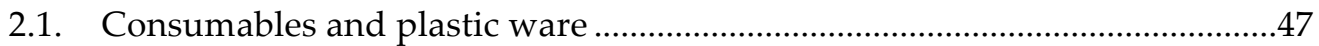

2.1.1. Antibodies ..............................................................................................

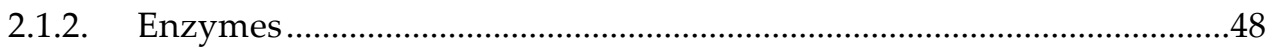

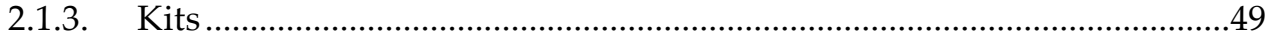

2.1.4. Chemicals and reagents ............................................................................49

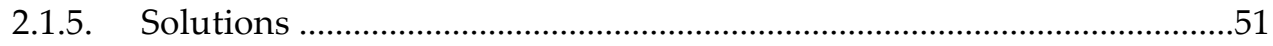

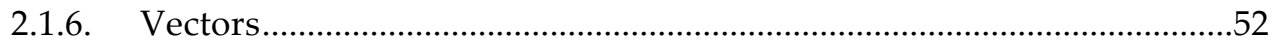

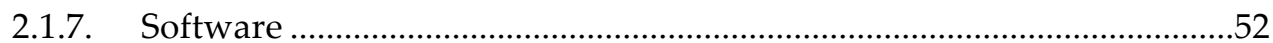

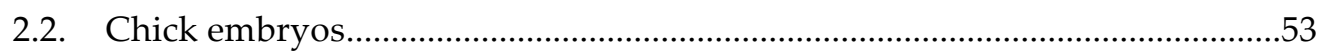

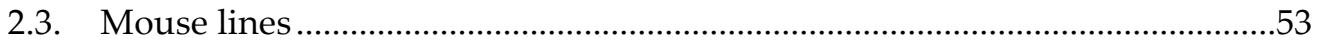

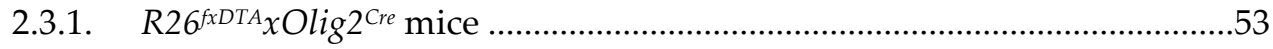

2.3.2. Isl2 ${ }^{f x D T A} x$ Olig2 $2^{\mathrm{Cre}}$ mice ……....................................................................54

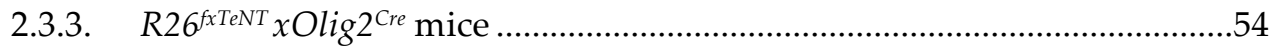

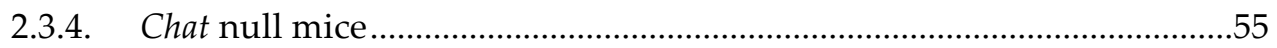

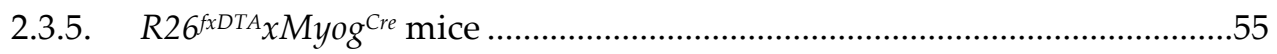

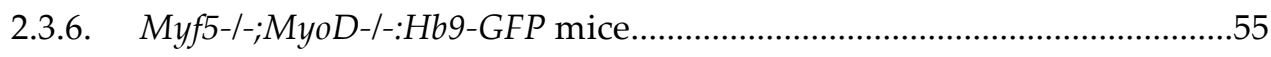

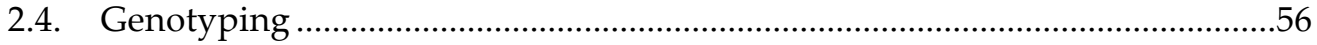

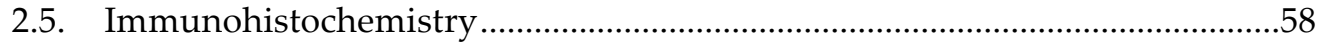

2.5.1. Immunohistochemistry - Paraffin sections ...............................................58

2.5.2. Immunohistochemistry - Frozen sections ..................................................59

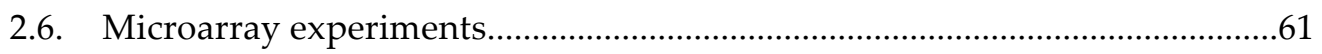

2.6.1. Sample processing for microarray experiments .......................................61

2.6.2. Hybridization ...........................................................................................61

2.6.3. Pathway analysis ......................................................................................62

2.7. RNA sequencing experiments .....................................................................62

2.7.1. Sample processing for RNA-seq experiments.......................................62 
2.7.2. Pathway analysis .......................................................................................63

2.8. Quantitative reverse-transcription PCR (qPCR) ............................................63

2.9. Electron microscopy experiments .......................................................................64

2.9.1. High pressure freezing and Freeze substitution.......................................64

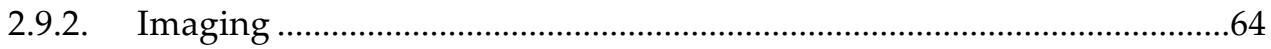

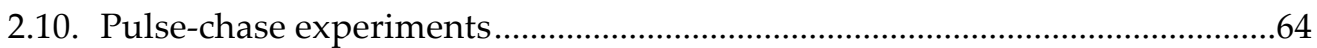

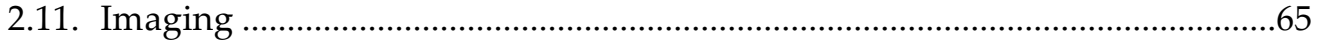

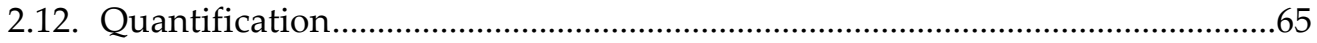

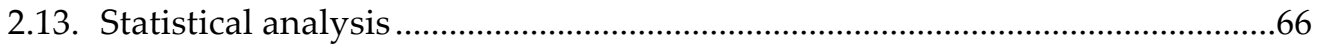

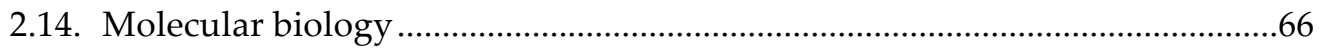

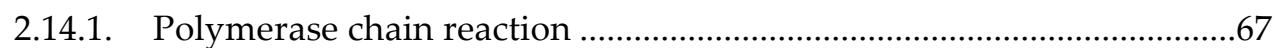

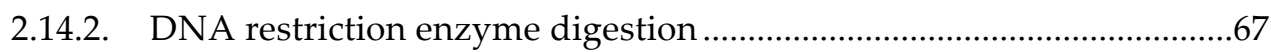

2.14.3. Alkaline phosphatase treatment ..............................................................67

2.14.4. Agarose gel electrophoresis ......................................................................67

2.14.5. DNA purification from agarose gels .........................................................68

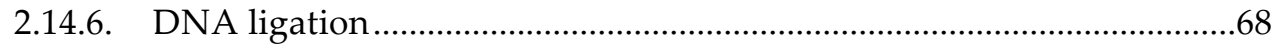

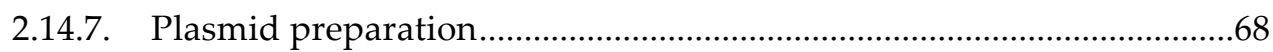

2.14.8. DNA and RNA concentration measurements .......................................69

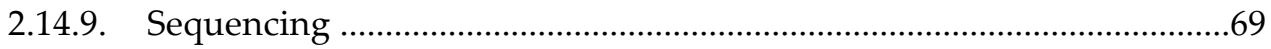

2.14.10. Preparation of chemically competent E.coli ..............................................69

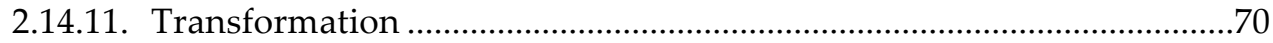

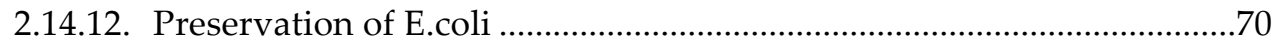

2.14.13. RNA extraction ................................................................................

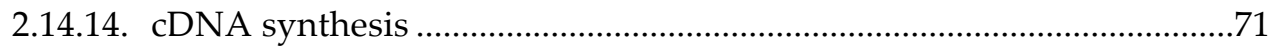

2.15. P2TK-MLC-V5-MCS-2A-eGFP plasmid construction.........................................71

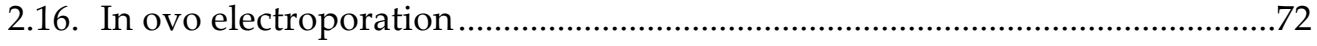

3. Results..........................................................................................................................74

3.1. Creating aneural muscles by genetic ablation of motor neurons ....................74

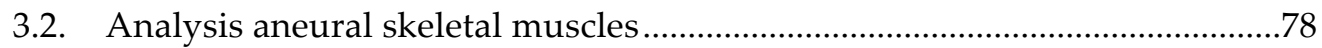


3.2.1. Removal of motor neurons results in skeletal muscle hypoplasia .........78

3.2.2. Abnormal muscle fiber morphology in $\mathrm{R} 26^{f x D T A} x \mathrm{Olig} 2^{\mathrm{Cre}}$ mice

3.2.3. Loss of motor neurons in mutants also leads to enhanced cell death in skeletal muscles .84

3.2.4. Slow myosin pattern is relatively preserved in aneural muscles. 86

3.2.5. Both primary and secondary myogenesis are affected by removal of motor neurons.

3.2.6. $R 26^{\text {fxDTA }} \mathrm{X} \mathrm{Olig} 2^{\mathrm{Cre}}$ mice display a compensatory increase in satellite cell numbers .92

3.2.7. Motor innervation is required for myofiber terminal differentiation

3.3. Strategy to block neurotransmission genetically

3.3.1. Using tetanus toxin light chain to eliminate neurotransmission in motor neurons.

3.3.2. TeNT protein is temporally expressed in mutant motor neurons throughout development.

3.3.3. Perturbing vesicular release in motor neurons results in skeletal muscle hypoplasia

3.3.4. Slow myosin expressing myofibers are generated in paralyzed muscles

3.3.5. Neurotransmission blockade does not abolish primary and secondary formation in $\mathrm{R} 26^{f x T e N T} x \mathrm{Olig} 2^{\mathrm{Cre}}$ muscles

3.3.6. Blocking neurotransmission results in an increase of motor neurons

3.3.7. Inactivating the Chat gene results in reduced muscle mass.

3.3.8. Blocked evoked neurotransmission did not disrupt sarcomere formation or organization.

3.4. Gene profiling of $\mathrm{R}_{2} 6^{f x D T A} x \mathrm{Olig} 2^{\mathrm{Cre}}$ aneural muscles. 
3.4.1. Differential gene expression underlying myogenesis in the absence of motor neurons

3.4.2. Potential myogenic effectors regulated by motor innervation 120

3.4.3. Muscle-associated genes affected by the removal of motor neurons .122

3.4.4. Embryonic denervation utilizes a set of molecules distinct from adult denervation pathway

3.4.5. Motor innervation regulates Ntf5 and Ntrk2 transcript levels in muscles

3.5. Gene expression profiles in paralyzed muscles of $R 26^{f x T e N T} x \mathrm{Olig} 2^{\mathrm{Cre}}$ mice...130

4. Discussion

4.1. Addressing the role of innervation in embryonic myogenesis

4.2. Primary and secondary myotubes form aneurally

4.3. Paradoxical hypertrophy of aneural muscle fibers 136

4.4. Innervation drives terminal differentiation of muscle fibers 138

4.5. Primary and secondary myotubes are formed during embryonic paralysis.

4.6. Different requirements of innervation for embryonic and postnatal muscles

4.7. Working model

5. Outlook

5.1. In ovo electroporation as a screening platform for innnervation-dependent myogenic candidate genes

5.2. Spinal motor neuron dependence on target muscles

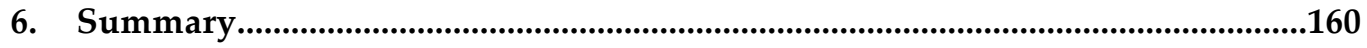

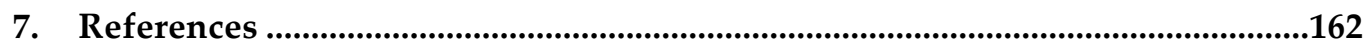

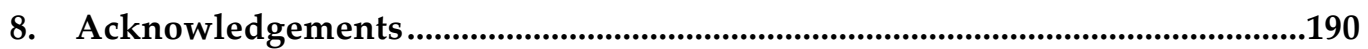

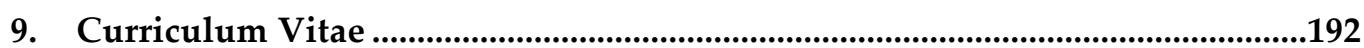




\section{List of figures}

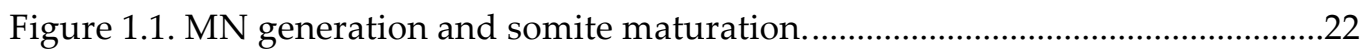

Figure 1.2. Spatial organization of motor neuron columns. ............................................24

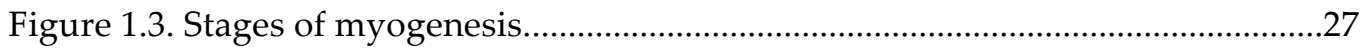

Figure 1.4. Three types of myoblasts that can potentially fuse to generate multinucleated myotubes.

Figure 1.5. Timeline of Pax3, Pax7 and MRFs expression during mouse embryogenesis.

Figure 1.6. The genetic hierarchy of limb myogenesis. .31

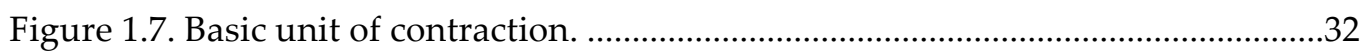

Figure 3.1: Strategy to genetically ablate motor neurons in mouse................................74

Figure 3.2: Motor neuron ablation does not affect somite delamination.......................75

Figure 3.3: Specificity of motor neuron ablation in mutant mouse lines.......................77

Figure 3.4: Removal of motor neurons results in general skeletal muscle hypoplasia.

Figure 3.5: Quantification of muscle loss in $\mathrm{R}^{66^{f x D T A}} x \mathrm{Olig} 2^{\mathrm{Cre}}$ and Isl2 ${ }^{\text {fxDTA }} x \mathrm{Olig} 2^{\mathrm{Cre}}$ mice.

Figure 3.6: Muscle fibers undergo hypertrophy after motor neuron ablation..............82

Figure 3.7: Abnormal myofiber morphology and organization in aneural mice. ........83

Figure 3.8: Aneural muscle fibers undergo progressive apoptosis.................................85

Figure 3.9: Slow myosin pattern was preserved in aneural muscles until E18.5.........87

Figure 3.10: Quantification of slow $\mathrm{MyHC}^{+}$fibers in $\mathrm{R}_{2} 6^{f x D T A} x \mathrm{Olig} 2^{\mathrm{Cre}}$ mice. ................88

Figure 3.11: Secondary myogenesis occurs in aneural muscles.....................................90

Figure 3.12: Compensatory increase in satellite cell numbers.......................................93

Figure 3.13: The motor nerve is required for the terminal differentiation of myotubes .95

Figure 3.14: Strategy to conditionally block neurotransmission in murine motor neurons. 
Figure 3.15: TeNT expression is specific to motor neurons. 100

Figure 3.16: Blocked neurotransmission leads to reduced muscle mass. 102

Figure 3.17: Apoptosis mediates reduced muscle mass in $R 26^{f x T e N T} x O \operatorname{lig} 2^{\text {Cre }}$ mice. ...103 Figure 3.18: Slow myosin pattern was preserved in paralyzed muscles until E18.5.

Figure 3.19: Secondary myogenesis occurs in paralyzed muscles. 106

Figure 3.20: Conditional expression of TeNT augments motor neuron survival......107

Figure 3.21: Blocking neurotransmission results in hyperinnervation of target muscles.

Figure 3.22: Deleting the Chat gene has severe impacts on myogenesis. 110

Figure 3.23: Striated muscle pattern is intact in paralyzed muscles 112

Figure 3.24: Downregulated gene profiles in $\mathrm{R} 26^{f \times D T A} x \mathrm{Olig} 2^{\mathrm{Cre}}$ muscles. 115

Figure 3.25: Heatmap of upregulated genes in $\mathrm{R} 26^{x \mathrm{xDTA}} x \mathrm{Olig} 2^{\mathrm{Cre}}$ muscles. 116

Figure 3.26: Biological function profiles of differentially expressed genes obtained via microarray.

Figure 3.27: Biological function profiles of differentially expressed genes obtained via RNA sequencing.

Figure 3.28: qPCR verification of downregulated candidate gene expression profiles throughout development.

Figure 3.29: qPCR verification of structural gene expression in aneural muscles throughout different developmental stages.

Figure 3.30: Embryonic denervation operates through a distinct pathway than adult.

Figure 3.31: qPCR verification of growth factors and receptors expression in aneural E15.5 muscles.

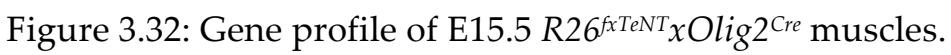
.131

Figure 4.1: Working hypothesis. 150

Figure 5.1: A screening platform for candidate gene function. 155

Figure 5.2: Strategy to study candidate gene function in context of denervation.....156

Figure 5.3: Different mouse lines to genetically abolish myogenesis. 158 


\section{List of tables}

Table 3.1: List of sarcomere-associated genes in control and

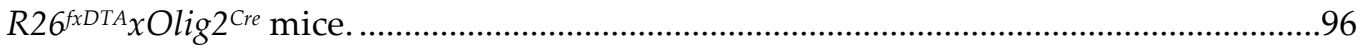

Table 3.2: List of sarcomere-associated genes in control and R26 fxTeNT $x$ Olig2 ${ }^{\text {Cre }}$ mice.

Table 3.3: DAVID analysis of top up- or downregulated canonical pathways.........119

Table 3.4: List of positively identified candidate genes in aneural muscles..............125

Table 3.5: List of downregulated structural genes in aneural muscles. ....................126 


\section{List of abbreviations}

\begin{tabular}{|c|c|}
\hline$\%$ & Percent \\
\hline${ }^{\circ} \mathrm{C}$ & Degree Celsius \\
\hline $\mathrm{ACh}$ & Acetylcholine \\
\hline $\mathrm{AChR}$ & Acetylcholine receptor \\
\hline ALD & Anterior latissimus dorsi \\
\hline Ankrd1 & Muscle ankryin repeat protein 1 \\
\hline Ankrd2 & Muscle ankryin repeat protein 2 \\
\hline Approx. & Approximately \\
\hline BDNF & Brain-derived neurotrophic factor \\
\hline bHLH & Basic helix-loop-helix \\
\hline BMP & Bone morphogenic protein \\
\hline bp & Base pair \\
\hline BR & Brachioradialis \\
\hline c-Met & MNNG HOS transforming gene \\
\hline $\mathrm{Ca}^{2+}$ & Calcium \\
\hline cDNA & Complementary DNA \\
\hline ChAT & Choline acetyltransferase \\
\hline Ckm & Muscle creatine kinase \\
\hline Cntf & Ciliary neurotrophic factor \\
\hline CSA & Cross-sectional area \\
\hline Cxcld & Chemokine ligand \\
\hline $\mathrm{D}$ & Dermomyotome \\
\hline D-tbc & D-Tubocurarine \\
\hline DAVID & $\begin{array}{l}\text { Database for Annotation, Visualization and } \\
\text { Integrated Discovery }\end{array}$ \\
\hline DCM & Dilated cardiomyopathy \\
\hline
\end{tabular}




\begin{tabular}{ll} 
DML & Dorsomedial lip of dermomyotome \\
DNA & Deoxyribonucleic acid \\
dpc & Day of post-conception \\
DRG & Dorsal root ganglion \\
DTA & Diphtheria toxin subunit A \\
E & Embryonic day \\
EAG & Ether-a-go-go \\
ECR & Extensor carpi radialis brevis \\
EDC & Extensor digitorum commusis \\
EDL & Extensor digitorum longus \\
EdU & 5-Ethynyl-2'-deoxyuridine \\
Eg & Exempli gratia \\
eGFP & Enhanced green fluorescent protein \\
Eno3 & Enolase 3, beta muscle \\
EPP & End plate potentials \\
Esrrb & Estrogen related receptor beta \\
Etc & Forkhead box protein muscle isoform \\
ETS & Fibroblast growth factor muscle isoform \\
Etv4 & E-twenty six \\
EYA & ETS translocation variant 4 \\
F & Fyes absent homologue \\
f & Flexor carpi radialis \\
FCR & Flexor carpi ulnaris \\
FCU & FDP \\
FGF & Fible group \\
\hline
\end{tabular}




\begin{tabular}{ll} 
g & Grams \\
Gdnf & Glyceraldehyde-3-phosphate 7l \\
GFP & Glial derived neurotrophic factor \\
Gl & Green fluorescent protein \\
Gm & Lateral gastrocnemius \\
Gsk3 $\beta$ & Medial gastrocnemius \\
h & Glycogen kinase 3 $\beta$ \\
HCM & Hour \\
Hdac & Hypertrophic cardiomyopathy \\
Hgf/Sf & Histone deacetylase \\
HH & Hepatocyte growth factor/Scatter factor \\
Hhd & Hamburger-Hamilton \\
HMC & Hedgehog family muscle isoform \\
IGF1-Akt/PKB & Hypaxial motor column \\
IN & Insulin-like growth factor 1-Akt/protein kinase B \\
Isl1/2 & Interneuron \\
KEGG & Islet 1/2 \\
Kir2.1 & Kyoto Encyclopedia of Genes and Genomes \\
l & Inward rectifier potassium channel \\
LMC & Liter \\
loxP & Lateral motor column \\
M & Floxed \\
M & Molar \\
MAFbx & Myotome \\
Marp & Muscle Atrophy F-box \\
Min & Munkyrin repeat protein \\
\hline
\end{tabular}




\begin{tabular}{|c|c|}
\hline MLC & Myosin light chain 1F/3F \\
\hline MLCK & Myosin light chain kinase \\
\hline $\mathrm{mm}$ & Milli \\
\hline MMC & Medial motor column \\
\hline $\mathrm{MN}$ & Motor neuron \\
\hline MRF & Myogenic regulatory factor \\
\hline mRNA & Messenger ribonucleic acid \\
\hline mTOR & Mammalian target of rapamycin \\
\hline MuRF1 & Muscle RING Finger 1 \\
\hline Myfd & Myogenic regulatory factor for terminal differentiation \\
\hline MyHC & Myosin \\
\hline MyHC-emb/Myh3 & Myosin heavy chain, embryonic isoform \\
\hline MyHC-fast & Myosin heavy chain, fast isoform \\
\hline MyHC-neo/Myh8 & Myosin heavy chain, neonatal isoform \\
\hline MyHC-slow/Myh7 & Myosin heavy chain, slow isoform \\
\hline Myl2 & Myosin light chain, cardiac slow isoform \\
\hline Myl3 & Myosin light chain, skeletal slow isoform \\
\hline MyoG & Myogenin \\
\hline $\mathrm{N}$ & Notochord \\
\hline Nfat & Nuclear factor of activated T-cells \\
\hline $\operatorname{Ngf}$ & Nerve growth factor \\
\hline $\mathrm{NMJ}$ & Neuromuscular junction \\
\hline Ntf3 & Neurotrophin 3 \\
\hline Ntf5 & Neurotrophin $4 / 5$ \\
\hline Ntrk2 & Neurotrophic tyrosine kinase, receptor, type 2 \\
\hline $\mathrm{P}$ & Peroneus muscle group \\
\hline PBS & Phosphate buffered saline \\
\hline pCAGGS & Chick beta-actin promoter \\
\hline PCD & Programmed cell death \\
\hline
\end{tabular}




\begin{tabular}{|c|c|}
\hline PCR & Polymerase chain reaction \\
\hline PFA & Paraformaldehyde \\
\hline PGC & Preganglionic chain \\
\hline Pkctd & Protein kinase $\mathrm{C}$ muscle isoform \\
\hline PL & Pollicis longus \\
\hline PLD & Posterior lattisimus dorsi \\
\hline PMA & Peroneal muscular atrophy \\
\hline pMN & Motor neurons progenitor domain \\
\hline PT & Pronator teres \\
\hline qPCR & Quantitative polymerase chain reaction \\
\hline $\mathrm{r}$ & Radial bone \\
\hline RA & Retinoic acid \\
\hline RNA & Ribonuclei acid \\
\hline rpm & Revolutions per minute \\
\hline$S$ & Sclerotome \\
\hline S6K & S6 kinase \\
\hline Sapd & Sarcomere and calcineurin associated protein \\
\hline SEM & Standard error of the mean \\
\hline Shh & Sonic hedgehog \\
\hline Six & Sine oculis homeobox \\
\hline Snaid & Snail homologue muscle isoform \\
\hline SNAREs & $\begin{array}{l}\text { Soluble N-ethylmaleimide-sensitive factor attachment } \\
\text { protein receptor }\end{array}$ \\
\hline $\mathrm{t}$ & Tibial bone \\
\hline TeNT & Tetanus toxin light chain \\
\hline TeTx & Tetanus toxin \\
\hline tPA & Stop sequence \\
\hline TTX & Tetrodotoxin \\
\hline Tuba1b & Tubulin, alpha $1 b$ \\
\hline TUNEL & Terminal deoxynucleotidyl transferase dUTP nick end \\
\hline
\end{tabular}


$\mathrm{u}$

vAChT

VAMP2

$\mathrm{VH}$

VLL

$\mathrm{w} / \mathrm{v}$

$\alpha$-BTX

$\beta$-BTX

$\mu$
Ulnar bone

Vesicular acetylcholine transporter

Synaptobrevin

Ventral horn

Ventrolateral dermomyotome

Weight per volume

Alpha-Bungarotoxin

Beta-Bungarotoxin

Micro 


\section{Introduction}

"In science it is a service of the highest merit to seek out those fragmentary truths attained by the ancients, and to develop them further." Johann Wolfgang von Goethe

\subsection{The neuromuscular system}

Higher animal life is defined by the ability to perform controlled movement set forth via the activation of skeletal muscle by motor neurons through neuromuscular junctions (NMJ). The vertebrate NMJ comprises three principle cell types: the presynaptic motor neuron (spinal or lower motor neurons), the postsynaptic muscle fiber and the Schwann cell (Sanes and Lichtman, 1999). NMJs transmit electrical signals from motor neurons located in the ventral part of the spinal cord to the target muscles. These electrical impulses (termed action potentials) are propagated along the length of the motor axon where, upon arrival at the presynaptic motor axon terminal, cause an activation of voltage-gated calcium ion channels ( $\mathrm{P} / \mathrm{Q}$ types) leading to an influx of extracellular $\mathrm{Ca}^{2+}$, finally resulting in the exocytosis of synaptic vesicles containing the neurotransmitter Acetylcholine (ACh). ACh, which serves as the main neurotransmitter in the neuromuscular system, is released into the synaptic cleft where it binds to its receptors located on the postsynaptic muscle fiber membrane. Activation of these muscle-type nicotinic ACh receptors (AChRs) leads to the depolarization of the muscle fibers and eventually contraction, the basis for movement of any kind to occur.

During mouse early development, muscle-type nicotinic AChRs clusters are prepatterned on muscle fibers at around E12.5-E13.5 in the diaphragm (Creazzo and Sohal, 1983) independently from motor innervation (Lin et al., 2001), and they are initially composed of $\alpha_{2} \beta \gamma \delta$ subunits (embryonic-type). Subsequently, a conversion to $\alpha_{2} \beta \varepsilon \delta$ subunits (adult-type) takes place at the NMJ between later embryonic 
development and early postnatal period to allow for optimal innervation of muscle fibers (Yampolsky et al., 2008).

The interaction between motor neurons, skeletal muscles and glial cells is fundamental to NMJ formation. Factors released from motor neurons have been shown to control postsynaptic differentiation directly by stimulating receptors on muscle cells or indirectly by promoting glial cell differentiation and function (Wu et al., 2010). The tight-knitted relationship between motor neurons and muscles is such that one is unable to function in the absence of the other, which is exemplified by neuromuscular disorders, such as neuropathies. Due to its accessibility, the neuromuscular system is a classical model for tissue-interaction and synaptogenesis (Lei et al., 2012). Even though a plethora of classical embryological and physiological studies have investigated the interaction between motor neurons and muscle fibers during development, the precise roles these interactions play in the formation of the neuromuscular system have long been controversial, while the underlying mechanisms remain poorly resolved.

\subsection{Motor neurons}

The assembly of spinal cord circuits that drives movements requires the generation of diverse cell types (Dasen and Jessell, 2009). Work over the past two decades has begun to shed light on the molecular programs that function during embryonic development to determine motor neuron differentiation and motor neuron-muscle connectivity (Dasen and Jessell, 2009; Bonanomi and Pfaff, 2010). The control of various motor behaviors demands the orchestrated activation of different muscles, each by a committed set of motor neurons (Dasen and Jessell, 2009). Hence, to supplely adapt in response to biomechanical challenges posed by the environment, different types of motor neurons are generated which can be categorized according to their developmental transcriptional codes, topographic position within the spinal cord and 
finally, their physiological functions (Dasen and Jessell, 2009; Manuel and Zytnicki, 2011).

\subsubsection{Motor neuron development}

During spinal cord development, spinal motor neurons and several classes of interneurons are generated from neural progenitor cells upon exposure to graded extrinsic signals along the dorsoventral axis of the neural tube (Dasen and Jessell, 2009). These secreted factors comprising sonic hedgehog (Shh), fibroblast growth factors (FGFs) and retinoic acid (RA) initiate distinct combinatorial expression domains of transcription factors in neural progenitors, thereby defining progenitor fates (Briscoe et al., 2000; Jessell, 2000; Shirasaki and Pfaff, 2002; Dasen and Jessell, 2009). A ventral-to-dorsal gradient of Shh is the main signaling pathway responsible for the generation of the most ventral cell types, including motor neurons, whereas a dorsal-to-ventral gradient of bone morphogenic proteins (BMP) is responsible for the generation of dorsal interneurons (Jessell, 2000). Secretion of Shh by the notochord and floor plate generates a ventral-to-dorsal gradient, giving rise to six progenitor cell domains within the ventral neural tube (Figure 1.1) (Briscoe et al., 2000). Homeodomain proteins expressed by the progenitor cells have been suggested to be involved in identity specification in the classes of postmitotic cells that arise from each progenitor domain. Motor neurons arise from one of the progenitor domains termed motor neurons progenitor ( $\mathrm{pMN}$ ) domain situated in the ventricular layer and they migrate laterally to the marginal layer of the prospective ventral horn (Jansen and Fladby, 1990). In response to Shh, the successful acquisition of motor neuron cell fate is determined by the spatiotemporal expression of a unique profile of transcription factors, the homeodomain proteins Nkx6.1, Nkx6.2, Pax6 and the activities of basic helix-loop-helix (bHLH) protein Olig2 in the pMN domain, (Briscoe et al., 2000; Cai et al., 2000; Vallstedt et al., 2001). Gain-of-function studies have shown Olig2 to be 
capable of inducing ectopic motor neurons generation (Marquardt and Pfaff, 2001; Novitch et al., 2001), which involves induction of the expression of Neurogenin 2 which is thought to act as a generic proneural factor to promote cell cycle exit, suppress Notch signaling and glial fate, while factors like $\mathrm{Hb} 9$ and Isl1 drive motor neuron-specific programs (Lee et al., 2005). The expression of Neurogenin 2 in the pMN domain is downregulated after the initial phase of motor neuron generation, which eventually switches to generate oligodendrocytes instead; a process thought to be initiated by a ventral-to-dorsal shift in Shh gradient (Richardson et al., 2000; Marquardt and Pfaff, 2001; Novitch et al., 2001; Takebayashi et al., 2002; Lee et al., 2005).

Postmitotic spinal motor neurons express a set of homeodomain transcription factors (notably $\mathrm{Hb} 9$ and Isl2) that regulate common spinal motor neuron features (Pfaff et al., 1996; Arber et al., 1999; Thaler et al., 1999; Sharma et al., 2000), while additional factors, including Hox family transcription factors, promote further motor neuron subtype diversification (Dasen and Jessell, 2009). The earliest postmitotic motor neurons appear at embryonic day (E) 9.5 in the mouse spinal cord (Dasen and Jessell, 2009), which coincides with the extension of their axons out of the ventral spinal cord following a defined trajectory, such as the limb or trunk mesenchyme. The latest postmitotic motor neurons are generated at E12.5, followed by the initiation of oligodendrogenesis.

Movements of terrestrial vertebrates are mediated by hundreds of anatomically distinct muscle groups (Dasen and Jessell, 2009) that serve different functions. The motor neurons that innervate these peripheral targets are organized into discrete clusters that occupy distinct domains along the rostrocaudal axis of the spinal cord, and are termed motor columns (Jessell, 2000). The topographic location of motor neurons in each column is often predictive of target innervation pattern that link their cell bodies to their synaptic targets located in the periphery (Hollyday and Jacobson, 
1990; Dasen and Jessell, 2009; Bonanomi and Pfaff, 2010).

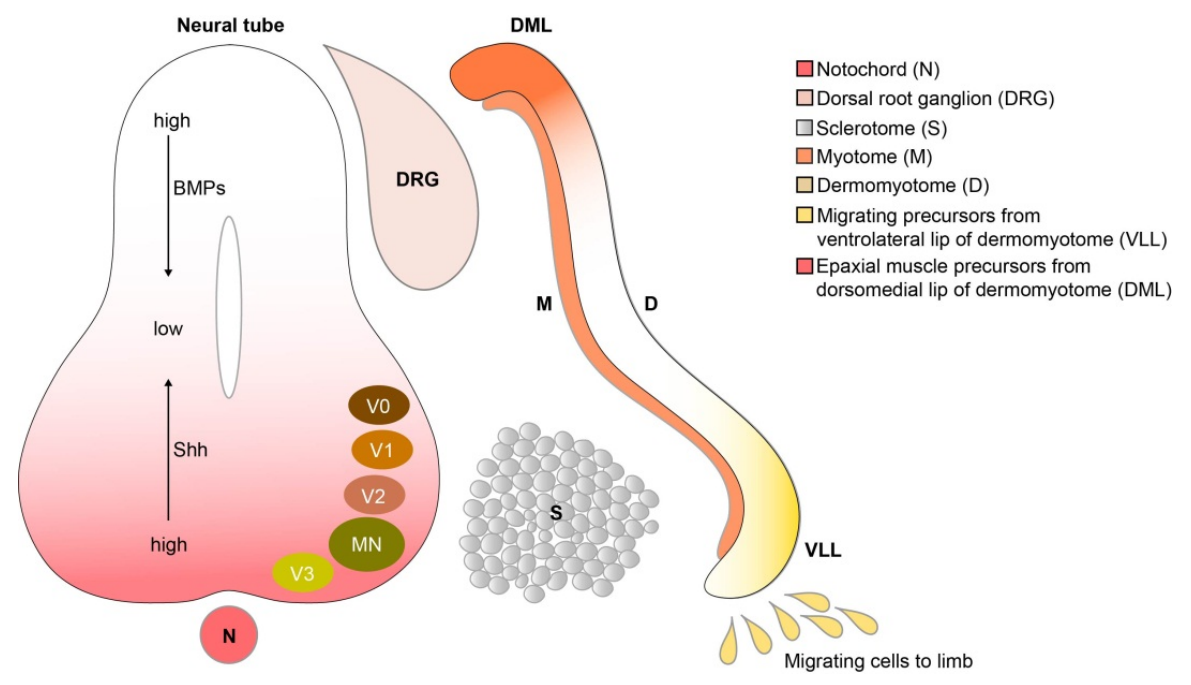

Figure 1.1. MN generation and somite maturation.

Motor neurons and ventral interneurons are generated in progenitor domains $\mathrm{pMN}$ and pV0-V3, respectively, according to a gradient of sonic hedgehog (Shh) derived from the floor plate and notochord. Dorsal interneurons are generated via bone morphogenic proteins (BMPs) gradient derived from the roof plate. Paraxial mesoderm condensed into somites, which delaminate to give rise to three distinct structures. The sclerotome (S) generates future ribs and vertebrae, whereas migratory muscle limb precursors are derived from the VLL of dermomyotome. The central dermomyotome gives rise to epithelial, dorsal dermis and smooth muscles. The underlying myotome derived from the DML of dermomyotome is a source of back and abdominal muscles.

Motor neurons having similar motor axon trajectories, e.g. projecting into the limbs or trunk muscles, extend their peripheral axons together. Additional levels of organization are present within the defined motor columns, as seen by the segregation of motor columns into "divisions" and "pools" (Dasen and Jessell, 2009). At both the brachial and lumbar level of the spinal cord, the lateral motor column (LMC) is divided into two divisions (Figure 1.2): the medial division ( $\mathrm{LMCm}$ ) contains neurons which project ventrally and the lateral division $(\mathrm{LMCl})$ which contains neurons that project dorsally within the limb mesenchyme (Landmesser, 1978; Tosney and Landmesser, 1985a; Tosney and Landmesser, 1985c). The axons of the LMCm with 
earlier birth dates will innervate future extensor muscles whereas axons of the LMCl will innervate the future flexor muscles of the limb (Jansen and Fladby, 1990).

\subsubsection{Motor neuron classes}

Since being discovered in the late 19th century by Sir Charles Scott Sherrington, the knowledge about motor neurons has been interminably advancing. As of now, the 21st century, it is known that motor neurons make up a very heterogeneous population of neurons; they innervate different muscle fiber types, have different intrinsic electrical and molecular properties, morphology and exhibit different degrees of vulnerability to degeneration (Manuel and Zytnicki, 2011). Briefly, motor neurons can be classified into alpha $(\alpha)$-, beta $(\beta)$ - and gamma $(\gamma)$-motor neurons. $\alpha$-motor neurons innervate extrafusal skeletal muscle fibers, causing their contraction hence allowing movement. They are the most abundant within the classes with a ratio of 2:1 to $\gamma$-motor neurons (Hunt and Kuffler, 1951; Friese et al., 2009), and besides their intrinsic properties, they can be subdivided by the contractile properties of the muscle fiber type (type I, IIa, IIb) that they innervate. A single $\alpha$-motor neuron innervates a subset of muscle fibers with the same contractile properties within a muscle, which constitutes a motor unit. $\gamma$-motor neurons innervate intrafusal fibers in the muscle spindle, receive proprioceptive feedback from muscle spindles in the muscles and participate in regulating muscle tone and length by modulating the stretch sensitivity of muscle spindles (Hunt and Kuffler, 1951). $\beta$-motor neurons, on the other hand, are a less characterized group. They are thought to innervate both extra- and intrafusal fibers but their exact functions remain obscure. 


\subsubsection{Organization of motor neurons within the spinal cord}

Movements of terrestrial vertebrates are mediated by hundreds of anatomically distinct muscle groups (Dasen and Jessell, 2009) that serve different functions. The motor neurons that innervate these peripheral targets are organized into discrete clusters that occupy distinct domains along the rostrocaudal axis of the spinal cord, and are termed motor columns (Jessell, 2000). The topographic location of motor neurons in each column is often predictive of target innervation pattern that link their cell bodies to their synaptic targets located in the periphery (Hollyday and Jacobson, 1990; Dasen and Jessell, 2009; Bonanomi and Pfaff, 2010).

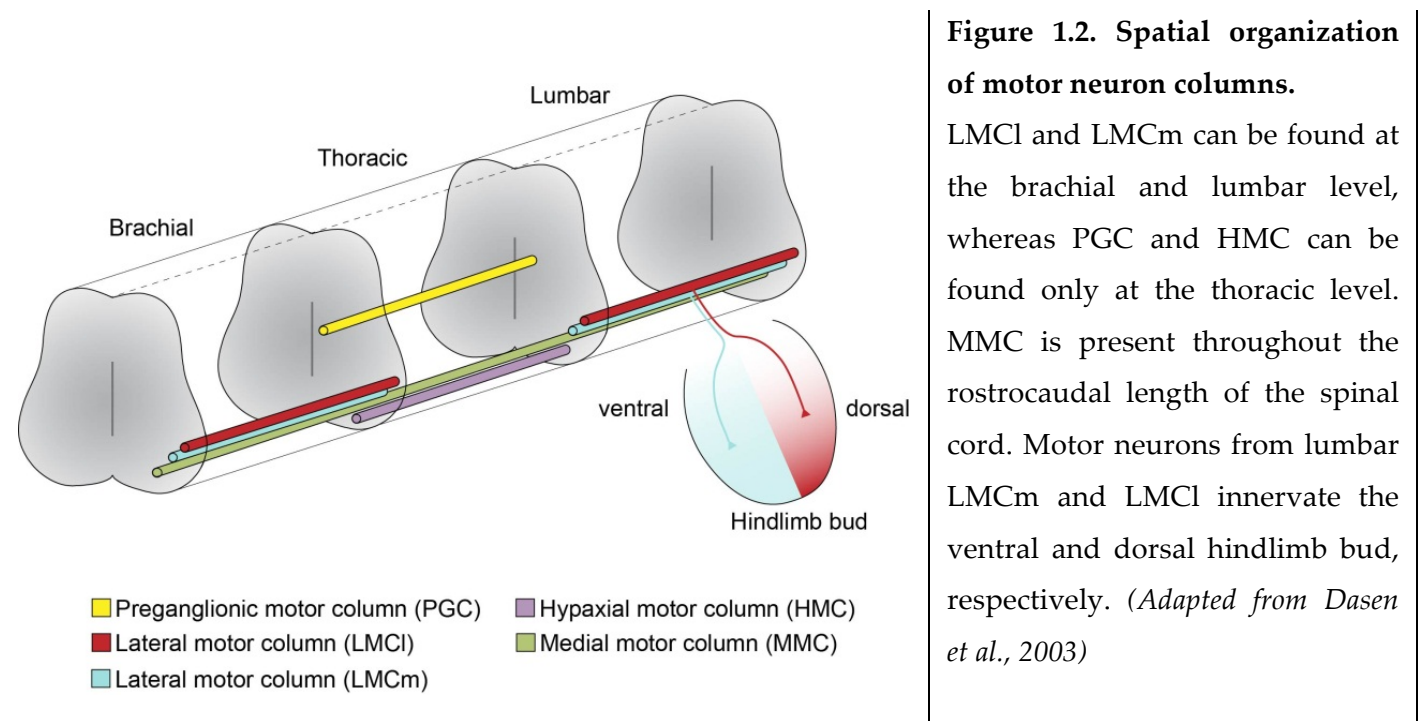

Motor neurons having similar motor axon trajectories, e.g. projecting into the limbs or trunk muscles, extend their peripheral axons together. Additional levels of organization are present within the defined motor columns, as seen by the segregation of motor columns into "divisions" and "pools" (Dasen and Jessell, 2009). At both the brachial and lumbar level of the spinal cord, the lateral motor column (LMC) is divided into two divisions (Figure 1.2): the medial division (LMCm) contains neurons which project ventrally and the lateral division $(\mathrm{LMCl})$ which contains neurons that 
project dorsally within the limb mesenchyme (Landmesser, 1978; Tosney and Landmesser, 1985a; Tosney and Landmesser, 1985c). The axons of the LMCm with earlier birth dates will innervate future extensor muscles whereas axons of the LMCl will innervate the future flexor muscles of the limb (Jansen and Fladby, 1990).

\subsection{Generation of skeletal musculature in vertebrates}

Muscles are contractile organs, utilizing myosin II motor proteins and actin filaments to generate force and movement. In vertebrates, there are three types of muscles: smooth, cardiac and skeletal. Smooth muscle is found in visceral organs and vasculature, cardiac muscle is used by the heart for pumping blood, whereas skeletal muscles are responsible for body movements and locomotion. Of these, cardiac and skeletal muscles have a striated appearance arising from the highly ordered arrangement of actin and myosin filaments (Braun and Gautel, 2011). The vertebrate skeletal musculature consists of functionally distinct components generated during embryogenesis (Bryson-Richardson and Currie, 2008).

\subsubsection{Delamination and migration of muscle progenitor cells}

During embryonic development, skeletal muscles are derived from the paraxial mesoderm, which later condenses to develop into spherical epithelial structures termed somites. A total of 65 pairs of somites are formed in the mouse embryo (Tam, 1981). Somites are formed in a rostrocaudal sequence, and generate three distinct lineage-restricted cellular compartments under inductive influence of neighboring tissues: the sclerotome, myotome and dermomyotome (Figure 1.1) (Christ et al., 1992; McMahon et al., 1998). The ventral epithelial-derived sclerotome gives rise to the future vertebrae and ribs (Christ and Ordahl, 1995), while the central dermomyotome de-epithelializes and becomes the source of precursors of the dorsal dermis, 
endothelial and smooth muscles. The myotome is comprised of an epaxial and a hypaxial component. The epaxial myotome is formed by cells that have delaminated from the dorsomedial lip of the dermomyotome and gives rise to deep back muscles, while the hypaxial myotome contains cells that have delaminated from the ventrolateral lip of the dermomyotome and contributes to abdominal muscles at thoracic levels (Tajbakhsh and Buckingham, 2000). At limb bud levels, the cells located at the ventrolateral lip of the dermomyotome will migrate to invade the lateral plate mesoderm via positional cues provided by the mesenchymal cells of the limbs where they eventually differentiate into skeletal muscles (Christ and Ordahl, 1995; Brent and Tabin, 2002; Buckingham et al., 2003). This migration involves critical molecules like hepatocyte growth factor/scatter factor $(\mathrm{Hgf} / \mathrm{Sf})$ and its receptor c-Met (Dietrich et al., 1999; Bentzinger et al., 2012). In the mouse, it is initiated around E9.25 in the forelimb and is completed by E11.0 in the hindlimb (Bismuth and Relaix, 2010). Genetic inactivation of either $\mathrm{Hgf} / \mathrm{Sf}$ or c-Met results in mice devoid of skeletal limb muscles (Bladt et al., 1995; Schmidt et al., 1995).

\subsubsection{Cellular events during myogenesis}

The migrating precursor cells from the ventrolateral lip of the dermomyotome invade the limb bud as myogenesis - the commitment of precursor cells to the muscle lineage - begins (Buckingham et al., 2003). This is a process tightly regulated by a network of transcription factors leading eventually to the expression of myogenic determination genes and differentiation of contractile muscle fibers.

Three types of muscle cell precursors (termed myoblasts) arise during embryonic mouse development, initiating two waves of myogenesis (Kelly and Zacks, 1969) (Ontell and Kozeka, 1984; Ross et al., 1987). Embryonic myogenesis occurs in newly formed somites whereby the dorsal progenitors respond to inductive signals like Shh and Wnts produced by the neural tube, notochord and floor plate to activate the 
expression of Myf5 and MyoD that commit cells to myogenesis (Cossu and Borello, 1999; Pownall et al., 2002). For example, mice deficient for Shh have reduced Myf5 expression in addition to lacking epaxial musculature (Borycki et al., 1999). In the mouse embryonic limb, primary myogenesis begins at $\sim$ E11.5 and ends at $\sim$ E14.5, generating multinucleated structures called primary myotubes resulting from the endto-end fusion of primary (embryonic) myoblasts derived from $\mathrm{Pax}^{+}$progenitor cells (Figure 1.3) (Zhang and McLennan, 1995; Buckingham et al., 2003; Hutcheson et al., 2009). The appearance of the first myotubes in the limb bud arising at E12.5 coincides with the arrival of the ventrally and dorsally projecting LMC motor axons to the ventral and dorsal muscle mass, respectively (Tosney and Landmesser, 1985c). These muscle masses will eventually undergo segregation to form distinct muscles.

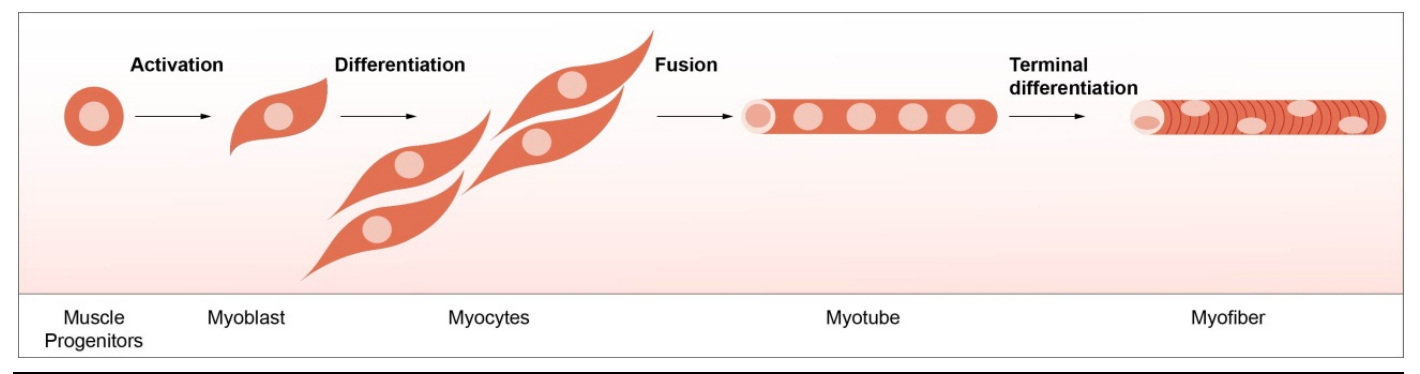

Figure 1.3. Stages of myogenesis. Muscle progenitors are activated to form myoblasts, which have proliferative capability and are committed to a myogenic fate. Myoblasts undergoing differentiation exit the cell cycle and elongate to form myocytes. The myocytes align and fuse end-to-end to give rise to multinucleated myotubes, the precursors of mature myofibers. During terminal differentiation, myotubes mature and form contractile apparatus necessary for functional contraction.

Fusion of myoblasts into multinucleate syncytia is a tightly controlled and fundamental step in muscle differentiation, which involves cell migration, adhesion and fusion (Abmayr and Pavlath, 2012). The fusion process is promoted by a change in membrane potential, which requires the synchronized activity of three types of ion channels, notably inward rectifier $\mathrm{K}^{+}(\mathrm{Kir} 2.1)$, ether-a-go-go (EAG) and T-type $\mathrm{Ca}^{2+}$ channels. After primary myogenesis, secondary myogenesis commences (Bernheim et 
al., 1996; Fischer-Lougheed et al., 2001). This entails the fusion of fetal myoblasts which are cells that develop from distinct, but related (Pax3 -derived Pax $7^{+}$) progenitors along the length of the existing primary myotubes (Figure 1.4) (Duxson et al., 1989).

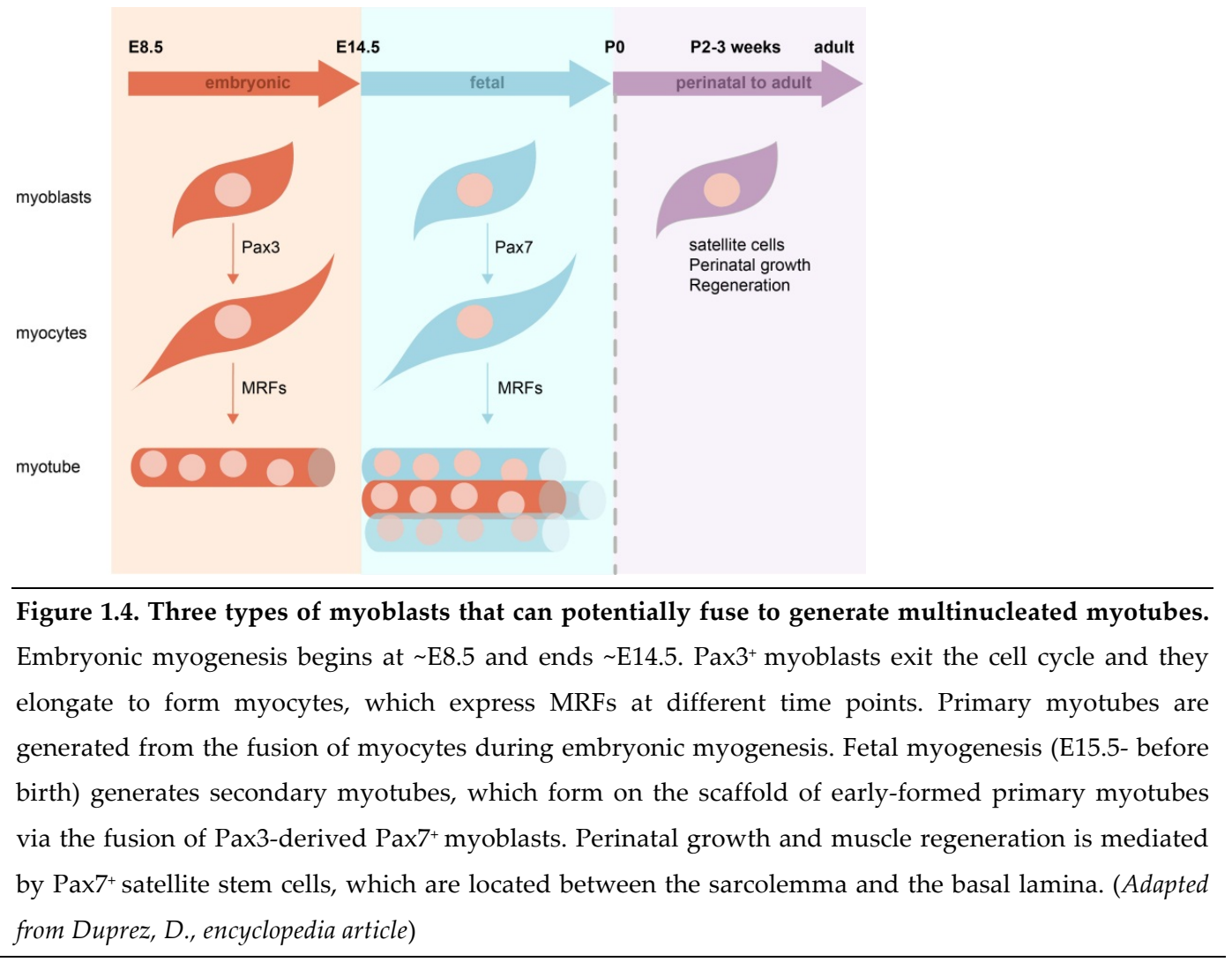

The secondary fibers are encapsulated by the same basal lamina as the primary fibers on which they are formed and where they elongate to eventually become independent fibers. Secondary fibers can be distinguished from primary fibers by their relative small size (Kelly and Zacks, 1969). Later in development, from E16.5 onwards, a third type of Pax $7^{+}$myoblasts known as satellite cells can be morphologically identified between the basal lamina and the sarcolemma. These cells become solely responsible for postnatal muscle growth and regeneration (Figure 1.4) (Sambasivan and 
Tajbakhsh, 2007; Lepper et al., 2011; Murphy et al., 2011).

\subsubsection{Molecular events during myogenesis}

Generation and terminal differentiation of skeletal muscle is controlled by members of myogenic regulatory factors (MRFs) (Braun and Gautel, 2011). The MRFs belong to the basic helix loop helix (bHLH) family of proteins. The basic domain of bHLH proteins mediates DNA binding, while the helix-loop-helix segment forms heterodimers with E proteins. MRF-E protein complexes bind to E-box motifs located upstream of many muscle-specific genes (Massari and Murre, 2000). MRFs act together with other transcriptional cofactors to drive the expression of genes required for the skeletal muscle developmental program (Kablar and Rudnicki, 2000). There are four members in this family, comprising myogenic factor 5 (Myf5), myogenic differentiation 1 (Myod1/MyoD), muscle-specific regulatory factor 4 (Mrf4 or Myf6) and myogenin (Bryson-Richardson and Currie, 2008; Braun and Gautel, 2011). While ectopically expressing any of the factors is sufficient to confer a myogenic fate in nonmuscle cells, the MRFs each display distinct temporospatial expression patterns and seem to perform different roles in the myogenic lineage (Figure 1.5) (Rawls et al., 1995). Myod1 and Myf5 are thought to play roles in muscle cell determination upstream of the other MRFs (Berkes and Tapscott, 2005). Determination genes like MyoD and Myf5 are implicated in the maintenance of muscle progenitor lineages and are considered markers of terminal commitment to the muscle lineage (Pownall et al., 2002). However, although they can initiate certain gene regulatory processes of myogenic specification (i.e. expression of early muscle differentiation markers, etc.), their actions alone are insufficient to drive the regulatory program for muscle differentiation during later development, underscoring the requirement for other MRFs. Myogenin appears to be crucial for the terminal differentiation of committed myoblasts, whereas Myf6 has a dual role in being able to act both as a differentiation gene in postmitotic 
cells and as a determination gene in proliferating myoblasts (Kassar-Duchossoy et al., 2004).

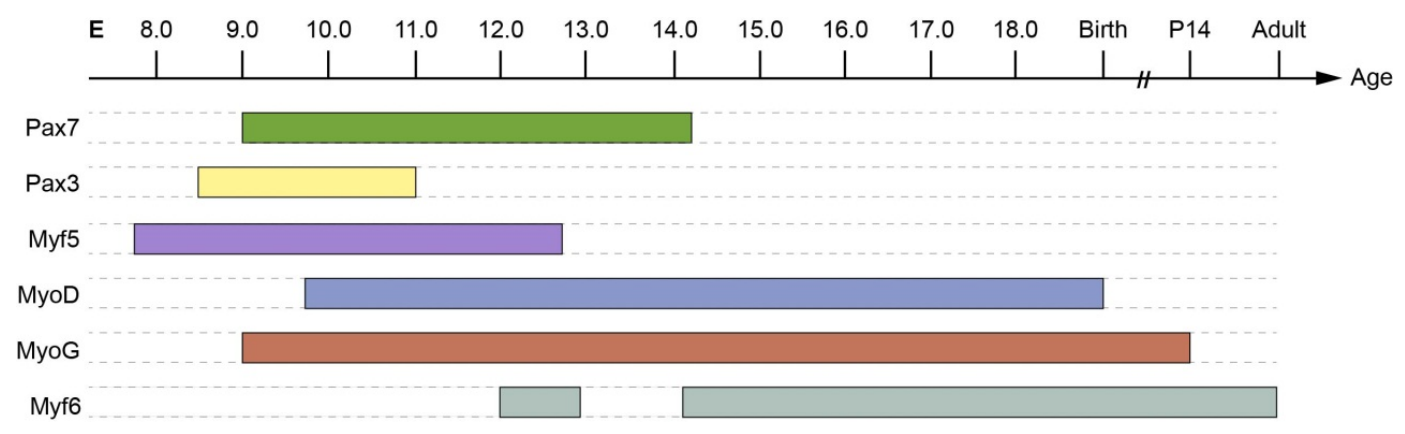

Figure 1.5. Timeline of Pax3, Pax7 and MRFs expression during mouse embryogenesis. Colored boxes indicate timed expression of Pax7 (green), Pax3 (yellow), Myf5 (purple), MyoD (blue), myogenin (MyoG) (brown) and Myf6 (light green) in the somite and limbs. Pax7, Pax3 and Myf5 are transiently expressed, whereas MyoD, MyoG and Myf6 are expressed throughout development. Myf6 has a biphasic expression, appearing at E12.0-E13.0 and reappearing at around E14.0 in the limb again. It is the most highly expressed MRF in postnatal muscles. (Adapted from Eftimie et al., 1991; Borycki and Emerson, 1997)

Gene targeting in mice has shed light on the role of MRFs during myogenesis. Null mutations in myogenin almost completely abolishes skeletal muscle differentiation; mutant myogenin mice have severe reduction in skeletal muscle mass, resulting from diffuse myofiber formation and an abundance of undifferentiated myoblasts (Hasty et al., 1993; Nabeshima et al., 1993; Ordahl and Williams, 1998). Contrastingly, mutations in either Myf5, MyoD or Myf6 do not alter the patterning and amount of skeletal muscle tissue (Braun et al., 1992; Rudnicki et al., 1992; Zhang et al., 1995). Inactivating either Myf5 or MyoD genes, however, causes delayed onset of differentiation of the epithelial-derived musculature (back, intercostals and abdominal wall), as well as the hypaxial-derived musculature (branchial arches, tongue, limbs, diaphragm, intercostals and abdominal wall), respectively (Kablar et al., 1997; Kablar et al., 1998). Simultaneous inactivation of Myf5 and MyoD results in amyogenic embryos lacking both differentiated muscle and myoblasts (Rudnicki et al., 1992). 


\subsubsection{Genetic hierarchy implicated in limb myogenesis}

According to the genetic hierarchy implicated during myogenesis (Figure 1.6), these MRF genes act downstream of or in parallel with homeodomain-and paired containing transcription factors Pax3 and Pax7 in different phases of embryonic and adult myogenesis (Bryson-Richardson and Currie, 2008). Pax3, is itself regulated by upstream factors that coordinate lineage specification, such as sine oculis homeobox protein 1 and 4 (Six 1 and Six4) as well as their cofactors eyes absent homologue 1 and 2 (Eya1 and Eya2) (Figure 1.6).

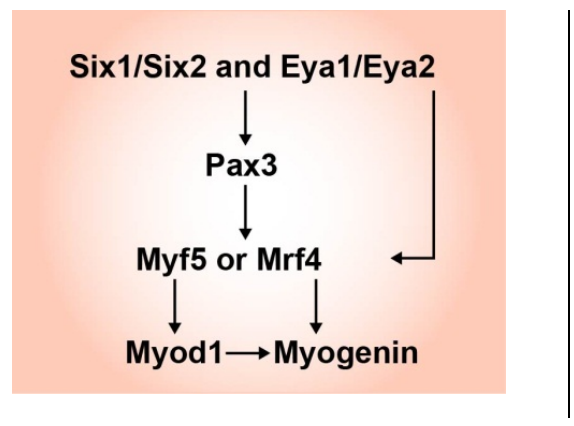

Figure 1.6. The genetic hierarchy of limb myogenesis. Muscles from the limbs are derived from trunk somites and expression of all MRFs is essential for driving myogenic differentiation. Upstream, Six and Eya proteins regulate Pax3, which controls the proliferative myogenic precursor pool. (Adapted from (Braun and Gautel, 2011)

Pax7, on the other hand, is dispensable for early myogenesis (Tajbakhsh et al., 1997), but is critical for the specification and maintenance of satellite-cell populations responsible for postnatal muscle growth and regeneration (Seale et al., 2000; Relaix et al., 2005). Inductive signals (for e.g., Wnts, Shh, BMPs, Notch-Delta1) from axial structures also act to regulate expression of MRFs (Bentzinger et al., 2012). Aside from MRF members, other transcription factors, such as the Mef2 family, which contain MADS-box-motifs, act in concert with the other myogenic factors to promote myoblast differentiation (Black and Olson, 1998; Buckingham et al., 2003) 


\subsubsection{Organization of muscles}

Two major classifications can be used to distinguish vertebrate muscles: the cardiac and skeletal muscles which are collectively termed as striated muscles, as opposed to the smooth muscles. Assembly of discrete units termed sarcomeres into myofibrils accounts for the striated appearance of the muscles. A sarcomere, the basic contractile unit of the muscle, is composed of antiparallel actin and myosin filaments, titin filaments as well as crosslinking proteins like $\alpha$-actinin, desmin and myomesin (Figure 1.7). Additionally, the sarcomere contains accessory proteins found at the Z-disk and/or the I-band to control transcription and protein turnover (Braun and Gautel, 2011).

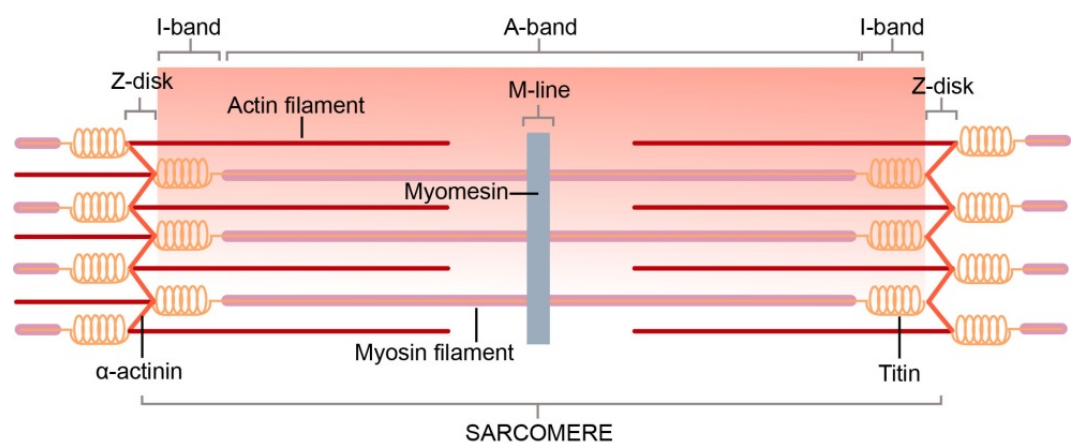

Figure 1.7. Basic unit of contraction. A sarcomere is the basic contractile unit of the cardiac and skeletal muscles. The core sarcomere consists of antiparallel actin and myosin filaments, titin filaments and crosslinker proteins for actin such as myosin, myomesin and $\alpha$-actinin. In addition, there are accessory constituents like proteins involved in transcriptional regulation and turnover control. (Adapted from Braun and Gautel, 2011)

Myofibrillogenesis is initiated at E9 when cells in the myotome domain of cervical somites begin to express MRF proteins (Furst et al., 1989). Shortly after, the sequential expression of muscle-specific proteins can be detected: desmin first, followed by titin, muscle-specific actin and myosin heavy chain proteins and finally nebulin (Furst et al., 1989). However, it is only after fusion of myoblasts to myotubes beginning at $\sim$ E13 in the mouse that the characteristic myofibrillar banding pattern can be observed (Furst 
et al., 1989). Myofibril assembly starts after myotube fusion with the formation of myofibrils at the periphery of the newly fused myotube (Myhre and Pilgrim, 2012). The myofibrils are formed from the elaborations of the actin cytoskeleton and aggregations of $\alpha$-actinin (Myhre and Pilgrim, 2012). They then attach to the cell surface and are centered on extracellular complexes, termed costameres, constructed from premyofibril templates, which are established in the prefusion myoblasts and are composed of cortical actin and nonmuscle myosin (Pardo et al., 1983; Sparrow and Schock, 2009). As myotube maturation proceeds, additional myofibrils will fill the interior of the cell, anchored to each other as well as intracellular organelles via intermediate filament myofibrils, and eventually undergo reorganization to highly structured bundles spanning the length of the cell (Grosberg et al., 2011; Myhre and Pilgrim, 2012).

\subsubsection{Types of muscle fibers arising during embryonic myogenesis}

Embryonic and fetal myoblasts represent intrinsically disparate myogenic lineages that separately give rise to myotubes in a temporally regulated manner (Biressi et al., 2007). Consistent with this, the two types of muscle fibers that arise through fusion of myoblasts during embryonic myogenesis, the primary (embryonic) and secondary (fetal) myofibers also differ in many features. These include the expression of genes involved in skeletal muscle development and physiology, as well as the differential expression of muscle creatine kinase $(\mathrm{Ckm}), \beta$-enolase (Eno3), protein kinase theta $(\mathrm{Pkc} \theta)$ and myosin heavy chain (MyHC) isoforms (Bonner and Hauschka, 1974; Barbieri et al., 1990; Ferrari et al., 1997). This difference between embryonic and fetal myotubes is in part attributed to the activities of transcription factor Nfix, whose expression is activated by Pax7 in fetal muscles and is thought to be responsible for controlling the switch from embryonic to fetal myogenesis (Messina et al., 2010). Although myosin expression is first detected at E9 in the mouse embryo (Furst et al., 
1989), it is during secondary myogenesis that myofibers begin to differentially express distinct myosin isoforms that will discriminate the future fast- and slow-twitch muscle fibers in the adult (Lyons et al., 1990). Primary myotubes in rodents express both embryonic myosin heavy chain (MyHC-emb) and adult slow myosin heavy chain (MyHC-slow), whereas secondary myotubes express MyHC-emb and neonatal myosin heavy chain (MyHC-neo). Primary myotubes are the precursors of adult slow muscle fibers, with only a minority of them suppressing MyHC-slow and expressing adult fast myosin heavy chain (MyHC-fast) (Jansen and Fladby, 1990). Most secondary myotubes, on the other hand, develop into fast fibers.

The initial specialization into different fiber types is attributed to the innate properties of distinct myoblasts lineages, and is thought to occur largely independent from innervation, although the precise influence (or absence thereof) of innervation for embryonic muscle fiber type specification remains contested (Schiaffino et al., 2007; Rana et al., 2009). It remains unclear whether developing fibers expressing MyHCemb and/or MyHC-neo represent particular functional contractile structures or if they are just provisional transitional structures that appear before the emergence of definitive fiber types after birth (Schiaffino and Reggiani, 2011).

\subsection{Nerve-muscle interaction}

The neuromuscular system is virtually the only neural system in which the precise target and functional output of the innervating neurons, the motor neurons, are known. Given the tight relationship of motor neurons and the muscle fibers that they innervate, it is not surprising that the development and maintenance of both components require extensive forward- and reverse-crosstalk. For instance, about 60$67 \%$ of motor neurons initially generated are eliminated via programmed cell death (PCD) starting from E13 in the thoracic and lumbar spinal cord (Lance-Jones, 1982; Oppenheim, 1986; Oppenheim, 1991). The functional significance of this 
developmental elimination process remains speculative. Blocking AChRs with nicotinic blocking agents was observed to block the intrinsic apoptotic program and preserve the initial motor neuron number (Oppenheim, 1991; Hory-Lee and Frank, 1995). At the same time, nicotinic blocking agents failed to block motor neuronal cell death after removal of the limb buds, suggesting that muscle-derived factors or retrograde signals regulate motor neuron elimination during development (Pittman and Oppenheim, 1979; Caldero et al., 1998). Furthermore, experiments have shown that removal of target muscles causes motor neuron degeneration, while increasing the target size augments motor neuron survival (Hollyday and Hamburger, 1976; Oppenheim, 1991). Conversely, there is evidence to support the presence of anterograde signals provided by the motor neurons to the muscles (Altar and DiStefano, 1998).

During the development of the neuromuscular junction, AChR clusters are small and dispersed at the endplate of the muscles (median positioned). Nerve-derived signals such as agrin are known to induce maturation and maintenance of the postsynaptic AChR clusters, thus resulting in denser, larger clusters and their alignment with presynaptic motor axon terminals (Lin et al., 2001). Cross-innervation studies done by Buller et al. (1960) also demonstrated that certain properties (e.g. contractile properties) of muscles partially depended on motor innervation. While there is a broad consensus regarding the interdependence of motor neurons and muscles, the extent of this interdependence remains contested, however.

\subsubsection{Dependence of muscles on innervation}

During myogenesis, motor nerves branch extensively within muscles after the cleavage of pre-muscle mass and after most or all the primary myotubes have formed (Tosney and Landmesser, 1985b; Dahm and Landmesser, 1988; Ontell et al., 1988). The end of primary myogenesis marks innervation of primary myotubes by motor neurons 
(McLennan, 1983a; Ross et al., 1987). The nerve was initially thought to be important for the continued differentiation and survival of muscle fibers, while the initial steps of myogenesis would occur self-autonomously (Hamburger, 1939; Hamburger and Waugh, 1940; Bradley, 1970). These conclusions were based on early pioneering work by Victor Hamburger and others who performed surgical manipulations in chick embryos, including neural tube or limb extirpation and grafting experiments (Hamburger, 1939; Hamburger and Waugh, 1940; Bradley, 1970), although these experiments did not include sham-operated controls to account for potential effects of the surgery proper (Hunt, 1932; Hamburger, 1939). Nevertheless, these experiments have undeniably paved the way for much of the work that followed involving neural influence on muscle development.

\subsubsection{Primary myotubes and denervation}

It is established that the early cleavage of pre-muscle mass occurs independently of innervation (Hunt, 1932; Hamburger, 1939; Shellswell, 1977; Butler et al., 1982). However, the role of innervation in the formation of primary and secondary myofibers and the extent of interdependence between the nerve and developing muscles remain controversial. In the 1980s, it was generally accepted that primary myotubes form independently of the nervous system (McLennan, 1994). This was based on many lines of evidence that observed that primary myotubes form prior to the muscle being extensively innervated, and that a normal number of myotubes were formed in denervated muscles (Harris, 1981; Butler et al., 1982; Phillips and Bennett, 1984; Ross et al., 1987). Crow and Stockdale (1986) as well as other investigators have also shown that in the developing chick, the fiber specialization of early myotubes (primary myotubes, based on MyHC-slow expression) appears to be unaffected after dtubocurarine (d-tbc)-induced neuromuscular contact disruption or brachial spinal cord deletion (Butler et al., 1982; Phillips and Bennett, 1984; Crow and Stockdale, 
1986). However, this conclusion was eventually challenged by Fredette and Landmesser (1991), who proposed a crucial role for innervation on primary myotube formation and maintenance. This was based on using d-tbc to induce paralysis or denervation (lumbosacral spinal cord removal) in the chick embryos, upon which it was observed that the formation of primary myotubes of the iliofibularis muscle was affected in the late stage of primary myogenesis, along with degeneration of earlier formed myotubes. Additionally, this was supported by evidence from the mouse that formation of primary myotubes in the soleus muscle was prevented almost entirely after laser ablation of the spinal cord during embryonic development at E14.5 (Hughes and Ontell, 1992).

\subsubsection{Influence of innervation on secondary myotubes}

Secondary myogenesis has generally been thought to be greatly dependent on the nerve (Ross et al., 1987; Harris, 1981; McLennan, 1983; Phillips and Bennett, 1984). This conclusion was based on results obtained by using blockers ( $\beta$-bungarotoxin, tetrodotoxin and $\mathrm{d}-\mathrm{tbc}$ ) to induce muscle denervation or paralysis in avian, duck and rat embryos which revealed that secondary myotube formation was either severely hampered or completely abolished in these conditions (Harris, 1981; McLennan, 1983b; Phillips and Bennett, 1984; Sohal and Sickles, 1986; Ross et al., 1987; Wilson and Harris, 1993). These observations were in conflict with results obtained by several other studies, which concluded that a large number of secondary myotubes could be observed even after paralysis or denervation (Condon et al., 1990; Fredette and Landmesser, 1991a; Hughes and Ontell, 1992). As a result, two main but disparate hypotheses have arisen: (1) the first advocating that a subset of late-forming primary myotubes is nerve-dependent, whereas secondary myotubes form independently of innervation, but instead rely on the surviving primary myotubes to provide scaffolds for their formation (Condon et al, 1990; Fredette and Landmesser, 1991), (2) whereas 
the second proposed that primary myotube formation is a cell-autonomous event, but secondary myogenesis depends on the regulatory or permissive effect of innervation (Ross et al., 1987; Harris, 1981; Butler, 1982; Phillips and Bennett, 1984). Hence, to date, whether the presence of the motor nerve is required for the formation of primary and/or secondary myotubes remains unresolved. Furthermore, it is unclear if primary and secondary myotubes display differential responses to denervation and if they have distinct requirements from the nerve (e.g. different degree of dependence) for their formation and maintenance.

\subsubsection{Nerve aspects that could regulate muscle development}

Even though secondary myogenesis is generally accepted to be dependent on the nerve (Ross et al., 1987; Harris, 1981; McLennan, 1983; Phillips and Bennett, 1984), the functional significance of the initial interaction between the nerve and muscle with regards to myotube formation is still poorly understood. Administration of d-tbc to chick embryos before the establishment of nerve-muscle contact has been found to abolish secondary myotube formation (Sohal and Sickles, 1986; McLennan, 1983c). In contrast, administration of the same drug before neuromuscular terminal formation had no effect on secondary myogenesis (Dahm and Landmesser, 1988). Similarly, denervation in rodent muscles by $\beta$-bungarotoxin $(\beta$-BTX) after the nerve has entered the muscle results in secondary myotube formation, whereas denervation before the nerve has entered the muscle results in a profound inhibition of secondary myogenesis (Harris, 1981; Ross et al., 1987; Wilson and Harris, 1993). In brief, earlier studies have reported that the extent of secondary myogenesis inhibition appears to correlate with the time of physical and chemical denervation, such that earlier treatment had a more pronounced effect compared to later treatment on embryos (Harris, 1981; Phillips and Bennett, 1984; Ross et al., 1987; Condon et al., 1990). Thus, although nerve-muscle interaction seemed to influence myogenesis, it is less clear as to 
which specific aspect of the nerve (i.e. nerve contact, nerve activity, or nerve-derived neurotrophic factors) would predominantly be required for secondary myotube formation.

\subsection{Spontaneous motility and nerve activity}

A common feature during nervous system development is the occurrence of rhythmic spontaneous episodes of activity which is thought to be highly relevant for neuronal circuit formation and fine-tuning, especially in circuits involved in motor pattern generation (Hanson and Landmesser, 2003). Spontaneous motility in vertebrates is generated approx. 1 day after the innervation of muscles by spontaneously active spinal motor neurons at the appropriate developmental stage (Moody and Bosma, 2005). In the mouse, spontaneous activity in the developing motor neurons was predominantly recorded in lumbar motor nerves in E11.4-14.5 spinal cord (Hanson and Landmesser, 2003). By E12.5, spontaneous motility, which is propagated in a rostral-to-caudal fashion, could be detected as small random movements in the mouse (i.e. $70 \%$ of the movements is initiated near the head) before proceeding to a highly patterned motility with alternative flexor and extensor motor output as well as activity that alternates between the two sides of the body as development progresses (Moody and Bosma, 2005). Further, E15 is thought to be the stage which corresponds to a transitional period in the maturation of rhythmic patterns during which two disparate rhythms are expressed, as development of a more mature phenotype (characterized by irregular short-duration events) that likely reflects the emergence of evoked nerve activity in the embryo (Yvert et al., 2004).

The relative conservation of the initiation of spontaneous activity between the chick and mouse suggests that spontaneous activity is important to development in general (Moody and Bosma, 2005). Because spontaneous activity persists throughout the period of innervation with evolving patterns of activity, muscle and neuronal 
differentiation are likely to be influenced by changes in these pattern of activity as development progresses (Moody and Bosma, 2005). In brief, spontaneous motor neuron activity is known to be crucial for many aspects of development, such as the pruning of supernumerary inputs to the NMJ, matching axons with target motor units, and most importantly, facilitating the increase of intracellular $\mathrm{Ca}^{2+}$ in myoblasts in the absence of action potentials, thereby triggering their fusion (Moody and Bosma, 2005). Mice deficient for $\gamma$-subunit of AChR failed to develop spontaneous muscle action potentials (triggered by miniature end plate potentials), exhibited lower density of cross-striation in muscle fibers and died within two days after birth, suggesting that spontaneous activity may play additional roles in myogenesis (Takahashi et al., 2002). Unfortunately, the extent to which spontaneous activity might promote muscle formation and/or maintenance during embryogenesis and the critical stage for this requirement remains elusive.

\subsubsection{Evoked and spontaneous vesicular release}

Neurons communicate primarily by releasing neurotransmitters stored in small organelles of similar size and shape, known as synaptic vesicles (Jahn and Sudhof, 1994). Two types of synaptic vesicular release can be observed at the neuromuscular synapse. One is the less well-understood action potential-independent spontaneous transmitter release, first discovered by Bernard Katz and colleagues (Kavalali et al., 2011). This typically occurs with a low probability and involves fusion of a single synaptic vesicle (Fatt and Katz, 1952; Katz and Miledi, 1964). On the other hand, evoked (action potential-dependent) transmitter release is elicited by a $\mathrm{Ca}^{2+}$ influx (Deitcher et al., 1998). Under physiological conditions, the unitary synaptic transmission events (in muscles, end plate potential- EPP) that occur from the fusion of individual synaptic vesicles are to a large extent similar to one another in terms of their kinetics and the population of target receptors that they activate, regardless of 
whether vesicles were exocytosed spontaneously or induced by action potentials (Kavalali et al., 2011). Traditionally, these two types of vesicular release were thought to have similar exocytotic apparatus, differing only in their rate-limiting response to different $\mathrm{Ca}^{2+}$ concentrations. However, recent studies on neuronal SNAREs (soluble $\mathrm{N}$-ethylmaleimide-sensitive factor attachment protein receptors) consisting of SNAP25, synaptobrevin (also known as VAMP2) and syntaxin1 that make up the core multiprotein complex (Jahn and Scheller, 2006; Sudhof and Rothman, 2009) revealed differences underlying the two types of vesicular release and suggested that they utilize distinct molecular interactions of the same fusion machinery (Kavalali et al., 2011). Synaptobrevin, the protein involved in vesicle docking and/or fusion and a substrate for tetanus toxin (TeTx), was interestingly found to abolish $\mathrm{Ca}^{2+}$-dependent evoked release completely, whereas the rate of spontaneous release of a single vesicle persists albeit being reduced (Deitcher et al., 1998; Schoch et al., 2001).

\subsubsection{Acetylcholine (ACh)}

Increase in intracellular $\mathrm{Ca}^{2+}$ induced by nerve activity can trigger the secretion of developmentally active molecules, including neurotransmitters, that could play roles during different developmental processes (Moody and Bosma, 2005). ACh is the main neurotransmitter in the vertebrate motor system and it underlies both spontaneous and evoked synaptic activity at all vertebrate NMJs (Misgeld et al., 2002). As such, neurotransmission is the only established role for ACh in the nervous system, although it has long been hypothesized to be involved as a regulator in myogenesis based on the effect on muscles after neurotransmission blockade (or in vitro data) (Drachman and Coulombre, 1962; Drachman, 1964; Giacobini et al., 1973; Hall, 1975; Entwistle et al., 1988; Krause et al., 1995). From these studies, neuromuscular transmission and resulting muscle activity were thought to constitute trophic influences necessary for the prevention of denervation changes in the muscles. 
However, later studies suggest that not all effects of the nerve on muscles might be mediated by ACh; paralysis of the rat IV lumbrical muscle by TTX (Ross et al., 1987), duck superior oblique muscle by $\alpha$-BTX (Sohal and Holt, 1980) and chick iliofibularis muscle by d-tbc (McLennan, 1983b) was found to result in lesser degeneration and a prolonged survival of primary myotubes when compared to denervation of these muscles by $\beta$-BTX or spinal cord segment removal. Based on this observation, synaptic ACh was suggested to upkeep primary myotubes not by acting as a sole factor, but instead by acting in combination with other factors (McLennan, 1994). This idea was well supported by the finding that constant electrical stimulation of aneural chick PLD (posterior latissimus dorsi) muscle delays their degeneration, but fails to reinstate muscles to their normal size (Bloom et al., 1985). Knocking out the Chat gene, which encodes for the choline acetyltransferase enzyme used in the biosynthesis of ACh, revealed hyperinnervation of muscles and synaptic maturation defects (Misgeld et al., 2002). More importantly, diaphragm muscles were considerably thinner with fewer muscle fibers in the Chat mutants, suggesting that ACh-mediated neurotransmission might be a crucial myogenic requirement for myotube formation and/or survival (Misgeld et al., 2002).

\subsection{Adult denervation pathway}

Fiber type diversification in the embryos is thought to occur independently of neural influence, as shown by experiments using $\beta$-BTX to destroy motor neurons in rat fetuses prior to muscle innervation (Condon et al., 1990). Although muscle growth was drastically reduced by the loss of motor neurons, muscles of fetuses still contained fibers expressing either MyHC-neo or MyHC-slow with differential distribution. However, in postnatal animals, neuronal activity is one of the most important aspects in maintaining fiber type profile of skeletal muscles (Schiaffino and Reggiani, 2011). The role of innervation in muscle fiber type differentiation is evident in the postnatal 
development of slow muscle fibers: for example, postnatal denervation of the rat soleus resulted in the progressive decline and eventual disappearance of slow myosin 4 weeks after denervation (Gambke et al., 1983).

To maintain homeostasis, a balance between anabolic (synthetic) and catabolic (degenerative) processes is necessary. During embryonic myogenesis, myotubes grow in length, resulting from the end-to-end fusion of myoblasts to form multinucleated myotubes (Zhang and McLennan, 1995). More importantly, muscle growth during embryonic development is characterized by an increase in fiber numbers, generated from the two waves of myogenesis (primary and secondary). Therefore, embryonic muscle growth is principally dependent on the precise limitation of differentiation, which is executed by proliferative and Pax3-promoting signals such as BMP4 (Amthor et al., 1999). Conversely, subsequent growth in postnatal muscle fibers is due to hypertrophy (an increase in muscle fiber size), with no net formation of new muscle fibers. An exception to this is during muscle regeneration, when satellite cells are activated to replace injured muscle fibers (Lepper et al., 2011). Muscle growth in postnatal muscle is achieved either by increased protein content, decreased protein breakdown, or both (Boonyarom and Inui, 2006), and is regulated primarily by components in the highly conserved signaling pathway, the insulin-like growth factor 1-Akt/protein kinase B (IGF1-Akt/PKB) pathway (Schiaffino and Mammucari, 2011).

Contrastingly, aging, starvation, pathologic states, inactivity or denervation can lead to muscle atrophy (decrease in total muscle mass). In the adult, denervation-induced atrophy occurs primarily through ubiquitin-mediated proteolysis via the proteasome (Beehler et al., 2006), although muscle atrophy in general can be governed by at least five proteolytic pathways, including the lysosomal, $\mathrm{Ca}^{2+}$-dependent, caspase systems, matrix metalloproteinases and the ubiquitin proteasome pathway (Ventadour and Attaix, 2006). During atrophy, the muscle cell undergoes degeneration with structural changes including sarcomere dissolution, endothelial degradation (Oki et al., 1995) and cellular 
changes, such as reduction of mitochondria numbers (Rifenberick et al., 1973), accumulation of connective tissue and the loss of myonuclei by apoptosis (Oki et al., 1995).

Through the years many factors have been associated with adult denervation, most importantly including the E3 ubiquitin ligases, Muscle RING Finger 1 (MuRF1) and Atrogin-1/Muscle Atrophy F-box (MAFbx), which are effectors known to mediate atrophy-related degradation triggered by denervation and are established as universal markers of atrophy (Bodine et al., 2001; Ventadour and Attaix, 2006). In five models of atrophy namely: denervation, immobilization, unweighting, treatment with cachectic cytokine interleukin-1 and treatment with glucocorticoid dexamethasone (Goldberg, 1969; Bodine et al., 2001; Moresi et al., 2010; Macpherson et al., 2011), the two genes encoding E3 ubiquitin ligases were found to be upregulated and overexpression of Atrogin-1 in differentiated C2C12 skeletal myotubes produced atrophy (Bodine et al., 2001). Conversely, knocking out either MuRF1 or Atrogin-1 conferred a resistance to neurogenic atrophy (atrophy resulting from denervation) (Bodine et al., 2001). Until recently, it was unclear whether muscle atrophy induced by different triggers is governed by a common signaling pathway or whether several pathways exist that can result in muscle atrophy (Bodine et al., 2001; Moresi et al., 2010). In 2010, work done by two independent groups has shed light on part of the signaling ensemble underlying adult denervation and found that myogenin, a member of the MRF family, induces neurogenic atrophy (Moresi et al., 2010; Macpherson et al., 2011). During denervation in the adult, muscle fibers appear to regress to an immature state by re-expressing some of MRFs, which are normally downregulated either during or after embryonic myogenesis (Merlie et al., 1994; Williams et al., 2009). Myogenin was found to be a key regulator of MuRF1 and Atrogin-1 expression, two effectors responsible for neurogenic atrophy of muscle fibers. Denervation-induced myogenin upregulation appears to be in turn controlled by class IIa histone deacetylases (Hdacs) 4 and 5. Consistent with this, mice lacking Hdac 4 and 5 in skeletal muscle failed to upregulate myogenin and appear to be 
resistant to neurogenic atrophy (Moresi et al., 2010). It has been shown that Hdac 4 and Hdac 5, which are able to act as transcriptional repressors (Haberland et al., 2009), are upregulated in response to denervation and negatively regulate expression of Dach2 (Cohen et al., 2007), a known transcriptional repressor of myogenin. In contrast, knowledge about signaling pathways involved during embryonic denervation is far less well characterized, in part due to the technical difficulties in manipulating embryos to create a nerve-free myogenic environment.

\subsection{Aim of the project}

Despite a plethora of classical embryological and physiological studies that have investigated the interaction between motor neurons and muscle fibers during development, the extent to which innervation contributes to embryonic myogenesis have long been controversial, while the underlying mechanisms remain elusive (Butler et al., 1982; McLennan, 1983b; Ross et al., 1987; Condon et al., 1990; Fredette and Landmesser, 1991a; Hughes et al., 1992; Wilson and Harris, 1993). Because of technical difficulties and/or variations in creating aneural and paralyzed muscles to study the role innervation plays during embryonic myogenesis, data obtained from these studies have led to two main yet divergent conclusions on the myogenic requirements from the nerve as well as the myogenic response to denervation.

Hence, the initial aim of this project was to revisit the interdependence between muscles and motor neurons during development through selective gene manipulation in the mouse. To achieve this, a combination of transgenic mouse lines was employed to either create aneural embryonic muscles by wholesale removal of motor neurons from the onset of their formation or to produce paralyzed embryonic muscles by overexpressing TeNT specifically in the motor neurons or inactivating Chat to block motor neurotransmission. In using and carefully controlling this genetic approach, pleiotropic effects that arise from manual surgery or whole-embryo application of 
drugs can be circumvented, thus facilitating the selective in-depth analysis of the role motor innervation has during embryonic myogenesis. Ultimately, the aim of this work was to identify molecular pathways regulated by innervation to drive embryonic myogenesis and/or maturation and maintenance of muscle fibers. The discovery of genes involved in embryonic innervation-dependent myogenic programs may ultimately provide insights into degenerative neuromuscular diseases with a prenatal onset. 


\section{Materials and methods}

\subsection{Consumables and plastic ware}

Lab consumables were from Starlab GmbH (Hamburg), Eppendorf AG (Hamburg) and Sarstedt AG (Nürnstedt). Dissection instruments were purchased from Fine Science Tools GmbH.

\subsubsection{Antibodies}

\begin{tabular}{|c|c|c|c|c|}
\hline \multicolumn{5}{|l|}{ Primary antibodies } \\
\hline Antigen & Host & Dilution & Code & Supplier \\
\hline $\begin{array}{l}\text { Cleaved } \\
\text { caspase-3 }\end{array}$ & Rabbit & 1:1000 & $5 \mathrm{~A} 1 \mathrm{E}$ & Cell signaling $\mathrm{GmbH}$ \\
\hline Desmin & Mouse & $1: 1000$ & D-33 & DAKO GmbH \\
\hline Fast myosin & Mouse & $1: 200$ & F59 & Developmental Studies \\
\hline & & & & Hybridoma Bank \\
\hline General myosin & Mouse & $1: 200$ & MF20 & Developmental Studies \\
\hline & & & & Hybridoma Bank \\
\hline GFP & Rabbit & $1: 2000$ & A-11122 & Life Technologies $\mathrm{GmbH}$ \\
\hline Islet $1 / 2$ & Rabbit & $1: 2500$ & Isl $1 / 2$ & Gift from S.L. Pfaff \\
\hline Islet $1 / 2$ & Mouse & $1: 200$ & 39.4D5 & Developmental Studies \\
\hline & & & & Hybridoma Bank \\
\hline Laminin & Rabbit & $1: 500$ & L9393 & Sigma Aldrich AG GmbH \\
\hline Myf5 & Rabbit & $1: 200$ & C-20 & Santa Cruz Biotech $\mathrm{GmbH}$ \\
\hline Neonatal myosin & Mouse & $1: 4000$ & My-32 & Sigma Aldrich AG GmbH \\
\hline NeuN & Mouse & $1: 1000$ & MAB377 & Merck Millipore $\mathrm{GmbH}$ \\
\hline Neuronal nuclei & Mouse & $1: 1000$ & NeuN & Merck Millipore GmbH \\
\hline NF (160 kDa) & Mouse & $1: 200$ & $4 \mathrm{H} 6$ & Developmental Studies \\
\hline & & & & Hybridoma Bank \\
\hline Parvalbumin & Rabbit & $1: 2000$ & PV & Covance $\mathrm{GmbH}$ \\
\hline
\end{tabular}




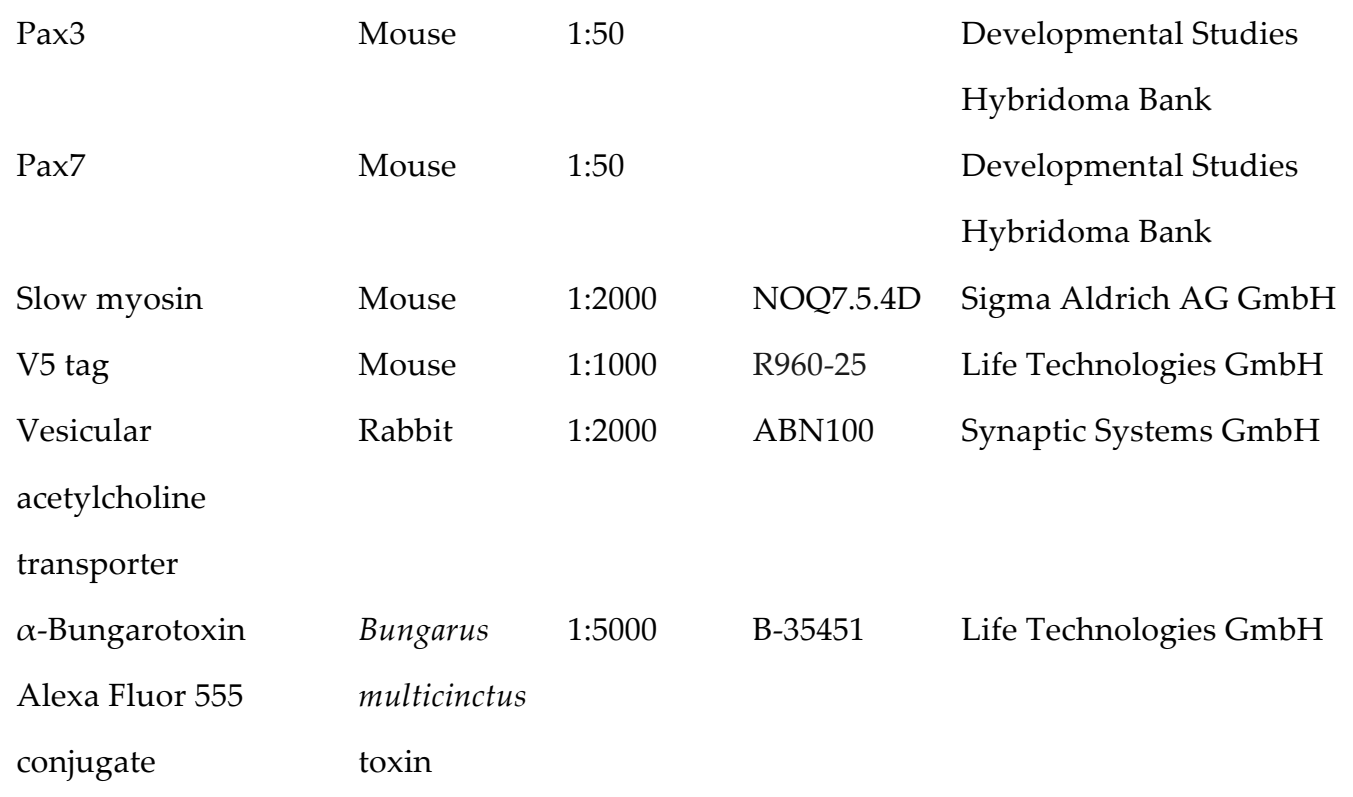

\begin{tabular}{|c|c|c|c|c|}
\hline \multicolumn{5}{|c|}{ Secondary antibodies and nuclei dye } \\
\hline Target & Conjugate & Host & Dilution & Supplier \\
\hline Mouse & Alexa fluorescent dye & Donkey & 1:2000 & Life Technologies GmbH \\
\hline Rabbit & Alexa fluorescent dye & Donkey & 1:2000 & Life Technologies GmbH \\
\hline Sheep & Alexa fluorescent dye & Donkey & $1: 2000$ & Life Technologies GmbH \\
\hline Nuclei dye & DAPI & & 1:1000 & Sigma Aldrich AG GmbH \\
\hline
\end{tabular}

\subsubsection{Enzymes}

\begin{tabular}{ll}
\hline Enzyme & Supplier \\
\hline DNA Ligation Kit & Takara Bio Inc \\
Dream Taq DNA Polymerase & Thermo Fisher Scientific GmbH \\
Phusion High Fidelity DNA Polymerase & New England Biolabs GmbH \\
Platinum Taq DNA Polymerase High Fidelity & Life Technologies GmbH \\
Restriction enzymes & Thermo Fisher Scientific GmbH
\end{tabular}


Shrimp Alkaline Phosphatase

SP6 RNA Polymerase

T4 DNA Ligase

T4 Polynucleotide Kinase

T7 RNA Polymerase
Thermo Fisher Scientific GmbH

Roche Diagnostics GmbH

Thermo Fisher Scientific GmbH

Thermo Fisher Scientific GmbH

Roche Diagnostics GmbH

\subsubsection{Kits}

\begin{tabular}{ll}
\hline Kit & Supplier \\
\hline Antigen Unmasking Solution Kit & Vector Laboratories Inc \\
cDNA synthesis Kit & Roche Diagnostics GmbH \\
Click-iT EdU Alexa Fluor Imaging Kit & Life Technologies GmbH \\
Illumina Total Prep RNA Amplification Kit & Life Technologies GmbH \\
PrimeScript First Strand cDNA Synthesis Kit & Takara Bio Inc \\
QIAGEN Plasmid Maxi Kit & Qiagen GmbH \\
QIAprep Spin Miniprep Kit & Qiagen GmbH \\
QIAquick Gel Extraction Kit & Qiagen GmbH \\
Taqman Universal Probe Library (mouse) & Roche Diagnostics GmbH \\
TOPO TA Cloning Kit & Life Technologies GmbH
\end{tabular}

\subsubsection{Chemicals and reagents}

\begin{tabular}{ll}
\hline Chemical & Supplier \\
\hline Agar-Agar & Carl Roth GmbH \\
Agarose NEEO & Carl Roth GmbH \\
Ammonium acetate & Carl Roth GmbH \\
Ampicillin & Carl Roth GmbH \\
Benzyl alcohol (BA) & Carl Roth GmbH \\
Benzyl benzoate (BB) & Sigma-Aldrich AG GmbH \\
Blocking reagent & Roche Diagnostics $\mathrm{GmbH}$
\end{tabular}


Bovine serum albumin fraction V (BSA)

Calcium chloride

Chloroform

Cupric sulfate

D-PBS pH 7.2

Diethylpyrocarbonate (DEPC)

Dimethyl sulfoxide (DMSO)

DNA Ladder GeneRuler 1kb

DNA loading dye 6x

dNTP Mix

Ethanol $99.9 \%$

Ethidium bromide solution $1 \%$

Ethylenediaminetetraacetatic acid (EDTA)

Fast green

Glacial acetic acid

Glucose

Glutaraldehyde

Glycerol

Hydrochloric acid

Isopropanol

L-15 medium Leibowitz

Magnesium chloride

Methanol

OCT embedding medium

Paraffin pellets

Paraformaldehyde (PFA)

PBS pH 7.2

Phenol-Chloroform

Polyacrylamide

Potassium chloride

Potassium hydroxide
Carl Roth $\mathrm{GmbH}$

Carl Roth $\mathrm{GmbH}$

Carl Roth $\mathrm{GmbH}$

Carl Roth $\mathrm{GmbH}$

Life Technologies $\mathrm{GmbH}$

Carl Roth $\mathrm{GmbH}$

Sigma-Aldrich AG GmbH

Thermo Fisher Scientific GmbH

Thermo Fisher Scientific GmbH

Thermo Fisher Scientific GmbH

Carl Roth $\mathrm{GmbH}$

Carl Roth $\mathrm{GmbH}$

Carl Roth $\mathrm{GmbH}$

Sigma-Aldrich AG GmbH

Carl Roth $\mathrm{GmbH}$

Sigma-Aldrich AG GmbH

Sigma-Aldrich AG GmbH

Carl Roth $\mathrm{GmbH}$

Carl Roth $\mathrm{GmbH}$

Carl Roth $\mathrm{GmbH}$

Sigma-Aldrich AG GmbH

Carl Roth $\mathrm{GmbH}$

Carl Roth $\mathrm{GmbH}$

Sakura Finetek GmbH

Carol Roth GmbH

Carl Roth $\mathrm{GmbH}$

Life Technologies $\mathrm{GmbH}$

Carl Roth $\mathrm{GmbH}$

Carl Roth $\mathrm{GmbH}$

Carl Roth $\mathrm{GmbH}$

Carl Roth $\mathrm{GmbH}$ 
Proteinase K

Sodium acetate

Sodium chloride

Sodium citrate

Sodium dodecyl sulfate (SDS)

Sodium hydroxide

Sucrose

Tris- $\mathrm{HCl}$

Triton X-100

TRIzol reagent

Trypsin

Tryptone

Tween-20

Xylene

Yeast extract

1-hexadecane

$\beta$-Mercaptoethanol
AppliChem GmbH

Carl Roth $\mathrm{GmbH}$

Carl Roth $\mathrm{GmbH}$

Carl Roth $\mathrm{GmbH}$

Carl Roth $\mathrm{GmbH}$

Carl Roth $\mathrm{GmbH}$

Carl Roth $\mathrm{GmbH}$

Carl Roth $\mathrm{GmbH}$

Carl Roth $\mathrm{GmbH}$

Life Technologies $\mathrm{GmbH}$

Life Technologies $\mathrm{GmbH}$

Carl Roth $\mathrm{GmbH}$

Carl Roth $\mathrm{GmbH}$

Carl Roth $\mathrm{GmbH}$

AppliChem GmbH

Sigma-Aldrich AG GmbH

Carl Roth $\mathrm{GmbH}$

\subsubsection{Solutions}

\begin{tabular}{ll}
\hline Solution & Reagents \\
\hline Antibody staining solution & PBS pH 7.2; 1\% BSA; 1\% Triton X-100 \\
BABB clearing solution & $66 \%$ Benzyl benzoate; 34\% Benzyl alcohol \\
Cupric sulfate solution & pH 5.0; 10 mM Cupric sulfate; 50 mM Ammonium acetate \\
Dent's solution & $80 \%$ Methanol; 20\% DMSO \\
LB agar & LB medium; $\%$ [w/v] Agar-Agar \\
LB medium & 5 g/l Sodium chloride; 5 g/l Yeast extract; 10 g/l Tryptone \\
Mounting solution & PBS pH 7.2; 50\% Glycerol \\
PBST & PBS pH 7.2; 0.1\% Tween-20 \\
PFA solution & PBS pH 7.2; $4 \%$ [w/v] Paraformaldehyde
\end{tabular}


Sucrose solution

TAE buffer

Tail biopsy lysis buffer
PBS pH 7.2; 30\% [w/v] Sucrose

$40 \mathrm{mM}$ Tris; $1 \mathrm{mM}$ EDTA adjust $\mathrm{pH} 7.5$ with glacial acetic acid

$0.1 \mathrm{M}$ Tris $\mathrm{pH}$ 8.0; $30 \mathrm{mM} \mathrm{NaCl} ; 0.2 \mathrm{mM}$ EDTA; 0.05\% SDS;

$0.5 \mathrm{mg} / \mathrm{ml}$ Proteinase K

\subsubsection{Vectors}

\begin{tabular}{ll}
\hline Plasmid & Supplier \\
\hline$p 2 T K-C M V-2 A-e G F P$ & Workgroup Marquardt \\
$p 2 T K-M L C-E G F P-F$ & Gift from D. Duprez (Wang et al., 2011) \\
$p C A G G S-T 2 T P$ & Gift from K. Kawakami (Sato et al., 2007) \\
$p C R I I-T O P O$ & Life Technologies GmbH \\
$P G K d t a b p A$ & Addgene Inc
\end{tabular}

\subsubsection{Software}

\begin{tabular}{lll}
\hline Software & Application & Supplier \\
\hline Adobe Creative Suite CS5 & Image processing & Adobe Systems Inc \\
Bead Studio & Microarray data analysis & Illumina Inc \\
DAVID & Microarray data analysis & http://david.abcc.ncifcrf.gov \\
ImageJ & Image quantification & http://rsbweb.nih.gov/ij/ \\
Microsoft Office 2007 & Data input & Microsoft Corp \\
Primer3 & Primer design & http://primer3.sourceforge.net \\
Prism 5.0 & Statistical analysis & GraphPad Software Inc \\
Vector NTI & DNA sequence analysis & Life Technologies GmbH
\end{tabular}




\subsection{Chick embryos}

Lohmann LSL strain fertilized eggs (Horstmann Geflügelzucht $\mathrm{GmbH}$ ) were used. Before electroporation, they were incubated (Hemel-Brutgeräte) for $65 \mathrm{~h}$ at $37.8^{\circ} \mathrm{C}$ and $80 \%$ humidity.

\subsection{Mouse lines}

All animal experiments have been carried out in accordance with the animal protection law of the Federal Republic of Germany and were approved by the district government. Animals were housed under standard conditions in the institute's animal facility with access to food and water ad libitum. When a vagina plug was confirmed on the morning after mating on the previous evening, the period of pregnancy was designated at 0.5 post-conception $(\mathrm{dpc})$. All stages of embryos in this study were obtained by cervical dislocation, and analyzed by routine histological and molecular biological procedures.

\subsubsection{R26 $6^{f D T A} x O \operatorname{Oig} 2^{\text {Cre }}$ mice}

Olig2 is a bHLH transcription factor essential for driving the differentiation of motor neurons and at later stages, oligodendrocytes (Takebayashi et al., 2002). Olig2 ${ }^{\mathrm{Cre}}$ mice which carry an insertion of Cre expressed under control of Olig2 promoter were bred to the Rosa2 $6^{f x D T A}\left(R 26^{f x D T A}\right)$ strain to conditionally ablate premitotic motor neurons. $R 26^{f x D T A}$ carries an insertion of the Diptheria toxin subunit $A$ (DTA) gene in the ubiquitously expressed Rosa26 locus (Ivanova et al., 2005). Transcription of the R26fxDTA allele ceases prematurely in $R 26^{f x D T A} x$ Olig2 $2^{\text {Cre }}$ mice because of a loxP flanked (floxed) transcriptional termination sequence before the DTA sequence. Cre-mediated recombination cleaves the floxed termination sequence and drives DTA expression, thereby resulting in conditional cell death of Cre-expressing premitotic 
motor neuron progenitors. Cell specific ablation is brought about by catalyzation of ADP ribosylation of eukaryotic elongation factor 2 by DTA.

\subsubsection{Isl2 ${ }^{\text {fxDTA }} x \mathrm{Olig} 2^{\mathrm{Cre}}$ mice}

Olig2 ${ }^{C r e}$ mice were bred to Islet2 $2^{f x D T A}\left(I s l 2^{f x D T A}\right)$ strain which carries a targeted insertion of the Diptheria toxin subunit A (DTA) cassette into the sequences encoding the 3' UTR of the Insulin related protein 2 (Isl2) gene. Isl2 is one of the first homeodomain proteins to be expressed in the postmitotic motor neurons. Cre-mediated recombination drives DTA expression specifically in the postmitotic motor neurons, resulting in their cell death.

\subsection{3. $\quad \mathrm{R}^{2} 6^{\mathrm{fTeNT}} x \mathrm{Olig} 2^{\mathrm{Cre}}$ mice}

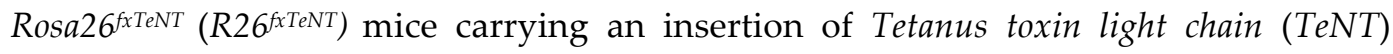
fragment expressed under control of the ubiquitous promoter Rosa26 (Zhang et al., 2008), a kind gift from Prof. Martyn Goulding (The Salk Institute), were bred to the Olig2 ${ }^{\mathrm{Cre}}$ strain to conditionally block neurotransmission in motor neurons. To generate R26 ${ }^{f x T N T} x$ Olig2 ${ }^{\text {Cre }}$ mice, the lacZ sequences downstream of PGKneo- $t P A$ cassette in the Rosa26 locus (Soriano, 1999) were replaced with a sequence encoding a GFP-TeNT fusion protein (Yamamoto et al., 2003). The transcription of the R26fxTeNT allele ceases prematurely in $R 26^{f x T e N T}$ mice because of a loxP flanked (floxed) termination sequence upstream of the TeNT sequence. Cre-mediated recombination cleaves the floxed termination sequence and drives TeNT expression, thereby resulting in conditional neurotransmission blockade of Cre-expressing motor neurons. 


\subsubsection{Chat null mice}

Choline acetyltransferase (Chat) null mice were obtained from Prof. Samuel Pfaff (The Salk Institute) as part of a collaboration. Chat is a critical enzyme responsible for the biosynthesis of acetylcholine ( $\mathrm{ACh}$ ), the main neurotransmitter in the neuromuscular system. Chat is encoded by a large gene that includes 14 coding exons in the 3' region which are used in all transcript variants (Brandon et al., 2003). The catalytic portion of the protein that affects the efficiency of ACh synthesis was targeted for deletion. A targeting vector containing a neomycin resistance cassette expressed in the opposite orientation from Chat was used to replace exons 11, 12, and 13 (Ohno et al., 2001).

\subsection{5. $\quad R 26^{f x D T A} x M y o g^{C r e}$ mice}

Myog ${ }^{\text {Cre }}$ mice carry an insertion of Cre recombinase expression cassette expressed under control of $1.5-\mathrm{kb}$ mouse myogenin promoter and the $1.0-\mathrm{kb}$ mouse MEF2C enhancer ( $\mathrm{Li}$ et al., 2005). Myogenin is a member of the MRFs and is expressed in differentiating myoblasts. Myog ${ }^{C r e}$ mice were bred to Rosa26 fxDTA (R26fxDTA $)$ mice to specifically ablate myogenin-expressing myoblasts. Cre-mediated recombination cleaves the floxed termination sequence and drives DTA expression specifically in myogenin-expressing myoblasts, resulting in their cell death, and thereby preventing muscle formation (muscle loss seen first at E14.5) (Gensch et al., 2008).

\subsubsection{Myf5-/-;MyoD-/-:Hb9-GFP mice}

Hb9-GFP is a transgenic mouse line expressing the enhanced green fluorescent protein (eGFP) under control of the $\mathrm{Hb9}$ promoter, enabling the genetic labeling of postmitotic motor neurons (Lee et al., 2004). This mouse line was crossed with Myf5+/-;MyoD+/mice described in (Rudnicki et al., 1993). 


\subsection{Genotyping}

Tail biopsies of mice were incubated in DNA lysis buffer at $56{ }^{\circ} \mathrm{C}$ overnight. Thereafter, the solution was centrifuged for $10 \mathrm{~min}$ at 12000 revolutions per minute (rpm), and the supernatant was collected and mixed with $500 \mu \mathrm{l}$ isopropanol. The mixture was centrifuged again for $10 \mathrm{~min}$ at $12000 \mathrm{rpm}$. Thereafter, the supernatant was discarded and the pellet obtained was washed with $70 \%$ ethanol by centrifugation for $5 \mathrm{~min}$ at $12000 \mathrm{rpm}$. The pellet was left to air-dry before dissolving in $50 \mu \mathrm{l}$ of distilled water and incubating at $37{ }^{\circ} \mathrm{C}$ for a minimum of $1 \mathrm{~h}$ before use for PCR (polymerase chain reaction).

\section{PCR reaction set up}

\begin{tabular}{ll}
\hline Component & Amount $(\boldsymbol{\mu l})$ \\
\hline DNA & 1 \\
dNTPs & 0.5 \\
Forward primer & 0.5 \\
Reverse primer & 0.5 \\
Taq polymerase & 0.25 \\
Double distilled water & 19.75
\end{tabular}

\section{Conditions for Mastercycler epGradient S (Eppendorf AG)}

\begin{tabular}{ll}
\hline Condition & Time \\
\hline Initial denaturation & $3 \mathrm{~min}$ \\
$94^{\circ} \mathrm{C}$ & \\
Extension 28 cycles & $1 \mathrm{~min}$ \\
$94{ }^{\circ} \mathrm{C}$ & $1 \mathrm{~min}$ \\
$62{ }^{\circ} \mathrm{C}$ & $30 \mathrm{sec}$ \\
$72{ }^{\circ} \mathrm{C}$ & \\
Final elongation & $5 \mathrm{~min}$
\end{tabular}


Primers used for genotyping

\begin{tabular}{|c|c|}
\hline Mouse allele & Primer \\
\hline \multirow[t]{2}{*}{ Rosa WT } & F: AAAGTCGCTCTGAGTTGTTAT \\
\hline & R: GGAGCGGGAGAAATGGATATG \\
\hline \multirow[t]{2}{*}{ Rosa Mutant } & F: AAAGTCGCTCTGAGTTGTTAT \\
\hline & R: GCGAAGAGTTTGTCCTCAACC \\
\hline \multirow[t]{2}{*}{ Isl 2 WT } & F: GCCATGAGAACGCGGTGCAGGGC \\
\hline & R: CGGAGTCTCCAGTCTCAGCGGTGC \\
\hline \multirow[t]{2}{*}{ Isl 2 Mutant } & F: ACGACGCTGCGGGATACTCT \\
\hline & R: CAACGCTAGAACTCCССТCA \\
\hline \multirow[t]{2}{*}{ TeNT WT } & F: AAAGTCGCTCTGAGTTGTTAT \\
\hline & R: GGAGCGGGAGAAATGGATATG \\
\hline \multirow[t]{2}{*}{ TeNT Mutant } & F: AAAGTCGCTCTGAGTTGTTAT \\
\hline & R: CATCAAGGAAACCCTGGACTACTG \\
\hline \multirow[t]{2}{*}{ Olig Cre } & F: TAAAGATATCTCACGTACTGACGGTG \\
\hline & R: TCTCTGACCAGAGTCATCCTTAGC \\
\hline \multirow[t]{2}{*}{ MyoG Cre } & F: TGTGCAGCAACAGCTTAGAG \\
\hline & R: AGGCTAAGTGCCTTCTCTACAG \\
\hline Myf5+/-;MyoD+/-:HB9-GFP & Phenotype obvious; genotyping not performed \\
\hline Myf5-/-; MyoD-/-:HB9-GFP & Phenotype obvious; genotyping not performed \\
\hline Chat & $\begin{array}{l}\text { A phosphoglycerate kinase-neomycin (pgk-neo)- } \\
\text { specific oligonucleotide, a Chat exon } 13 \text { oligonucleotide, } \\
\text { and a shared Chat intronic oligonucleotide was used for } \\
\text { genotyping (Brandon et al., 2003). }\end{array}$ \\
\hline
\end{tabular}




\subsection{Immunohistochemistry}

Mice were sacrificed on desired post-conception day (dpc). Embryos were freed from uterus of the mother and eviscerated in ice cold PBS. They were fixed in $4 \%$ PFA with conditions listed below. After fixation, embryos were washed with PBS overnight on a shaker. From this step, embryos were either processed for either paraffin or frozen immunohistochemistry. All steps were carried out at $4{ }^{\circ} \mathrm{C}$.

Fixation time for mouse samples from different developmental stages

\begin{tabular}{lll}
\hline Age & Time (h) for limb sample & Time (h) for spinal cord sample \\
\hline E10.5 & 1 & 1 \\
E12.5-13.5 & 2 & 2 \\
E14.5-16.5 & 4 & 3 \\
E17.5-18.5 & $12-16$ & 5
\end{tabular}

\subsubsection{Immunohistochemistry - Paraffin sections}

Hindlimb samples were dehydrated at $4^{\circ} \mathrm{C}$ every $4 \mathrm{~h}$ through ascending ethanol steps $(30 \%, 50 \%, 70 \%, 80 \%, 90 \%, 100 \%)$, then subsequently transferred to ethanol-xylene solution (1:1 ratio) and finally to xylene at room temperature (RT) every $4 \mathrm{~h}$. For paraffin infiltration, dehydrated tissues were incubated first with xylene-paraffin (1:1 ratio) for $4 \mathrm{~h}$ then incubated in paraffin three times for $1 \mathrm{~h}$ each time at $60{ }^{\circ} \mathrm{C}$ before routine embedding. Serial sections were cut on a Leica rotary microtome at 8-15 $\mu \mathrm{m}$ thickness, laid across a $42{ }^{\circ} \mathrm{C}$ water bath to remove creases and samples were picked up on Superfrost Plus microscope slides (Thermo Fischer Scientific GmbH). Sections were dried at $37{ }^{\circ} \mathrm{C}$ overnight before use or storage. During sectioning, a reference point was established to compare similar planes of distal hindlimb and forelimb between control and mutant mice. The reference point was defined as the first section containing both the fibula and tibia, or ulnar and radial bones. For all experiments, only sections with an equal distance from the reference point were compared. For 
immunohistochemistry, sections were deparaffinized and rehydrated by a descending series of ethanol $(100 \%, 90 \%, 80 \%, 70 \%, 50 \%, 30 \%), 5$ min per step. Sections were briefly rinsed with PBS for $5 \mathrm{~min}$ and were placed in a slide-holding chamber containing antigen retrieval solution diluted with double distilled water (1:50). The solution, together with the slides, were heated using a lab microwave for $5 \mathrm{~min}$ at 900 $\mathrm{W}$ until boiling point, and the power was reduced to $90 \mathrm{~W}$ for the next $15 \mathrm{~min}$. The slides were left to cool in the solution for a minimum of $20 \mathrm{~min}$. Thereafter, they were rinsed with PBS 3 times, 5 min each time before incubation with primary antibodies. The primary antibodies were diluted (in their respective working dilution) in immunostaining solution containing $1 \%$ Triton-X 1\% BSA in PBS and $500 \mu 1$ of solution was used per slide. Slides were then incubated at $4{ }^{\circ} \mathrm{C}$ overnight $(12-18 \mathrm{~h})$. Afterwards, they were washed with PBS for 4 times, 5 min each time at RT to rinse away unbound primary antibodies. Secondary antibodies were also diluted in immunostaining solution (1:1000) and $500 \mu \mathrm{l}$ of solution was used per slide. The slides were incubated at RT for $1 \mathrm{~h}$, then washed again with PBS for 4 times, 5 min each time to get rid of excess secondary antibodies, and rinsed with double distilled water briefly. To get rid of autofluorescence from sections, the slides were then incubated with cupric sulfate solution for $30 \mathrm{~min}$ in the dark at RT. The slides were washed once with double distilled water prior to mounting and visualization under a microscope.

\subsubsection{Immunohistochemistry - Frozen sections}

Spinal cord samples with vertebrae intact or whole embryos were equilibrated with $30 \%$ sucrose in PBS at $4{ }^{\circ} \mathrm{C}$ on a shaker. The tissues were then processed for routine embedding in plastic embedding molds containing OCT on dry ice. OCT blocks containing samples were stored at $-20{ }^{\circ} \mathrm{C}$ until use. Sections of $20-30 \mu \mathrm{m}$ thickness were cut on a Leica cryostat (CM1900, Leica Microsystems $\mathrm{GmbH})$ at $-20{ }^{\circ} \mathrm{C}$ and collected on Superfrost Plus microscope slides (Thermo Fischer Scientific GmbH). 
Slides were subsequently left to air dry at RT for approximately $3 \mathrm{~h}$ and stored at $-20^{\circ} \mathrm{C}$ thereafter or used immediately. For immunostaining, slides were rinsed four times with PBS for 15 min each time to remove residual OCT and to rehydrate samples. Slides were placed in a humidified chamber prior to primary antibody incubation. The primary antibodies were diluted (in their respective working dilution) in immunostaining solution and $500 \mu \mathrm{l}$ of solution was applied to each slide. Slides were then incubated at $4^{\circ} \mathrm{C}$ overnight (12-18 h). Afterwards, the slides were washed with PBS for 4 times, 5 min each time at RT to rinse away unbound primary antibodies. Secondary antibodies were also diluted in immunostaining solution and $500 \mu \mathrm{l}$ of solution was applied to each slide. The slides were incubated at RT for $1 \mathrm{~h}$, then washed again with PBS for 4 times, 5 min each time to get rid of excess secondary antibodies and finally mounted with cover slips using 50\% glycerol in PBS before visualization under a microscope.

For whole mount analyses, E18.5 hindlimb samples were fixed in 4\% PFA solution for 12-18 h, whereas E18.5 diaphragms were fixed for $2 \mathrm{~h}$. The samples were washed in PBS overnight, bleached with Dent's solution for $8 \mathrm{~h}$, then rehydrated overnight by using PBST. Following this, samples were incubated in primary antibody solution for 3 days, and then washed with PBST overnight before secondary antibody incubation for another 3 days. Finally, the samples were washed in PBST again overnight before dehydration by using a graded series of methanol solution up to $100 \%$. For better visualization, the samples were cleared with BABB solution before mounting between cover slips. All steps were carried out at $4{ }^{\circ} \mathrm{C}$. 


\subsection{Microarray experiments}

\subsubsection{Sample processing for microarray experiments}

Hindlimbs of embryonic mice (E15.5) were dissected with the skin and feet removed, and subsequently collected individually in microcentrifuge tubes containing $1 \mathrm{ml}$ TRIzol. Tail biopsies were also simultaneously obtained in separate tubes for genotyping afterwards. RNA (ribonucleic acid) of hindlimbs was extracted as described in Section 2.14.13., measured for concentration and quality control using NanoDrop (Perkin Elmer), before storing at $-80{ }^{\circ} \mathrm{C}$ until use for RNA amplification. Before use, RNA was left to thaw on ice for $10 \mathrm{~min}$ before proceeding with amplification steps. An input of $500 \mathrm{ng}$ of total RNA was used per sample in synthesizing labeled-aRNA (amplified RNA) from Illumina Total Prep RNA Amplification Kit. Amplification of RNA was done according to manufacturer's instructions to generate biotin-labeled aRNA. The biotinylated-aRNA was stored at $80{ }^{\circ} \mathrm{C}$ until further use in microarray hybridization experiments. A total of $1 \mu \mathrm{g}$ of biotinylated aRNA per sample was used for hybridization.

\subsubsection{Hybridization}

Illumina MouseRef-8 v2.0 expression BeadChips were used for microarray experiments. aRNA samples were sent to Max Planck Institute of Molecular Genetics (Berlin) to be hybridized on above-mentioned BeadChips as part of a collaboration. Microarray hybridization, imaging and data analysis were performed by Dr. Lars Wittler and Dr. Phillip Grote at the Max-Planck Institute of Molecular Genetics, Berlin. The output was an expression profile data (excel sheet format). 


\subsubsection{Pathway analysis}

The processed microarray data (Illumina IDs, along with the respective fold change values) were uploaded to Ingenuity Pathway Analysis Suite (Ingenuity Systems). Data were analyzed with the default settings except stringency and selection of filter parameters, which were set as 'high' and 'experimentally verified', respectively. The data were analyzed in the context of canonical pathways, molecular and physiological functions, diseases and networks. Later, the analyzed data were exported using the export function and was modified for representation using Photoshop CS5. Additionally, the data were also analyzed using the freeware DAVID (Database for Annotation, Visualization and Integrated Discovery) bioinformatics resource. The gene list (Illumina IDs) containing selected genes with fold-change of $\mathrm{p}<0.05$ were uploaded to DAVID and were analyzed using the default settings (Huang da et al., 2009b; Huang da et al., 2009a). Further, the functional annotation tool enabled selected genes to be mapped to KEGG (Kyoto Encyclopedia of Genes and Genomes) pathways (Kanehisa and Goto, 2000). For Panther gene expression analysis, gene lists containing significantly up- or downregulated genes were uploaded to the workspace and the species genome (Mus musculus) was selected. The data were analyzed in the context of molecular and biological processes (Mi et al., 2010; Mi et al., 2012).

\subsection{RNA sequencing experiments}

\subsubsection{Sample processing for RNA-seq experiments}

Hindlimb samples of E14.5 embryonic mouse hindlimbs were collected as described for microarray experiments (Section 2.6) and were sent to a transcriptomic facility at the Göttingen Center for Molecular Biosciences Institute (Göttingen, Germany) for RNA isolation and further processing (RNA sequencing, imaging and data analysis). The concentrated RNA was checked for quality and quantity on a Pico-chip (Agilent 
RNA 6000) using the Bioanalyzer 2000 (Agilent Technologies). RNA sequencing output was an expression profile data (excel sheet format).

\subsubsection{Pathway analysis}

Candidate genes were selected and analyzed with criteria and tools described for microarray pathway analysis.

\subsection{Quantitative reverse-transcription PCR (qPCR)}

RNA as obtained as described in Section 2.14.13. cDNA (complementary deoxyribonucleic acid) was reversely transcribed from RNA of mouse hindlimbs using Roche cDNA synthesis kit according to manufacturer's instructions $(1 \mu \mathrm{g}$ of input RNA was used per sample). Primer design for genes of interest was performed by obtaining the accession number of the gene of interest (longest transcript variant was chosen) from http://www.pubmed.com and inputting its accession number on Roche website for real-time $\mathrm{qPCR}$ assay design http://www.roche-appliedscience.com/sis/rtpcr/upl/ezhome.html. Optimal left and right primers with sequence specific for gene of interest was obtained along with a compatible Taqman probe number. qPCR experiments were performed on a Lightcycler 480 machine (Roche Diagnostics $\mathrm{GmbH}$ ) with 55 cycles per run, and with an in-run efficiency factor. Each reaction was perfomed in duplicate and normalized to levels of loading controls Alpha-tubulin (TUBA3), or Hypoxanthine-Guanin-Phosphoribosyltransferase (HPRT), which tested to have no differential expression between control and mutant mice. Values obtained from mutant muscles were normalized to control levels (\%). 


\subsection{Electron microscopy experiments}

\subsubsection{High pressure freezing and Freeze substitution}

Complete limbs of E15.5 embryos were fixed in 2.5\% glutaraldehyde in PBS for $3 \mathrm{~h}$ on ice and briefly washed in PBS. Muscles were gently teased apart and frozen in $200 \mu \mathrm{m}$ deep aluminium platelets (Microscopy Services, Flintbek) using 1-hexadecane as a freezing medium using a BalTec HPM 10. Freeze substitution was carried out in a Leica EM AFS at $-90^{\circ} \mathrm{C}$ for $24 \mathrm{~h}$ in $0.1 \%$ tannic acid, followed by $2 \% \mathrm{OsO}_{4}$ for $22 \mathrm{~h}$ (each $\mathrm{w} / \mathrm{v}$ in dry acetone) with slowly increasing temperature, according to (Rostaing et al., 2006). All electron microscopy steps were performed by Dr. Christoph Wrede as part of a collaboration.

\subsubsection{Imaging}

$50 \mathrm{~nm}$ longitudinal sections were cut using a Leica UC6 ultramicrotome. Sections were transferred on Formvar-coated copper single slot-grids. Grids were placed for $45 \mathrm{~min}$ on drops of $4 \%(\mathrm{w} / \mathrm{v})$ uranyl acetate (diluted in distilled water), followed by distilled water. Air-dried grids were incubated on drops of lead citrate (Reynolds, 1963) for 2 $\min$ in a $\mathrm{CO}_{2}$-free atmosphere, and rinsed in distilled water. Specimens were examined in a Zeiss EM 902A, equipped with a $1024 \times 1024$ CCD detector (Proscan CCD HSS 512/1024; Proscan Electronic Systems, Scheuring, Germany).

\subsection{Pulse-chase experiments}

Click-iT EdU Fluor 555 Imaging Kit was obtained from Roche diagnostics $\mathrm{GmbH}$ and EdU was dissolved in PBS at a stock concentration of $10 \mathrm{mg} / \mathrm{ml}$. Pregnant mice were injected with EdU intraperitoneally at a concentration of $50 \mathrm{mg} \mathrm{kg}^{-1}$ body weight. Injection was performed at 14.5 and $15.5 \mathrm{dpc}$ consecutively to cover onset of secondary 
myogenesis. Embryos were retrieved at E18.5 and were processed in the same way as described in Section 2.5.1. EdU detection was done according to manufacturer's instructions. Following washing steps, immunohistochemistry was performed on the slides. The slides were then counterstained with DAPI before mounting.

\subsection{Imaging}

Fluorescence images (transverse sections and whole mounts) were visualized and taken using a Leica SP2 TCS/MP confocal/two-photon microscope (Leica Microsystem $\mathrm{GmBH}$ ) with $488 \mathrm{~nm}, 543 \mathrm{~nm}$ and $633 \mathrm{~nm}$ laser lines. Pictures were taken at resolutions of either 1024 x 1024 or 2048 x 2048 pixels. The images were processed using Adobe Photoshop CS5.

\subsection{Quantification}

Adobe Photoshop CS5 or Image J was used for all quantification done. For all data sets, a minimum of three animals from each genotype $(n=3)$ per stage was used for quantification, unless otherwise stated.

For muscle mass quantification, five planes of increasing distance (two sections were randomly chosen from each plane) from the reference point were selected per animal. An outline was made at the border of each muscle contained within the sections and the pixels occupied by the muscles were summed up. Values obtained from mutant mice were normalized to control mice.

For cleaved caspase-3 quantification, four sections equidistant from the reference point were randomly chosen per animal. Only cleaved caspase-3 positive cells that were found within the boundaries of muscles were counted to obtain an absolute number of cleaved caspase $3^{+}$cells. 
Muscle fiber density and cross-sectional area was quantified by randomly placing a box of defined area $(60 \times 60$ pixels) within the soleus (S) and tibialis anterior (TA) muscle. The number of muscle fibers found within this box was counted. The crosssectional area (CSA) of these muscle fibers was also quantified by outlining the perimeter of each individual fiber and obtaining the pixels contained within the area. Values obtained from mutant mice were normalized to control mice.

For counts of $\mathrm{Pax}^{+}, \mathrm{EdU}^{+}$or slow $\mathrm{MyHC}^{+}$myofibers to total laminin ${ }^{+}$myofibers on transverse TA and S muscles control and mutant limbs, three sections of each muscle equidistant from the reference point were selected per animal. Counts of nine sections were averaged for three individuals of each genotype. The cell counter function in Image J (http://rsb.info.nih.gov/ij) was used to obtain the proportion of Pax $7^{+}$cells:total laminin ${ }^{+}$cells, slow $\mathrm{MyHC}^{+}$fibers:total laminin ${ }^{+}$fibers or $\mathrm{EdU}^{+}$cells:total laminin ${ }^{+}$cells on E18.5 hindlimb transverse sections. Images for quantification were taken at $63 \mathrm{x}$ magnification.

\subsection{Statistical analysis}

Prism 5.0 software was used for all statistical analysis unless otherwise stated. Statistical comparisons between control and mutant mice were established using a two-tailed Student's $t$ test with unequal variance, where applicable and unless otherwise stated. Data were represented as the mean \pm standard error of the mean (SEM).

\subsection{Molecular biology}

The following methods described were performed according to 'Molecular cloning: a laboratory manual (Sambrook and Russell, 2001) unless otherwise stated. 


\subsubsection{Polymerase chain reaction}

The amplification of DNA fragments was performed using either Platinum Taq DNA Polymerase High Fidelity, Phusion High Fidelity DNA Polymerase or Dream Taq DNA Polymerase on the Mastercycler epGradient S (Eppendorf AG). The reaction mixture and the PCR conditions used were set up according to the manufacturer's protocols. Amplified DNA fragments were detected via agarose gel electrophoresis. DNA fragments with correct band length with reference to a loading ladder were isolated and extracted using QIAquick Gel Extraction Kit.

\subsubsection{DNA restriction enzyme digestion}

DNA digestion (vectors or inserts) was performed to yield 3' overhangs or blunt ends according to appropriate restriction enzymes used. The DNA was incubated with the enzymes and the reaction buffers at optimal reaction temperatures as recommended by the manufacturer for at least $3 \mathrm{~h}, 16-18 \mathrm{~h}$ if performing double digestion. Double digestions were carried out with reaction buffers that will enable maximal enzyme activity for both endonucleases.

\subsubsection{Alkaline phosphatase treatment}

To prevent self-ligation, the vector DNA was dephosphorylated by adding Shrimp Alkaline Phosphatase to the completed restriction digest mixture of the vector DNA and incubated for $30 \mathrm{~min}$ at $37^{\circ} \mathrm{C}$.

\subsubsection{Agarose gel electrophoresis}

The separation of DNA fragments was performed by agarose gel electrophoresis with $1 \%[\mathrm{w} / \mathrm{v}]$ agarose dissolved in TAE buffer by heating, followed by addition of 
$0.05 \mu \mathrm{l} / \mathrm{ml}$ of ethidium bromide solution and transferring into a gel cast. Combs of suitable sizes were placed in the warm agarose solution, depending on the number of wells needed. After solidifying, the gel was immersed into a gel tank containing TAE buffer. DNA samples were mixed with 5x DNA loading dye and loaded into the wells. An electrical current was applied until a definite separation of the DNA fragments was achieved.

\subsubsection{DNA purification from agarose gels}

After agarose gel electrophoresis, gels were removed from the gel tanks and DNA bands were visualized under UV light illumination. DNA fragments with correct band length were extracted from the agarose gel and later purified by using the QIAquick Gel Extraction Kit.

\subsubsection{DNA ligation}

Digested vector and insert DNA concentration were determined and the ligation mixture of DNA fragments was prepared with a molar vector to insert concentration ratio of 1:9. Thereafter, the reaction mix was incubated with T4 DNA Ligase and the provided buffer at $22^{\circ} \mathrm{C}$ for a minimum of $6 \mathrm{~h}$ or with the DNA Ligation Kit (Takara Bio Inc) at $16^{\circ} \mathrm{C}$ for at least $2 \mathrm{~h}$. For PCR products with low concentrations, the TOPO TA Cloning Kit (Life Technologies $\mathrm{GmbH}$ ) was used according to the manufacturer's instructions.

\subsubsection{Plasmid preparation}

Bacterial cells were grown from single colonies on agar plates or bacterial stocks. A toothpick was used to pick a single colony of bacteria and was then cultured in $5 \mathrm{ml}$ 
LB medium containing ampicillin at $37{ }^{\circ} \mathrm{C}$ overnight with $180 \mathrm{rpm}$ shaking. Smallscale plasmid isolation was performed using the QIAprep Spin Miniprep Kit (Qiagen $\mathrm{GmbH}$ ) following manufacturer's instructions. Extracted DNA was eluted in $30 \mu \mathrm{l}$ of elution buffer. Positive clones were verified via restriction digest and sequencing. Large-scale plasmid isolation (for in ovo electroporation) was performed by growing $100 \mu \mathrm{l}$ of E.coli culture from glycerol stock in $200 \mathrm{ml} \mathrm{LB}$ medium with ampicillin overnight at $37^{\circ} \mathrm{C}$ with $180 \mathrm{rpm}$ shaking. Plasmid DNA was extracted using the QIAGEN Plasmid Maxi Kit according to the instruction manual and eluted in an appropriate volume of buffer.

\subsubsection{DNA and RNA concentration measurements}

Concentration and quality of nuclei acids were measured using Nanodrop ND-1000 UV-Vis spectrophotometer (Peqlab Biotechnologies $\mathrm{GmbH}$ ) based on UV light absorption of nuclei acids at specific wavelengths.

\subsubsection{Sequencing}

DNA sequencing was commercially carried out by Qiagen genomic services (Qiagen $\mathrm{GmbH}$ ). DNA samples were submitted at a concentration of $500 \mathrm{ng}$ per reaction for sequencing. Only plasmids with entirely precise sequences were selected for use.

\subsubsection{Preparation of chemically competent E.coli}

Chemically competent E.coli cells of the One Shot TOP10 strain (Life Technologies $\mathrm{GmbH}$ ) were used for all molecular cloning experiments. The preparation was performed according to Inoue et al. (Inoue et al., 1990) and stored at $-80^{\circ} \mathrm{C}$ until use. 


\subsubsection{Transformation}

Chemically competent E.coli cells were transformed by adding $100 \mu \mathrm{l}$ of the soluble cell suspension to a maximum amount of $10 \mu 1$ ligated DNA ligation mixture. The cells were incubated $30 \mathrm{~min}$ on ice, heat shocked at $42^{\circ} \mathrm{C}$ for $1 \mathrm{~min}$ and chilled on ice afterwards again for $1 \mathrm{~min}$. E.coli were grown in $1 \mathrm{ml}$ of antibiotic-free LB medium at $37^{\circ} \mathrm{C}$ for $1 \mathrm{~h}$ on a shaking $(850 \mathrm{rpm}$ ) heatblock and centrifuged for $2 \mathrm{~min}$ at $3000 \mathrm{rpm}$ before they are subsequently plated on an agar plate containing ampicillin. The plates were incubated overnight at $37^{\circ} \mathrm{C}$ for $16-18 \mathrm{~h}$.

\subsubsection{Preservation of E.coli}

The preservation of the E.coli clones was achieved by mixing $500 \mu \mathrm{l}$ of soluble E.coli culture with $500 \mu 1100 \%$ glycerol in a microcentrifuge tube, vortexing shortly and storing the mixture at $-80^{\circ} \mathrm{C}$ until further use.

\subsubsection{RNA extraction}

Total RNA was obtained from muscle and spinal cord samples of E13.5, E15.5 and E18.5 mouse embryos. The tissue was collected in a microcentrifuge tube and homogenized in $1 \mathrm{ml}$ TRIzol reagent per $100 \mathrm{mg}$ of tissue using a dounce homogenizer followed by incubation at RT for $5 \mathrm{~min}$. The samples were incubated with $0.2 \mathrm{ml}$ chloroform per $1 \mathrm{ml}$ TRIzol for $3 \mathrm{~min}$ and centrifuged for $15 \mathrm{~min}$ at $4^{\circ} \mathrm{C}$ at $12000 \mathrm{rpm}$. The aqueous phase containing the RNA was carefully removed so as to not disturb the interphase, transferred into a new microcentrifuge tube and incubated with $0.5 \mathrm{ml}$ isopropanol per $1 \mathrm{ml}$ of TRIzol and $3 \mu \mathrm{l}$ of polyacrylamide for $10 \mathrm{~min}$ at RT. After centrifugation for $15 \mathrm{~min}$ at $4^{\circ} \mathrm{C}$ at $12000 \mathrm{rpm}$, the supernatant was discarded and the RNA pellet was washed with $1 \mathrm{ml}$ cold $75 \%$ DEPC-ethanol. The samples were centrifuged again for $5 \mathrm{~min}, 4^{\circ} \mathrm{C}$ at $7500 \mathrm{rpm}$ and subsequently air-dried and eluted in 
DEPC- $\mathrm{H}_{2} \mathrm{O}$. Incubation of the eluted pellet at $50^{\circ} \mathrm{C}$ for 10 min was occasionally performed to aid in dissolving the RNA pellet.

\subsubsection{4.cDNA synthesis}

The cDNA used for gene amplification and in situ probe generation was synthesized using the cDNA synthesis Kit (Roche Diagnostics $\mathrm{GmbH}$ ) or the PrimeScript First Strand cDNA Synthesis Kit (Takara Bio Inc).

\subsection{P2TK-MLC-V5-MCS-2A-eGFP plasmid construction}

This plasmid was designed to study gain-of-function of candidate genes obtained from microarray data specifically in muscles of chick embryos, when combined with Tol2 transposon-based constructs which will facilitate genomic integration upon in ovo somite electroporation (Kawakami and Shima, 1999; Sato et al., 2007; Wang et al., 2011a). This plasmid was modified from the original vector $p T 2 K-M L C-E G F P-F$ (described in Wang et al., 2011), a kind gift from D. Duprez. The $p T 2 K-M L C-E G F P-F$ plasmid contains the enhancer and promoter region of the mouse myosin light chain $1 F / 3 F$ locus. The promoter and enhancer region was excised from the $p T 2 K-M L C$ EGFP-F vector using XhoI and NcoI restriction enzymes and inserted into the $p 2 T K-$ $C M V-2 A-e G F P$ vector, a vector that was constructed in the lab, replacing the CMV enhancer that was used to drive ubiquitous expression of transgenes. From this, the p2TK-MLC-2A-eGFP vector was generated. To create insertion sites for genes of interest, V5-tag and multiple cloning site (MCS) sequences with NcoI and EcoRI as restriction sites were designed as long oligonucleotide primers (5'CATGTTGGGT AAGCCTATCC CTAACCCTCT CCTCGGTCTC GATTCTACGC CATGGATGCA GGAATTCAA3'; 3'CATGTTGAAT TCCTGCATCC ATGGCGTAGA ATCGAGACCG AGGAGAGGGT TAGGGATAGG CTTACCCAA5'). 
The oligonucleotides were annealed together and later digested with NcoI and EcoRI. T4 Polynucleotide Kinase was added to the completely digested annealed oligonucleotides and incubated for $1 \mathrm{~h}$ to allow for the addition of phosphates to $5^{\prime}$ hydroxyl terminus of oligonucleotides before sticky-ended ligation into the $p 2 T K$ $M L C-2 A-e G F P$ vector, to yield the final $p 2 T K-M L C-V 5-M C S-2 A-e G F P$ vector. In summary, the $p 2$ TK-MLC-V5-MCS-2A-eGFP vector consists of six essential parts: 1) the $M L C 1 F / 3 F$ enhancer and promoter region to specifically drive transgene expression in muscle cells, 2) V5 epitope tag sequence for protein detection, 3) gene insertion sites, 4) followed by sequence for $2 \mathrm{~A}$ peptide bridge which is 5) upstream reporter gene $e G F P$ (enhanced green fluorescent protein) and 6) Tol2 sites which flanked the expression cassette for transposase-mediated stable genomic integration. The $p$ CAGGS-T2TP was co-electroporated for a stable genetic integration of transgenes in the transfected muscle cells.

\subsection{In ovo electroporation}

In ovo electroporation of DNA constructs was performed between HamburgerHamilton $(\mathrm{HH})$ stages $15-18$ by using the ECM 830 electroporation system (BTX Instrument Division, Harvard Apparatus Inc) with the following settings: 5 square wave pulses (interval of $200 \mathrm{msec}$ ) of $28 \mathrm{~V}$ for $55 \mathrm{~ms}$ in the LV 99ms/500 V modus. Prior to electroporation, a small puncture was made into the eggshell and a syringe was inserted into the egg to remove $5 \mathrm{ml}$ of albumen. Thereafter, an oval window was made from the small puncture to visualize the embryo. The egg was placed on a homemade egg holder. Needles for injections were pulled from glass capillaries and were filled with plasmid mixed with fast green dye (10\% of plasmid volume). All plasmids were injected at final concentrations of $3.0 \mu \mathrm{g} / \mu \mathrm{l}$ in TE buffer. The molar ratio for expression vector and transposase vector was 2:1. For somite injection, the needle was pushed through somite number 25-30 and the p2TK-MLC-V5-MCS-2A- 
eGFP and pCAGGS-T2TP plasmids were injected into the somite coeloem by mouth pressure whilst slowly retracting the needle. In order to genetically ablate motor neurons in the chick embryo, neural tube electroporation was performed. The needle containing PGKdtapa vector was inserted at a $45^{\circ}$ angle into the neural tube lumen. Electroporation was done immediately after injection by applying $150 \mu \mathrm{l}$ of Lebowitz15 medium onto the amniotic sac, and then placing the embryo between two platinum electrodes (for somite electroporation, the side of injected somites was always positioned next to the cathode electrode). The oval window was sealed with cellophane tape and the egg returned to the incubator until the desired stage was reached. 


\section{Results}

\subsection{Creating aneural muscles by genetic ablation of motor neurons}

To address the impacts of denervation on embryonic myogenesis, genetic tools more powerful than manual dissection or pharmalogical agents can be used to generate aneural muscles. Advancement in technology has allowed for the generation of transgenic mouse lines, one of them being $R 26^{f x D T A}$, that are used for specifically ablating cells of any lineage (Ivanova et al., 2005). Diphtheria toxin (DT), secreted by Cornynebacterium diphtheriae is made up of two subunits: DTA, and DTB. Target cell death is brought about by the DTA subunit of the toxin. This subunit is responsible for the inactivation of elongation factor 2, resulting in the cessation of protein synthesis (Maxwell et al., 1986; Palmiter et al., 1987; Breitman et al., 1990). Using a Cre/loxP approach, two different mouse lines with conditional motor neuron ablation were generated.

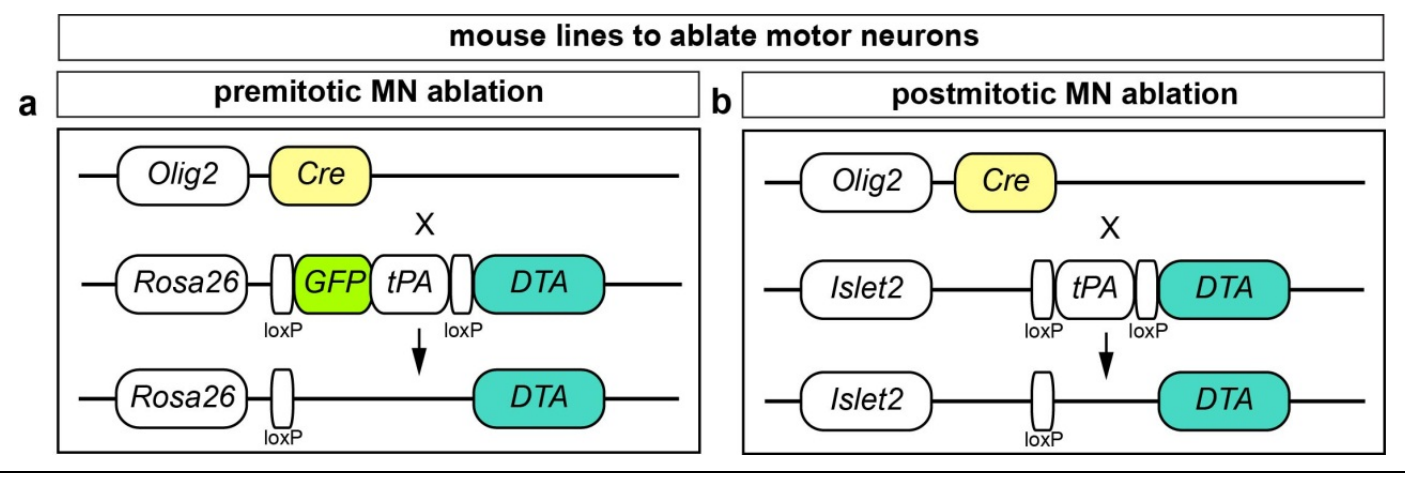

Figure 3.1: Strategy to genetically ablate motor neurons in mouse.

(a) To generate mice with premitotic motor neuron ablation, Olig2 ${ }^{\text {Cre }}$ mouse line was crossed with the $R 26^{f x D T A}$ mouse line to generate $R 26^{f x D T A} x$ Olig2 ${ }^{\text {Cre }}$ mice. Cre-mediated excision of floxed region will lead to DTA expression in motor neuron progenitors. (b) To generate a mouse line with postmitotic motor neuron ablation, Olig2 ${ }^{\mathrm{Cre}}$ mouse line was crossed with the Isl2 ${ }^{\text {x DTA }}$ mouse line to generate Isl2 fxDTA $x$ Olig2 ${ }^{\mathrm{Cre}}$ mice. Cre-mediated recombination will result in postmitotic motor neuron cell death. DTA, diphtheria toxin subunit A coding sequence; $t P A$, stop sequence; GFP, green fluorescent protein coding sequence. 
First, a mouse line engineered to drive Cre-mediated recombination in Olig2expressing cells was bred to $R 26^{f x D T A}$ to generate $R 26^{f x D T A} x O l i g 2^{C r e}$ mice. Since $R O S A 26$ is a ubiquitous promoter and Olig2 is expressed specifically in motor neuron progenitors, Cre-mediated excision of a "floxed" genomic region flanked by two loxPsites region will facilitate DTA expression and ablation of motor neuron progenitors (Figure $3.1 \mathrm{a}$ ). Olig2 is known to be critical in the generation of oligodendrocytes at later stages of development (Nabeshima et al., 1993). To address if possible phenotypes are associated with oligodendrocyte ablation, a second mouse line was generated by crossing Olig2 ${ }^{C r e}$ mice with Isl2 ${ }^{f x D T A}$ mice to generate Isl2 ${ }^{f x D T A} x O \operatorname{Oig} 2^{\mathrm{Cre}}$ mice (Figure $3.1 \mathrm{~b}$ ). Isl2 is one of the first homeodomain proteins to be expressed in newly postmitotic motor neurons (Jessell, 2000; Dasen and Jessell, 2009). Therefore, in Isl2 $2^{\text {fXTA }} x$ Olig2 $2^{\mathrm{Cre}}$ embryos, Cre-mediated recombination will specifically eliminate postmitotic motor neurons, while in $\mathrm{R}_{2} 6^{f \times D T A} x \mathrm{Olig} 2^{\mathrm{Cre}}$ mice generation of postmitotic motor neurons will be suppressed from the outset by ablating their progenitors.

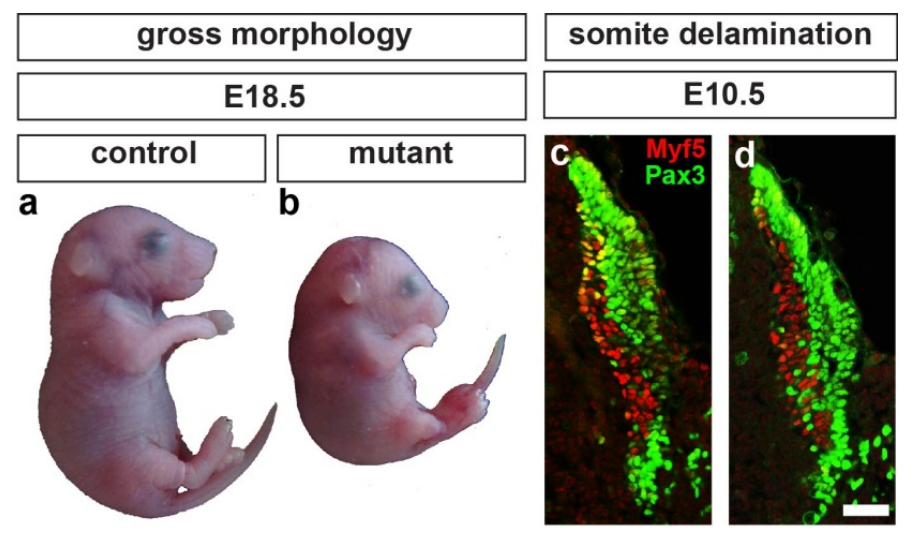

Figure 3.2: Motor neuron ablation does not affect somite delamination.

(a-b) E18.5 (b) R26fxDTA $x$ Olig2 ${ }^{\text {Cre }}$ mutant mice devoid of motor neurons exhibit normal gross morphology, perinatal lethality and remained in fetal position as compared to (a) controls. (c-d) Somite formation and further delamination is unaffected after motor neuron ablation. Muscle precursors $\left(\mathrm{Myf5}^{+}\right.$and $\left.\mathrm{Pax3}^{+}\right)$are formed in both (c) control and (d) mutant mice. E, embryonic day. Myf5, myotome marker; Pax3, dermomyotome marker. Scale bar: $50 \mu \mathrm{m}$. 
Both Isl2 fxDTA $x$ Olig $2^{\mathrm{Cre}}$ and $\mathrm{R} 26^{\mathrm{fxDTA}} \mathrm{xOlig} 2^{\mathrm{Cre}}$ mice exhibit perinatal lethality, due to respiratory failure caused by the lack of innervation of the diaphragm. Phenotypic analysis performed revealed no abnormal gross morphology, but a kyphosis-like position of mutant mice compared to controls, after wholesale removal of motor neurons (Figure $3.2 \mathrm{a}-\mathrm{b}$ ). At the same time, somite formation and delamination of myoblasts was unaffected by motor neuron ablation. This is evidenced by the observation that $\mathrm{Pax3}^{+}$and $\mathrm{Myf}^{+}$muscle precursors were formed in both control and mutant mice (Figure $3.2 \mathrm{c}-\mathrm{d}$ ).

Postmitotic motor neurons expressing vesicular acetylcholine transferase-positive (vAChT) are generated already by embryonic day (E) 9.5 (earliest time point investigated) in the ventral part of the spinal cord, and can be observed to project their axons into the periphery by E10.5 (Figure 3.3 a). At the same time, $\mathrm{vAChT}^{+}$motor neurons were not detected in the spinal cord of both Isl2 ${ }^{f x D T A} x O \operatorname{Oig} 2^{\text {Cre }}$ and $R 26^{f x D T A} x O \operatorname{Oig} 2^{\text {Cre }}$ mouse lines, indicating that they were either not generated or ablated soon after being generated (Figure $3.3 \mathrm{~b}-\mathrm{c}$ ). Accordingly, motor axon projections were absent in both mutant mice. To verify that other cell types were not ablated, I stained transverse spinal cord sections of E10.5 mice with Isl1/2, another generic motor neuron marker which also labels sensory neurons located in the dorsal root ganglion (DRG) (Figure $3.3 \mathrm{~d}$-f). Nuclei staining located in ventral part of the spinal cord was completely absent from $\mathrm{R}_{2} 6^{f x D T A} x \mathrm{Olig} 2^{\text {Cre }}$ mice (Figure $3.3 \mathrm{e}$ ). A faint staining of several cells was observed seen in the ventral spinal cord of Isl2 ${ }^{f x D T A} x O \operatorname{Oig} 2^{\mathrm{Cre}}$ mice (Figure 3.3 f). These were hypothesized to be newly formed postmitotic motor neurons which have yet to accumulate a threshold level of Isl2 protein to be sufficient to drive expression of the DTA allele and will be ablated after this threshold is reached. Furthermore, to rule out that the absence of motor neurons observed at E10.5 was because of delayed generation, spinal cords of E18.5 mice were analyzed (Figure $3.3 \mathrm{~g}$ i). 


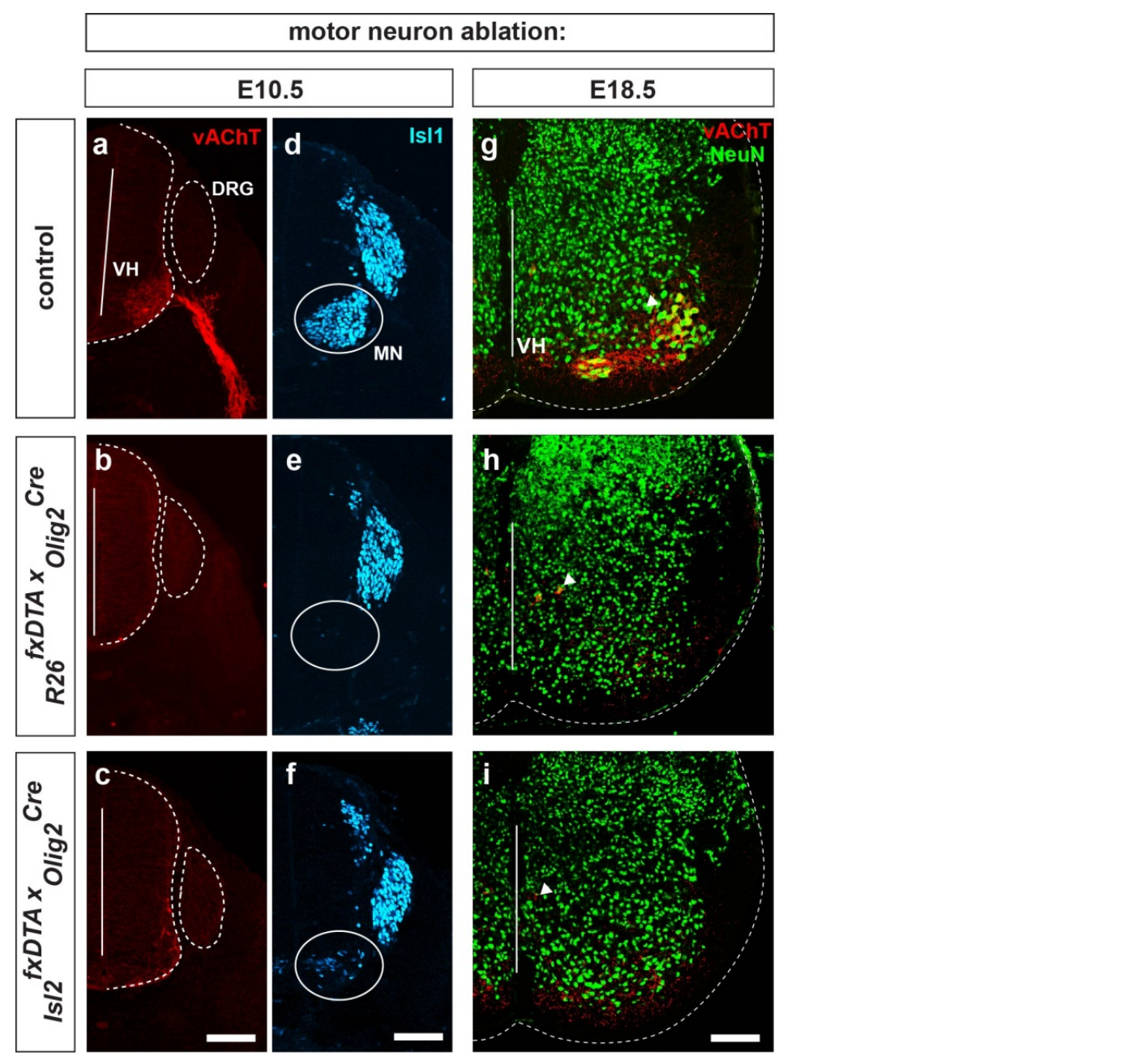

Figure 3.3: Specificity of motor neuron ablation in mutant mouse lines.

(a-c) At E10.5, motor neurons were formed in (a) control mice but were absent from the $\mathrm{VH}$ of the spinal cord of (b) R2 $6^{f x D T A} x$ Olig2 ${ }^{\text {Cre }}$ and (c) Isl2 ${ }^{f x D T A} x O l i g 2^{C r e}$ mice. (b-c) Peripheral motor axon projections were also absent in mutant mice. (d-f) $\mathrm{Isl}^{1 / 2^{+}}$staining was present in the DRG and motor neurons of (d) control mice, but was absent from the $\mathrm{VH}$ of the spinal cord of (e) $R 26^{f x D T A} x \mathrm{Olig} 2^{\mathrm{Cre} e}$ mice. (f) Isl $1 / 2$ protein is expressed at very low levels in a few cells in Isl2 fxDTA $x$ Olig2 Cre spinal cord. (g-i) At E18.5, vAChT $^{+}$ motor neurons located in the $\mathrm{VH}$ of the spinal cord of (g) control mice were not detected in the spinal cord of (h) R26fxDTAxOlig2 Cre and (i) Isl2 fxDTA $x$ Olig2 ${ }^{\mathrm{Cre}}$ mice. However, $\mathrm{VAChT}^{+}$cholinergic interneurons located near the central canal were still present in the spinal cord of both mutant mouse lines. VH, ventral horn; DRG, dorsal root ganglion, MN, motor neurons. vAChT, cholinergic neuron marker; Isl12/2, pan motor neuron and DRG marker; NeuN, pan neuronal marker. Arrows indicate cholinergic interneurons, bold lines mark midline of spinal cords and dotted lines demarcate border of spinal cords and DRG. Circles outline where motor neurons are expected to be. All scale bars: $100 \mu \mathrm{m}$. 
iAt E18.5, both Isl2 ${ }^{f x D T A} x \mathrm{Olig} 2^{\mathrm{Cre}}$ and $\mathrm{R}^{\mathrm{f} 6^{f x D T A}} \mathrm{XOlig} 2^{\mathrm{Cre}}$ exhibited virtually complete absence of motor neurons (large $\mathrm{NeuN}^{+}$and $\mathrm{vAChT}^{+}$cells) while cholinergic V0c interneurons adjacent to the central canal remained unaffected (Figure $3.3 \mathrm{~h}-\mathrm{i}$ ), thereby demonstrating the specificity of the two mouse lines in ablating motor neurons and producing embryos with aneural skeletal muscle.

\subsection{Analysis aneural skeletal muscles}

\subsubsection{Removal of motor neurons results in skeletal muscle hypoplasia}

It has long been assumed that muscles depend on motor neurons for their normal development (Hunt, 1932; Shellswell, 1977; Butler et al., 1982; McLennan, 1994). However, the exact role of innervation for embryonic myogenesis remains controversial (Butler et al., 1982; Ross et al., 1987; Condon et al., 1990; Fredette and Landmesser, 1991a; Hughes and Ontell, 1992; Wilson and Harris, 1993), while the underlying mechanisms remain elusive. Many uncertainties seem to be due to technical limitations of previous studies addressing the role of innervation for embryonic myogenesis, which exclusively relied on classical embryological methods, such as manual surgical removal of neural tube segments. To sidestep these limitations, I therefore investigated muscle development in completely aneural $R 26^{f x D T A} x O \operatorname{Oig} 2^{\text {Cre }}$ embryos. I have analyzed fore- and hindlimb transverse sections of mutant mice at late-embronic stages (E18.5) and found a drastic reduction in muscle mass (determined by staining with MF20, a pan myofiber marker) in both $R 26^{f x D T A} x O l i g 2^{C r e}$ and Isl2 ${ }^{f x D T A} x O l i g 2^{C r e}$ mouse lines (Figure $\left.3.4 \mathrm{a}-\mathrm{f}\right)$. The reduction in muscle mass was not limited to only the limbs, but muscles in other parts of the body such as the diaphragm and intercostal muscles (data not shown). This indicates that the lack of motor neurons resulted in systemic skeletal muscle hypoplasia. Additionally, some groups of muscles appeared to be more severely affected compared to others. Cross examination of hindlimb of mutant mice revealed that 
while muscles like tibialis anterior (TA), extensor digitorum longus (EDL) and soleus (S) were reduced, other muscle groups such as gastrocnemius ( $\mathrm{Gm}$ and $\mathrm{Gl}$ ) were almost completely abolished (Figure 3.4 b-c).

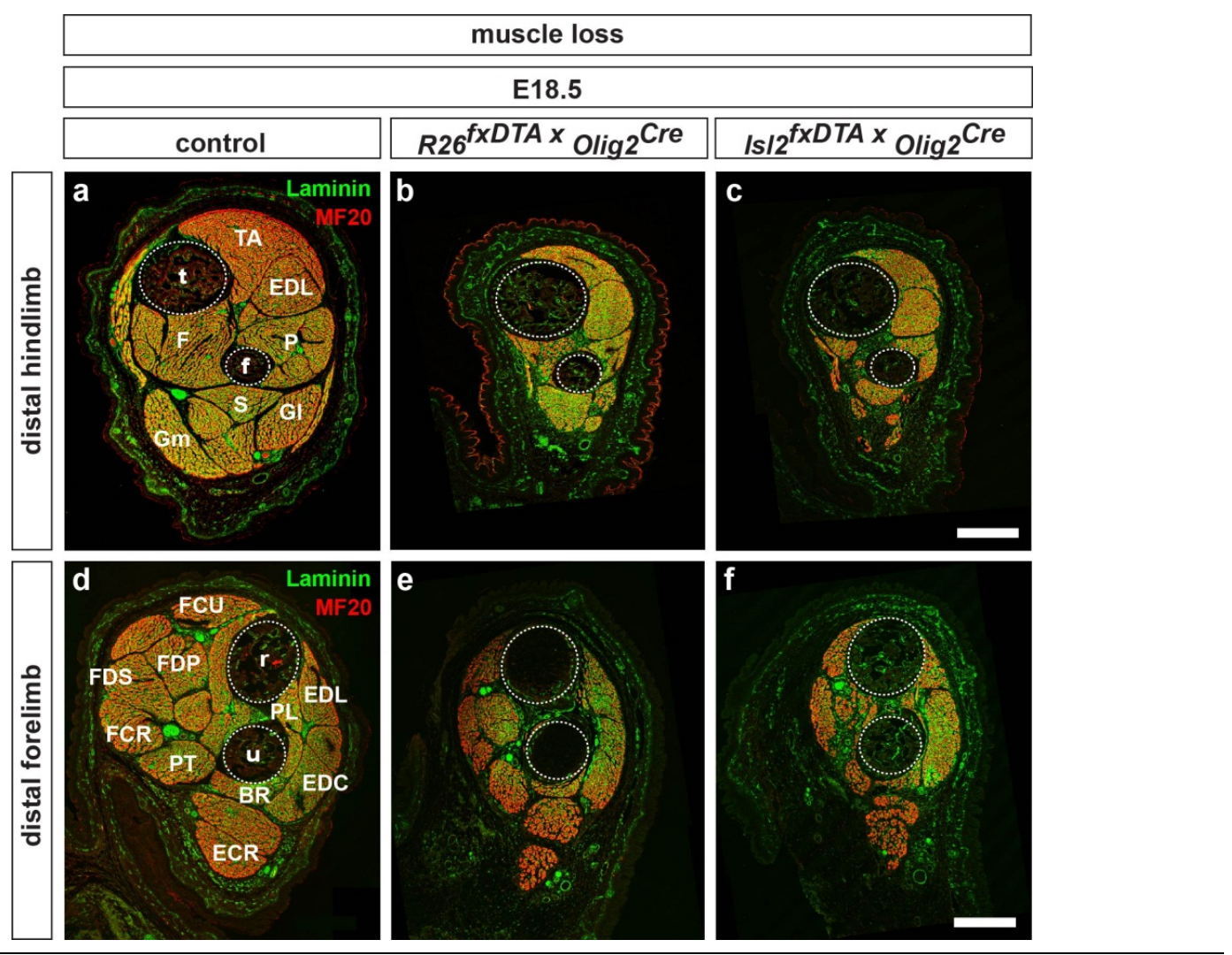

Figure 3.4: Removal of motor neurons results in general skeletal muscle hypoplasia.

(a-c) Transverse distal hindlimb sections of E18.5 (a) control, (b) R26 fxDTAxOlig2cre and (c) Isl2 ${ }^{x D T A} x \mathrm{Olig} 2 \mathrm{Cre}$ mice. Muscle mass were reduced without motor innervation, but some muscles were more affected than others. Gm and Gl muscles disappeared almost completely, while other muscles such as TA and S muscles were significantly reduced. (d-f) Similarly, muscles of the distal forelimb were also severely affected by the absence of motor neurons. (d) E18.5 control distal forelimbs contained many groups of muscles that were absent in the forelimb of (e) R26fxDTAxOlig2 $2^{\text {Cre }}$ and (f) Isl2 $2^{f x D T A} x$ Olig2 ${ }^{C r e}$ mice. E, embryonic day; $\mathrm{t}$, tibia bone; $\mathrm{f}$, fibula bone; $\mathrm{r}$; radial bone; $\mathrm{u}$, ulnar bone; TA, tibialis anterior; $\mathrm{EDL}$, extensor digitorum longus; $\mathrm{P}$, peroneus muscle group; $\mathrm{F}$, fibula muscle group; $\mathrm{S}$, soleus; Gm, medial gastrocnemius; Gl, lateral gastrocnemius; BR, brachioradials; ECR, extensor carpi radialis brevis; EDC, extensor digitorum commusis; EDL, extensor digitorum longus; FCR, flexor carpi radialis; FCU, flexor carpi ulnaris; FDP, flexor digitorum profundus caput fadiale; PL, pollicis longus; PT, pronator teres. Laminin, basal lamina marker; MF20, pan myosin marker. Dotted lines indicate border of bones with distal forelimb and hindlimb transverse sections. All scale bars: $250 \mu \mathrm{m}$. 
To determine the degree of skeletal hypoplasia, the remaining muscle mass was quantified in both fore- and hindlimb sections throughout different developmental stages (Figure 3.5 a-d). Examination of $\mathrm{R}^{66^{f x D T A}} x \mathrm{Olig} 2^{\mathrm{Cre}}$ and Isl2 ${ }^{f x D T A} x \mathrm{Olig} 2^{\mathrm{Cre}}$ mice revealed that the onset of muscle loss begins at E14.5, with an initial reduction of about $17 \%$ and $12 \%$ when normalized to control mice, respectively (Figure 3.5 a and c). Muscle loss became more pronounced during subsequent stages of embryogenesis. At E15.5, muscle mass of mutant mice was reduced by $43 \%$. At E18.5, which was the latest stage that could be analyzed due to the perinatal lethality of the phenotype, muscle mass was reduced by $60 \%$ (Figure 3.5 a).

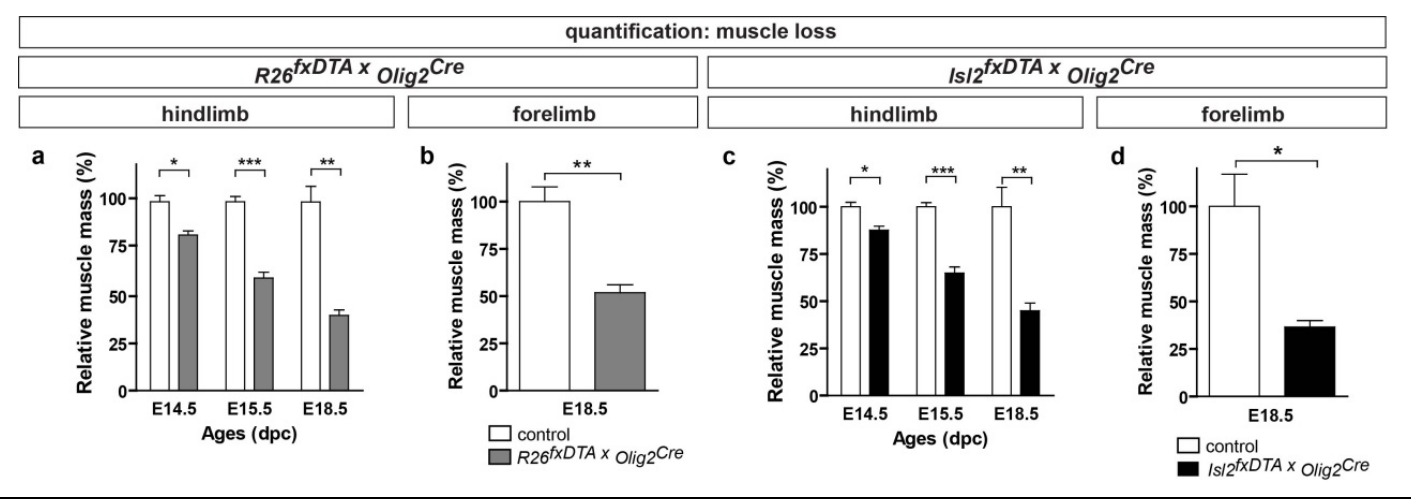

Figure 3.5: Quantification of muscle loss in $\mathrm{R}^{66^{f x D T A}} x \mathrm{Olig} 2^{\mathrm{Cre}}$ and Isl2 ${ }^{\text {fxDAA }} \mathrm{xOlig} 2^{\mathrm{Cre}}$ mice.

(a) In the hindlimb, muscle loss starts at E14.5 and progresses gradually until E18.5, the end stage of R26 ${ }^{f x D T A} x O \operatorname{Oig} 2^{\mathrm{Cre}}$ and Isl2 ${ }^{\mathrm{x} D T A} x \mathrm{Olig} 2^{\mathrm{Cre}}$ mice. (b) Quantification of cross sectional area for muscles remaining in the distal forelimb of $\mathrm{R} 26 \mathrm{FDTA} x \mathrm{Olig} 2 \mathrm{Cre}^{\mathrm{f}}$ mice at E18.5 revealed that mutant muscles were reduced by about $48 \%$. (c-d) Quantification of (c) hind- and (d) forelimb muscles in Isl2fxDTA $x$ Olig2 ${ }^{\text {Cre }}$ mice showed progressive muscle loss with a similar pattern that was observed in $R 26^{f x D T A} x \mathrm{Olig} 2^{C r e}$ mice. The severity of muscle loss is not determined by whether motor neurons were ablated at progenitor- or at postmitotic-stage. Data are presented as mean \pm SEM (two-tailed Student's $t$ test with unequal variance, $\mathrm{n}=3$ per genotype, per stage). ${ }^{* *}, \mathrm{p}<0.001 ;{ }^{* *}, \mathrm{p}<0.01 ;{ }^{*}, \mathrm{p}<0.05$. dpc, days of gestation.

Both $\mathrm{R} 26^{\text {xxDTA}} x \mathrm{Olig} 2^{\mathrm{Cre}}$ and Isl2 ${ }^{\text {fxDTA }} x \mathrm{Olig} 2^{\mathrm{Cre}}$ mice displayed a highly similar pattern of muscle loss (Figure 3.5 a and c), taking into account that motor neurons were ablated at different time points in these two mutant mice. This suggests that the severity of muscle loss is not determined by whether motor neurons were ablated at progenitor- 
or postmitotic-stage, but rather by the fact that they were eliminated before exerting their influence on their target muscles (as can be seen that the formation and delamination of somites was not prevented). Accordingly, muscle mass remaining in the hindlimb of Isl2 ${ }^{f x D T A} x$ Olig 2 Cre mice was about $65 \%$ at E15.5 and $45 \%$ at E18.5 when normalized to age-matched controls (Figure $3.5 \mathrm{c}$ ). Forelimb sections were only examined at E18.5 and muscle mass remaining was $52 \%$ and $37 \%$ for $R 26^{f x D T A} x \mathrm{Olig} 2{ }^{\mathrm{Cre}}$ mice and Isl2 $2^{f x D T A} x \mathrm{Olig}^{\mathrm{Cre}}$ mice, respectively (Figure $3.5 \mathrm{~b}$ and $\mathrm{d}$ ). Taken together, the formation and/or maintenance of muscle requires the presence of the nerve, while the observed muscle defects are specific to the loss of motor innervation, rather the timing

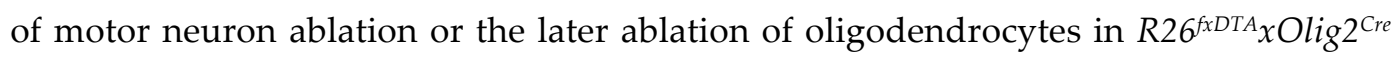
mice.

\subsubsection{Abnormal muscle fiber morphology in $\mathrm{R}^{26^{x D T A}} x \mathrm{Olig} 2^{\mathrm{Cre}}$ mice}

In the adult, myotubes in denervated and paralyzed muscles have been described to undergo atrophy induced by protein breakdown (Drachman, 1964; Giacobini et al., 1973; Hall, 1975; Pittman and Oppenheim, 1979). Contrastingly, my examination of aneural embryonic muscle fibers in $\mathrm{R}^{2} 6^{f x D T A} x \mathrm{Olig} 2 \mathrm{Cre}$ mice revealed an unexpected increase in muscle fiber cross sectional area (CSA). Muscle fibers from two muscles, tibialis anterior (TA; an extensor muscle) and soleus (S; a flexor muscle) were examined at E14.5 and E18.5. At E14.5, the diameter of mutant muscle fibers (Figure $3.6 \mathrm{~b}$ and $\mathrm{d}$ ) appeared slightly larger when compared to control muscle fibers (Figure 3.6 a and c). Mutant muscle fibers from both muscles showed a similar phenotype and seemed to be affected equally. This aberrance was further investigated at E18.5, where the increase in CSA of mutant muscle fibers became more evident as muscle fibers appeared "swollen", varied in size and were irregularly shaped (Figure 3.6 e-h). In addition to an increase in CSA, mutant muscle fibers also displayed a juvenile 
appearance with centralized cell nuclei (Figure $3.6 \mathrm{f}$ and $\mathrm{h}$ ), instead of the peripheral localization of nuclei in mature control fibers (Figure $3.6 \mathrm{e}$ and g).

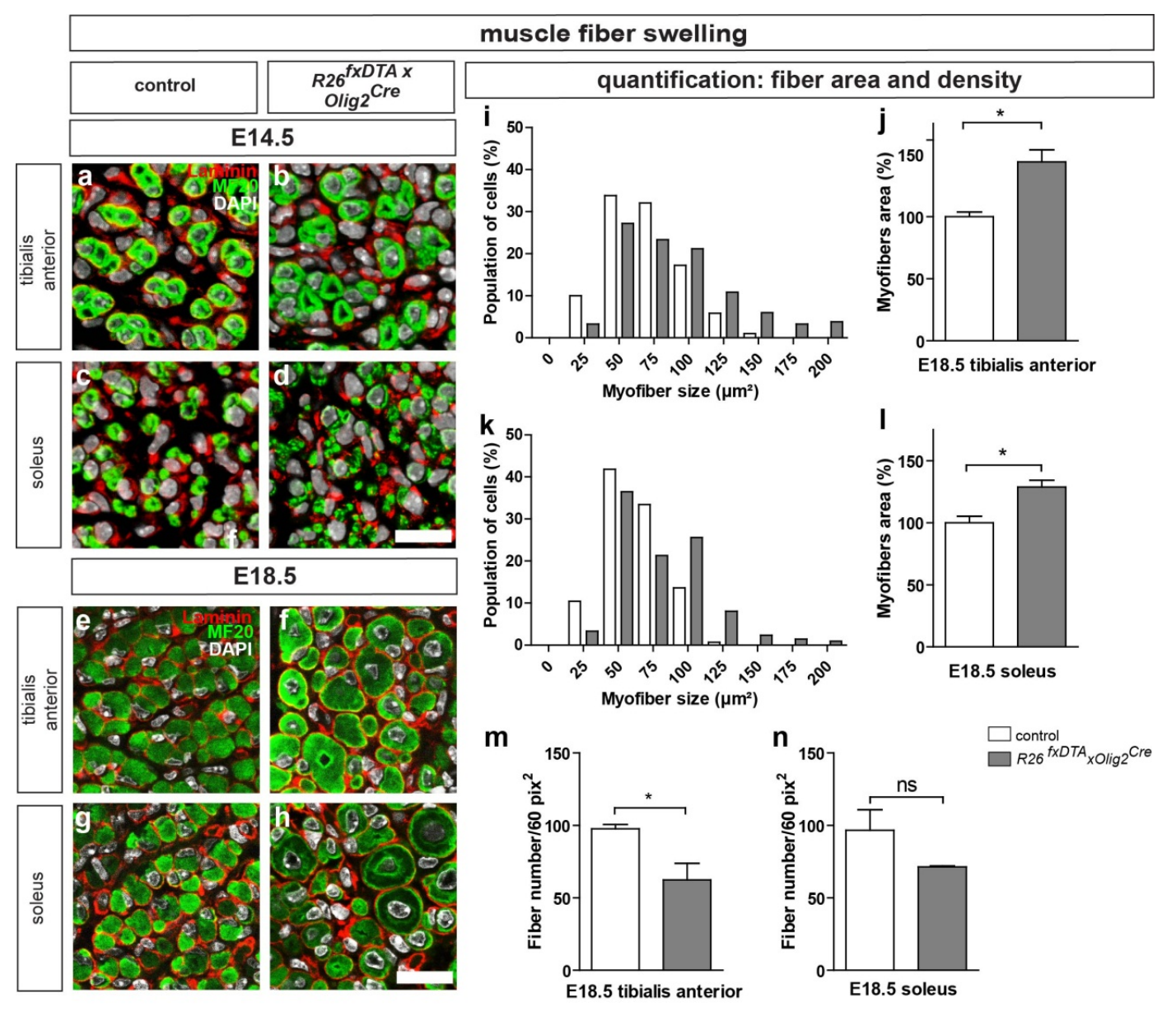

Figure 3.6: Muscle fibers undergo hypertrophy after motor neuron ablation.

(a-d) Transverse section of E14.5 TA muscle fibers from (a) control and (b) R26fxDTA xOlig2 ${ }^{\text {Cre }}$ mice stained with antibodies specific for laminin, MF20. Nuclei were stained with DAPI. (c-d) Transverse section of E14.5 control and mutant fibers within S muscle. (e-h) Transverse section of E18.5 TA muscle fibers from (e) control and (f) R2 $6^{\text {fDTA } x O l i g 2 C r e}$ mice. Cross section of E18.5 (g) control fibers and (h) mutant fibers within S muscle. Mutant fibers displayed an evident increase in fiber diameter and have central nuclear localization. (i, k) Size distribution of E18.5 (i) TA and (k) S muscle fibers. (j, 1) Quantification of muscle fiber cross sectional area (CSA) in E18.5 (j) TA and (l) S muscle. (m-n) Fiber density quantification within (m) TA and (n) S muscle at E18.5. Data are presented as mean \pm SEM (two-tailed Student's $t$ test with unequal variance, $\min \mathrm{n}=3$ per genotype) and as normalized values to control. ${ }^{* *}, \mathrm{p}<0.001 ;{ }^{* *}, \mathrm{p}<0.01$; , $\mathrm{p}<0.05$. All scale bars: $20 \mu \mathrm{m}$. 
Morphometric quantification also revealed a shift in the size distribution of atypical mutant muscle fibers from both TA and S muscle towards larger diameters (Figure 3.6 $i$ and $k$ ), supporting the finding that the average muscle fiber CSA in mutant mice was increased by $44 \%$ and $29 \%$ in the TA and S muscle, respectively (Figure $3.6 \mathrm{j}$ and 1 ). Muscle fiber density, the measure of number of muscle fibers contained within a defined area was also significantly reduced by $38 \%$ within mutant TA muscle (Figure $3.6 \mathrm{~m}$ ). However, no significant difference in fiber density was detected within $\mathrm{S}$ muscle of mutant mice with a $29 \%$ reduction (Figure $3.6 \mathrm{n}$ ). These qualitative and quantitative data thus show that both myotube size and density are profoundly affected by innervation.

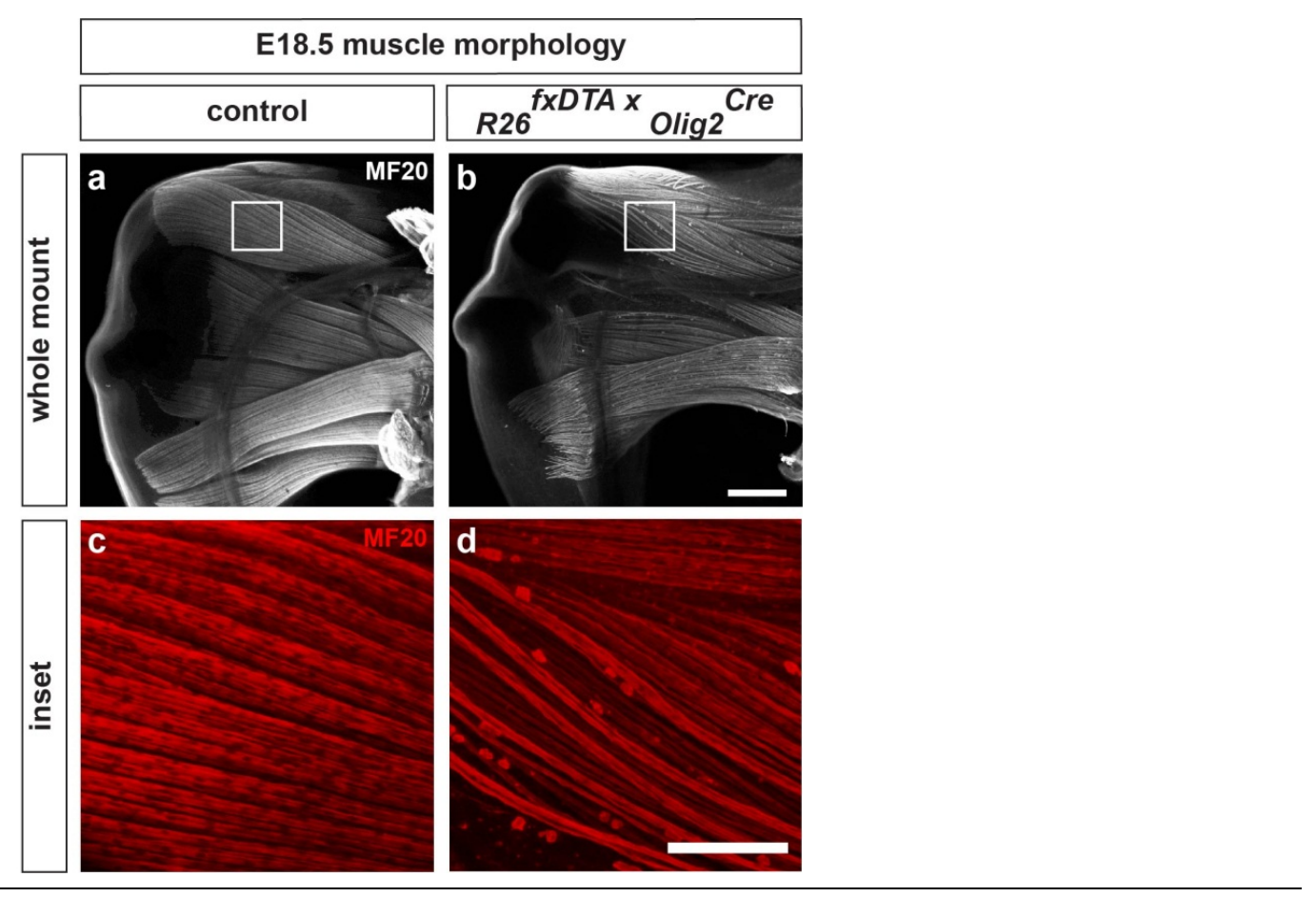

Figure 3.7: Abnormal myofiber morphology and organization in aneural mice.

(a-b) E18.5 ventral hindlimb of (a) control and (b) R26fxDTA xOlig2 ${ }^{\text {Cre }}$ mice stained with MF20. Mutant hindlimbs appeared smaller compared to controls. (c-d) Insets of boxed areas show representative higher magnification images of whole mount hindlimb preparation of (c) control and (d) $R 26^{f x D T A} x O l i g 2^{C r e}$ mice. The presence of debris from fragmented muscle fibers, suggests ongoing degeneration. All scale bars: $500 \mu \mathrm{m}$. 
To further characterize possible morphological defects caused by removal of motor neurons, I performed whole mount immunostaining to visualize skeletal muscle fibers in hindlimbs derived from control and $226^{f x D T A} x \mathrm{Olig} 2^{\text {Cre }}$ mice. Hindlimbs from mutant mice were noticeably thinner and irregular in appearance (Figure $3.7 \mathrm{~b}$ ), and contained less muscle mass compared to control hindlimbs (Figure 3.7 a). A higher magnification view revealed that aneural muscle fibers were sparse and coarser in appearance (Figure $3.7 \mathrm{~d}$ ), consistent with previous results. Furthermore, compared to controls,

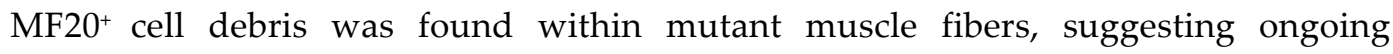
degeneration of muscle fibers (Figure $3.7 \mathrm{c}-\mathrm{d}$ ).

\subsubsection{Loss of motor neurons in mutants also leads to enhanced cell death in skeletal muscles}

It has long been established that the maintenance of skeletal muscle fibers requires innervation (McLennan, 1994). Denervation in the adult leads to structural and cellular changes in the integrity of a muscle fiber, ultimately leading to muscle atrophy mainly caused by ubiquitin-mediated proteolysis via the proteasome (Beehler et al., 2006). Degeneration has also been documented in early embryonic denervation studies (Fredette and Landmesser, 1991a; Wilson and Harris, 1993). Consistently, muscle mass reduction and degenerative signs were observed upon motor neuron ablation in R2 $6^{f x D T A} x$ Olig $2^{\text {Cre }}$ and Isl2 ${ }^{\mathrm{fxDTA}} x \mathrm{Olig} 2^{\mathrm{Cre}}$ mice (Figure 3.5 and 3.7). I hypothesized that increased cell death of muscle fibers or myogenic cells could contribute to the muscle mass reduction. To test this hypothesis, muscle sections were stained with an antibody specifically recognizing cleaved caspase 3. Caspase 3 is an executioner caspase implicated in the apoptosis and activation of caspase 3 induces a cell to follow a preprogrammed pathway of self-destruction. A significant increase in cleaved caspase $3^{+}$cells was detected within muscle fibers of $\mathrm{R} 26^{f x D T A} x \mathrm{Olig} 2^{\mathrm{Cre}}$ and $\mathrm{Isl} 2^{\mathrm{fxDTA}} x \mathrm{Olig} 2^{\mathrm{Cre}}$ mice 
(Figure $3.8 \mathrm{~b}$ and c), when compared to a basal level of apoptotic cells that was present within control muscle fibers (Figure 3.8 a).

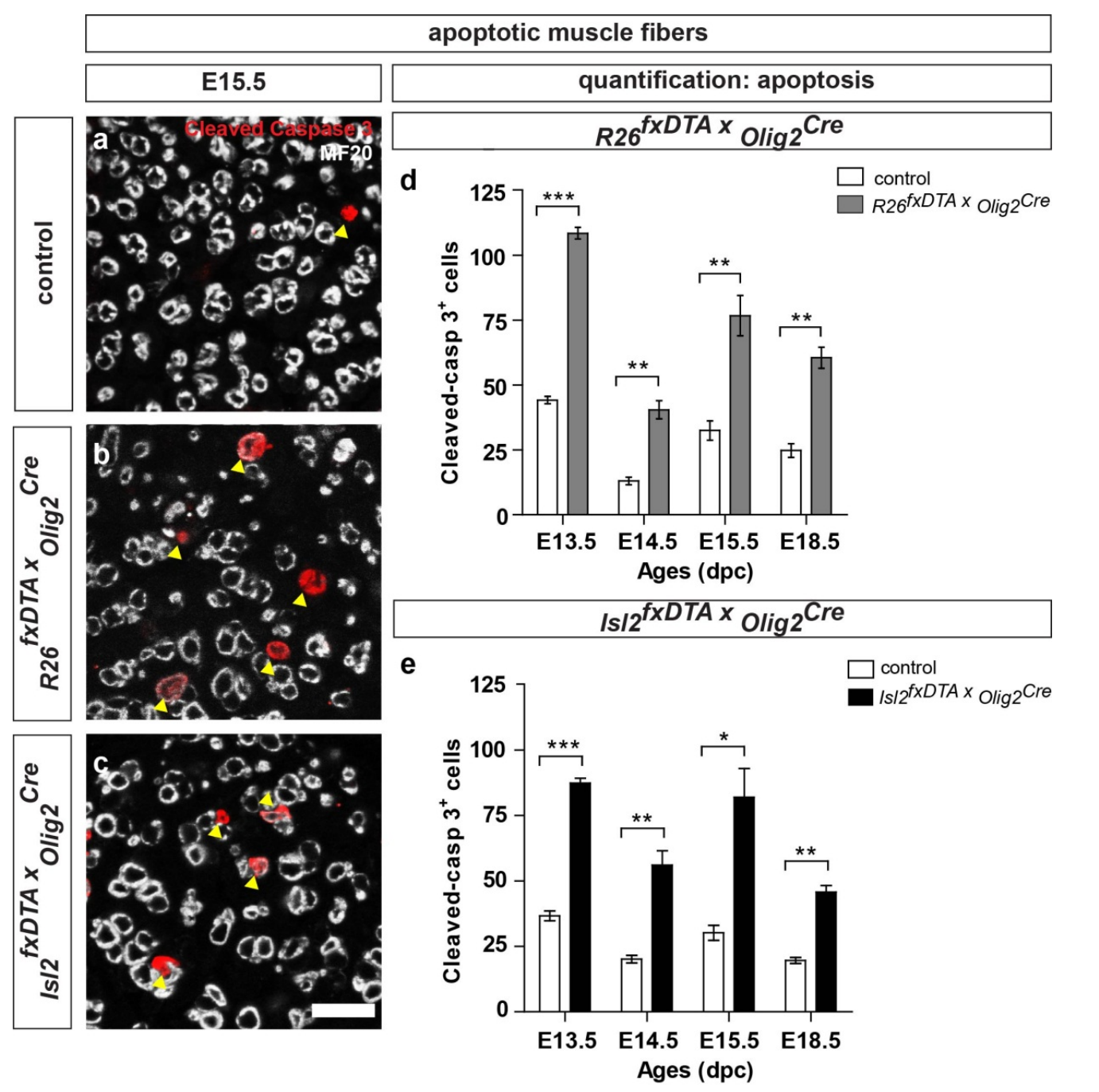

Figure 3.8: Aneural muscle fibers undergo progressive apoptosis.

(a-c) High magnification view of co-immunohistochemistry on E15.5 (a) control, (b) R26fxDTA $x$ Olig2 Cre and (c) Isl2 ${ }^{f x D T A} x O \operatorname{Oig} 2^{C r e}$ transverse distal hindlimb sections. Cleaved caspase $3^{+}$cells were elevated within muscle fibers of both mutant mice. Arrowheads point to apoptotic cells. Scale bar: $20 \mu \mathrm{m}$. (d-e) Quantification of cleaved caspase $3^{+}$cells in control, (d) $\mathrm{R}^{2} 6^{f x D T A} x \mathrm{Olig} 2^{\mathrm{Cre}}$ and (e) Isl2 fxDTA $x \mathrm{Olig} 2^{\mathrm{Cre}}$ transverse distal hindlimb muscle masses at different developmental stages. Data are presented as mean \pm SEM (two-tailed Student's $t$ test with unequal variance, $\min \mathrm{n}=3$ per genotype, per stage). ${ }^{* * *}$, $\mathrm{p}<0.001 ;{ }^{* *}, \mathrm{p}<0.01 ;{ }^{*}, \mathrm{p}<0.05$. Cleaved caspase 3 , early cell apoptosis marker. 
The number of apoptotic cells within muscle fibers throughout different developmental stages was quantified in $\mathrm{R}_{2} 6^{f \times D T A} x \mathrm{Olig} 2^{\mathrm{Cre}}$ and Isl2 ${ }^{\mathrm{fxDTA}} x \mathrm{Olig} 2^{\mathrm{Cre}}$ mice (Figure $3.8 \mathrm{~d}$ and e). E13.5 was the earliest possible time point that could be investigated after muscle cleavage completion. In control embryos, two waves of apoptosis could be observed at E13.5 and E15.5, which can be attributed to normal levels of developmental apoptosis. Throughout all developmental stages analyzed, $R 26^{\text {xxDTA }} x \mathrm{Olig} 2^{\mathrm{Cre}}$ embryos exhibited a significantly higher number of apoptotic cells compared to control embryos. Accordingly, on transverse distal hindlimb sections of $R 26^{\text {fxDTA }} \mathrm{xOlig} 2^{\text {Cre }}$ mice, apoptotic cells were increased by about $2.45-, 3.10-, 2.36-$ and 2.44-fold when compared to control mice at E13.5, E14.5, E15.5 and E18.5, respectively (Figure $3.8 \mathrm{~d}$ ). At the same time, in $\mathrm{Isl2}^{\mathrm{f} \times \mathrm{DTA}} x \mathrm{Olig}_{2}{ }^{\mathrm{Cre}}$ mutant and control mice a pattern of ongoing apoptosis virtually identical to $\mathrm{R}^{66^{f x D T A}} x \mathrm{Olig} 2^{\mathrm{Cre}}$ mice was detected (Figure 3.8 e). In the former, apoptotic cells were elevated by about 2.38-, 2.78-, 2.71- and 2.33fold when compared to control mice at E13.5, E14.5, E15.5 and E18.5, respectively (Figure 3.8 e). Consistently, terminal deoxynucleotidyl transferase dUTP nick end labeling (TUNEL) assay, a standard test for DNA fragmentation associated with apoptotic nuclei was also performed. The number of TUNEL ${ }^{+}$nuclei was also found to be elevated in muscle fibers of mutant mice (data not shown). Thus, it can be deduced that skeletal muscle hypoplasia triggered by motor neuron ablation involves apoptosis.

\subsubsection{Slow myosin pattern is relatively preserved in aneural muscles}

An important aspect of muscle development is the diversification of myofibers into different fiber types (Rubinstein and Kelly, 1978; Gunning and Hardeman, 1991; Schiaffino and Reggiani, 1996). 


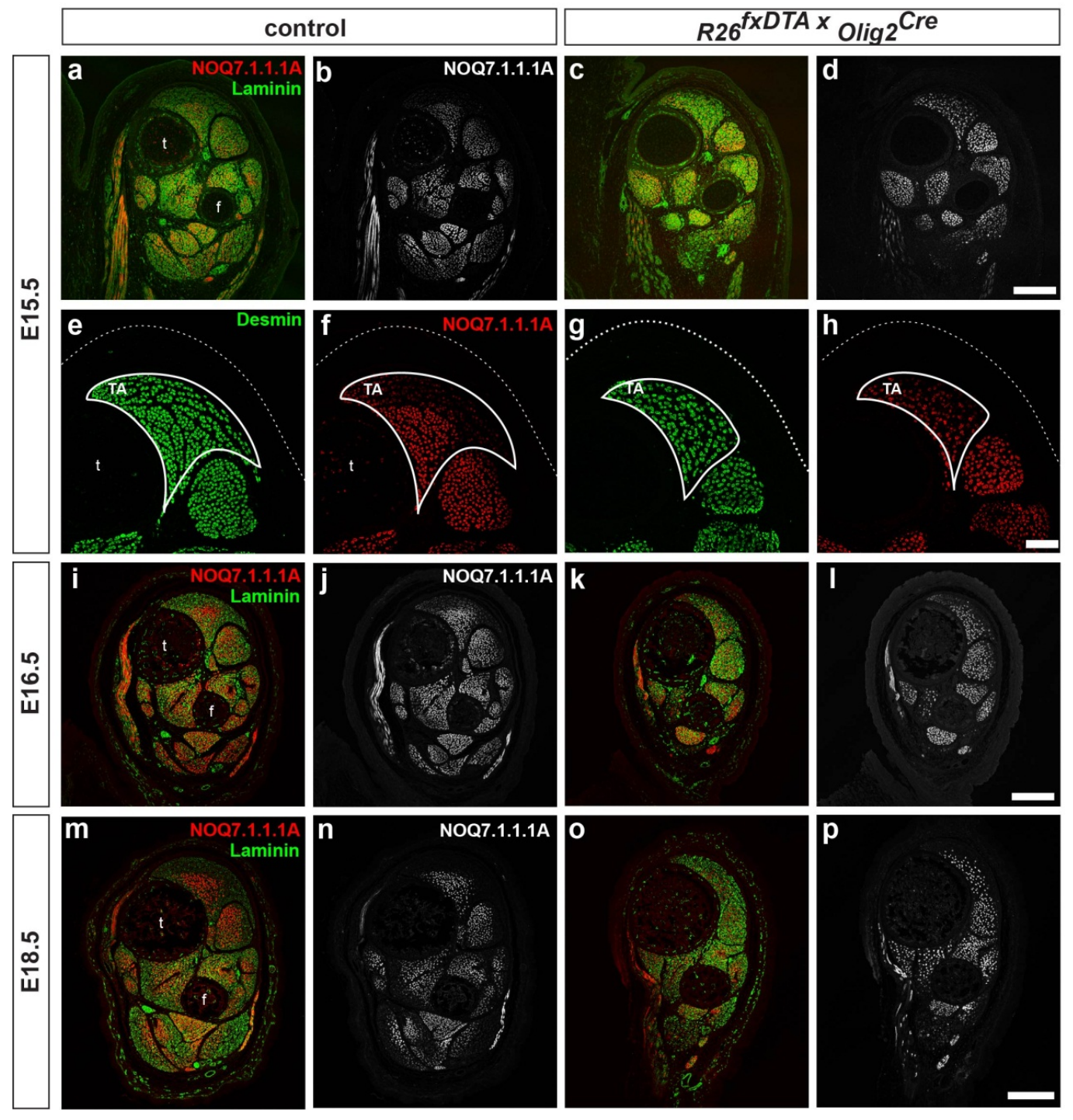

Figure 3.9: Slow myosin pattern was preserved in aneural muscles until E18.5.

(a-d) E15.5 transverse distal hindlimb section showing (a, c) general and (b, d) slow muscle pattern in (a-b) control and (c-d) $R 26^{f x D T A} x$ Olig $2^{C r e}$ mice Scale bar: $250 \mu \mathrm{m}$. (e-h) Higher magnification view of E15.5 transverse TA muscle stained with $(\mathrm{e}, \mathrm{g})$ desmin and $(\mathrm{f}, \mathrm{h})$ slow myosin antibody showing general muscle loss in (g) 2 $^{f x D T A} x$ Olig2 ${ }^{\text {Cre }}$ when compared to (e) control mice. The distribution of slow MyHC ${ }^{+}$ fibers within mutant TA muscle was altered in mutants. Bold lines demarcate TA muscle and dotted lines indicate border of hindlimb Scale bar: $100 \mu \mathrm{m}$. (i-1) Transverse distal hindlimb section showing E16.5 (i, k) general and (j, l) slow muscle pattern in (i-j) control and (k-1) R26 fxDTA $x$ Olig2 ${ }^{\text {Cre }}$ mice. Scale bar: $250 \mu \mathrm{m}$. (m-p) Transverse distal hindlimb section showing E18.5 (m, o) general and (n, p) slow muscle pattern in (m-n) control and (o-p) R2 $6^{\text {FDTA } x O l i g 2^{C r e}}$ mice. Slow myosin pattern is relatively preserved in (h) mutants, similar to (f) control mice until E18.5. Desmin, general muscle marker; NOQ7.1.1.1A, slow myosin marker. Scale bar: $250 \mu \mathrm{m}$. 
It is accepted that adult slow fibers arise from primary myotubes which express slow myosin (slow $\mathrm{MyHC}^{+}$), whereas myotubes generated mostly from the second wave of myogenesis do not express slow MyHC and will later differentiate into adult fast fibers (Jansen and Fladby, 1990; Lyons et al., 1990). To examine the impact of motor neuron ablation on primary myotube generation, transverse distal hindlimbs were coimmunostained with antibodies against slow $\mathrm{MyHC}$ and laminin. At all stages examined (E15.5-E18.5), an overview of transverse hindlimb sections revealed a reduction in general muscle mass (Figure $3.9 \mathrm{a}-\mathrm{p}$ ). Despite this, slow $\mathrm{MyHC}^{+}$fibers (primary myotubes) were found in muscles of $\mathrm{R} 26^{f x D T A} x \mathrm{Olig} 2^{\mathrm{Cre}}$ mice, confirming that primary myotubes were formed aneurally. Moreover, the distribution of slow $\mathrm{MyHC}^{+}$ fibers appeared preserved within $\mathrm{R}_{2} 6^{f x D T A} x \mathrm{Olig} 2{ }^{\mathrm{Cre}}$ muscles throughout embryogenesis (Figure $3.9 \mathrm{~d}, \mathrm{~h}, 1$ and p). This is also evidenced by quantification of the ratio of slow $\mathrm{MyHC}^{+}$laminin $^{+}$muscle fibers to all laminin ${ }^{+}$fibers at E18.5 (Figure $3.10 \mathrm{a}$ and b).

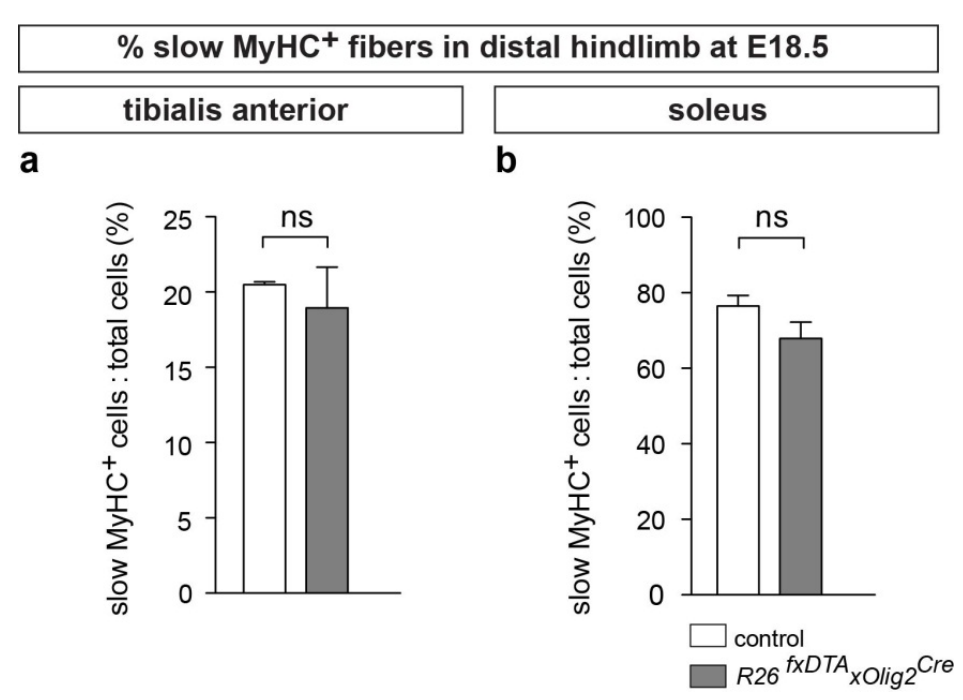

Figure 3.10: Quantification of slow $\mathrm{MyHC}^{+}$fibers in $\mathrm{R}^{66^{f x D T A}} \mathrm{xOlig2}^{\mathrm{Cre}}$ mice.

No significant differences could be detected in proportion of slow $\mathrm{MyHC}^{+}$to all laminin ${ }^{+}$muscle fibers between (a) TA and (b) S muscles of control and mutant mice. Data are presented as mean \pm SEM (twotailed Student's $t$ test with unequal variance, $n=3$ per genotype). ns, $p>0.05$. MyHC, myosin heavy chain. 
From the observation that muscle mass in $R 26^{f x D T A} x O \operatorname{Oig} 2^{\text {Cre }}$ mice was lost by $10 \%$ at E14.5 (the stage prior to initiation of secondary myogenesis), it can be inferred that existing primary myotubes undergo degeneration in the absence of innervation. Additionally, although slow $\mathrm{MyHC}^{+}$fiber distribution was relatively preserved in hindlimb muscles of aneural $R 26^{f x D T A} x O \operatorname{lig} 2^{\text {Cre }}$ embryos, the TA muscle was an exception where slow $\mathrm{MyHC}^{+}$fiber distribution was altered (refer to figure legend, Figure $3.9 \mathrm{f}$ and $\mathrm{h}$ ). Staining by desmin, a pan myofiber marker, revealed general muscle reduction in TA but did not detect a separation in the two regions of the TA muscle (Figure 3.9 e and g), indicating that the change was specific to slow $\mathrm{MyHC}^{+}$ distribution. Taken together, the relatively preserved slow MyHC pattern in hindlimbs suggests that myogenesis in the absence of innervation did not have severe impacts on primary fiber specification or formation. The alteration in TA muscle pattern could reflect (1) degeneration of myotubes (primary and secondary), and/or (2) a decreased capability of aneural $R 26^{f x D T A} x O \operatorname{Oig} 2^{\text {Cre }}$ muscle progenitors to undergo secondary myogenesis, hence resulting in fewer slow MyHC- secondary myotubes.

\subsubsection{Both primary and secondary myogenesis are affected by removal of motor neurons}

Secondary fibers, generated later, are known to make up the main bulk of muscles (Ashby et al., 1993b) and are proposed to be independent of innervation (Condon et al., 1990; Fredette and Landmesser, 1991a). To determine if secondary myotubes are generated in the absence of motor neurons and the impacts of motor neuron removal has on secondary myogenesis, assessment of the proliferation of primary and secondary muscle fibers was necessary. However, there are currently no markers to clearly distinguish between the two types of muscle fibers, although it is known that they express different MyHC isoforms (Furst et al., 1989; Lyons et al., 1990). Presently, most studies relied on an indirect "exclusion" method to separately identify fibers 
primary slow $\mathrm{MyHC}^{+}$from secondary slow MyHC-muscle fibers. To achieve this, EdU (5-ethynyl-2'-deoxyuridine) labeling which is a novel alternative for measuring newly synthesized DNA synthesis during the S-phase of cell cycle was performed. Intraperitoneal injection of EdU into pregnant mice at desired post conception stages (to cover the onset of secondary myogenesis in embryos, EdU injection was done at both 14.5 and $15.5 \mathrm{dpc}$ ) will lead to the incorporation of EdU, a nucleoside analog to thymidine into DNA of embryos during active DNA synthesis (Figure $3.11 \mathrm{a}-\mathrm{d}$ ).

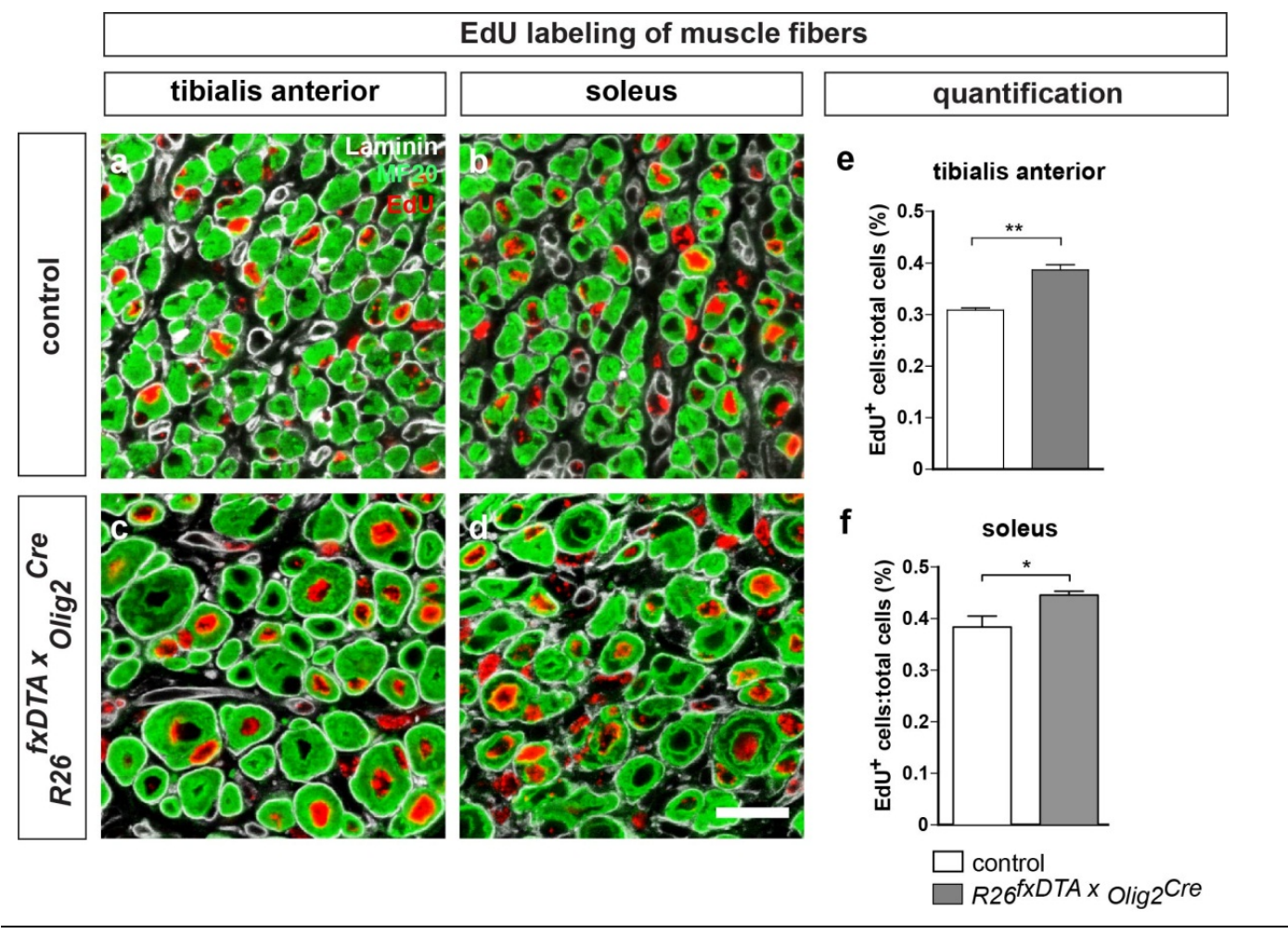

Figure 3.11: Secondary myogenesis occurs in aneural muscles.

(a-d) Co-immunohistochemistry on E18.5 transverse distal hindlimb sections of (a, c) TA and (b, d) S muscle from ( $a-b)$ control and (c-d) $R 26^{f x D T A} x$ Olig2 $2^{\text {Cre }}$ mice. Sections were obtained from embryos of pregnant mice injected with EdU at E14.5 and E15.5. Nuclei of muscle fibers generated during secondary myogenesis were incorporated with EdU. Secondary muscle fibers were detected in muscles of both control and mutant mice. Scale bar: $20 \mu \mathrm{m}$. (e-f) Quantification of EdU+ nuclei on E18.5 transverse sections of (e) TA and (f) $\mathrm{S}$ muscle. Mutant mice displayed an increase in EdU+ cells compared to controls. Data are presented as mean \pm SEM (one-tailed Student's $t$ test with unequal variance, $\mathrm{n}=3$ per genotype). ${ }^{* * *}, \mathrm{p}<0.001 ;{ }^{* *}, \mathrm{p}<0.01 ;{ }^{*}, \mathrm{p}<0.05$. EdU, 5-ethynyl-2'-deoxyuridine. 
The detection of EdU is based on a copper catalyzed click reaction between an azide and an alkyne. Secondary myotube formation in aneural muscles of $\mathrm{R}^{66^{f x D T A} x \mathrm{Olig} 2 \mathrm{Cre}}$ mice was confirmed by the presence of $\mathrm{EdU}^{+}$muscle fibers with central nuclei (presumably secondary myotubes) in E18.5 transverse TA and S muscles sections of motor neuron-deficient $\mathrm{R}_{2} 6^{f \mathrm{DTA}} x \mathrm{Olig} 2^{\mathrm{Cre}}$ mice (Figure $3.11 \mathrm{c}-\mathrm{d}$ ), contrary to earlier reports (McLennan, 1983b; Ross et al., 1987; Wilson and Harris, 1993). This finding is supported by the fact that absolute muscle mass in $R 26^{f x D T A} x O l i g 2^{C r e}$ mice increases as development proceeds, and even though muscle growth is stunted when compared to control mice; this increase in muscle mass could only be attributed to the addition of newly formed secondary fibers to the muscle bulk. This conclusion applies at least to earlier stages, before the emergence of satellite cells at E16.5 that could potentially be activated to differentiate into muscle fibers to contribute to muscle mass too. Secondary myotubes were also affected by the absence of motor innervation; relative reduction of muscle mass in $\mathrm{R} 26^{\text {fxTA }} x \mathrm{Olig} 2^{\mathrm{Cre}}$ mice even after primary myogenesis has ended when compared to controls (Figure $3.5 \mathrm{a}-\mathrm{b}$ ) strongly suggests immense degradation of secondary fibers that were formed, and likely a compromised production of secondary fibers to be the underlying cause of muscle mass reduction during later embryogenesis.

To determine the number of secondary myotubes present within muscles of control and $\mathrm{R}_{2} 6^{\text {fxDTA}} \mathrm{XOlig} 2^{\mathrm{Cre}}$ mice, the proportion of $\mathrm{EdU}^{+}$nuclei to total nuclei within hindlimb sections of control and $R 26^{f x D T A} x O \operatorname{Oig} 2^{\text {Cre }}$ mice was quantified. I observed that the number of EdU+ nuclei was significantly increased in both mutant TA and $S$ muscles (Figure 3.11 e and f). Taking into account that satellite cells were also labeled by EdU during their proliferation phase initiating at E16.5 and were therefore inevitably included in the quantification of secondary myotubes performed at E18.5, I postulated that the higher number of $\mathrm{EdU}^{+}$nuclei could either (1) indicate a compensatory increase in EdU-labeled satellite cells which are present in the muscles from E16.5 onwards, or (2) suggest an enhanced generation of secondary muscle fibers 
to accommodate the early loss of muscle mass in $R 26^{f x D T A} x O \operatorname{Oig} 2^{\text {Cre }}$ mice. The latter hypothesis seems unlikely because a rescue in muscle mass was not observed throughout development, even until birth.

\subsection{6. $R 26^{\text {fxTA }} x O \operatorname{Oig} 2^{\text {Cre }}$ mice display a compensatory increase in satellite cell numbers}

Muscle satellite cells are stem cells that are responsible for postnatal muscle growth and regeneration (Kassar-Duchossoy et al., 2005; Sambasivan and Tajbakhsh, 2007; Lepper et al., 2011; Murphy et al., 2011). They can first be detected by E16.5 in the fetal muscles (Kassar-Duchossoy et al., 2005; Lepper et al., 2011). Since an increase in apoptotic muscle cells and an elevated number of EdU ${ }^{+}$nuclei was observed in $R 26^{f x D T A} x O \operatorname{Oig} 2^{\mathrm{Cre}}$ mice, it was important to explore if there is some compensatory effect mediated by satellite cells after motor neuron removal. To test this, I performed coimmunohistochemistry on transverse distal hindlimb sections of control and mutant mice by using Pax7, a known satellite cell marker and laminin, a basal lamina antibody. In agreement with results obtained from Figure 3.6, laminin immunostaining revealed large-diameter, irregularly sized mutant muscle fibers (Figure $3.12 \mathrm{~b}$ and f). Moreover, while $\mathrm{Pax}^{+}$satellite cells were encased within the basal lamina and sarcolemma of muscle fibers in control mice (Figure $3.12 \mathrm{c}$ and e), a large proportion of satellite cells detected on mutant sections developed cytoplasmiclike processes that wrap around the perimeter of single muscle fibers (Figure $3.12 \mathrm{~d}$ and $\mathrm{f}$ ). This possibly reflects their activation and hence their differentiation into muscle fibers by using existing muscle fibers as scaffolds. To determine satellite cell numbers, the proportion of $\mathrm{Pax}^{+}$cell number to total laminin ${ }^{+}$muscle fiber number was quantified. At E18.5, satellite cells were significantly elevated in both TA and S R26 ${ }^{f x D T A} x O \operatorname{Oig} 2^{\text {Cre }}$ muscle (Figure $3.12 \mathrm{~g}$ and $\mathrm{h}$ ). Hence, the lack of innervation did not negatively affect satellite cell generation, but rather increased their numbers, 
supposedly as a compensatory response towards the loss of muscle mass induced by the absence of motor neurons.

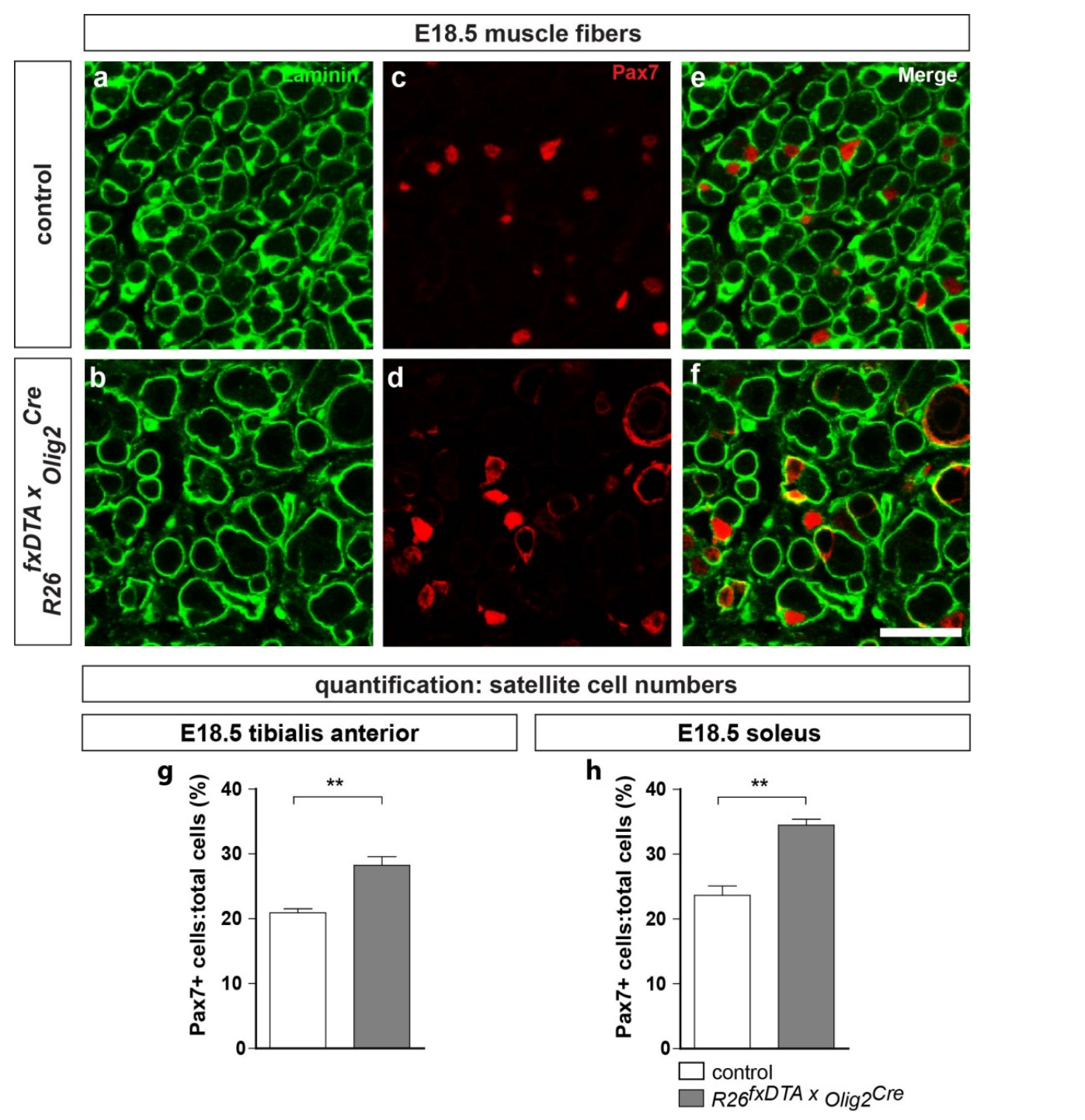

Figure 3.12: Compensatory increase in satellite cell numbers.

(a-f) Transverse distal hindlimb sections of E18.5 (a, c) control and (b, d) R26fxDTA xOlig2 ${ }^{\text {Cre }}$ mice were coimmunostained with (a-b) laminin and (c-d) Pax7, a muscle satellite cell marker. Merged coimmunostained images of (e) control (f) and $R 26^{f x D T A} x$ Olig2 $2^{C r e}$ mice. Scale bar: $20 \mu \mathrm{m}$. (g-h) Quantification of satellite cell:total laminin ${ }^{+}$cells on E18.5 transverse sections of (g) TA and (h) S muscle. Satellite cells were increased as a compensatory response to motor neuron removal. Data are presented as mean \pm SEM (two-tailed Student's $t$ test with unequal variance, $\mathrm{n}=3$ per genotype). ${ }^{* *}, \mathrm{p}<0.001 ;{ }^{* *}$, $\mathrm{p}<0.01 ;{ }^{*}, \mathrm{p}<0.05$. 
To conclude, contrary to hypotheses generated by earlier studies which proposed that only primary or secondary myotube formation are dependent on innervation (Butler et al., 1982; McLennan, 1983b; Ross et al., 1987; Fredette and Landmesser, 1991a; Hughes and Ontell, 1992; Wilson and Harris, 1993), I have shown that the formation of both primary and secondary myotubes occurs aneurally in $226^{f x D T A} x O l i g 2^{C r e}$ mice. Additionally, their continued survival depends on the presence of innervation.

\subsubsection{Motor innervation is required for myofiber terminal differentiation}

The sarcomere, being mainly composed of myosin and actin filaments, underlies the contractile properties of striated muscle fibers. I could show that primary and secondary muscle fibers formed nonetheless in embryonic muscles devoid of innervation prior to undergoing apoptosis (Figure 3.10 and Figure 3.11). Based on this, I investigated the fiber properties of existing fibers that have not yet degenerated in $R 26^{\text {fxDTA }} x \mathrm{Olig} 2^{\mathrm{Cre}}$ mice. Sarcomere markers (desmin labeling Z-band, MF20 labeling general myosin) were used to identify sarcomeres in E18.5 longitudinal muscle fibers. In control muscle fibers, sarcomeres accounting for the striated appearance to muscle fibers were prominent (Figure 3.13 a). Notably, this pattern of myosin filament intercalating between desmin ${ }^{+}$Z-bands was almost completely abolished in mutant muscle fibers (Figure $3.13 \mathrm{~b}$ ).

The absence of sarcomeres in aneurally generated myofibers could result from either (1) the failure to maintain myofiber integrity, hence leading to loss of sarcomeres, or (2) block in myofiber terminal differentiation and thus, failure to form sarcomeres. To test these possibilities, analysis of sarcomeric structures using electron transmission microscopy was performed in E15.5 embryonic limb muscles. This stage was chosen for analysis because it marks the beginning of secondary myogenesis when many myoblasts fuse together and differentiate into myotubes. 


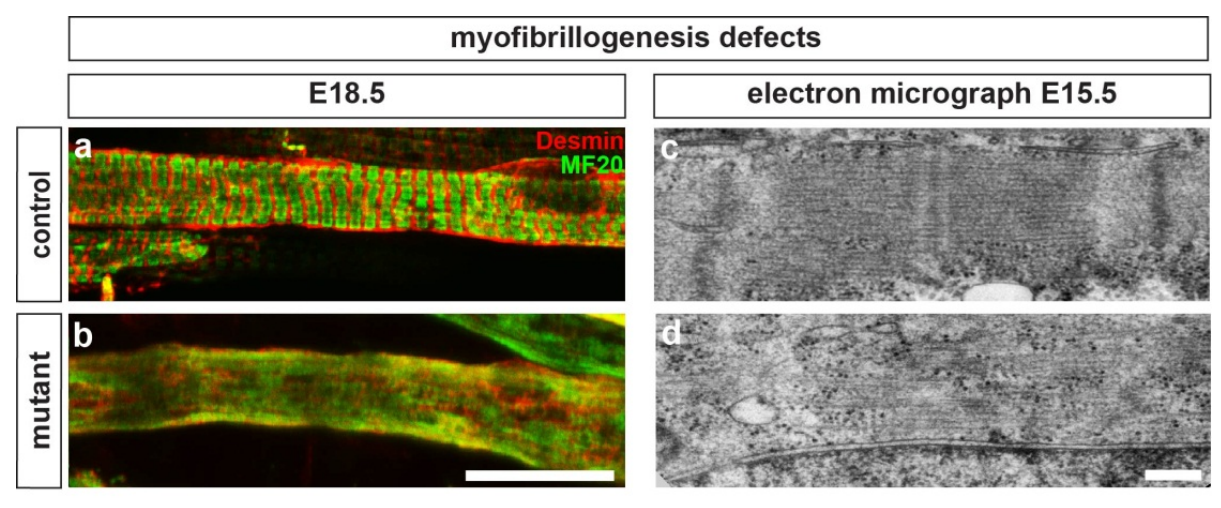

e

SARCOMERE

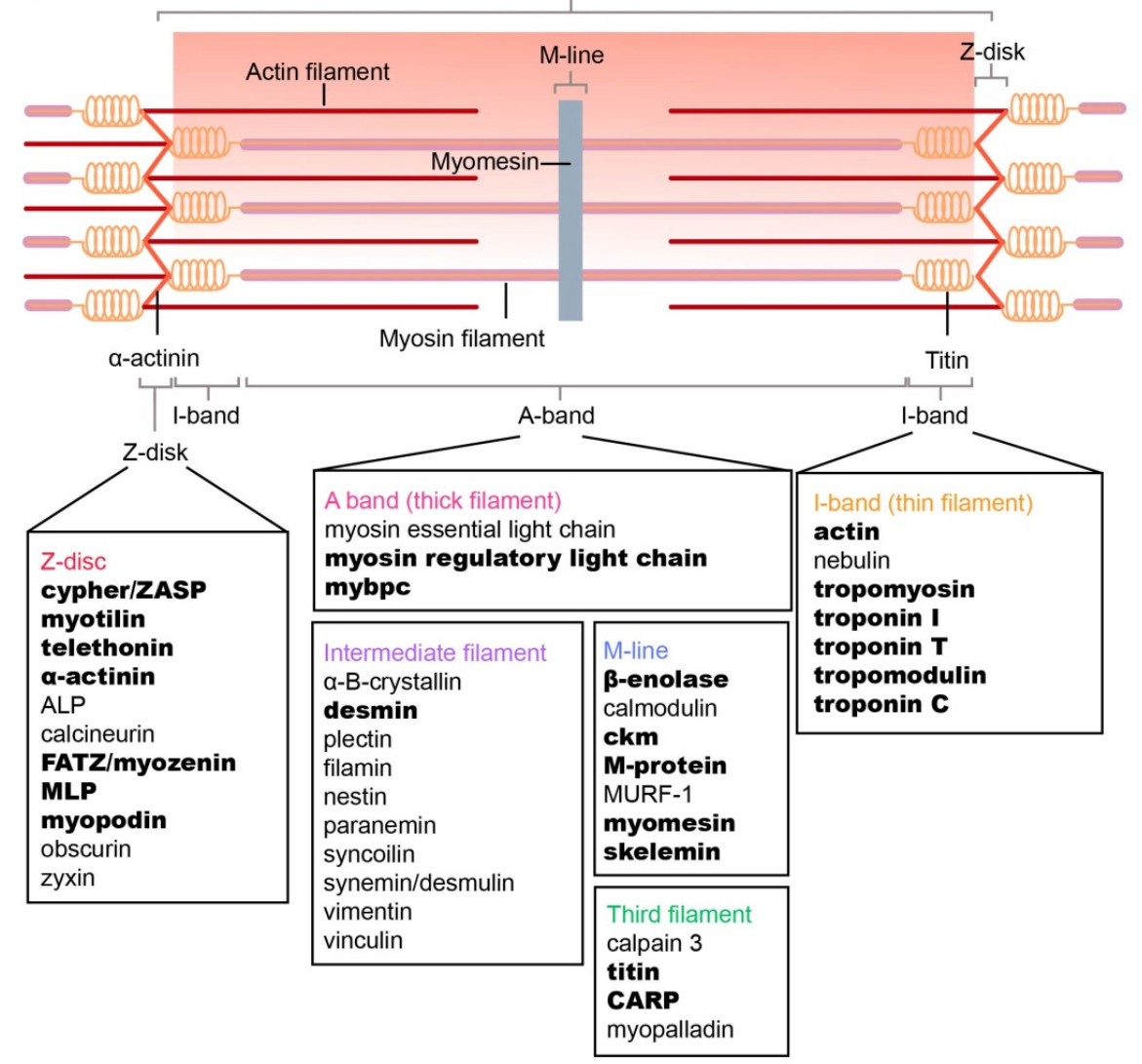

Figure 3.13: The motor nerve is required for the terminal differentiation of myotubes.

(a-b) E18.5 (a) control and (b) mutant longitudinal muscle fibers. Sarcomere pattern was almost completely abolished in R26fxDTA xOlig2Cre fibers stained with desmin and MF20. Scale bar: $20 \mu \mathrm{m}$. (c-d) Electron micrograph of E15.5 (c) control and (d) mutant muscle fibers. Besides myobrils being fewer in numbers, Z-bands and M-line were absent from the sarcomere of a single mutant fiber. Scale bar: 200 nm. (e) Known genes associated with the sarcomere. Highlighted in bold are downregulated genes in aneural muscles. Refer to Table 3.2 for expression values and statistical analysis. 


\begin{tabular}{|c|c|c|c|c|c|}
\hline Component & Gene & Control & Mutant & Fold-change & $P$-value \\
\hline \multirow{16}{*}{ Z-line } & Actn2 & 12535.53 & 7263.98 & 1.73 & $\star \star \star \star$ \\
\hline & Actn3 & 270.00 & 131.80 & 2.05 & $\star \star \star *$ \\
\hline & Cmya1 & 89.925 & 48.6 & 1.85 & $\star *$ \\
\hline & Csrp3 & 5188.00 & 1619.25 & 3.20 & 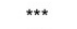 \\
\hline & $L d b 3$ & 179.98 & 68.58 & 2.62 & $\star \star \star$ \\
\hline & Myot & 164.93 & 87.93 & 1.88 & $\star \star \star$ \\
\hline & Myoz1 & 505.98 & 266.38 & 1.90 & $\star \star \star *$ \\
\hline & Myoz2 & 1136.825 & 730.5 & 1.56 & $\star \star \star \star$ \\
\hline & Obscn & -10.33 & -1.45 & 7.12 & ns \\
\hline & Pak1 & 112.625 & 100.9 & 1.12 & ns \\
\hline & Pdlim3 & 4159.85 & 2193.875 & 1.90 & $\star \star \star \star$ \\
\hline & Рpp3са & 1051.45 & 1162.15 & 1.11 & ns \\
\hline & Synpo2 & 13.80 & 2.50 & 5.52 & ns \\
\hline & Synpo21 & 197.50 & 128.43 & 1.54 & $* \star$ \\
\hline & Tcap & 750.88 & 280.78 & 2.67 & $\star \star \star \star$ \\
\hline & $Z y x$ & 16265.88 & 11138.03 & 1.46 & $\star * * *$ \\
\hline \multirow{9}{*}{ A-band } & Myh2 & 9.50 & 16.83 & 177 & ns \\
\hline & Myh4 & -1.15 & -4.10 & 3.57 & ns \\
\hline & Myh6 & 39.15 & 16.23 & 2.41 & $* \star \star$ \\
\hline & Myh7 & 83.25 & 22.80 & 3.65 & $\star \star$ \\
\hline & Myh8 & 25102.25 & 21908.93 & 1.15 & ns \\
\hline & Myl1 & 27418.15 & 27120.73 & 1.01 & ns \\
\hline & Myl2 & 4108.63 & 543.23 & 7.56 & $\star \star \star ~$ \\
\hline & Myl3 & 5947.10 & 816.45 & 7.28 & *** \\
\hline & Myl4 & 38571.10 & 33204.58 & 1.16 & $\star * \star *$ \\
\hline \multirow{6}{*}{ Third filament } & Capn3 & 35.30 & 33.30 & 1.06 & ns \\
\hline & Hspb1 & 2882.125 & 3611 & 1.25 & $\star *$ \\
\hline & Mybphl & -2.875 & -1.625 & 1.77 & ns \\
\hline & Mypn & 35.08 & 16.18 & 2.17 & $\star \star \star$ \\
\hline & Rnf34 & 1100.80 & 984.78 & 1.12 & * \\
\hline & $T t n$ & 8387.93 & 5588.40 & 1.50 & ** \\
\hline \multirow{11}{*}{ M-line } & Calm2 & 2504.20 & 2086.38 & 1.20 & * \\
\hline & Calm3 & 10434.38 & 9927.15 & 1.05 & ns \\
\hline & Calm4 & 6252.98 & 11588.33 & 1.85 & $\star \star \star ~$ \\
\hline & Calm3 & 163.80 & 287.90 & 1.76 & $\star \star$ \\
\hline & Ckm & 3648.68 & 1301.18 & 2.80 & $\star \star \star \star ~$ \\
\hline & Ckmt2 & 435.23 & 142.88 & 3.05 & 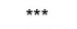 \\
\hline & Eno3 & 7418.95 & 4274.48 & 1.74 & 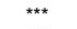 \\
\hline & Myom1 & 1195.18 & 658.80 & 1.81 & *** \\
\hline & Myom2 & -9.18 & -10.90 & 1.19 & ns \\
\hline & Smpx & 274.825 & 181.25 & 1.52 & $\star \star \star \star ~$ \\
\hline & Trim63 & 82.45 & 54.80 & 1.50 & * \\
\hline \multirow{15}{*}{ I-band } & Acta1 & 29391.58 & 14502.53 & 2.03 & $\star \star \star \star$ \\
\hline & Acta2 & 13202.95 & 11031.78 & 1.20 & $\star \star \star ~$ \\
\hline & Actc1 & 15415.23 & 14878.88 & 1.04 & ns \\
\hline & $\mathrm{Nebl}$ & -10.43 & 1.53 & 6.84 & $\star \star \star$ \\
\hline & Tmod1 & 1052.73 & 545.78 & 1.93 & *** \\
\hline & Tmod2 & 119.18 & 51.83 & 2.30 & 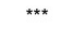 \\
\hline & Tmod3 & 764.70 & 791.20 & 1.03 & ns \\
\hline & Tmod4 & 785.30 & 382.58 & 2.05 & $\star \star \star *$ \\
\hline & Tnnc1 & 37651.50 & 31503.15 & 1.20 & * \\
\hline & Tnnc2 & 35692.53 & 26106.03 & 1.37 & $\star \star \star \star ~$ \\
\hline & Tnni1 & 746.85 & 627.23 & 1.19 & * \\
\hline & Tnni2 & 15119.30 & 10352.03 & 1.46 & ** \\
\hline & Tnnt1 & 23389.48 & 17659.23 & 1.32 & $\star \star \star$ \\
\hline & Tnnt3 & 2744.83 & 1732.18 & 1.58 & $\star \star \star \star ~$ \\
\hline & Tpm2 & 5806.80 & 3979.25 & 1.46 & $\star \star \star$ \\
\hline \multirow{9}{*}{ Intermediate filament } & Cryab & 1909.23 & 1649.93 & 1.16 & $\mathrm{~ns}$ \\
\hline & Des & 5014.43 & 3309.90 & 1.51 & 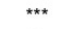 \\
\hline & Dmn & 98.65 & 82.50 & 1.20 & * \\
\hline & Mpp4 & 2.18 & 6.90 & 3.17 & ns \\
\hline & Nes & 1053.93 & 883.18 & 1.19 & * \\
\hline & Plec1 & 453.73 & 397.00 & 1.14 & ns \\
\hline & Sync & 200.40 & 144.25 & 1.39 & $\star \star$ \\
\hline & $\mathrm{Vcl}$ & 1725.45 & 1555.65 & 1.11 & * \\
\hline & Vim & 6757.10 & 7084.35 & 1.05 & ns \\
\hline
\end{tabular}

Table 3.1: List of sarcomere-associated genes in control and $\mathrm{R}^{2} 6^{\text {fxDTA } x O l i g 2^{\mathrm{Cre}}}$ mice.

Relative units of gene expression are shown under 'control' and 'mutant' field. Data under 'control' and 'mutant' field are presented as mean relative units of gene expression. (One-tailed Student's $t$ test with unequal variance, $\mathrm{n}=4$ per genotype). ${ }^{* * *}, \mathrm{p}<0.001 ;{ }^{* *}, \mathrm{p}<0.01 ;{ }^{*}, \mathrm{p}<0.05$. Red, upregulated genes $\geq 1.0$ fold. 
Electron transmission microscopy revealed that in contrast to control muscles myofibrils were fewer in numbers, while Z-bands and M-line were absent in R26 $6^{\text {fxDA }} \mathrm{xOlig} 2^{\mathrm{Cre}}$ mutants when compared to controls (Figure $3.13 \mathrm{c}-\mathrm{d}$ ). These results strongly suggest that motor innervation is critical for promoting the terminal differentiation of muscle fibers, including the formation of sarcomeres, an essential step for enabling muscle fibers to become functionally contractile.

Consistent with these results, microarray and RNAseq analysis (decribed in Section 3.4) showed that the regulation of sarcomere-associated gene expression was severely affected by the lack of motor innervation (Figure 3.13 e shows a list of sarcomereassociated genes allocated to different components of the sarcomere, whereby genes shown in bold are differentially downregulated in muscles of E15.5 R26fxDTA $x$ Olig2 ${ }^{\text {Cre }}$ mice). A large number of genes encoding proteins that are localized to distinct components of the sarcomere, particularly the Z-band, A-band, M-line and I-band were downregulated, indicating that the initiation of expression of these components is strictly dependent on motor innervation (see Table 3.1).

\subsection{Strategy to block neurotransmission genetically}

\subsubsection{Using tetanus toxin light chain to eliminate neurotransmission in motor neurons}

My results thus far show that mouse embryos in which motor neurons have been genetically removed show strongly reduced primary and secondary myogenesis and progressive loss of muscle mass due to myofiber loss. However, there are different aspects to which motor innervation can exert its influence on muscles; effects observed with these mice did not preclude influences from neural activity, nerve-derived neurotrophins, and nerve-contact. To specifically study the impacts of neural activity on muscle development, mice with Olig2 driving Cre expression were bred to $R 26^{\text {fxTeNT }}$ 
mice to generate $R 26^{f x T e N T} x O l i g 2^{\text {Cre }}$ mice with conditional neurotransmission block in motor neurons (Figure 3.14 a).

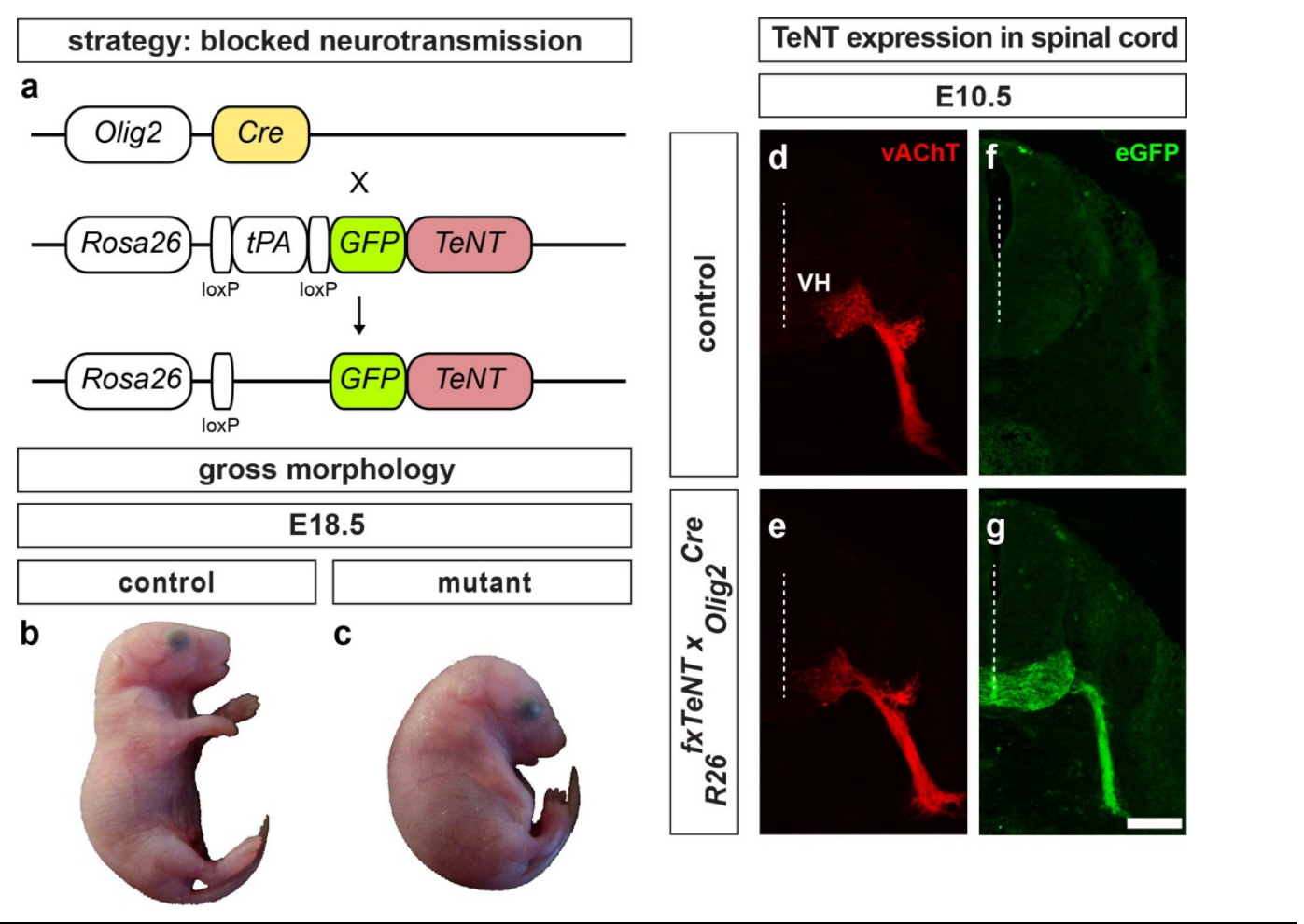

Figure 3.14: Strategy to conditionally block neurotransmission in murine motor neurons.

(a) Scheme to generate mice with blocked motor neurotransmission. Action potential-dependent vesicle release was prevented by crossing the Olig2Cre mouse line with the R26fxTeNT mouse line to generate R26 fxTeNT $x$ Olig2Cre mice. (b-c) Gross morphology of E18.5 (b) control and (c) mutant mice. Mutants displayed flaccid paralysis, remained in their fetal position and die perinatally. (d-g) No overt difference in $\mathrm{vAChT}^{+}$motor neurons at E10.5 was found between (d) control and (e) mutant mice. Axonal projections were normal for both controls and mutants. Fused GFP-TeNT protein expression was absent in the spinal cord of (e) control mice but was present in motor neuron progenitors and postmitotic motor neurons of (f) mutant mice. TeNT, tetanus toxin light chain; eGFP, enhanced GFP. Dotted lines indicate the midline of the spinal cord. Scale bar: $100 \mu \mathrm{m}$.

Tetanus light chain (TeNT) is a component of the tetanus toxin (TeTx) molecule which is derived from bacterium Clostridium tetani. TeNT cleaves synaptobrevin, a synaptic protein present on neurotransmitter-containing vesicles, thus hindering the 
association of vesicular SNARES with membrane SNARES and preventing membrane fusion as a consequence. Hence, Cre-mediated recombination will drive TeNT expression in motor neurons, thereby preventing exocytosis. To date, all studies attempting to block neurotransmission to create paralyzed muscles utilized pharmacological applications of paralyzing agents (Drachman, 1964; McLennan, 1983b; Ross et al., 1987; Fredette and Landmesser, 1991a). Similar to R26fxDTA $x$ Olig2 ${ }^{\text {Cre }}$ and Isl2 $2^{f x D A} x O \operatorname{Oig} 2^{C r e}$ mice, mutant mice die perinatally, because of respiratory failure caused by the lack of innervation to the diaphragm. Additionally, $R 26^{\text {fTeNT}} x \mathrm{Olig} 2^{\mathrm{Cre}}$ mutant mice show overall normal gross morphology, but exhibit flaccid paralysis accompanied by kyphosis-like posture compared to controls (Figure $3.14 \mathrm{~b}-\mathrm{c}$ ). To validate the specificity of TeNT expression, transverse spinal cord sections of E10.5 mice were analyzed. $R 26^{f x T e N T} x$ Olig $2^{\text {Cre }}$ mice, but not control mice, showed expression of fused green fluorescent protein (GFP) and TeNT protein in the postmitotic motor neurons as well as motor neuron progenitors. $R 26^{f x T e N T} x O \operatorname{lig} 2^{C r e}$ mice also showed no overt morphological differences to control mice (Figure $3.14 \mathrm{f}-\mathrm{g}$ ). $\mathrm{vAChT}^{+}$motor neurons were present in the ventral horn of the spinal cord and they extend their motor axons into the periphery normally (Figure $3.14 \mathrm{~d}$-e).

\subsubsection{TeNT protein is temporally expressed in mutant motor neurons throughout development}

The robust expression of fused GFP-TeNT protein detected in E10.5 mutant spinal cord prompted me to confirm the stable expression of TeNT protein in motor neurons throughout the stages that would be investigated for further muscle analysis. Transverse lumbar spinal cord sections of E12.5 embryos did not reveal any difference in $\mathrm{vAChT}^{+}$(Figure $3.15 \mathrm{a}-\mathrm{b}$ ) and Isl ${ }^{1 / 2} 2^{+}$motor neuron numbers (Figure $3.15 \mathrm{c}-\mathrm{d}$ ) between control and mutant mice. Like at E10.5, E12.5 R2 $6^{f x T e N T} x$ Olig2 ${ }^{\mathrm{Cre}}$ spinal cords were still 
distinguishable from control spinal cord by the presence of fused GFP-TeNT protein (Figure 3.15 e-f).

\begin{tabular}{|c|c|c|c|}
\hline \multicolumn{4}{|c|}{ persisting TeNT expression in motor neurons } \\
\hline control & R26 $^{\text {fxTeNT } x}$ Olig2 ${ }^{\text {Cre }}$ & control & $R_{26}{ }^{f x T e N T \times}$ Olig2 $^{C r e}$ \\
\hline
\end{tabular}
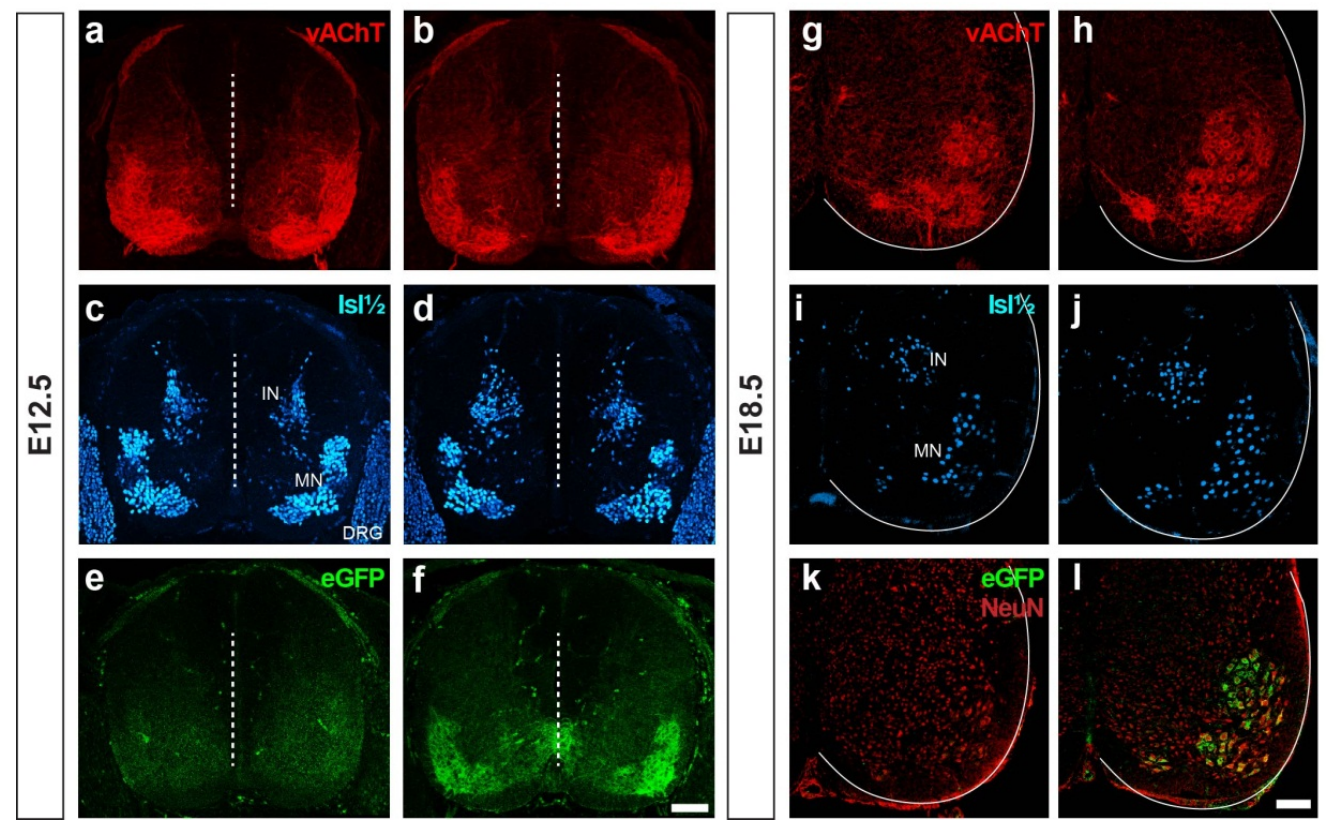

Figure 3.15: TeNT expression is specific to motor neurons.

(a-d) No overt difference could be detected on E12.5 transverse lumbar spinal cord of (a, c) control and (b, d) R26 fxTeNT $x$ Olig2 $2^{\text {Cre }}$ stained with (a-b) vAChT and (c-d) Isl1/2. (e-f) Fused GFP-TeNT protein was not detected within E12.5 spinal cord of (e) control mice, but was present in (f) mutant spinal cord. (g-j) Immunostaining of ( $\mathrm{g}-\mathrm{h}$ ) vAChT and (i-j) Isl $1 \frac{1}{2}$ on E18.5 transverse lumbar spinal cord sections revealed an increase of motor neuron numbers in $(h, j)$ mutant mice as compared to $(g, i)$ controls. (k-1) TeNT expression is consistently absent from the spinal cord of E18.5 (k) controls, but was strictly limited to motor neurons of (l) mutant mice. Dotted lines indicate midline of spinal cord, bold lines demarcate border of spinal cord. MN, motor neurons; IN, interneurons. All scale bars: $100 \mu \mathrm{m}$.

At E18.5, vAChT ${ }^{+}$lumbar motor neurons of both control (Figure $3.15 \mathrm{~g}$ ) and R26 fxTeNT $x$ Olig2 ${ }^{\text {Cre }}$ mice (Figure $3.15 \mathrm{~h}$ ) appeared to be segregated normally into respective motor columns. Strikingly, the numbers of Is $1 \frac{1}{2} 2^{+}$nuclei within the ventral part of the spinal cord were elevated in the mutants (Figure $3.15 \mathrm{j}$ ) when compared to 
controls (Figure $3.15 \mathrm{i}$ and see further below). Most importantly, fused GFP-TeNT protein was expressed stably in and was limited to E18.5 mutant motor neurons (Figure 3.15 1). Conversely, fused GFP-TeNT protein expression was undetected in age-matched control spinal cord (Figure $3.15 \mathrm{k}$ ). For all stages analyzed, TeNT expression was detected in $\mathrm{R}_{2} 6^{\mathrm{fT} T \mathrm{NT}} x \mathrm{Olig} 2^{\mathrm{Cre}}$ embryos but not in controls.

\subsubsection{Perturbing vesicular release in motor neurons results in skeletal muscle hypoplasia}

After verifying that TeNT is expressed at all embryonic developmental stages, morphometric analysis of muscles was performed on transverse distal hindlimb and forelimb sections of control and mutant mice (Figure 3.16 a-d). At E18.5, muscle groups were found to be drastically reduced or absent in the hindlimbs of

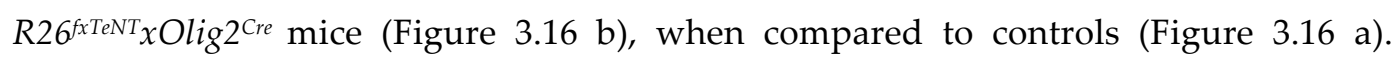
Interestingly, despite the reduction in muscle mass, cleavage of muscle groups such as the peroneal muscle was normal in $R 26^{f x T e N T} x O \operatorname{Oig} 2^{\text {Cre }}$ mice with blocked neurotransmission, whereas peroneal muscle cleavage was defective in $R 26^{f x D T A} x$ Olig2 ${ }^{C r e}$ mice lacking motor neurons. For yet unknown reasons, forelimbs were consistently less affected compared to hindlimbs in $\mathrm{R}_{26^{f x T e N T}} \mathrm{OOlig} 2^{\mathrm{Cre}}$ mice (Figure $3.16 \mathrm{c}-\mathrm{d}$ ). Muscle mass remaining in distal hindlimb at various developmental stages was quantified to determine the onset of muscle loss in $R 26^{f x T N T} x \mathrm{Olig} 2^{\mathrm{Cre}}$ mice. No significant difference between muscle masses of control and mutant mice could be detected at E14.5 and E15.5 (Figure 3.16 e), a stark contrast to mice with wholesale motor neuron removal (see previous sections). Reduction of muscle mass thus seemed to occur between E15.5 and E18.5, suggesting a link to the coinciding normal time window of alteration in pattern of spontaneous activity (Moody and Bosma, 2005). 


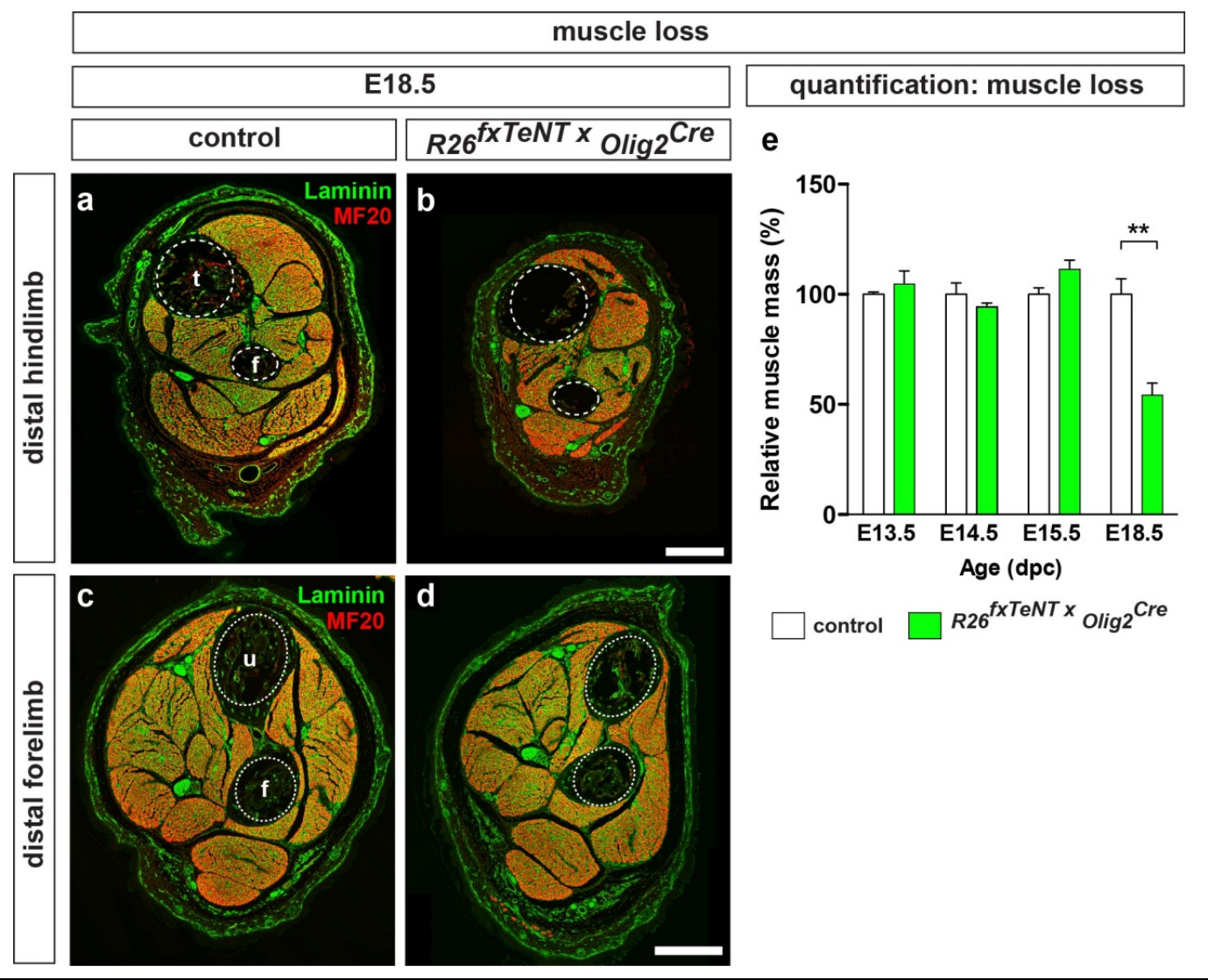

Figure 3.16: Blocked neurotransmission leads to reduced muscle mass.

(a-d) Transverse E18.5 distal (a-b) hindlimb and (c-d) forelimb of (a, c) control and (b, d) R26 fxTeNT $x$ Olig2 ${ }^{\text {Cre }}$ embryos. In the hindlimb, muscle mass in mutants was reduced in some muscle groups, whereas other muscles such as Gm and Gl were completely abolished. All scale bars: $250 \mu \mathrm{m}$. (e) Quantification of cross sectional area of hindlimb muscle mass in control and mutant mice at different developmental stages. No significant reduction in muscle mass was detected at early stages (E13.5-E15.5) whereas at E18.5, muscle mass remaining in mutant mice was $63.22 \pm 6.33 \%$ of control muscle mass. Data are presented as mean \pm SEM (two-tailed Student's $t$ test with unequal variance, $\min$ $\mathrm{n}=3$ per genotype, per stage). ${ }^{* *}, \mathrm{p}<0.001 ;{ }^{* *}, \mathrm{p}<0.01 ;{ }^{*}, \mathrm{p}<0.05$.

Apoptosis was assumed to be responsible for the loss of muscle mass in mice with perturbed neurotransmission as more apoptotic cells were detected within muscle fibers of mutant mice than controls (Figure $3.17 \mathrm{a}-\mathrm{b}$ ) at E15.5. To confirm this, the number of apoptotic cells within muscle fibers throughout different developmental stages was quantified (Figure 3.17 c). Later in development, analysis on E18.5 
transverse sections showed that cleaved caspase $3^{+}$cells were appreciably elevated in mutants by 1.65 -fold. Thus, blocking neurotransmission or completely eliminating motor neurons both appear to lead to apoptosis-mediated muscle cell death.

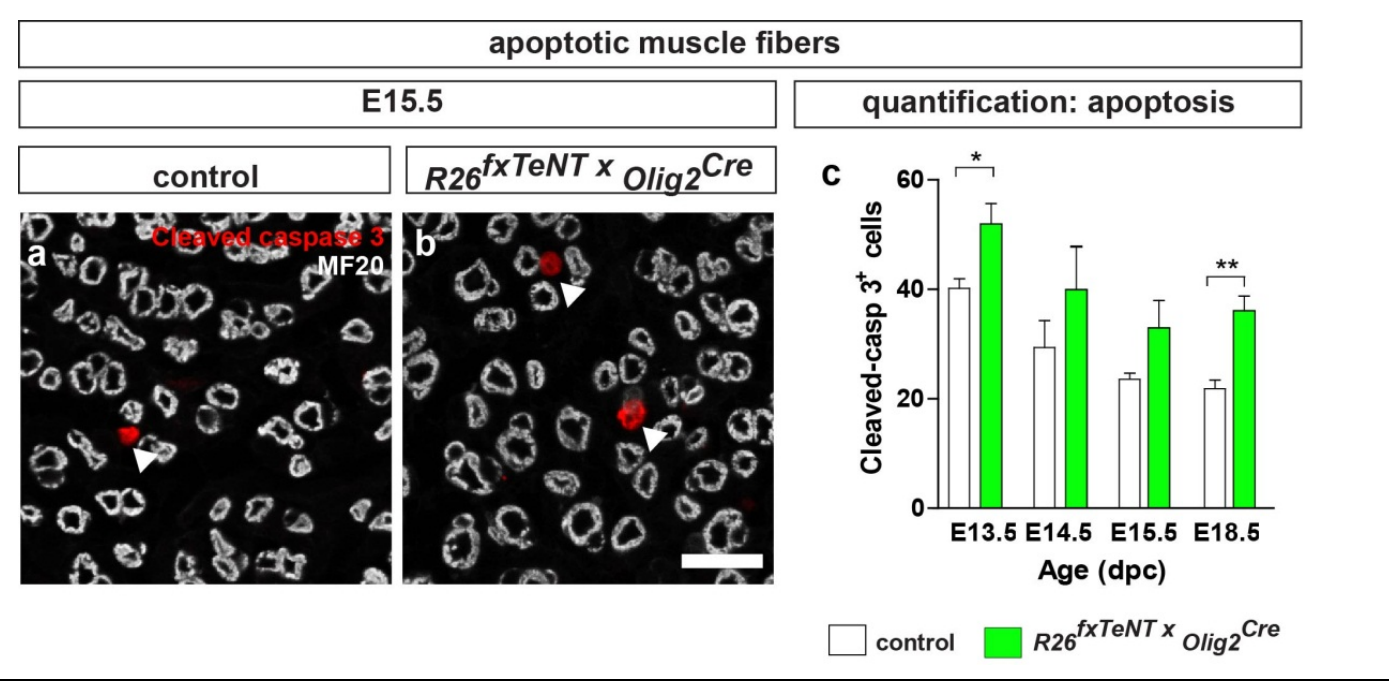

Figure 3.17: Apoptosis mediates reduced muscle mass in $\mathrm{R}^{26^{f x T e N T}} x \mathrm{Olig}{ }^{\mathrm{Cre}}$ mice.

(a-b) High magnification view of E15.5 (a) control and (b) R26 fxTeNT $x$ Olig2 ${ }^{\text {Cre }}$ transverse distal hindlimb sections. Apoptotic cells (labeled with cleaved caspase 3 and marked by arrowheads) were elevated within muscle fibers of mutant mice. Scale bar: $20 \mu \mathrm{m}$. (c) Quantification of apoptotic cells within muscle fibers of control and mutant mice at different developmental stages. At least three sections from each of three control and mutant embryos were counted for each time point. Data are presented as mean \pm SEM (two-tailed Student's $t$ test with unequal variance). ${ }^{* *}, \mathrm{p}<0.001 ;{ }^{* *}, \mathrm{p}<0.01 ;{ }^{*}, \mathrm{p}<0.05$.

\subsubsection{Slow myosin expressing myofibers are generated in paralyzed muscles}

To examine the impact of neurotransmission blockade on primary myotube generation, transverse sections of distal hindlimbs were co-immunostained with antibodies against slow myosin $(\mathrm{MyHC})$ and laminin. Like $R 26^{f x D T A} x \mathrm{Olig} 2^{\mathrm{Cre}}$ mice, myofibers expressing slow MyHC isoforms were generated within paralyzed muscles of $\mathrm{R}^{6^{f x T e N T}} \mathrm{xOlig} 2^{\mathrm{Cre}}$ mice throughout embryogenesis until E18.5. 


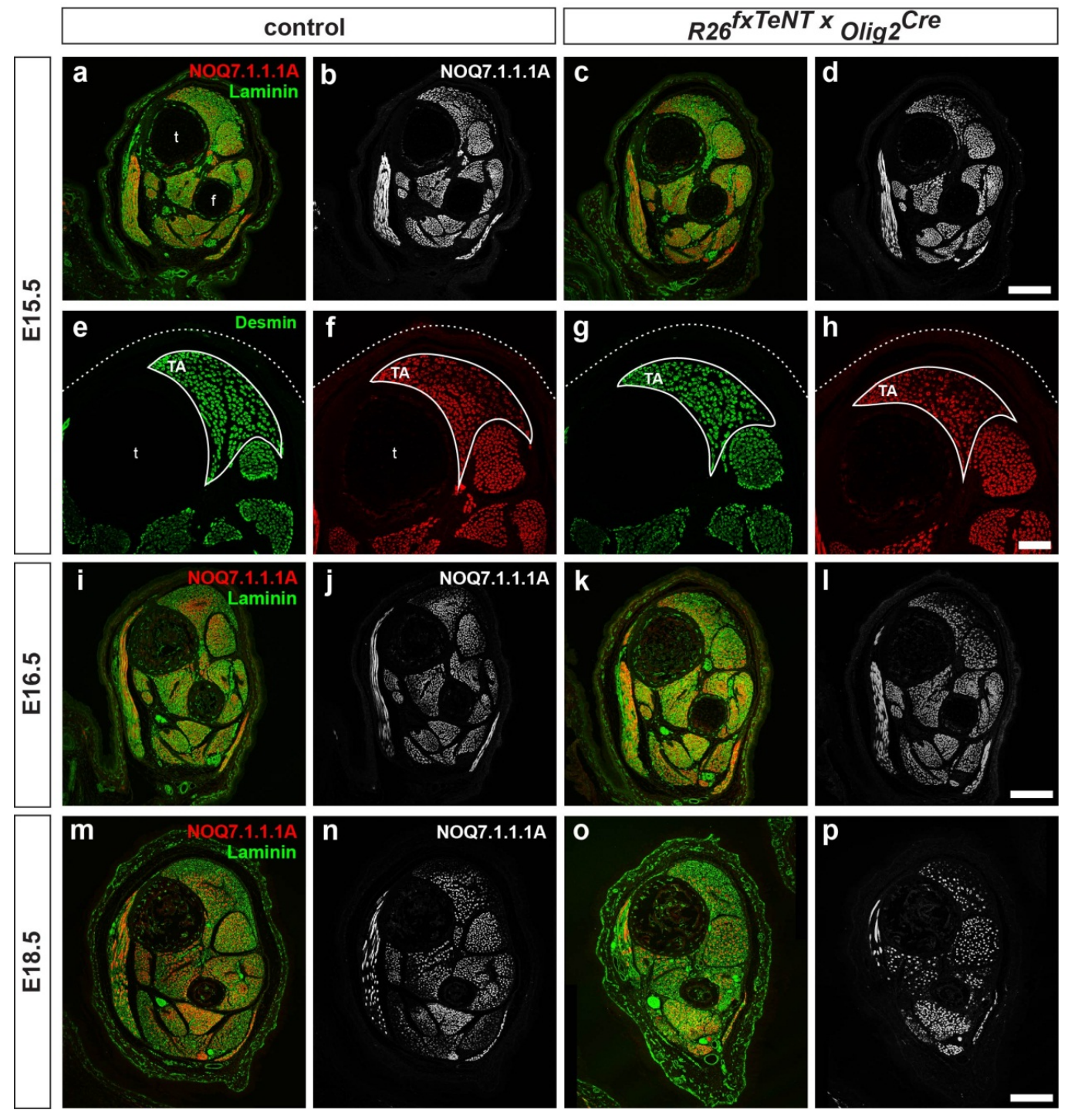

Figure 3.18: Slow myosin pattern was preserved in paralyzed muscles until E18.5.

(a-d) E15.5 transverse distal hindlimb section showing (a, c) general and (b, d) slow muscle pattern in (a-b) control and (c-d) R26 frTeNT $x$ Olig2 ${ }^{\text {Cre }}$ mice. Scale bar: $250 \mu \mathrm{m}$. (e-h) Higher magnification of E15.5 transverse TA muscle stained with (e, g) desmin and (f, h) slow myosin antibody. Slow myosin pattern was relatively similar in both (h) mutant and (f) control mice. Bold lines demarcate TA muscle and dotted lines indicate border of hindlimb. Scale bar: $100 \mu \mathrm{m}$. (i-1) Transverse distal hindlimb section showing E16.5 (i, k) general and ( $\mathrm{j}, \mathrm{l})$ slow muscle pattern in (i-j) control and (k-1) R26fxTeNT $x$ Olig $2^{\text {Cre }}$ mice. Scale bar: $250 \mu \mathrm{m}$. (m-p) Transverse distal hindlimb section showing E18.5 (m, o) general and (n, p) slow muscle pattern in (m-n) control and (o-p) R26 fxTeNT;Olig2 ${ }^{\text {Cre }}$ mice. Scale bar: $250 \mu \mathrm{m}$. 
Also, at all stages examined (E15.5, E16.5 and E18.5) (Figure 3.18 a-p), an overview of transverse hindlimb sections revealed a reduction in general muscle mass only at E18.5 (Figure $3.18 \mathrm{~m}-\mathrm{p}$ ). Examination at higher magnification of TA muscle did not reveal any altered patterns of slow myosin distribution in mutant mice. Taken

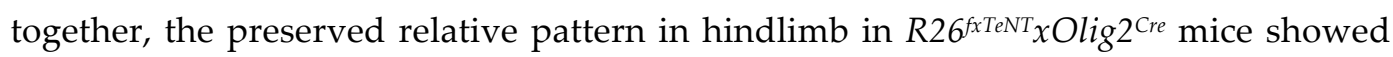
that paralysis from perturbed neurotransmission did not seem to impact primary myotube specification or formation.

\subsubsection{Neurotransmission blockade does not abolish primary and secondary formation in $\mathrm{R}^{2} 6^{f \mathrm{TeNT}} x \mathrm{Olig} 2^{\mathrm{Cre}}$ muscles}

Examination of transverse distal hindlimb sections from E18.5 TA and S muscle revealed that $\mathrm{EdU}^{+}$nuclei were present at an appreciable number within muscle fibers

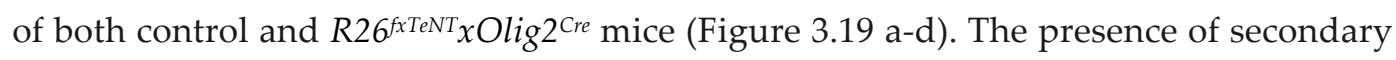
myotubes in paralyzed muscles was confirmed by EdU-labeling of myotubes with centralized nuclei (Figure $3.19 \mathrm{c}-\mathrm{d}$ ), which did not appear to be satellite cells. Additionally, absolute muscle mass of $R 26^{\text {fxTeNT}} x \mathrm{Olig} 2^{\mathrm{Cre}}$ hindlimbs increased as development progresses, thereby indicating that secondary myotubes were formed and added to the muscle bulk to contribute to muscle mass increase. Taken together, I have provided evidence that secondary myotubes are generated even in the absence of nerve activity despite claims from researchers that secondary myogenesis was profoundly inhibited following d-tbc administration (McLennan, 1983b; Sohal and Sickles, 1986). Secondary myotubes were also affected by paralysis, but not as severely as by wholesale motor neuron ablation, as determined by lesser muscle mass lost after primary myogenesis in $\mathrm{R}_{2} 6^{f \mathrm{TeNT}} \mathrm{XOlig} 2^{\mathrm{Cre}}$ mice. 


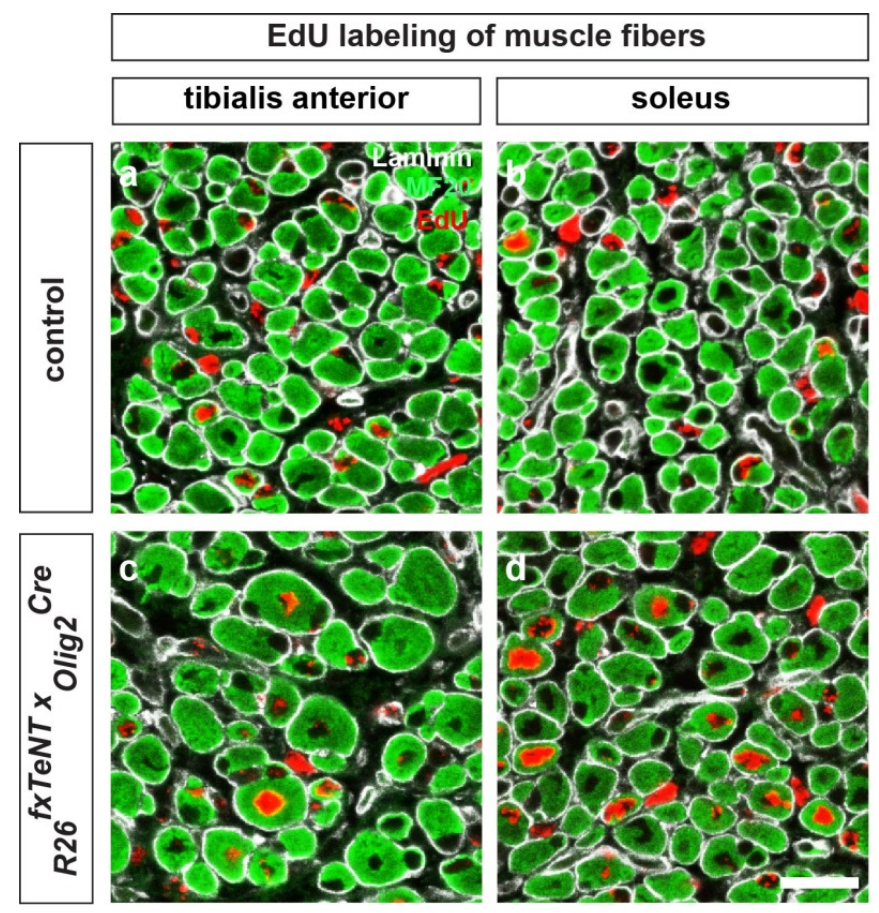

Figure 3.19: Secondary myogenesis occurs in paralyzed muscles.

(a-d) Co-immunohistochemistry on E18.5 transverse distal hindlimb sections of (a, c) TA and (b, d) S muscle from (a-b) control and (c-d) R26 fxTeNT $x$ Olig2 ${ }^{\text {Cre }}$ mice. Nuclei of muscle fibers generated during secondary myogenesis were incorporated with EdU. Secondary muscle fibers were detected in muscles of both control and mutant mice. Scale bar: $20 \mu \mathrm{m}$.

To determine if there was a compensatory response in the number of EdU-labeled cells after blocking neural activity, as is the case for $\mathrm{R}_{2} 6^{f x D T A} x \mathrm{Olig} 2^{\mathrm{Cre}}$ mice after ablating motor neurons, quantification for the number of EdU+ nuclei present within muscles of control and $R 26^{f x T e N T} x O l i g 2^{\text {Cre }}$ mice must be performed in parallel with Pax $7^{+}$ satellite cell quantification. Interestingly, the CSA of muscle fibers qualitatively appeared to be slightly enlarged in mutant fibers, although the incidence of mature fibers with perinuclei was higher in muscles of $R 26^{f x T e N T} x O l i g 2^{\text {Cre }}$ mice than $R 26^{f x D T A} x O \operatorname{Oig} 2^{\text {Cre }}$ mice. 


\subsubsection{Blocking neurotransmission results in an increase of motor neurons}

Analysis of transverse lumbar spinal cord sections of mutant mice at E18.5 revealed an evident increase in the number of motor neurons (Figure $3.15 \mathrm{~h}$ and j). To further investigate this, transverse thoracic spinal cords of E18.5 mice were also analyzed to rule out that any increase/alteration in motor neurons was specific to a certain level of the spinal cord. Indeed, thoracic motor neurons were also found in higher numbers within mutant spinal cord (Figure $3.20 \mathrm{c}-\mathrm{d}$ ) as compared to controls (Figure $3.20 \mathrm{a}-\mathrm{b}$ ).

To assess if blocking neurotransmission would alter projections to target muscles, the diaphragm, a relatively thin muscle, was examined using whole mount immunostaining. Control E18.5 diaphragm showed clustering of AChRs located postsynaptically apposed with secondary branches arising from the central branch of the phrenic nerve (Figure 3.21 a). Contrastingly, although the main trunk of the phrenic nerve formed in diaphragm of $R 26^{f x T e N T} x O \operatorname{Oig} 2^{C r e}$ mice, there was a dramatic excess of secondary and tertiary branching, with a substantial fraction of muscle's width covered by the nerve in mutants than in controls (Figure $3.21 \mathrm{~b}$ ).

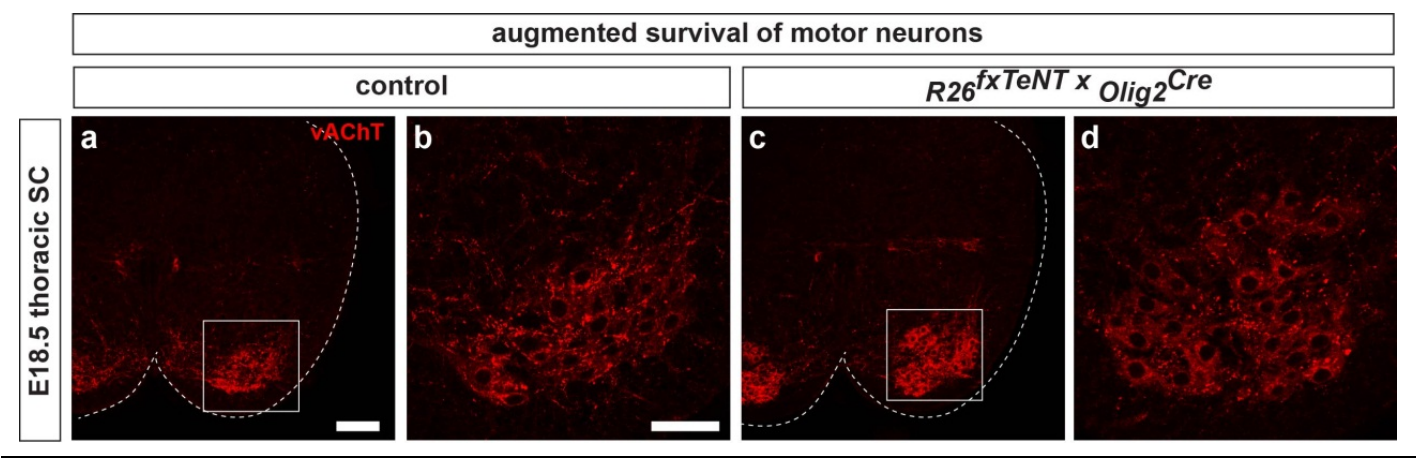

Figure 3.20: Conditional expression of TeNT augments motor neuron survival.

(a-d) E18.5 transverse thoracic spinal cord sections of (a-b) control and (c-d) R26 frTeNT $x$ Olig2 ${ }^{\text {Cre }}$ mice. (b, d) Boxed area of thoracic spinal cord at higher magnification. vAChT labeling revealed increased motor neuron numbers within spinal cord of mutant mice. Dotted lines indicate boundaries of spinal cord. a and c, scale bar: $50 \mu \mathrm{m}$; b and d, scale bar: $20 \mu \mathrm{m}$. 
AChRs in mutants were formed, but localized to a narrower endplate width and seemed to be closely associated with the primary nerve branch. Moreover, the aberrant branching pattern in mutants was confined to the intramuscular portions of the nerve, suggestive of faulty nerve-muscle interactions. Taken together, the increase in motor neuron numbers after abolishing vesicular release can be justified either by 1) the increased proliferative ability of motor neuron progenitors and 2) the augmented survival of motor neurons caused by reduction in cell elimination during the programmed cell death period. The latter hypothesis is more probable as it is supported by results from Oppenheim and colleagues (Oppenheim, 1986; Oppenheim et al., 1986; Oppenheim, 1991) who observed that blocking neural activity by using $\alpha$ BTX and d-tbc led to an increase in motor neuron numbers, presumably by the loss of activity-dependent competition between motor neuron axons while establishing synaptic connectivity with their target muscles.

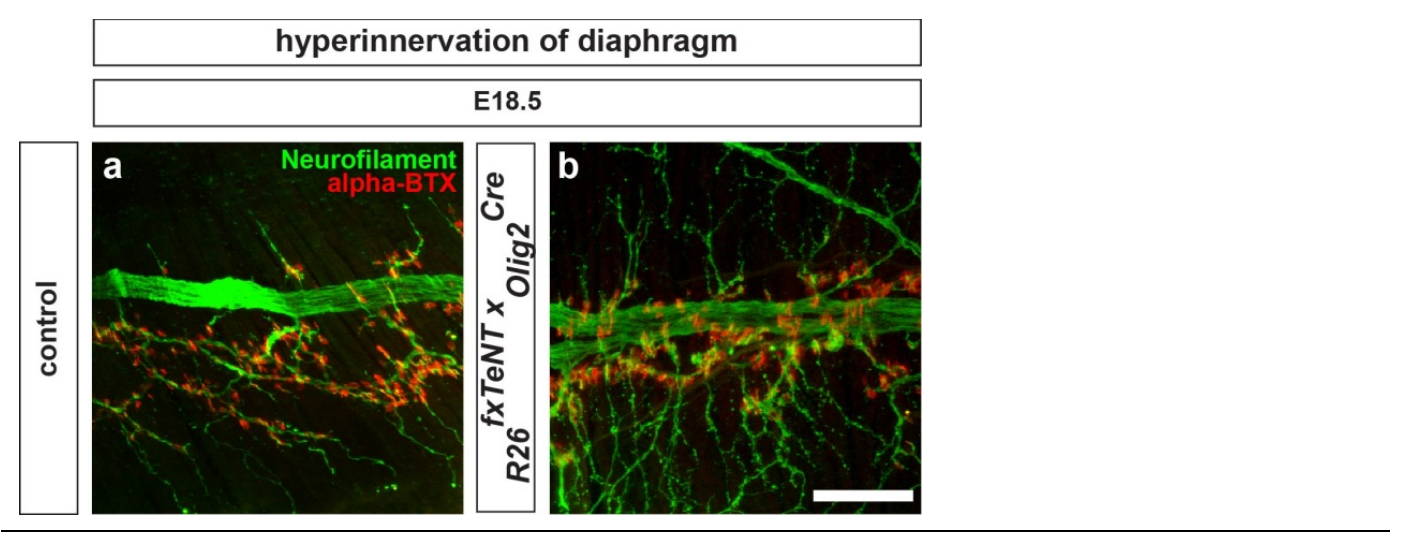

Figure 3.21: Blocking neurotransmission results in hyperinnervation of target muscles.

(a) Central branch of phrenic nerve innervating the E18.5 diaphragm was identified with neurofilament whereas postsynaptic acetylcholine receptors (AChRs) were labeled with fluorescence-conjugated $\alpha$ bungarotoxin ( $\alpha$-BTX). AChRs were apposed to secondary branches derived from the main branch of the phrenic nerve. (b) Diaphragm muscles of $R 26^{f x T e N T} x O l i g 2^{C r e}$ mice exhibited an increase in secondary and tertiary nerve branches. AChRs were misaligned to a narrower endplate. Neurofilament, pan axonal marker; $\alpha$-BTX, postsynaptic AChR cluster marker. Scale bar: $20 \mu \mathrm{m}$. 


\subsubsection{Inactivating the Chat gene results in reduced muscle mass}

Muscle mass after neurotransmission blockade in $R 26^{f x T N T} x O \operatorname{lig} 2^{\text {Cre }}$ mice was not as severely affected as $R 26^{f x D T A} x$ Olig $2^{C r e}$ mice, and was observed to be lost at later stages beyond E15.5. This discrepancy could reflect myogenic requirements for other nervecomponents besides neural activity or that TeNT expression in the $26^{\text {fxTeNT}} x \mathrm{Olig} 2^{\mathrm{Cre}}$ mice did not completely block all aspects of motor neurotransmission. Consistent with the latter hypothesis, spontaneous action potential-independent neurotransmission appears to utilize different vesicle release machinery that does not stringently require the association of synaptobrevin to the plasma membrane for vesicular fusion (Deitcher et al., 1998; Schoch et al., 2001). Therefore, it cannot be ruled out that remnant spontaneous neural activity might still prevail in $R 26^{f x T e N T} x O \operatorname{lig} 2^{C r e}$ mice, thus resulting in the observed milder impact on myogenesis compared to wholesale motor neuron ablation in $\mathrm{R}_{2} 6^{f x D T A} x \mathrm{Olig} 2^{\text {Cre }}$ mice. To address this, mice deficient for Chat (a gene encoding the enzyme choline acetyltransferase that is responsible for $\mathrm{ACh}$ biosynthesis) which have been extensively used in the study of neuromuscular junction formation were morphometrically analyzed. They displayed normal gross morphology with kyposis-like posture, hyperinnervation of muscles and they die perinatally (Misgeld et al., 2002; Brandon et al., 2003). More importantly, both spontaneous and evoked nerve activity is completely abolished in Chat mutants (Brandon et al., 2003).

Cross-examination of hindlimbs revealed that skeletal muscle mass was severely reduced in the Chat-deficient embryos, as compared to the control embryos (Figure 3.22 a-b). Strikingly, quantification of remaining muscle mass in the hindlimb of Chat mutants and their age-matched controls at E14.5 and E18.5 showed a degree of muscle reduction closely similar to $R 26^{f x D T A} x \mathrm{Olig} 2^{\mathrm{Cre}}$ mice. The onset of muscle loss in Chat deficient embryos was detected at E14.5 with a 13\% reduction and at E18.5, remaining was $44 \%$ of control mice (Figure 3.22 c). Correspondingly, apoptotic cells were found 
at a significantly higher number in transverse distal hindlimb sections of mutant mice at both stages analyzed (Figure $3.22 \mathrm{~d}$ ).

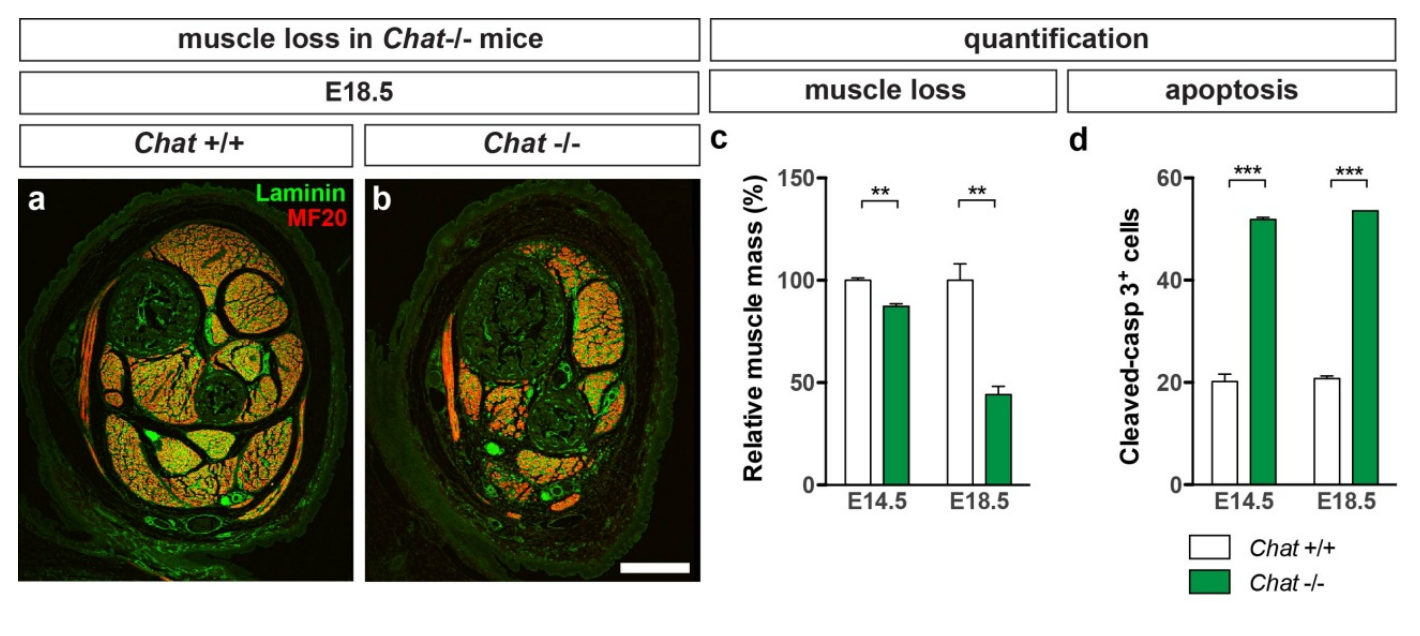

Figure 3.22: Deleting the Chat gene has severe impacts on myogenesis.

(a-b) E18.5 transverse distal hindlimb sections of (a) control and (b) Chat-/- mice. Mutant muscles were drastically reduced when compared to controls. Akin to what was observed before with $R 26^{f x D T A} x \mathrm{Olig} 2 \mathrm{Cre}$ and $R 26^{f x T e N T} x O l i g 2^{C r e}$ mice, some groups of muscles were absent whereas others were merely reduced. Scale bar: $250 \mu \mathrm{m}$. (c) Quantification of muscle mass remaining in the hindlimb of control and Chat-/- mice at E14.5 and E18.5 revealed a similar pattern of progressive muscle loss that occurred in $R 26^{f x D T A} x \mathrm{Olig} 2^{\mathrm{Cre}}$ mice. (d) Quantification of apoptotic cells within E14.5 and E18.5 muscles of control and mutant mice. At least three sections from each of three control and mutant embryos were counted for each time point. Data are presented as mean \pm SEM (two-tailed Student's $t$ test with unequal variance, $n=3$ per genotype, per stage). ${ }^{* * *}, \mathrm{p}<0.001 ;{ }^{* *}, \mathrm{p}<0.01 ;{ }^{*}, \mathrm{p}<0.05$.

At E14.5, the number of apoptotic cells in mutants was around 52 per section as compared to about 20 per section in controls. At E18.5, the average number of apoptotic cells remained at around 54 per section for mutant mice in contrast to 21 per section in control mice. A likely increase and progressive decline in the number of apoptotic cells as observed in the previous mouse lines could occur in between the stages analyzed (E14.5 and E18.5). Conditional deletion of Chat, which resulted in the abolishment of both spontaneous and evoked neurotransmission, exacerbated the severity of muscle loss in comparison to only blocking vesicular release in 
R26 ${ }^{f x T N T} x$ Olig2 $2^{\text {Cre }}$ mice. Furthermore, the extent of muscle reduction in Chat mutants was comparable to $R 26^{f x D T A} x O l i g 2^{C r e}$ mice with complete motor neuron removal. Herein, based on the assumption that there is residual spontaneous vesicular release in $R 26^{f x T e N T} x O l i g 2^{C r e}$ mice, that two conclusions can be made: (1) spontaneous nerve activity is critical for myogenesis during early development, and evoked nerve activity becomes increasingly important further into development; (2) ACh is the central nerve-derived molecule which could act with other factors to relay nerve activity into signals to activate expression of both muscle-specific and muscle-associated genes.

\subsubsection{Blocked evoked neurotransmission did not disrupt sarcomere formation or organization}

I have shown in Figure 3.13 that sarcomere structures were disrupted and indistinct in $R 26^{f x D T A} x$ Olig $2^{\text {Cre }}$ mice after motor neuron removal. However, it was yet unclear if promotion of myofibrillogenesis (the formation of sarcomeres) is driven by motor neuron activity, trophic factors released from motor nerve, or simply by signals transduced by molecules activated via nerve-muscle contact. To narrow down the possibilities, E18.5 longitudinal muscle fibers were co-immunostained with MF20 and desmin to visualize probable alteration to sarcomere pattern in skeletal muscles of $R 26^{f x T e N T} x$ Olig2 ${ }^{\text {Cre }}$ mice. The striated pattern arising from units of sarcomeres was surprisingly well preserved in mutant muscle fibers, having a similar appearance to control fibers (Figure $3.23 \mathrm{a}-\mathrm{b}$ ), a stark contrast to $\mathrm{R} 26^{f \mathrm{fTeNT}} x \mathrm{Olig} 2^{\mathrm{Cre}}$ mice.

Gene analysis demonstrated no overt disparity in the expression of sarcomereassociated genes, regardless of the localization of the proteins they encode to the different components of the sarcomere. With the exception of 7 genes (sorted according to $\geq 1.5$ fold-change, $p$-value $\leq 0.05$, expression level $\geq 100$ ), no significant disparity could be distinguished between muscles of control and mutant mice. The 
normalized expression value of sarcomere-associated genes in control and $R 26^{f x T e N T} x O l i g 2^{\text {Cre }}$ mice, as well as the statistical analysis can be found under Table 3.2.

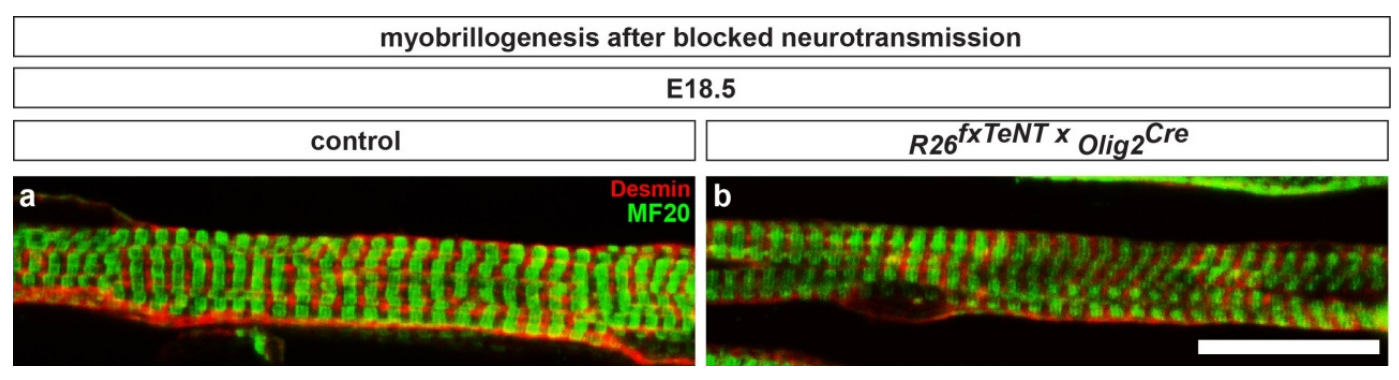

Figure 3.23: Striated muscle pattern is intact in paralyzed muscles.

(a-b) Co-immunostaining of control E18.5 (a) control and (b) R26fxTeNT $x$ Olig2 Cre longitudinal muscle fiber with desmin and MF20 antibodies revealed a striated pattern with well-aligned sarcomeres. Sarcomere integrity appeared well preserved in mutant fibers. Scale bar: $20 \mu \mathrm{m}$.

This finding therefore suggests that nerve activity is dispensable in the differentiation process to allow muscle fibers to become functionally contractile, assuming that both spontaneous and evoked neural activity is completely eliminated in $\mathrm{R} 26^{\mathrm{fxTNNT}} x \mathrm{Olig} 2^{\mathrm{Cre}}$ mice. Alternatively, and more likely, if there is remnant spontaneous activity in mutants, it can be concluded that evoked nerve activity is not required in the differentiation process to allow muscle fibers to become functionally contractile. Rather, nerve contact, spontaneous nerve activity, or nerve-derived factors could act as critical determinants in promoting myobrillogenesis as part of the terminal differentiation program to form functionally contractile muscle fibers. 


\begin{tabular}{|c|c|c|c|c|c|}
\hline Component & Gene & Control & Mutant & Fold-change & $P$-value \\
\hline \multirow{16}{*}{ Z-line } & Actn2 & 3369.28 & 3192.73 & 1.06 & $\mathrm{~ns}$ \\
\hline & Actn3 & 106.43 & 87.13 & 1.22 & ns \\
\hline & Cmya1 & 31.68 & 21.85 & 1.45 & * \\
\hline & Csrp3 & 1453.40 & 899.13 & 1.62 & * \\
\hline & $L d b 3$ & 56.75 & 34.65 & 1.64 & * \\
\hline & Myot & 75.43 & 56.40 & 1.34 & ns \\
\hline & Myoz1 & 155.18 & 90.93 & 1.71 & * \\
\hline & Myoz2 2 & 862.30 & 650.88 & 1.32 & ns \\
\hline & Obscn & -5.43 & -6.45 & 1.19 & $\mathrm{~ns}$ \\
\hline & Pak1 & 30.23 & 35.40 & 1.17 & ns \\
\hline & Pdlim3 & 1433.45 & 1153.65 & 1.24 & ns \\
\hline & Рpp3са & 395.73 & 423.60 & 1.07 & ns \\
\hline & Synpo2 & -4.20 & -0.40 & 10.50 & ns \\
\hline & Synpo2l & 61.95 & 76.15 & 1.23 & ns \\
\hline & Tcap & 289.38 & 232.43 & 1.25 & ns \\
\hline & $Z y x$ & 8178.23 & 7553.40 & 1.08 & $\mathrm{~ns}$ \\
\hline \multirow{9}{*}{ A-band } & Myh2 & 8.68 & 13.38 & 1.54 & $\mathrm{~ns}$ \\
\hline & Myh4 & -11.03 & -8.65 & 1.27 & ns \\
\hline & Myh6 & 8.98 & 15.10 & 1.68 & ns \\
\hline & Myh7 & 31.83 & 20.05 & 1.59 & ns \\
\hline & Myh8 & 14096.78 & 12783.68 & 1.10 & ns \\
\hline & Myl1 & 14875.75 & 13460.05 & 1.11 & ns \\
\hline & Myl2 & 732.10 & 316.95 & 2.31 & * \\
\hline & Myl3 & 1408.55 & 754.75 & 1.87 & ** \\
\hline & Myl4 & 21288.53 & 18582.98 & 1.15 & * \\
\hline \multirow{6}{*}{ Third filament } & Capn3 & 19.28 & 18.73 & 1.03 & ns \\
\hline & Hspb1 & 2384.425 & 1929.15 & 1.24 & ns \\
\hline & Mybphl & 0.58 & 1.25 & 2.17 & ns \\
\hline & Mypn & 23.15 & 19.13 & 1.21 & ns \\
\hline & Rnf34 & 389.10 & 394.93 & 1.01 & $\mathrm{~ns}$ \\
\hline & $T t n$ & 2367.75 & 1910.08 & 1.24 & ns \\
\hline \multirow{11}{*}{ M-line } & Eno3 & 2299.73 & 1865.50 & 1.23 & ns \\
\hline & Calm2 & 1520.80 & 1625.88 & 1.07 & ns \\
\hline & Calm3 & 5319.78 & 5524.60 & 1.04 & ns \\
\hline & Calm4 & 2165.08 & 1393.13 & 1.55 & ns \\
\hline & Calm/3 & 101.73 & 66.68 & 1.53 & * \\
\hline & Ckm & 770.08 & 413.95 & 1.86 & * \\
\hline & Ckmt2 & 82.15 & 42.45 & 1.94 & ns \\
\hline & Myom1 & 328.30 & 294.20 & 1.12 & ns \\
\hline & Myom 2 & -3.60 & -8.13 & 2.26 & ns \\
\hline & Smpx & 116.75 & 107.13 & 1.09 & ns \\
\hline & Trim63 & 57.30 & 49.63 & 1.15 & ns \\
\hline \multirow{15}{*}{ I-band } & Acta2 & 6252.18 & 7054.70 & 1.13 & ns \\
\hline & Acta1 & 16451.45 & 13269.65 & 1.24 & ns \\
\hline & Actc1 & 9158.68 & 7818.75 & 1.17 & ns \\
\hline & $\mathrm{Nebl}$ & -0.48 & -6.33 & 13.32 & * \\
\hline & Tmod1 & 334.30 & 271.55 & 1.23 & ns \\
\hline & Tmod2 2 & 56.35 & 42.98 & 1.31 & ns \\
\hline & Tmod3 & 228.60 & 213.20 & 1.07 & ns \\
\hline & Tmod4 4 & 218.40 & 128.65 & 1.70 & $*$ \\
\hline & Tnnc1 & 15379.73 & 15886.05 & 1.03 & ns \\
\hline & Tnnc2 & 13501.33 & 12020.15 & 1.12 & ns \\
\hline & Tnni1 & 597.43 & 596.73 & 1.00 & ns \\
\hline & Tnni2 & 5287.88 & 4104.28 & 1.29 & ns \\
\hline & Tnnt1 & 9539.23 & 8268.45 & 1.15 & ns \\
\hline & Tnnt3 & 2154.00 & 1532.85 & 1.41 & ns \\
\hline & Tpm2 & 2393.45 & 2051.53 & 1.17 & ns \\
\hline \multirow{9}{*}{ Intermediate filament } & Cryab & 969.98 & 965.28 & 100 & ns \\
\hline & Des & 1599.23 & 1360.20 & 1.18 & ns \\
\hline & $D m n$ & 20.73 & 21.60 & 1.04 & ns \\
\hline & Mpp4 & 2.75 & 5.53 & 2.01 & ns \\
\hline & Nes & 398.45 & 452.80 & 1.14 & ns \\
\hline & Plec1 & 218.10 & 193.23 & 1.13 & ns \\
\hline & Sync & -9.65 & -7.70 & 1.25 & ns \\
\hline & $\mathrm{Vcl}$ & 1348.08 & 1175.48 & 1.15 & ns \\
\hline & Vim & 4284.40 & 4432.43 & 1.03 & ns \\
\hline
\end{tabular}

Table 3.2: List of sarcomere-associated genes in control and $\mathrm{R}^{2} \mathrm{G}^{f \mathrm{xTeNT}} x \mathrm{Olig} 2^{\mathrm{Cre}}$ mice.

Relative units of gene expression are shown under 'control' and 'mutant' field. Data under 'control' and 'mutant' field are presented as mean relative units of gene expression. (One-tailed Student's $t$ test with unequal variance, $\mathrm{n}=4$ per genotype). ${ }^{* *}, \mathrm{p}<0.001 ;{ }^{* *}, \mathrm{p}<0.01 ;{ }^{*}, \mathrm{p}<0.05$. Red, upregulated genes $\geq 1.0$ fold. 


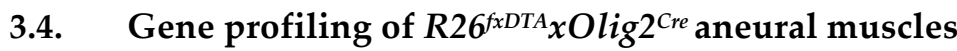

\subsubsection{Differential gene expression underlying myogenesis in the absence of motor neurons}

In the adult, denervation is mainly mediated by ubiquitin-proteolysis via the proteasome, with the end result being skeletal muscle atrophy. Additionally, denervation-induced apoptosis, also implicated during adult denervation, also contributes to muscle mass reduction that occurs around 2 weeks following denervation (O'Leary et al., 2012). In contrast, muscle loss in the R26fxDTAxOlig2 ${ }^{\mathrm{Cre}}$ embryo spans a course of merely 5 days (from E14.5-E18.5) before birth when muscle reduction is most severe. Upon embryonic denervation, muscles failed to undergo terminal differentiation and were rapidly lost in $R 26^{f x D T A} x O \operatorname{lig} 2^{C r e}$ mice via apoptosis. The altered structural muscle properties in these aneural mice could already be detected by the lack of sarcomeres at E14.5 (data not shown). My results indicate that the time course for the activation of denervation-induced apoptotic signaling pathway resulting in muscle mass reduction progresses faster in the embryos, and the structural muscle properties that are altered after denervation are also fundamentally different from that observed after adult denervation. These findings, taken together, imply different requirements of motor nerve input for embryonic myogenesis on the one hand and maintenance of adult muscle on the other.

While molecular pathways implicated in the muscle atrophy resulting from denervation in the adult are rapidly emerging (Bodine et al., 2001; Moresi et al., 2010; Macpherson et al., 2011; O'Leary et al., 2012), little is known about the pathways and mechanisms involved in the response of embryonic muscle towards denervation. To address this, I have thus performed RNA sequencing and gene microarray experiments using whole hindlimb musculature isolated from E14.5 and E15.5 control and $R 26^{f x D T A} x O \operatorname{lig} 2^{C r e}$ mice, respectively, in order to identify in an unbiased manner possible innervation-dependent transcriptional programs. Heatmaps were used to 
represent the transcriptome data which revealed 195 differentially downregulated genes (Figure 3.24) and 218 upregulated genes (Figure 3.25) which were sorted according to the following criteria: $\geq 1.5$ fold-change, $p$-value $\leq 0.05$ and expression level $\geq 100$. Expression of housekeeping genes such as Gapdh (glyceraldehyde-3phosphate 7l) and Tuba1b (tubulin, $\alpha 1 \mathrm{~B}$ ) in control and mutant muscles showed no significant difference.

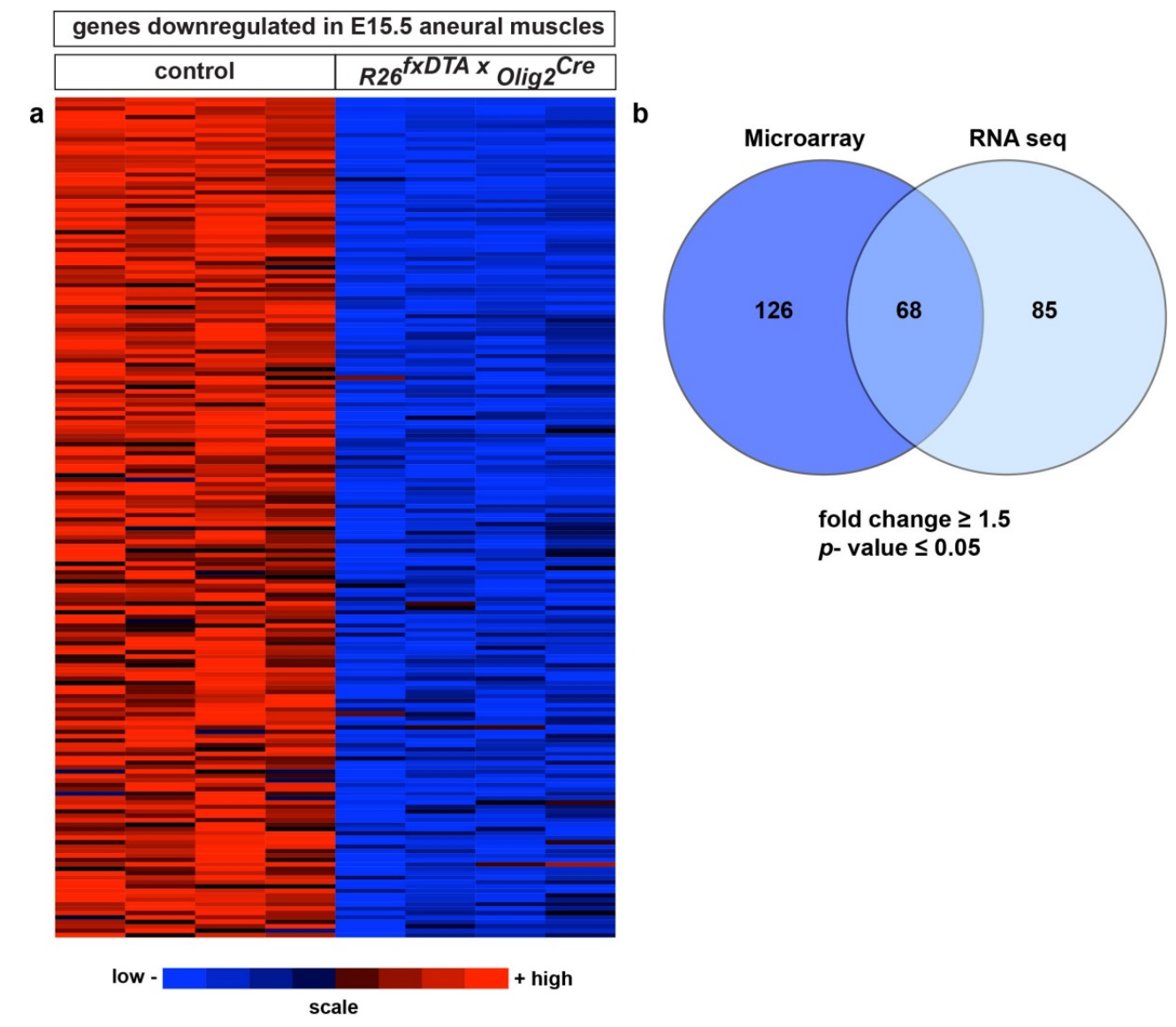

Figure 3.24: Downregulated gene profiles in $\mathrm{R}_{2} 6^{\text {fxTA }} x \mathrm{Olig} 2^{\mathrm{Cre}}$ muscles.

(a) Heatmap representation of downregulated genes following microarray analysis in E15.5 muscles of $R 26^{f x D T A} x$ Olig2 ${ }^{\text {Cre }}$ mice. The threshold level for gene expression was set to $\geq 100$ relative units. (b) Venn diagram showing overlap of 68 differentially downregulated genes obtained from muscles of mutant mice following microarray processing (left, total 194 genes) and RNA sequencing (right, total 153 genes). The genes were sorted accordingly to the following criteria: $\geq 1.5$ fold-change and $p$-value $\leq 0.05$. Red, high level of expression; Blue, low level of expression. 


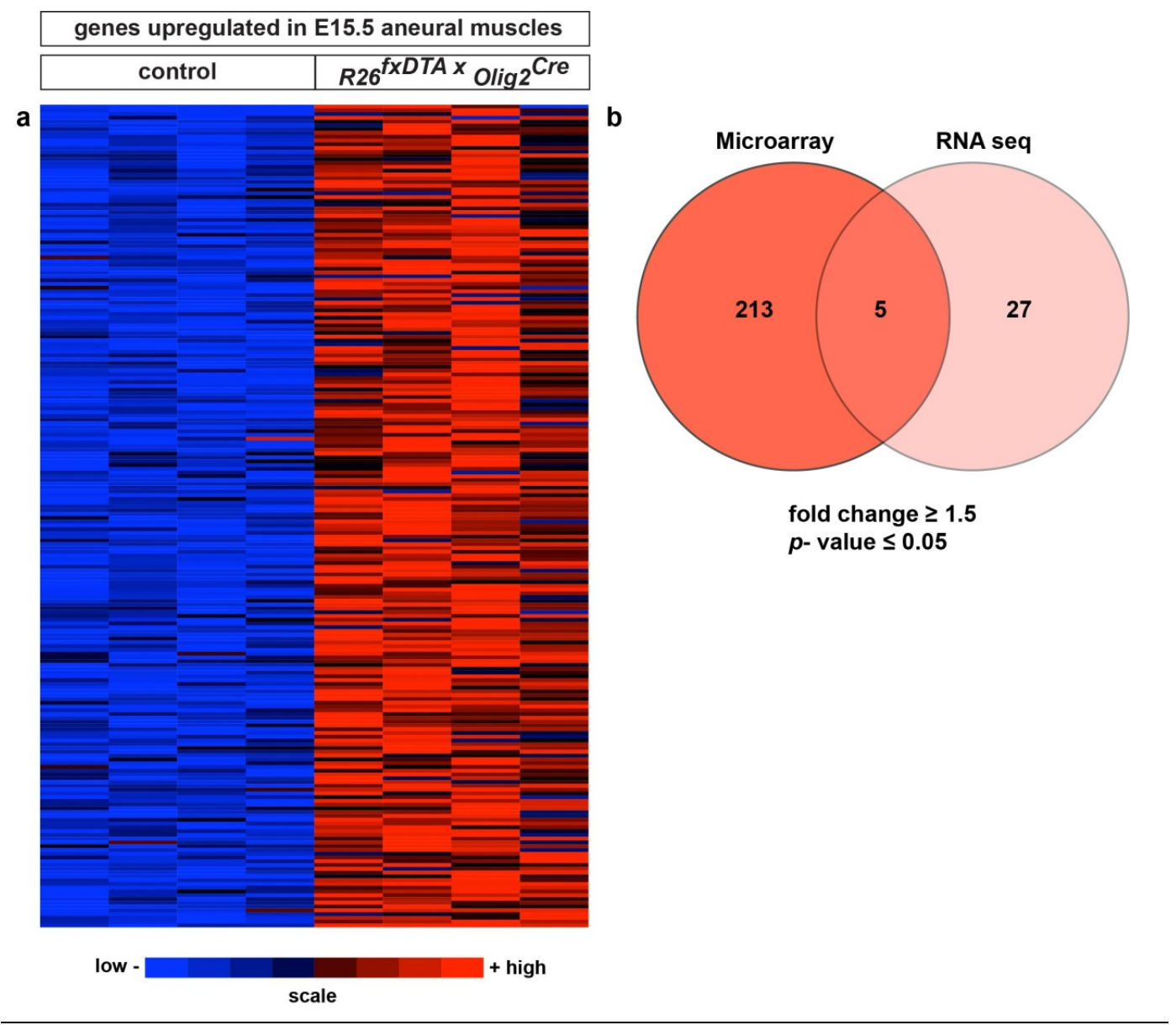

Figure 3.25: Heatmap of upregulated genes in $\mathrm{R}^{26^{f x D T A}} x \mathrm{Olig} 2^{\mathrm{Cre}}$ muscles.

(a) Heatmap representation of upregulated genes following microarray analysis in E15.5 muscles of R26fxDTA $x$ Olig2Cre mice. The threshold level for gene expression was set to $\geq 100$ relative units. (b) Venn diagram showing overlap of 5 differentially upregulated genes obtained from muscles of mutant mice following microarray processing (left, total 218 genes) and RNA sequencing (right, total 32 genes). The genes were sorted accordingly to the following criteria: $\geq 1.5$ fold-change and $p$-value $\leq 0.05$. Red, high level of expression; Blue, low level of expression.

RNA sequencing data revealed 153 downregulated and 32 upregulated genes, of which 68 downregulated genes and 5 upregulated genes overlapped (downregulated genes $32.2 \%$ overlap, upreguated genes $2.1 \%$ overlap) with differentially regulated genes obtained from the microarray experiments (Figure 3.24 and Figure 3.25). The complete set of differentially expressed genes were classified, separately for 
downregulated and upregulated genes, according to the Panther classfication system (Mi et al., 2010; Mi et al., 2012).

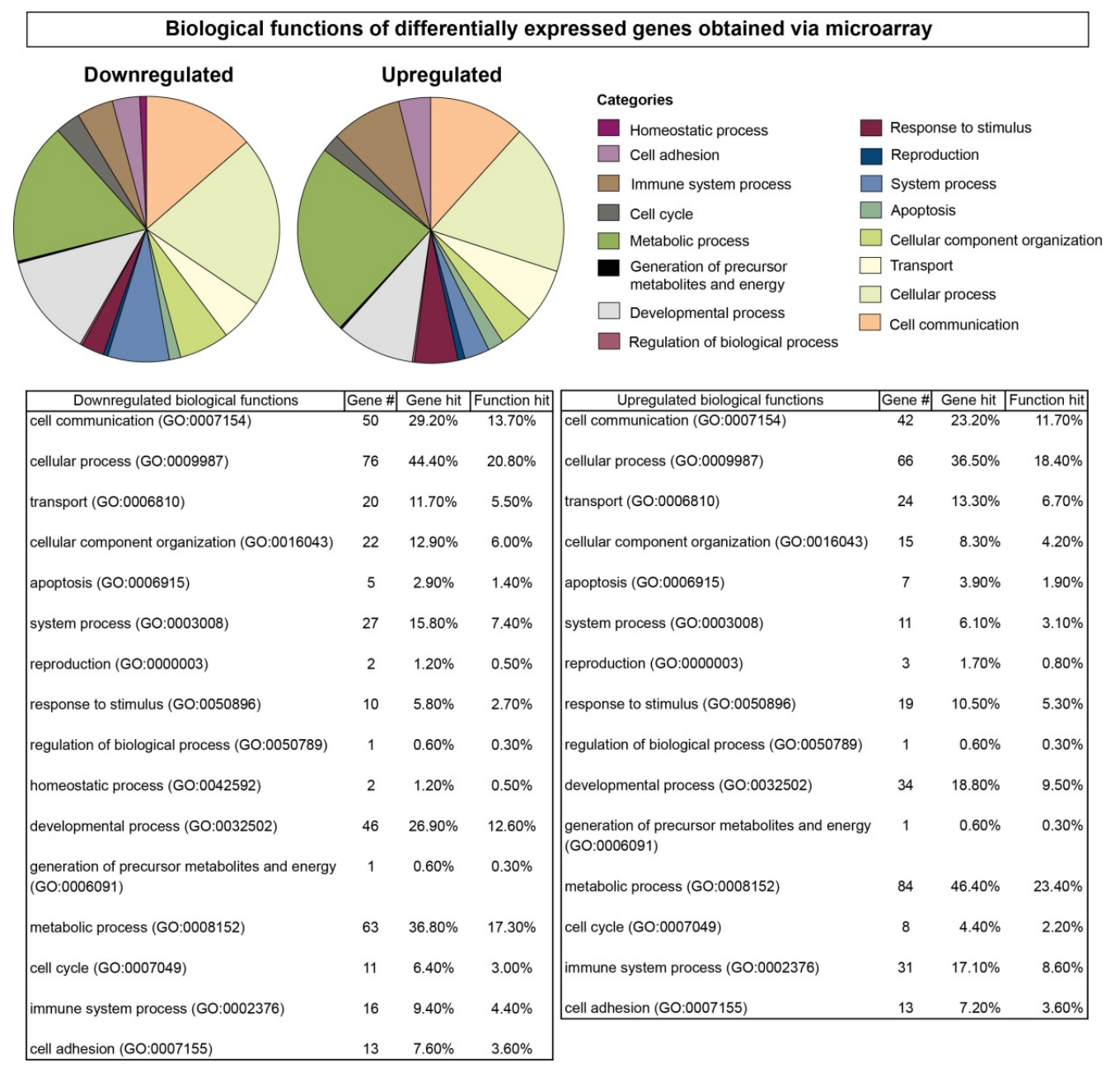

\footnotetext{
Figure 3.26: Biological function profiles of differentially expressed genes obtained via microarray. Differential expressed genes in $R 26^{f x D T A} x O l i g 2$ Cre muscles were classified based on their known biological functions (see different categories). For downregulated genes, 171 genes were recognized as the total number of gene input and a total of 200 function hits were found for these genes. For upregulated genes, 181 genes were recognized as the total number of gene input and a total of 359 function hits were found for these genes. Gene hit: percentage of input gene hit over total genes; Function hit: percent of gene hit against total function hits.
} 




\footnotetext{
Figure 3.27: Biological function profiles of differentially expressed genes obtained via RNA sequencing. Differentially expressed genes in $R 26^{f x D T A} x O \operatorname{Oig} 2^{\mathrm{Cre}}$ muscles were classified based on their known biological functions (see different categories). For downregulated genes, 135 genes were recognized as the total number of gene input and a total of 449 function hits were found for these genes. For upregulated genes, 26 genes were recognized as the total number of gene input and a total of 97 function hits were found for these genes. Gene hit: percentage of input gene hit over total genes; Function hit: percent of gene hit against total function hits.
}

The most prominently represented biological processes associated with the thus identified innervation-dependent genes were cellular, metabolic, cell communication and developmental processes (Figure 3.26 and Figure 3.27). Interestingly, DAVID analysis (Huang da et al., 2009b; Huang da et al., 2009a) demonstrated that 
differentially downregulated genes largely take part in canonical KEGG pathways relating to cardiac muscle contractibility and diseases (Table 3.3).

\begin{tabular}{|c|c|c|c|c|c|}
\hline \multicolumn{6}{|c|}{ 3.3 Downregulated canonical pathways: $g$} \\
\hline Category & Term & Count & $\%$ & $P$-value & Benjamini \\
\hline \multirow{5}{*}{ KEGG_PATHW/ } & $\begin{array}{l}\text { Arrhythmogenic right ventricular cardiomyopathy } \\
\text { (ARVC) }\end{array}$ & 7 & 4.1 & *** & ** \\
\hline & Cardiac muscle contraction & 7 & 4.1 & *** & *** \\
\hline & & & & & \\
\hline & Hypertrophic cardiomyopathy (HCM) & 6 & 3.5 & *** & * \\
\hline & Dilated cardiomyopathy & 6 & 3.5 & *** & * \\
\hline
\end{tabular}

\begin{tabular}{|c|c|c|c|c|c|}
\hline Category & Term & Count & $\%$ & $P$-value & Benjamini \\
\hline \multirow{3}{*}{ KEGG_PATHWAY } & Glutathione metabolism & 7 & 3.7 & *** & *** \\
\hline & Metabolism of xenobiotics by cytochrome P450 & 7 & 3.7 & *** & *** \\
\hline & Drug metabolism & 7 & 3.7 & *** & *** \\
\hline
\end{tabular}

\begin{tabular}{|c|c|c|c|c|c|}
\hline Category & Term & Count & $\%$ & $P$-value & Benjamini \\
\hline \multirow{5}{*}{ KEGG_PATHWAY } & Hypertrophic cardiomyopathy (HCM) & 7 & 4.7 & *** & ** \\
\hline & Dilated cardiomyopathy & 7 & 4.7 & *** & ** \\
\hline & Cardiac muscle contraction & 6 & 4.0 & *** & ** \\
\hline & Tight junction & 6 & 4.0 & ** & * \\
\hline & Focal adhesion & 7 & 47 & ** & * \\
\hline
\end{tabular}

Table 3.3: DAVID analysis of top up- or downregulated canonical pathways.

Differentially regulated genes obtained from (a) microarray profiling experiment and (b) RNA sequencing experiment were shown to largely have functions related to the signaling pathways listed above. Gene list input was selected as per the following criteria: $\geq 1.5$ fold-change and $p$-value $\leq 0.05$. The threshold level for gene expression was set to $\geq 100$ relative units. ${ }^{* *}, \mathrm{p}<0.001{ }^{* *}, \mathrm{p}<0.01 ;{ }^{*}, \mathrm{p}<0.05$.

This finding is in agreement with my conclusion that motor innervation is required to drive the terminal differentiation of skeletal myotubes into contractile fibers (direct inference since cardiac and skeletal muscles are both striated muscles and are hence comparable). Additionally, the fact that pathways associated with cardiac diseases 
such as hypertrophic cardiomyopathy ( $\mathrm{HCM})$, a disease primarily associated with myocardium hypertrophy, sarcomere disarray and mutations in sarcomeric genes, are implicated in aneural muscles of $R 26^{f x D T A} x \mathrm{Olig} 2^{\mathrm{Cre}}$ mice not only reinforces my finding

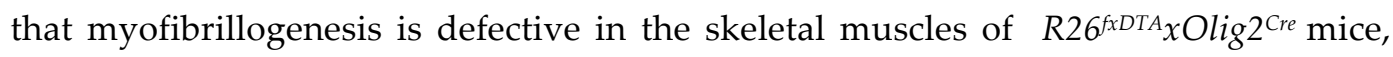
but could also account for the hypertrophy of denervated muscle fibers described in earlier sections.

\subsubsection{Potential myogenic effectors regulated by motor innervation}

Removal of motor neurons affected the expression of several genes, which were considered "candidate genes" based on their potential role(s) in translating neural activity and/or contact-dependent signals into the promotion of embryonic myogenesis. Candidate genes were selected based on a set of criteria based on significance and amplitude of altered expression levels (1.5 $\geq$ fold-change, $p$-value $\leq 0.05$ and relative expression value $\geq 100$ ), as well as their potential function in myogenesis. Since muscle mass reduction was already observed at E15.5, differential gene regulation that could be secondary consequences of motor neuron ablation had to be considered and filtered out. Thus, expression of these genes has been verified via quantitative polymerase chain reaction (qPCR) throughout three developmental stages (E13.5, E15.5 and E18.5) respectively reflecting stages before, during onset and endstage of muscle degeneration. A discrete set of genes encoding potential myogenic factors was downregulated as early as E13.5, a stage that is prior to the onset of denervation-induced muscle degeneration and after the initiation of primary myogenesis (Figure 3.28 a-d). These genes were therefore tentatively classified as "early response" genes. These include genes encoding the muscle LIM protein (Sapd) which is localized to the sarcomere and has been characterized as a positive regulator of myogenesis, transcription factor Myfd which is a member of the MRF family and is important for the terminal differentiation of myotubes during development, Snaid, a 
zinc-finger family transcriptional repressors implicated in carcinogenesis and embryogenesis and Fgfd, a fibroblast growth factor specifically accumulated in skeletal muscles during myogenesis was also downregulated.

candidate gene expression in aneural muscles throughout different developmental stages
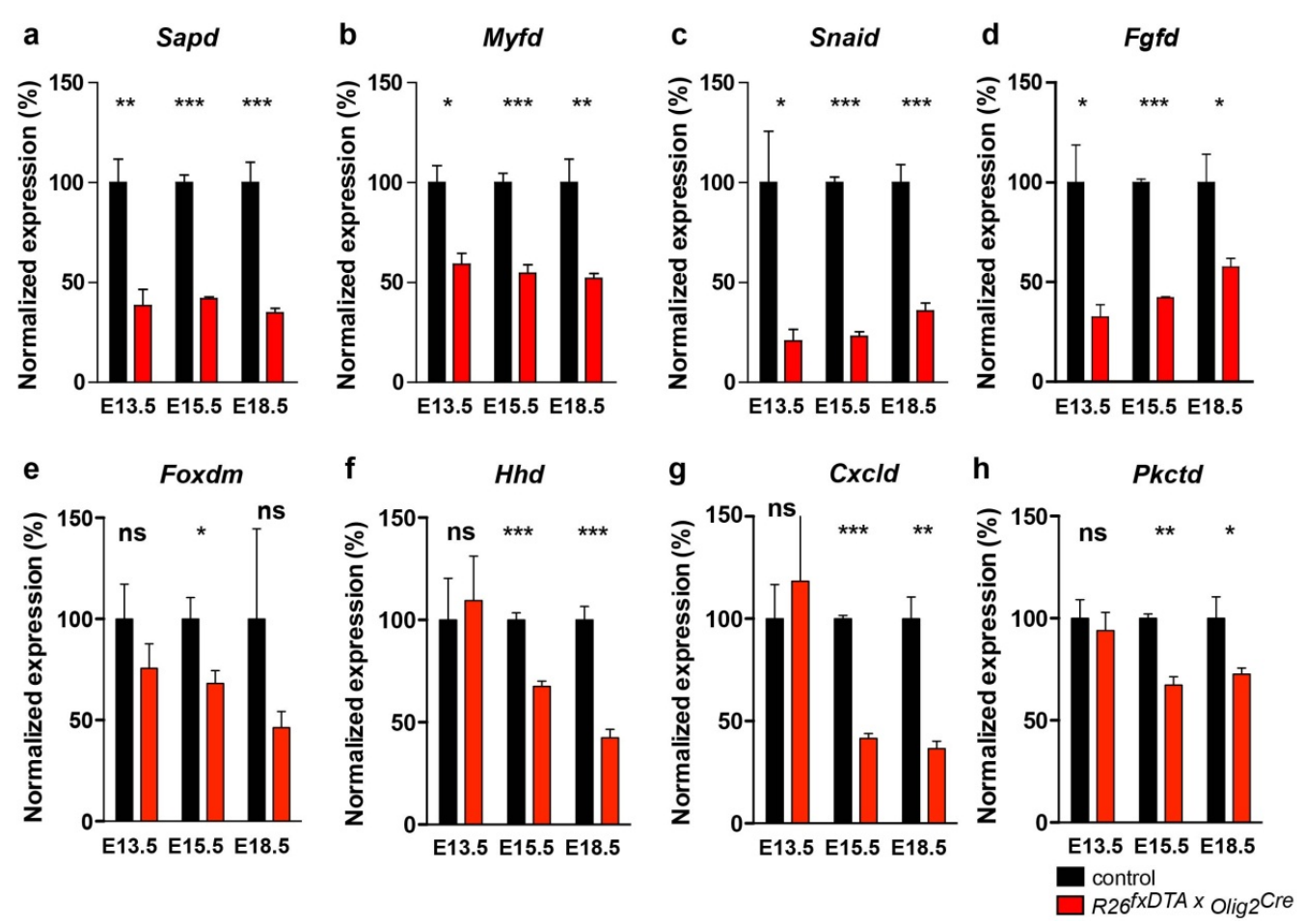

Figure 3.28: qPCR verification of downregulated candidate gene expression profiles throughout development.

(a-d) Early response genes such as (a) Sapd, (b) Myfd, (c) Snaid and (d) Fgfd were chosen as prime candidate genes because they displayed a differential response to motor neuron removal very early in development at E13.5 in muscles of $\mathrm{R}^{2} 6^{f x D T A} x \mathrm{Olig} 2 \mathrm{Cre}$ mice. (e-h) Late response genes such as (e) Foxdm, (f) Hhd, (g) Cxcld and (h) Prkctd were selected as candidate genes that showed differential downregulation only after E13.5. They are potentially downstream effectors of positively identified prime candidate genes. (Two-tailed Student's $t$ test with unequal variance, $\min n=3$ per genotype, per stage). qPCR: quantitative polymerase chain reaction. ${ }^{* *}, \mathrm{p}<0.001 ;{ }^{* *}, \mathrm{p}<0.01 ;{ }^{*}, \mathrm{p}<0.05$.

Because these genes were impacted early by motor neuron removal they were therefore considered as prime candidate effectors of motor innervation-dependent 
myogenesis. Among other downregulated candidates with potential myogenic activities, some were considered as 'late response' genes because their expression was downregulated after E15.5 and beyond but not at E13.5 (Figure 3.28 e-h). These include genes encoding Foxdm, a member of the forkhead winged/helix box (FOX) family of transcription factors which is implicated in various processes including metabolism and cell proliferation; Hhd, a member of the hedgehog family of morphogenic proteins implicated in organogenesis (Yao et al., 2002) and Cxcld, a putative member of the secreted chemokine family of proteins, which are secreted proteins and are important for the migration of muscle precursor cells during myogenesis (Vasyutina et al., 2005; Yusuf et al., 2006), as well as for macrophage infiltration of damaged muscle tissues (McLennan, 1996). Additionally, transcripts of Pkctd that encodes for 'novel' calcium-independent but phospholipid-dependent protein kinase C (Madaro et al., 2012), the PKC isoform expressed predominantly in skeletal muscles, were also negatively regulated in aneural muscles. This PKC isoform is known to take part in various muscle regenerative processes (Moraczewski et al., 2002). It is possible that the 'late response' genes are effectors of the positively identified 'early response' genes, but they may also be secondarily affected by the process of muscle degeneration (see Table 3.4 for normalized relative expression of candidate genes and statistical analysis).

\subsubsection{Muscle-associated genes affected by the removal of motor neurons}

Besides the abovementioned candidate genes (Figure 3.28), examination of the downregulated group and verification with qPCR also revealed several muscleassociated genes (Figure 3.29), including myofilament genes such as Myl2 (myosin light chain, cardiac slow isoform, data not shown), Myl3 (myosin light chain, skeletal slow isoform) and Myh3 (myosin heavy chain, embryonic isoform); members of the muscle ankyrin repeat protein (MARP) family, Ankrd1 and Ankrd2 (qPCR verification 
was only performed for Ankrd1); genes encoding muscle-specific metabolic proteins $\mathrm{Ckm}$ and Eno3; gene encoding the transcription factor Fhl1 which is upregulated during embryonic skeletal muscle development (Loughna et al., 2000) and is implicated in the modulation of muscle mass (Cowling et al., 2008); muscle spindle specific gene Etv4; and orphan nuclear receptor gene Esrrb (Figure 3.29). In the mouse, Myh3 is expressed in embryonic muscles whereas Myh8, the perinatal isoform of myosin heavy chain is found in fetal muscles. Microarray and qPCR results showed that Myh8 expression was unaltered in muscles of $R 26^{f x D T A} x \mathrm{Olig} 2^{\mathrm{Cre}}$ mice at E15.5 or all developmental stages investigated, respectively. Myh8 was nevertheless included in the analysis as a comparison to Myh3 expression which is associated with the initiation of sarcomere formation (Karsch-Mizrachi et al., 1989). The downregulation of Ankrd1, a gene that promotes hypertrophy implies that the increase in muscle fiber CSA in Figure 3.6 was less likely to be attributed to bona fide hypertrophy, but could be a sign of degeneration. Intriguingly, the drastic reduction in the expression of Myl3 was observed already at E13.5, just one day after muscle mass cleavage completion and the formation of first myotubes. The early response of Myl2 and Myl3 seems to indicate that motor innervation plays a crucial role in promoting the expression of structural genes. Under normal conditions, the expression of muscle creatine kinase $(\mathrm{Ckm})$, forkhead LIM-domain protein (Fhl1) and beta enolase (Eno3) is the defining feature of the maturation of skeletal muscles during myogenesis (Fougerousse et al., 2001; Cowling et al., 2008; Messina et al., 2010). Together with the failure to induce sarcomeric gene expression, the reduced expression of these genes in mutant $R 26^{f x D T A} x O \operatorname{Oig} 2^{\text {Cre }}$ muscles strongly suggests a block in myofiber terminal differentiation and maturation. 


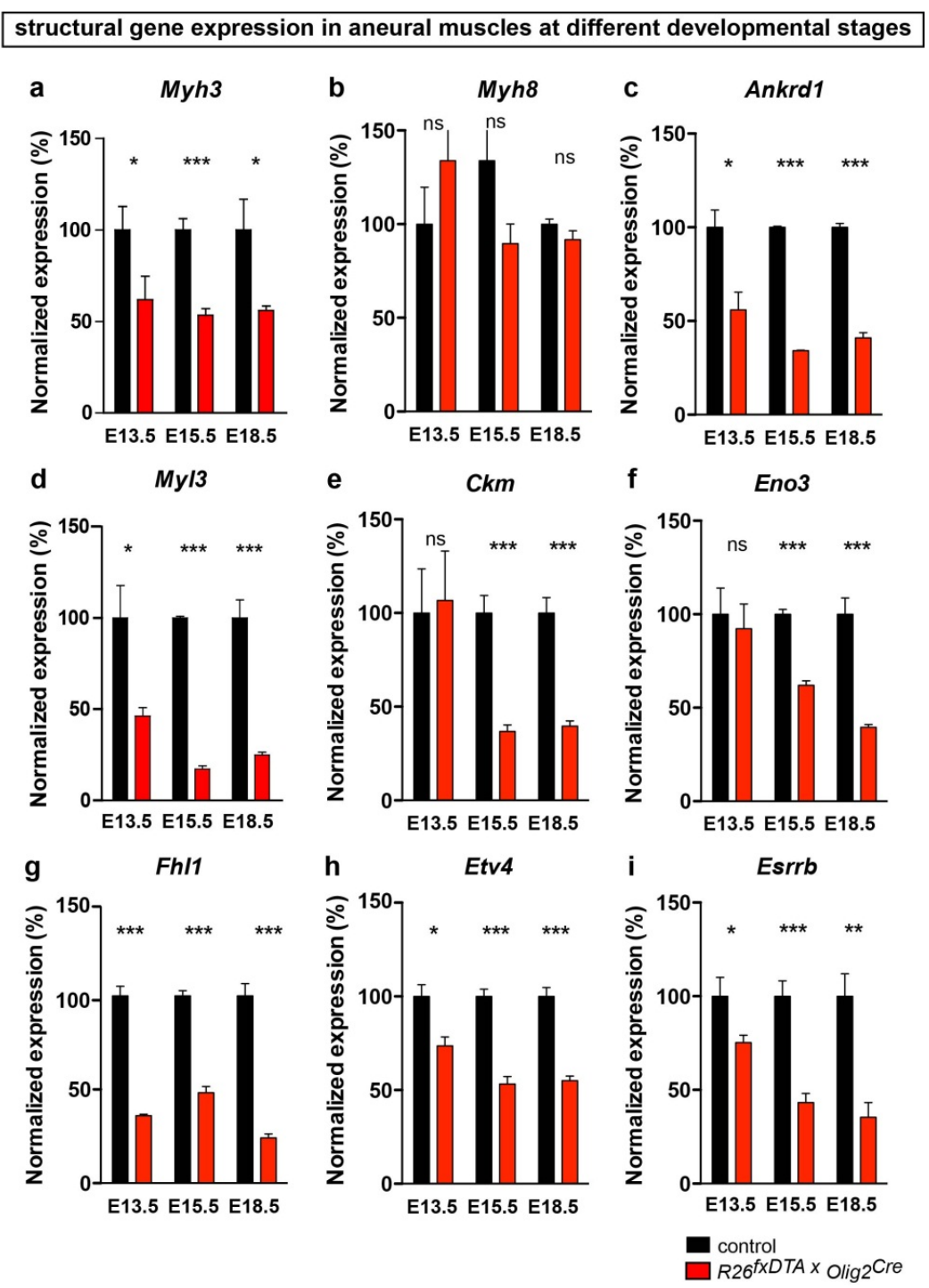

Figure 3.29: qPCR verification of structural gene expression in aneural muscles throughout different developmental stages.

(a-b) The expression of (a) embryonic isoform of myosin heavy chain (Myh3) was downregulated but the fetal isoform of myosin heavy chain (Myh8) remained unaffected. (c) Ankrd1, a hypertrophy marker, was downregulated at E13.5, indicating that bona fide hypertrophy is not the cause for increase in CSA of mutant fibers. (d) Myosin light chain (Myl3) expression was reduced drastically just a few days after myogenesis was initiated. (e-g) Hallmark of maturation of embryonic muscles into fetal muscles is represented by the expression of (e) Muscle creatine kinase ( $\mathrm{Ckm})$, (f) Forkhead LIM protein (Fhl1) and (g) beta Enolase (Eno3) genes. (h-i) Genes encoding muscle spindle markers (h) Etv4 and (i) Esrrb were also downregulated, suggesting that formation of muscle spindles might be impaired without motor neurons. (Two-tailed Student's $t$ test with unequal variance, $\min \mathrm{n}=3$ per genotype, per stage). ${ }^{* * *}$, $\mathrm{p}<0.001 ;{ }^{* *}, \mathrm{p}<0.01 ;{ }^{*}, \mathrm{p}<0.05$. 
In addition to the dependence of extrafusal myofiber formation on motor innervation, sensory innervation (proprioception) has been established to be essential for the differentiation of intrafusal myofibers associated with muscle spindles (Zelena and Soukup, 1974; Zelena, 1976), while it has recently been reported that sensory neuron axons depend on preformed motor neuron axons to successfully project to their targets (Wang et al., 2011b). Therefore, the downregulation of the gene encoding for muscle spindle marker Etv4 in aneural muscles likely reflects defective intrafusal myofiber formation and/or terminal differentiation resulting from the failure of sensory projections to limb muscle as a consequence of motor neuron removal. While no characterized function and binding ligand has yet been identified for Esrrb, an orphan nuclear receptor family, its related member Esrrg is a well-known marker of $\gamma$ motor neurons (Friese et al., 2009) (see Table 3.5 for normalized expression levels and statistical analysis).

\begin{tabular}{|c|c|c|c|c|c|c|c|c|c|}
\hline \multicolumn{10}{|c|}{ Table 3.4 Expression of candidate genes verified by qPCR } \\
\hline \multirow{2}{*}{ Gene } & \multicolumn{2}{|c|}{ E13.5 } & \multirow[b]{2}{*}{$p$-value } & \multicolumn{2}{|c|}{ E15.5 } & \multirow[b]{2}{*}{$p$-value } & \multicolumn{2}{|c|}{ E18.5 } & \multirow[b]{2}{*}{$p$-value } \\
\hline & control & mutant & & control & mutant & & control & mutant & \\
\hline Sapd & $100 \pm 11.74$ & $38.46 \pm 8.10$ & ** & $100 \pm 3.74$ & $41.95 \pm 0.79$ & *** & $100 \pm 10.16$ & $34.73 \pm 2.26$ & *** \\
\hline Myfd & $100 \pm 8.48$ & $59.13 \pm 5.52$ & * & $100 \pm 4.54$ & $54.60 \pm 4.34$ & *** & $100 \pm 11.67$ & $52.01 \pm 2.47$ & ** \\
\hline Snaid & $100 \pm 25.72$ & $26.67 \pm 5.78$ & * & $100 \pm 2.70$ & $23.02 \pm 2.24$ & *** & $100 \pm 8.98$ & $35.67 \pm 4.00$ & *** \\
\hline Fgfd & $100 \pm 18.72$ & $32.45 \pm 6.23$ & * & $100 \pm 1.57]$ & $42.05 \pm 0.56$ & 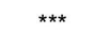 & $100 \pm 14.11$ & $57.55 \pm 4.38$ & * \\
\hline Foxdm & $100 \pm 17.27$ & $175.61 \pm 12.13$ & ns & $100 \pm 10.58$ & $68.11 \pm 6.40$ & * & $100 \pm 44.55$ & $46.26 \pm 7.95$ & ns \\
\hline Hhd & $100 \pm 20.39$ & $109 \pm 21.73$ & ns & $100 \pm 3.44$ & $70.80 \pm 3.84$ & ** & $100 \pm 6.59$ & $42.42 \pm 4.10$ & ** \\
\hline Cxcld & $100 \pm 16.72$ & $118.4 \pm 46.25$ & ns & $100 \pm 1.58$ & $41.52 \pm 2.35$ & *** & $100 \pm 10.55$ & $36.59 \pm 3.55$ & ** \\
\hline Pkctd & $100 \pm 9.11$ & $93.93 \pm 8.96$ & ns & $100 \pm 2.05$ & $67.20 \pm 4.18$ & ** & $100 \pm 10.44$ & $72.67 \pm 2.99$ & * \\
\hline
\end{tabular}

Table 3.4: List of positively identified candidate genes in aneural muscles.

Downregulated candidate genes in muscles of control and $\mathrm{R} 26^{f x D T A} x \mathrm{Olig} 2^{\mathrm{Cre}}$ mice were verified by $\mathrm{qPCR}$ at E13.5, E15.5 and E18.5. ${ }^{* *}, \mathrm{p}<0.001{ }^{* *}, \mathrm{p}<0.01 ;{ }^{*}, \mathrm{p}<0.05 ;$ ns, not significant. 


\begin{tabular}{|c|c|c|c|c|c|c|c|c|c|}
\hline \multirow{2}{*}{ Gene } & \multicolumn{2}{|c|}{ E13.5 } & \multirow[b]{2}{*}{$p$-value } & \multicolumn{2}{|c|}{ E15.5 } & \multirow[b]{2}{*}{$p$-value } & \multicolumn{2}{|c|}{ E18.5 } & \multirow[b]{2}{*}{$p$-value } \\
\hline & control & mutant & & control & mutant & & control & mutant & \\
\hline Ankrd1 & $100 \pm 9.21$ & $55.90 \pm 9.42$ & * & $100 \pm 0.66$ & $34.11 \pm 0.27$ & 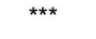 & $100 \pm 2.05$ & $41.06 \pm 2.76$ & $* \star \star *$ \\
\hline Ckm & $100 \pm 23.61$ & $106.7 \pm 26.33$ & ns & $100 \pm 9.32$ & $36.75 \pm 3.49$ & 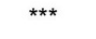 & $100 \pm 8.17$ & $39.60 \pm 2.79$ & *** \\
\hline Eno3 & $100 \pm 14.01$ & $92.25 \pm 13.24$ & ns & $100 \pm 2.57$ & $62.02 \pm 2.34$ & $\star \star \star \star$ & $100 \pm 8.75$ & $39.58 \pm 1.50$ & $\star * \star$ \\
\hline Esrrb & $100 \pm 10.04$ & $75.20 \pm 4.04$ & ns & $100 \pm 8.17$ & $43.33 \pm 4.88$ & ** & $100 \pm 12.01$ & $35.39 \pm 7.83$ & $\star *$ \\
\hline Etv4 & $100 \pm 6.22$ & $73.64 \pm 4.68$ & * & $100 \pm 3.79$ & $53.30 \pm 4.02$ & $\star * * *$ & $100 \pm 2.048$ & $41.06 \pm 2.76$ & $* * *$ \\
\hline Fhl1 & $100 \pm 5.13$ & $36.01 \pm 0.81$ & *** & $100 \pm 2.69$ & $48.30 \pm 3.38$ & $* * *$ & $100 \pm 6.49$ & $24.99 \pm 1.66$ & $* * *$ \\
\hline Myh3 & $100 \pm 12.79$ & $49.90 \pm 6.81$ & * & $100 \pm 6.23$ & $53.35 \pm 3.61$ & *** & $100 \pm 16.89$ & $56.03 \pm 2.37$ & * \\
\hline Myh8 & $100 \pm 19.71$ & $133.8 \pm 37.65$ & ns & $100 \pm 7.22$ & $89.55 \pm 10.54$ & ns & $100 \pm 2.70$ & $91.78 \pm 4.75$ & ns \\
\hline Myl3 & $100 \pm 17.65$ & $46.16 \pm 4.66$ & * & $100 \pm 0.90$ & $17.21 \pm 1.69$ & $\star * *$ & $100 \pm 9.79$ & $24.81 \pm 1.58$ & 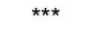 \\
\hline
\end{tabular}

Table 3.5: List of downregulated structural genes in aneural muscles.

Negatively regulated structural genes in muscles of control and R26fxDTAxOlig2Cre mice verified by $\mathrm{qPCR}$ at various developmental stages. Removing motor neurons led to significant downregulation of structural genes such as Myh3, Ankrd1, Myl3, Ckm, Essrb, Etv4, Fhl1, and Eno3. *** ${ }^{*}<0.001 ;{ }^{* *}, \mathrm{p}<0.01 ;{ }^{*}, \mathrm{p}<0.05 ; \mathrm{ns}$, not significant.

\subsubsection{Embryonic denervation utilizes a set of molecules distinct from adult denervation pathway}

The pathways underlying adult denervation are surfacing, including a set of genes upregulated following denervation induced by sciatic nerve transection in the adult is believed to be responsible for translating the lack of motor innervation into muscle atrophy (Moresi et al., 2010; Macpherson et al., 2011). To test whether there are parallels between the molecular pathways induced adult and embryonic denervation, I selectively investigated the expression of genes implicated in adult denervation after motor neuron ablation in the embryo. Notably, microarray and RNAseq data showed no significant difference in the expression level of genes upregulated after denervation in the adult (Figure 3.30 a). 


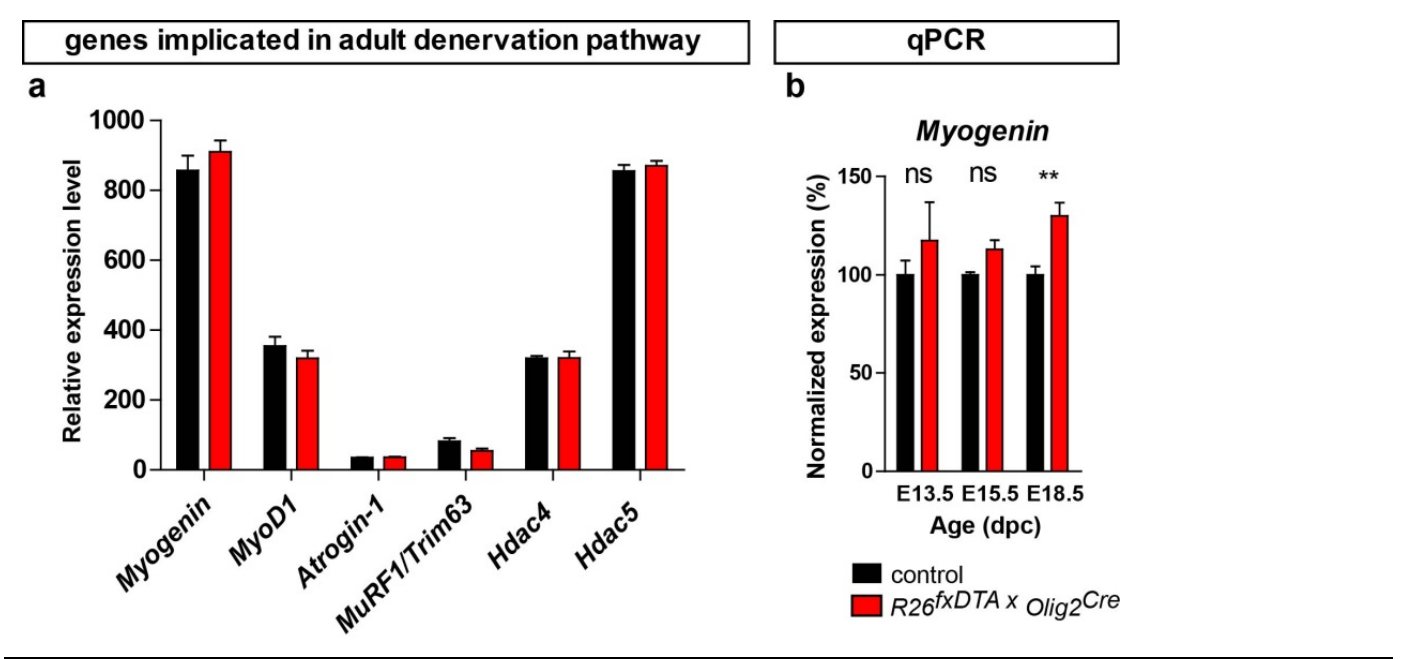

Figure 3.30: Embryonic denervation operates through a distinct pathway than adult.

(a) No significant difference in the expression level of a set of genes implicated during adult denervation was detected between E15.5 control and R26fxDTA $x$ Olig2 ${ }^{\text {Cre }}$ muscles. Transcripts of E3 ubiquitin ligases, Atrogin-1 and MuRF1/Trim63 were exceptionally low in E15.5 muscles. (b) qPCR expression of analysis of myogenin, a member of the MRF family, throughout different developmental stages. Myogenin transcripts were increased in mutant muscles at E18.5 significantly. Data are presented as normalized values to control. (Two-tailed Student's $t$ test with unequal variance, $\min \mathrm{n}=3$ per genotype, per stage). $\left.{ }^{* * *}, \mathrm{p}<0.001 ;{ }^{* *}, \mathrm{p}<0.01 ;{ }^{*}, \mathrm{p}<0.05\right)$.

Additionally, the transcripts of E3 ubiquitin ligases, which appear to be the final effectors in mediating adult muscle atrophy (Bodine et al., 2001; Moresi et al., 2010; Macpherson et al., 2011), were not differentially regulated between control and $R 26^{\text {fXDTA }} x \mathrm{Olig} 2^{\mathrm{Cre}}$ embryos, and had a subthreshold expression ( $<100$ relative units) in E15.5 embryonic hindlimb muscles. This suggests that embryonic denervation acts through pathways differing from the adult denervation pathways involving Atrogin-1 and MuRF1/Trim63 E3 ubiquitin ligases. Likewise, myogenin (a member of the MRF family), a developmental gene important for embryonic myogenesis (Eftimie et al., 1991; Borycki and Emerson, 1997) whose expression was shown to reappear after nerve transection postnatally (Moresi et al., 2010; Macpherson et al., 2011) was not differentially regulated in the aneural $R 26^{f x D T A} x \mathrm{Olig} 2^{\mathrm{Cre}}$ embryos. To determine if there was a period during which myogenin was impacted during development, I tested for 
myogenin gene expression using $\mathrm{qPCR}$ on control and aneural muscles at distinct embryonic stages. Indeed, it was confirmed that while levels of myogenin transcripts were unchanged at E13.5 and E15.5 (early embryonic stages), it was however slightly upregulated in the muscles of $\mathrm{R}^{66^{f x D T A} x \mathrm{Olig} 2^{\mathrm{Cre}}}$ mice at E18.5 (Figure $3.30 \mathrm{~b}$ ). The reason for the latter observation is unclear, but might be attributed to increased satellitederived myogenesis involving myogenin observed in late-gestation aneural muscle. Alternatively, this observation may reflect a transition to a more postnatal/adult-like stage of myogenesis with the end of gestation and thus, the partial initiation of an adult-like denervation pathway in E18.5 aneural embryos.

\subsubsection{Motor innervation regulates Ntf5 and Ntrk2 transcript levels in muscles}

Early studies have underlined the importance and expression of neurotrophic factors after motor neuron elimination (Houenou et al., 1991; Oppenheim, 1991). During development, the skeletal muscle is identified as an essential source of neurotrophic support for motor neurons (Griesbeck et al., 1995; Chevrel et al., 2006). Previous studies have also demonstrated that denervation in the adult resulted in changes in muscle neurotrophin level (Griesbeck et al., 1995; Stuerenburg and Kunze, 1998; Sakuma et al., 2001). Hence, it would be interesting to investigate motor neuronmediated regulation of neurotrophic factor expression in the muscles. Herein, I have used qPCR to assess the response of genes encoding various neurotrophic factors and their receptors to the lack of motor innervation in E15.5 muscles (Figure 3.31). Out of the 7 neurotrophic factors and receptors tested, the transcript levels of neurotrophin $4 / 5$ (Ntf5) and neurotrophic tyrosine kinase type 2 (Ntrk2) were the only ones significantly altered in muscles of $\mathrm{R}^{26^{f x D T A}} x \mathrm{Olig} 2^{\mathrm{Cre}}$ mice compared to controls (Figure $3.31 \mathrm{c}$ and d). Contrary to preceding studies that described $\mathrm{Nt} 4 / 5$ as being neurallydependent after observing that blockade of adult postsynaptic AChRs led to reduced 
Nt4/5 expression (Sakuma and Yamaguchi, 2011), Ntf5 transcripts were instead upregulated in my findings.

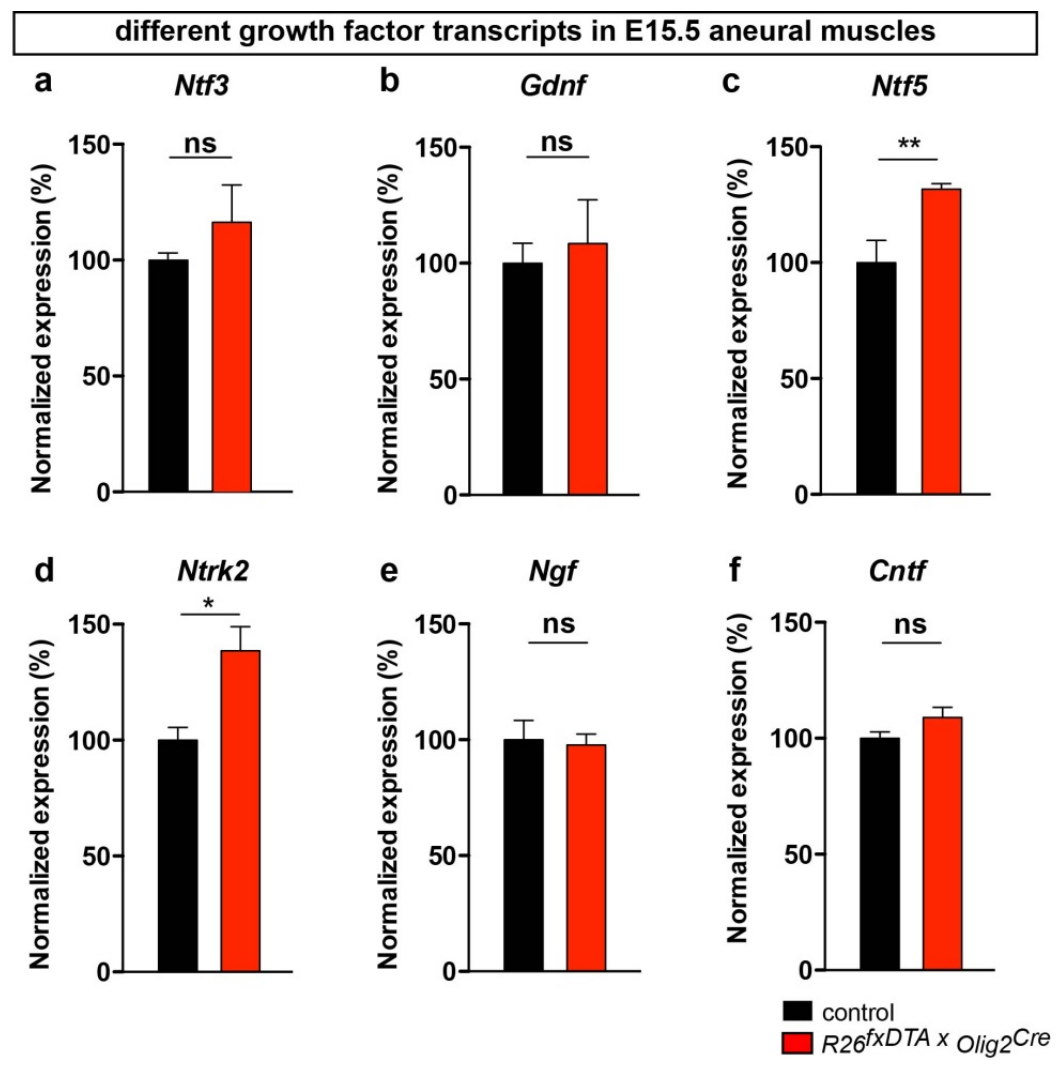

Figure 3.31: qPCR verification of growth factors and receptors expression in aneural E15.5 muscles. (a-f) No significant difference could be detected in the expression of (a) neurotrophin 3 (Ntf3), (b) glialderived nerve factor $(G d n f)$, (e) nerve growth factor $(N g f)$ and (f) ciliary nerve trophic factor (Cntf) gene in R26fxDTA xOlig2Cre muscles. Transcript levels of (c) neurotrophin $4 / 5$ (Ntf5) and (d) tyrosine kinase receptor type 2 (Ntrk2) were differentially regulated in mutant muscles by 1.31 and 1.40 fold, respectively. Data are represented as normalized values to controls. (Two-tailed Student's $t$ test with unequal variance, $\min \mathrm{n}=3$ per genotype). ${ }^{* * *}, \mathrm{p}<0.001 ;{ }^{* *}, \mathrm{p}<0.01 ;{ }^{*}, \mathrm{p}<0.05$.

This disparity may be a stage-dependent response in the requirement for motor innervation, supported by the observation that Ntrk2 which encodes for a membrane protein receptor that binds specifically to Bdnf and $\mathrm{Nt4} / 5$ was correspondingly upregulated (Figure $3.31 \mathrm{~d}$ ). Genes encoding other neurotrophic factors which were 
tested but displayed unaltered expression included neurotrophin 3 (Ntf3), glial-cellline-derived neurotrophic factor $(G d n f)$, nerve growth factor $(N g f)$ and ciliary neurotrophic factor (Cntf) (Figure 3.31 a, b, c, and f). Surprisingly, the potent neurotrophic factor Gdnf that is known to be strongly expressed during early myogenesis to promote survival of motor neurons (Sakuma and Yamaguchi, 2011) did not display differential gene expression after motor neuron ablation. Additionally, the transcripts of brain-derived neurotrophic factor $(B d n f)$, claimed to be differentially expressed in skeletal muscles during numerous pathological and physiological states in the adult (Chevrel et al., 2006) were undetected in E15.5 muscles (data not shown). This may be attributed to: (1) the low expression or absence of Bdnf in the embryonic muscles or (2) the absence of Bdnf expression in the muscles of both embryo and adult but this question remains controversial (Griesbeck et al., 1995; Sakuma et al., 2001).

\subsection{Gene expression profiles in paralyzed muscles of $\mathrm{R}_{2} 6^{f x T e N T} x \mathrm{Olig} 2^{\mathrm{Cre}}$ mice}

As described in earlier sections, blocking evoked neuromuscular transmission in R26 ${ }^{f T C N T} x O l i g 2^{\text {Cre }}$ mice only partially recreates the effects on muscle caused by wholesale removal of motor neurons or neuromuscular transmission in $R 26^{f x D T A} x$ Olig2 ${ }^{C r e}$ mice or Chat ${ }^{-/}$mice, respectively. I therefore tested to what extent the identified core set of genes de-regulated upon motor neuron removal would be affected upon blocking evoked neuromuscular transmission. Hence, transcriptome profiling was performed using hindlimb muscles of E15.5 R2 $6^{f x T e N T} x O \operatorname{Oig} 2^{\text {Cre }}$ mice. Shown in Figure 3.32 is the heatmap representation of the microarray data attained (Figure $3.32 \mathrm{a}-\mathrm{b}$ ). Altogether, only 25 genes were detected as significantly downregulated and 9 genes were upregulated in the paralyzed muscles of $R 26^{f x T e N T} x$ Olig2 ${ }^{\text {Cre }}$ mice (Figure $3.32 \mathrm{c}-\mathrm{d}$ ). The markedly smaller group of genes detected as being differentially regulated likely reflects the lower degree of muscle loss and myofiber terminal differentiation observed in $R 26^{f x T e N T} x O \operatorname{lig} 2^{C r e}$ as compared to 
R26 ${ }^{f x D T A} x$ Olig2 ${ }^{\text {Cre }}$ mutants. The question to what extent the molecular pathways regulated by neuromuscular innervation would overlap with those regulated by neuromuscular transmission thus awaits the transcriptome analysis of muscle gene expression in Chat-deficient mice lacking both, evoked and spontaneous ACh release at the neuromuscular junction.
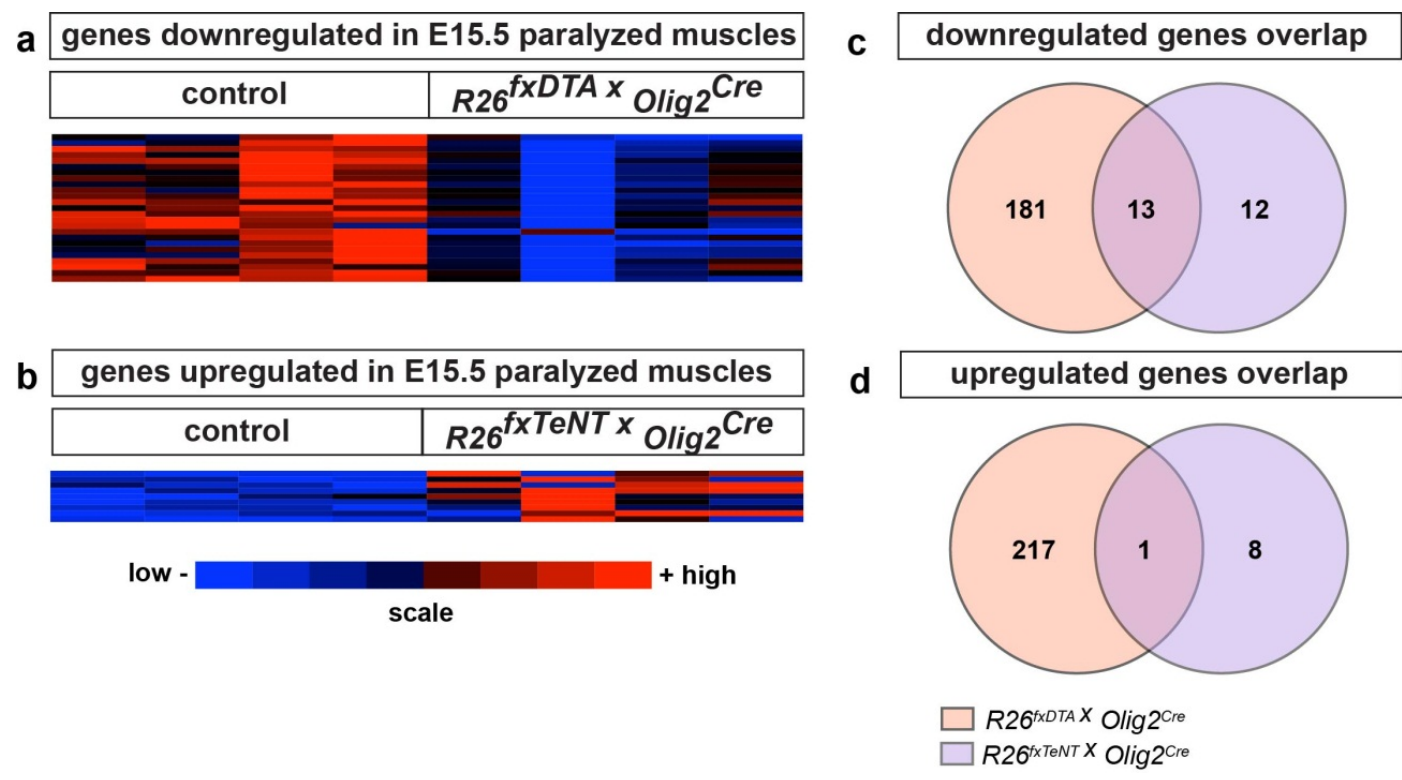

\footnotetext{
Figure 3.32: Gene profile of E15.5 R26 ${ }^{f x T e N T} x O l i g 2^{C r e}$ muscles.

(a-b) Heatmap representation of differentially regulated genes in $R 26^{f x T e N T} x O \operatorname{Oig} 2^{\text {Cre }}$ muscles. (a) 25 negatively regulated and 9 positively regulated genes were identified in E15.5 muscles of mice after blocking evoked neurotransmission. Red, high level of expression; Blue, low level of expression. (c-d) Venn diagrams comparing overlap of differentially (c) downregulated and (d) upregulated genes in $R 26^{f x D T A} x O \operatorname{lig} 2^{\mathrm{Cre}}$ and $\mathrm{R} 26^{\mathrm{fxTeNT}} \mathrm{OOlig} 2^{\mathrm{Cre}}$ muscles. Genes were sorted accordingly to the following criteria: $\geq 1.5$ fold-change and $p$-value $\leq 0.05$. The threshold level for gene expression was set to $\geq 100$ relative units.

Nevertheless, the observation that two genes encoding myogenic factor and sarcomere-associated protein Sapd and Myfd which were affected both by motor neuron ablation as well as evoked neuromuscular transmission blockade implicates both factors as prime candidates for coordinating the response of myoblasts towards innervation.
} 


\section{Discussion}

\subsection{Addressing the role of innervation in embryonic myogenesis}

Previous studies regarding the role of innervation on myogenesis gave rise to two divergent conclusions: (1) first, primary myotube formation is dependent on innervation, while secondary myotube formation is indirectly affected by denervation due to their reliance on the presence of primary myotubes (Ontell et al., 1988; Condon et al., 1990; Fredette and Landmesser, 1991b), and (2) second, primary myotubes form cell autonomously whereas the formation of secondary myotubes requires innervation (Butler et al., 1982; McLennan, 1983b; Ross et al., 1987; Wilson and Harris, 1993). These discrepancies likely arose due to the variability in the methodology available at the time these studies were conducted, namely the mechanical surgical ablation of embryonic spinal cord or the whole-embryo application of neural and/or muscle activity blockers to address the role of innervation in embryonic myogenensis. Methodologically, inducing denervation by neural tube removal or partial spinal cord transection is inherently non-specific, since other cell types in the spinal cord (i.e., interneurons, glial cells) besides motor neurons are also eliminated. Additionally, with this approach there is no control over the completeness of denervation or possible reinnervation, as well as the side effects of surgery on the morphogenesis of embryos. Further, application of neural and/or muscle activity blockers (for e.g., d-tbc, curare, $\alpha$ BTX, TTX) were largely inconclusive because application of paralyzing agents at early stages could affect the pattern of activation of immature muscles, leading to artifacts such as tension-induced damage (Landmesser and Szente, 1986). In addition, discrepancies in results may have arisen from the incomplete elimination of neural influence resulting from the incomplete blockade of postsynaptic receptors or neurotransmission. For instance, drugs meant to perturb neurotransmitter release or neurotransmission may do so by inhibiting evoked but not spontaneous release (Harris, 1981; Duxson, 1982; Houenou et al., 1990; Dahm and Landmesser, 1991; 
Deitcher et al., 1998). Because of this, developmental programs that were concluded to be activity-independent may actually require spontaneous activity that was not eliminated by a particular drug (Misgeld et al., 2002). Moreover, drugs used in previous studies may have nonspecific detrimental effects on myogenesis, such as the commonly used $\beta$-BTX to destroy nerve, which was reported to be directly myotoxic (Creazzo and Sohal, 1981). Lastly, differences in the time of induced denervation, neurotransmission blockade or paralysis could give rise to markedly varying outcomes. For instance, due to technical limitations, the earliest time point at which spinal cord ablation was possible in mouse embryos was at E14.5 (Hughes and Ontell, 1992) an inappropriate stage to study the impacts of innervation on myogenesis because the motor nerve has already projected into the muscles and primary myogenesis has ended. Thus, impacts of neurotransmission blockade on myogenesis generally correlated with the developmental stage at which neurotransmission blockers were administered (McLennan, 1983b; Sohal and Sickles, 1986; Fredette and Landmesser, 1991a). Taken together, due to these method-inherent variations the exact role of innervation during embryonic myogenesis remains unclear.

To circumvent ambiguities inherent to previous approaches and to precisely address the role of innervation in myogenesis, I have employed a molecular genetic method in mouse that allows for the wholesale removal of motor neurons from the outset, thus excluding any neural influence on muscle development. In addition, through this method, detrimental consequences on embryo morphogenesis after surgical manipulation, factors arising from stage differences due to delayed timing of denervation as well as incomplete or nonspecific drug-effects can be circumvented. As a proof of principle, in both $226^{f x D T A} x O \operatorname{Oig} 2^{\text {Cre }}$ and Isl2 ${ }^{f x D T A} x O \operatorname{Oig} 2^{C r e}$ mice, only motor neurons and oligodendrocytes (only in $\mathrm{R}^{66^{f x D T A} x \mathrm{Olig} 2^{\mathrm{Cre}}}$ mice) were ablated whilst other cells in the spinal cord were left intact, thereby demonstrating the specificity of both mouse lines. More importantly, a similar pattern of skeletal muscle hypoplasia could be produced in both $\mathrm{R}_{2} 6^{\mathrm{fxDTA}} x \mathrm{Olig} 2^{\mathrm{Cre}}$ and Isl2 ${ }^{\mathrm{fxDTA}} \mathrm{xOlig} 2^{\mathrm{Cre}}$ mice, in which motor 
neurons are ablated at the progenitor-stage and postmitotic-stage, respectively, indicating that impacts on muscles observed were specifically caused by the lack of motor innervation. Thus, this genetic approach allowed me to selectively study the impact of innervation on embryonic myogenesis while eliminating variables inherent to mechanical or laser ablation of the spinal cord, and pharmacological application to create aneural or paralyzed muscles.

\subsection{Primary and secondary myotubes form aneurally}

That functional innervation is required for maintaining muscle integrity and/or survival is widely accepted (McLennan, 1994), but to what extent muscle development, specifically primary or secondary myogenesis or myotube maintenance are vulnerable to denervation remains a contested issue. Contrary to the two opposing views on the dependence of either primary or secondary myogenesis on innervation, my results obtained from selective and complete genetic suppression of innervation show that denervation affects both primary and secondary myotubes. I found that primary myotubes form in muscles of $R 26^{f x D T A} x O \operatorname{Oig} 2^{\mathrm{Cre}}$ mice (evidenced by the presence of slow $\mathrm{MyHC}^{+}$fibers), but eventually degenerate via apoptosis by the end of gestation. This was based on my observation that reduced muscle mass was manifested in aneural embryos at stages prior to initiation of secondary myogenesis. Thus, the initial generation of primary myotubes does not appear to depend presence of nerves. Rather, innervation seems to be crucial for myofiber terminal differentiation (discussed further below).

Previous studies based on $\beta$-BTX injection in rodents concluded that the generation of secondary, but not primary myotubes is a nerve-dependent process (Ross et al., 1987; Wilson and Harris, 1993). Contrastingly, I found that muscle fibers with EdU+ central nuclei, which are generated during secondary myogenesis, were present in motor neuron-deficient $\mathrm{R} 26^{f \times D T A} x \mathrm{Olig} 2^{\mathrm{Cre}}$ mice. I further found that in these mice, absolute 
muscle mass increases as development proceeds even after the period of primary myogenesis; the source of which could only come from the continuous addition of newly formed secondary fibers, until the appearance of satellite cells at E16.5. The disparity between my results and that of earlier studies (Ross et al., 1987; Wilson and Harris, 1993) most likely come from the latter's use of $\beta$-BTX to destroy nerves and its inherent problems (see above). However, relative muscle mass was progressively reduced even after primary myogenesis in $\mathrm{R}_{2} 6^{f \times D T A} x \mathrm{Olig} 2^{\mathrm{Cre}}$ mice when normalized to controls, and is likely attributed to the degeneration of secondary myotubes. Hence, my results conclusively show that in genetically-induced aneural muscle, secondary myofibers are generated, but eventually degenerate in the continued absence of the nerve. However, due to current technical limitations, the extent (if at all) of compromised secondary myotube generation in these embryos, which could likely be a consequence of ongoing primary myofiber degeneration and thus lack of structural support (Kelly and Zacks, 1969) for their formation, can currently not be ruled out.

Fredette and Landmesser's (1991) estimate of the ratio of primary to secondary myotubes was derived from chick muscle sections stained with an anti-slow myosin (avian slow MyHC 2 and 3 isoforms) antibody. However, because the amount of slow MyHC 2 and 3 isoforms was reduced in denervated (and paralyzed) chick myotubes (Crow and Stockdale, 1986; McLennan, 1994), the antibody may not sufficiently detect affected myotubes, and may thus give an inaccurate representation of the proportion of primary to secondary myotubes. In my studies, I have also used an antibody recognizing slow myosin isoform to identify primary myotubes, but this particular myosin isoform (Myh7) was unaffected after denervation in $\mathrm{R}_{2} 6^{f x D T A} x \mathrm{Olig} 2^{\mathrm{Cre}}$ mice. Another study supporting the hypothesis by Fredette and Landmesser (1991) was based on inducing denervation in E14.5 mouse embryos via laser ablation of the lumbosacral spinal cord (Hughes and Ontell, 1992). A major caveat in the latter experiment is the differentiation of primary and secondary myotubes based on size. Although primary myotubes are larger in size when compared to secondary myotubes 
at the onset secondary myogenesis, secondary myotubes also rapidly increase in diameter as development proceeds (McLennan, 1994). Therefore, while this technique is informative at early stages of embryogenesis, it is less so at later stages when primary and secondary myotubes have comparable sizes and may be indistinguishable from one another, thus preventing a precise estimation of primary to secondary fiber ratios. Interestingly, to my knowledge, none of the previous studies addressed possible impacts denervation or lack of neuromuscular transmission may have on the subsequent differentiation of myotubes. Although muscles were formed aneurally in $R 26^{f x D T A} x \mathrm{Olig} 2^{\mathrm{Cre}}$ mice, I observed that they indeed exhibited profound morphological defects such as hypertrophy and terminal differentiation blockade that preceded myotube degeneration. These events were further heralded by impairments in the regulation of the expression of several genes encoding essential components of the skeletal muscle fiber contractile apparatus, as demonstrated by the early downregulation of these genes at E13.5 prior to detection of muscle loss. Thus, while primary and secondary myogenesis are initiated in the absence of innervation, they still depend on innervation for eventual survival.

\subsection{Paradoxical hypertrophy of aneural muscle fibers}

It is established that in the adult after denervation, muscle fibers undergo atrophy brought about by increased protein breakdown (Bodine et al., 2001; Moresi et al., 2010). Unexpectedly, although skeletal muscle hypoplasia and decreased fiber density was observed in $226^{f x D T A} x \mathrm{Olig}_{2} \mathrm{Cre}$ mice following motor neuron removal, the individual muscle fibers exhibited an increase in the average CSA (cross sectional area), suggesting that the muscle fibers hypertrophied in the absence of motor innervation. Hypertrophy is a multifaceted process that can be activated through different mechanisms. However, the larger diameters of aneural fibers encountered were probably not caused by classical muscle hypertrophy, because genes encoding 
proteins involved in the underlying pathways, including Insulin-like growth factor 1Akt kinase/Protein kinase B, (Igf-Akt1/Pkb) and their downstream effectors, such as mammalian target of rapamycin (Mtor), S6 kinase (S6k) and Glycogen synthase kinase-3 beta (Gsk3b) (Schiaffino and Mammucari, 2011), were not differentially regulated by embryonic dennervation according to transcriptome data that I obtained in this study. Additionally, a downregulation in the expression of genes encoding putative hypertrophic markers, Ankrd1 and Ankrd2, which are involved in dilated cardiomyopathy (DCM) and skeletal muscle hypertrophy in response to prolonged mechanical stretch (Kojic et al., 2004; Moulik et al., 2009), respectively, was observed in aneural $\mathrm{R}_{2} 6^{\mathrm{fxDTA}} \mathrm{XOlig} 2{ }^{\mathrm{C} r e}$ muscles. Thus, the mutant muscle fibers with a larger CSA may undergo compensatory hypertrophy as a consequence of their degeneration and/or defective separation of secondary from primary fibers.

Consistently, it was previously found that the slow anterior lattisimus dorsi (ALD) muscles in the chick (increased by $30 \%$ after 3 weeks of denervation), while other reports suggested that in adult rat and mouse denervation induces an increase in fiber area and size (Jung and Wu, 1962; Feng and Lu, 1965; James, 1976; Connold et al., 1993). Notably, the degenerative changes observed in $\mathrm{R}_{2} 6^{f \times D T A} x \mathrm{Olig} 2^{\mathrm{Cre}}$ muscle fibers, such as elevated apoptosis and centralized myonuclei, were akin to those described in denervated atrophic muscles (Jung and $\mathrm{Wu}, 1962$ ). A clue might come from genes, including Kcnc4 and Scn3b encoding skeletal muscle-specific ion channels that were also negatively affected by the lack of motor neurons. It is therefore conceivable that the increase in muscle fiber CSA in these embryos was an additive degenerative effect caused by the abnormal influx and efflux of ions into and out of the muscle cell. Denervated embryonic "swollen" muscle fibers have also been described by Wilson and Harris (1993), which was ascribed to degeneration. Alternatively, since secondary fibers form on the scaffold of larger primary fibers and separate from the parent fiber after maturation to be encased in its own basal lamina, it is possible that the increase in muscle fiber CSA reflects a deficiency in the separation of secondary fibers from the 
primary fibers.

Regarding potential compensatory responses to denervation, increases in $\mathrm{Pax}^{+}$ satellite cells after denervation have been reported by studies performed in adult rodents (McGeachie, 1989; Viguie et al., 1997). The number of satellite cells was highest in adult limb muscles 1 week postdenervation when compared to the contralateral innervated limb, and this proliferation sustained for about 4 weeks before the pool of satellite cell precursors finally became depleted (McGeachie, 1989; Viguie et al., 1997). Likewise, an elevation of Pax $7^{+}$cells in muscles of embryonic R26 ${ }^{f x D T A} x O \operatorname{lig} 2$ Cre mice was detected, suggesting increased proliferation and/or activation of satellite cells as a compensatory response to the progressive loss of muscle mass following denervation. Therefore, it appears that the recruitment of satellite cells after denervation is a common response both in the embryo and adult.

\subsection{Innervation drives terminal differentiation of muscle fibers}

The failure of sarcomeres to form in $\mathrm{R}_{2} 6^{f x D T A} x \mathrm{Olig} 2^{\mathrm{Cre}}$ mice indicates a failure of myotube terminal differentiation in $\mathrm{R}^{2} 6^{f x D T A} x \mathrm{Olig} 2^{\mathrm{Cre}}$ embryos. During muscle fiber terminal differentiation nascent elongated myotubes form the contractile apparatus through myofibrillogenesis that normally commences after myoblast fusion is initiated (Eftimie et al., 1991). In the adult, long-term denervation can result in a similar poorly developed contractile system in newly formed myotubes and defective terminal differentiation (Borisov et al., 2005). Research performed in chronically denervated rats by Borisov and colleagues (2005) showed that most of the underdeveloped satellite cell-derived myotubes undergo degeneration, and those remaining that had previously formed on the surface of parent myotubes beneath the basal lamina did not contain developed myofibrils. In agreement with their results, denervation during embryogenesis in $\mathrm{R}^{26^{f x D T A}} x \mathrm{Olig}_{2} \mathrm{Cre}$ mice also resulted in immature primary and 
secondary muscle fibers which contained no sarcomeres and were susceptible to apoptosis. The stage at which mice were denervated could be a possible explanation as to why Hughes and Ontell (1992) reported normal muscle fiber morphology with no terminal differentiation blockade upon denervation. Mice used in their study were denervated by laser ablation of the lumbosacral spinal cord at E14.5, a time when hindlimb muscles are already functionally innervated. Thus, existing neural influence present during E14.5 might be sufficient to drive terminal differentiation, thereby alleviating potential denervation-induced skeletal muscle fiber defects in these embryos.

Terminal differentiation, besides sarcomere formation events, also features the transcriptional activation of a battery of muscle-specific genes leading to the expression of metabolic enzymes, ion channels and neurotransmitter receptors (Eftimie et al., 1991). The switch from embryonic-type to adult-type nAChRs, a feature of muscle maturation, is similarly thought to be under the influence of innervation (Adams et al., 1995). Denervated adult muscle fibers exhibit hypersensitivity to ACh (Axelsson and Thesleff, 1959), caused by increases in levels of embryonic-type nAChR RNAs within 24-48 h postdenervation (Goldman et al., 1988). Contrary to these findings, transcripts of $\beta$-subunit (Chrnb1) of $\mathrm{nAChRs}$ were found to be 1.24-fold higher in innervated embryonic muscles compared to $226^{f x D T A} x \mathrm{Olig} 2^{\mathrm{Cre}}$ muscles (data not shown), whereas no significant differences were detected for transcripts of $\alpha-, \delta-$, $\gamma$ - and $\varepsilon$-subunits. The reason for this discrepancy is unclear, but could be due to age difference as studies performed on denervation-induced muscle-specific gene transcriptional control were mostly on adult mice. Alternatively, the initialization of muscle-AChR clusters is documented to occur around E13.5-14.5, and E15.5 (a time when transcriptome profiling was done) may be too early to observe any possible adverse effects of denervation on gene expression of AChR subunits, which may only become apparent at later stages of development. Hence, from current results it is not yet possible to conclude if motor innervation affected other AChR subunits or not, 
since their gene expression has not yet been examined beyond E15.5 in R26 fxDTA $\mathrm{Olig} 2^{\mathrm{Cre}}$ mice.

Myogenin and MyoD proteins are known to be under the influence of electrical activity, as well as to bind to enhancers or promoters of muscle-specific genes such as $\mathrm{Ckm}$ and $\mathrm{Myl}$ in order to activate their transcription during muscle differentiation (Edmondson and Olson, 1989; Lassar et al., 1989; Brennan and Olson, 1990). Consistent with this, without motor neurons, genes encoding structural proteins and enzymes such as Myl2, Myl3 and Ckm were downregulated at E13.5 or E15.5 in aneural $R 26^{f x D T A} x$ Olig2 ${ }^{\text {Cre }}$ muscles. Intriguingly, at E15.5, genes encoding both MRF proteins were not differentially expressed between control and aneural R26fxDTA $x O \operatorname{lig} 2{ }^{C r e}$ muscles, suggesting that the downregulation of muscle-specific genes was unlikely the result of modulation by myogenin and MyoD. Rather, this could normally be mediated by another closely related MRF member, Myfd, whose transcripts were congruently decreased before E15.5 in my study and which can bind to the same cis elements as myogenin and MyoD (Murre et al., 1989). In line with this hypothesis, all variants of Myfd null mice express significantly lower levels of MyHC-emb isoform (Braun and Arnold, 1995; Patapoutian et al., 1995; Zhang et al., 1995; Olson et al., 1996), indicating that Myfd can indeed regulate genes independent of other MRF proteins. The lower levels of MyHC-emb in Myfd null mice can also be reasoned to be effects from the reduction of Myf5 expression that consequently accompanies the deletion of the Myfd allele (Braun and Arnold, 1995). However, MyHC-emb transcripts were also detected at significantly lower levels in aneural $R 26^{f x D T A} x O \operatorname{Oig} 2^{\mathrm{Cre}}$ muscles with dampened Myfd gene expression, while Myf5 gene expression was unchanged. In the attempt to identify possible genes transactivated by Myfd, further analysis involving Myfd binding sites contained within enhancers of other muscle-specific genes and 'late response' genes would be helpful.

While the striated pattern characteristic of skeletal muscles was abolished from 
myotubes of $R 26^{f x D T A} x O \operatorname{Oig} 2^{C r e}$ mice, it was well preserved in myotubes of $R 26^{f T e N T} x O l i g 2^{C r e}$ mice. Nerve activity-induced effects, specifically $\mathrm{Ca}^{2+}$ transients, could be an underlying cause for this disparity observed. $\mathrm{Ca}^{2+}$ transients induced after depolarization of end plate potentials stimulated by $\mathrm{ACh}$ release from the presynaptic motor nerve and the resultant contractile activity is known to govern most aspects of myofibril assembly, protein turnover, and myofibril maintenance (Sharp et al., 1993; Berchtold et al., 2000). Specifically, spontaneous $\mathrm{Ca}^{2+}$ transients normally occur during embryonic myogenesis to phosphorylate myosin light chain kinase (Mylk) that is permissive for myosin thick filament assembly (Flucher and Andrews, 1993; Ferrari et al., 1996; Ferrari et al., 1998). These $\mathrm{Ca}^{2+}$ transients are expected to be abolished in completely aneural $R 26^{f x D T A} x \mathrm{Olig} 2^{\mathrm{Cre}}$ mice with motor neuron removal, which may, in addition to transcriptional regulation by MRFs, explain the downregulation of genes encoding several myosin heavy and light chain isoforms observed in these mutant mice. Consequently, blocking $\mathrm{Ca}^{2+}$ transients will also lead to the disruption of actin, titin and capZ organization in the sarcomere (Li et al., 2004).

The interaction between actin and myosin is required for later stages of myofibrillogenesis in the advanced consolidation of the myofibril (Flucher and Andrews, 1993). This interaction is disrupted in the absence of $\mathrm{Ca}^{2+}$ transients in $R 26^{f x D T A} x O \operatorname{lig} 2^{C r e}$ mice, which may in part underlie the loss of sarcomeric-associated gene expression in these mutant mice. Thus, assuming that only evoked neurotransmission was completely suppressed in $R 26^{f x T e N T} x O \operatorname{Oig} 2^{C r e}$ mice, spontaneous $\mathrm{Ca}^{2+}$ transients would still be generated, and might be sufficient to establish the periodic organization of sarcomeres observed in these mice. Furthermore, if terminal differentiation blockade was indeed manifested by the downregulation of Myfd, which is downregulated in both $R 26^{f x T e N T} x O l i g 2^{C r e}$ and $R 26^{f x D T A} x O l i g 2^{\text {Cre }}$ mice, Myfd does not seem to act solely to promote terminal differentiation. Rather, it may involve several other cofactors which are obligatory in regulating the differentiative properties of nascent myotubes, but which were not differentially regulated in paralyzed 


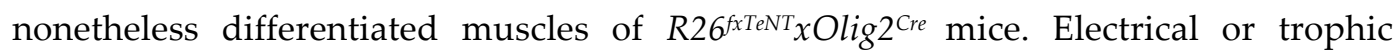
activity derived from the motor nerve has been suggested to have significant effects on the terminal differentiation of myotubes (Borisov et al., 2005). Herein, $\mathrm{Ca}^{2+}$ transients can be regarded as one of many outcomes of neural influence on myogenesis, triggering downstream pathways that support terminal differentiation of myotubes. As $\mathrm{Ca}^{2+}$ transients can also arise from cholinergic-driven spontaneous activity, it is pertinent to thoroughly examine the sarcomere structure and organization, in addition to gene expression, in muscles of motor neuron Chat-deficient mice in order to uncover possible roles for ACh-mediated spontaneous activity in myogenesis.

\subsection{Primary and secondary myotubes are formed during embryonic paralysis}

Removal of neurotransmission in $\mathrm{R}^{2} 6^{\mathrm{fx} T \mathrm{~N} T} x \mathrm{Olig} 2^{\mathrm{Cre}}$ mice resulted in markedly milder skeletal muscle hypoplasia and a later onset of muscle loss when compared to mice with wholesale motor neuron ablation. Intriguingly, absence of neuromuscular transmission in Chat null mice appear to completely mirror the effects on muscle observed in aneural embryos. The later loss in muscle mass in older $226^{f x T e N T} x O \operatorname{Olig} 2^{\mathrm{Cre}}$ embryos strongly suggest subsequent degradation of later-generated secondary fibers, although it cannot be ruled out that paralyzed primary myotubes may also have a delayed degeneration. It is noteworthy that TeTx (tetanus toxin) has been shown to act differentially in blocking spontaneous and evoked neurotransmission. TeTx completely blocks evoked (action potential-dependent) neurotransmission whereas it only reduces spontaneous quantal release by preventing the fusion of docked synaptic vesicles with the plasma membrane of nerve endings (Humeau et al., 2000). In the crayfish motor synapses, TeNT (TeTx light chain) does not affect the frequency of spontaneous events whereas these were reduced moderately in transgenic TeNT-

expressing Drosophila larval neuromuscular synapses (Sweeney et al., 1995). This difference in the response of spontaneous and evoke exocytosis to TeNT has been 
proposed to be in part attributed to the possible different apparatus underlying their vesicle release (Broadie et al., 1995; Deitcher et al., 1998; Yoshihara et al., 1999). Assuming that spontaneous release is still present even at a reduced rate in the $R 26^{f x T e N T} x$ Olig2 ${ }^{C r e}$ mice, it can be hypothesized that constant spontaneous release is likely to be critical for early myogenesis, before neuromuscular apparatus are properly specialized and before evoked action potentials are initiated. This hypothesis can thereby explain the milder skeletal muscle phenotype observed in TeNT mutants in

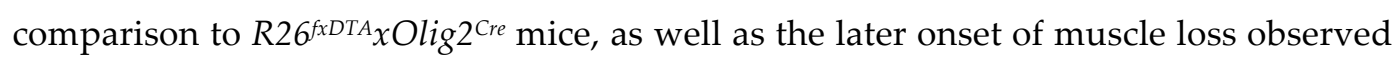
during advanced stages of development (when nerve-evoked activity becomes predominant). Alternatively, the reason why skeletal hypoplasia was less severe in $R 26^{f x T e N T} x$ Olig2 ${ }^{\text {Cre }}$ mice could be because TeNT only cleaves synaptobrevin which is potent in preventing small vesicle fusion (neurotransmitter-containing vesicles), but not dense core vesicles containing peptides. If the exocytosis of dense core vesicles is unaffected by TeNT, contents in dense core vesicles would still be periodically delivered to developing muscles and might be supportive of the formation, differentiation and/or maintenance of myotubes. However, while it is expected that vesicle release is blocked in $R 26^{f x T e N T} x O \operatorname{Oig} 2^{\text {Cre }}$ mice, it remains to be directly tested whether or not spontaneous vesicle release would still be present in the neuromuscular junctions of $R 26^{f x T e N T} x O \operatorname{lig} 2^{C r e}$ embryos or whether these results were due to insufficient TeNT expression levels resulting in an incomplete block of neuromuscular transmission.

Previous work using paralyzing agents (d-tbc, $\alpha$-BTX) have pointed out that the nerve supports secondary myotube formation (Sohal and Holt, 1980; Fredette and Landmesser, 1991b; Ashby et al., 1993a) and hinted towards ACh as having a trophic effect on myogenesis (Drachman, 1967). Even so, the extent of the requirements for ACh during embryonic myogenesis should not be conclusively determined solely by observing the effects caused by preventing the activation of postsynaptic acetylcholine receptors alone, because effects observed may have come from nonspecific activity of 
drugs administered (see above). Besides $R 26^{f x T e N T} x O \operatorname{Oig} 2^{\text {Cre }}$ mice, this study also used Chat null mice with both spontaneous and evoked ACh neurotransmission blockade (Brandon et al., 2003). Mice lacking Chat were also born with complete paralysis (Brandon et al., 2003), and phenocopied skeletal muscle defects in R26fxDTA $x$ Olig2 $\mathrm{Cre}$ mice. This finding is in line with the hypothesis that spontaneous activity is important in early stages of muscle development whereas evoked activity plays an indispensable role during later stages of development. Moreover, the phenotypic stunted muscle mass in Chat null mice is likely to be attributed to the loss of ACh, although possibilities such as Chat having other nonenzymatic activities and nonspecific effects from Schwann cells (which have been known to express Chat in culture) cannot be ruled out (Brockes, 1984).

Despite these, the use of Chat-deficient mice permits the exclusion of effects from other trophic factors on skeletal muscle development; combining data obtained from $R 26^{f x T e N T} x O l i g 2^{C r e}$ and Chat null mice strongly suggests quantal release of $\mathrm{ACh}$ to be the main nerve-derived component responsible for the upkeep of myotubes during early stages of myogenesis (probably E12.5-E15.5) whereas action potential-dependent evoked release of ACh is supportive of myotube survival after E15.5 when there is increasing evoked activity (Moody and Bosma, 2005). This proposition substantiated with existing results, pending on the efficacy of TeNT, though attractive, does not yet address if (1) primary or secondary myotubes are preferentially affected by nerve activity and if (2) terminal differentiation of myotubes is dependent on ACh-mediated neural activity. Also, because a systemic knockout of Chat was used in this study, unlikely nonspecific effects from the lack of ACh in the sympathetic nervous system cannot be ruled out as yet. Herein, the abovementioned points can be easily addressed by the use of transgenic mice with Chat conditionally removed from motor neurons, and labeling myotubes at different stages with EdU as well as examining skeletal muscle fibers for terminal differentiation defects, which would give more conclusive results. 


\subsection{Different requirements of innervation for embryonic and postnatal muscles}

The selective genetic extirpation of motor neurons in both $R 26^{6 x D T A} x O \operatorname{Oig} 2^{\mathrm{Cre}}$ and Isl2 ${ }^{f x D T A} x \mathrm{Olig} 2^{\mathrm{Cre}}$ mice resulted in skeletal muscle hypoplasia in parallel with increased cell death mediated largely by apoptosis, demonstrating that motor innervation is required as an obligatory stimulus for the formation and continued maintenance of embryonic muscle fibers. This is evidenced by the block in terminal differentiation of aneural myotubes and progressive muscle loss in both mutant embryos.

In the adult, denervation-induced degeneration is a biphasic process that can be separated into an early phase and a late phase (Borisov et al., 2001; O'Leary et al., 2012). The early myogenic response represents a systemic reaction of the muscle to the loss of neural input, is characterized by the reactivation of myogenesis in the muscle, starting between days 10-21 postdenervation and dominates during the first 2 months following nerve transection (Borisov et al., 2001). On the other hand, during the later phase, muscle fibers gradually decrease in numbers with progressive postdenervation atrophy, with cell death accompanying the atrophic process (Borisov et al., 2001). Denervation-induced atrophy involves protein degradation that is mediated by a series of intracellular signaling networks, including the lysosomal and $\mathrm{Ca}^{2+}$-dependent proteases, ubiquitin-proteasome-mediated proteolysis, matrix metalloproteinases and autophagy (Ventadour and Attaix, 2006; Plant et al., 2009). Apoptotic pathways are also activated during adult denervation (O'Leary et al., 2012), as it has been found that inactivating caspase 3 in mice partially protects against denervation-induced skeletal muscle atrophy (Plant et al., 2009). Similarly, double knockout Bax/Bak (proapoptotic factors) mice also display $40 \%$ attenuation in muscle atrophy following denervation (O'Leary et al., 2012). However, apoptosis in denervation-induced signaling is only one of the causes of loss of muscle mass, and further cannot account for the predominant muscle fiber atrophy occuring under these conditions (Plant et al., 2009). Rather, the bulk of muscle loss is thought to be regulated by autophagy, whereas 
atrophy appears to be mediated predominantly by ubiquitin-proteasome-mediated proteolysis (Beehler et al., 2006).

Apoptosis-mediated muscle mass reduction is observed around 2 weeks after nerve transection in adult mice (O'Leary et al., 2012). In this regard, the time course for the activation of apoptotic signaling pathway resulting in muscle mass reduction ostensibly differs between embryonic and adult denervation. While caspase 3activation was detected in 2-week-denervated adult muscles, a significant elevation in cleaved caspase $3^{+}$cells could already be observed within aneural muscles of R26 ${ }^{f x D T A} x O \operatorname{Oig} 2^{\text {Cre }}$ and Isl2 ${ }^{f x D T A} x \mathrm{Olig} 2^{\mathrm{Cre}}$ mice by E13.5, approx. 3-4 days after motor neurons were ablated and 1-2 days after the initiation of primary myogenesis. This difference between the activation of denervation-induced apoptotic signaling pathway may hint at fundamental distinctions in the requirements of innervation between embryonic and postnatal muscles. Consistent with this, biochemical and structural muscle properties that were altered after embryonic denervation are also essentially different from that observed after adult denervation. While adult muscle fibers atrophied after denervation, denervation during embryogenesis resulted not only in skeletal muscle hypoplasia, but also in "hypertrophy" or "swelling" of individual muscle fibers. Additionally, although structural changes including sarcomere dissolution, endothelial degradation (Oki et al., 1995) and cellular changes such as fewer mitochondria numbers (Rifenberick et al., 1973), accumulation of connective tissue and an abolition in apoptotic myonuclei (Oki et al., 1995) are seen in the adult muscle cell undergoing denervation-induced atrophy, denervation in the embryonic muscle fibers is manifested by a block in terminal differentiation. It can be argued that the lack of sarcomeres in myotubes of $226^{f x D T A} x \mathrm{Olig} 2^{\text {Cre }}$ mice could be caused by degenerative changes leading to sarcomere disassembly. However, sarcomeres never seemed to form in aneural embryonic muscle fibers, as ultrastructural examination of E15.5 muscles showed sparse myofibrils in nascent aneural myotubes and no identifiable definitive sarcomere structure. Moreover, the characteristic striated 
pattern of skeletal muscles was not detected in E14.5 muscle fibers of $R 26^{f x D T A} x \mathrm{Olig} 2 \mathrm{Cre}$ mice by sarcomeric-antibodies.

In the adult, catabolic pathways which partake in neurogenic atrophy, notably the proteasome pathway (ubiquitin-mediated proteolysis) and autophagy (lysosomaldependent degradation) are starting to be elucidated (Beehler et al., 2006; Moresi et al., 2010; Bricceno et al., 2012; O'Leary et al., 2012). In contrast, the precise molecular mechanisms underlying embryonic denervation remain undefined, and it is similarly unclear if they operate in a similar fashion as in the adult. The key feature of adult proteasome pathway controlling neurogenic atrophy is the upregulation of myogenin, an essential regulator of embryonic myogenesis (Moresi et al., 2010). In the postnatal muscle gene regulatory circuit, the lack of innervation causes an upregulation of Hdac4/5 leading to the inhibition of Dach2, thereby permitting myogenin to be transcriptionally active and to bind to E-boxes located upstream of E3 ubiquitin ligase genes such as MuRF1 and Atrogin-1, finally resulting in muscle atrophy (Bodine et al., 2001; Moresi et al., 2010; Macpherson et al., 2011). Gene expression profiles of $R 26^{f x D T A} x O \operatorname{Oig} 2^{\text {Cre }}$ hindlimbs obtained in this study suggest that embryonic denervation operates through pathways differing from the adult denervation pathways, because none of the genes implicated in the proposed pathway, including MAFbx/Atrogin-1 and MuRF1/Trim63 were differentially affected in $R 26^{f x D T A} x \mathrm{Olig} 2^{\mathrm{Cre}}$ mice, while transcripts encoding E3 ubiquitin ligases MuRF1 and Atrogin-1 remained at very low levels in both control and mutant embryos.

Myogenin was identified as having dual roles: being an essential factor in driving myogenic differentiation (Hasty et al., 1993), and participating in neurogenic atrophy (Moresi et al., 2010). Hence, it was unforeseen that its expression was unaffected in the absence of innervation at E15.5. Nevertheless, qPCR analysis of myogenin expression conclusively determined its significant upregulation at late perinatal stages (E18.5), in conjunction with its known reappearance in the muscles as a hallmark of the 
ubiquitin-proteasome pathway in response to postnatal denervation (Moresi et al., 2010; Macpherson et al., 2011). Therefore, while it is evident that embryonic and adult denervation pathway function via diverse effector molecules, the finding that myogenin is only upregulated at late developmental stages could suggest the existence of a transitional time point by late gestation during which the remaining muscle fibers switch to a more adult-like response towards continuing denervation. Additionally, previous studies in adult mice reported that innervation is responsible for downregulating embryonic-type nAChRs (Schuetze and Role, 1987) and adhesion molecules (Covault et al., 1986), leaving the expression of gene encoding musclespecific enzymes or structural proteins such as creatine kinase $(\mathrm{Ckm})$, myosin light chain (Myl) and skeletal $\alpha$-actin (Acta1) wholly unaffected (Eftimie et al., 1991). However, in my study, genes encoding these muscle-specific genes were expressed at significantly lower levels in aneural R26fxDTA $x$ Olig2 ${ }^{C r e}$ muscles. Reduction in Myl2 and Myl3 transcript levels in aneural muscles could already be detected as early as E13.5 (before the onset of muscle loss), thereby excluding the possibility that their downregulation was due to secondary effects caused by muscle degeneration, but rather by a block in terminal differentiation, reflected by their failure to fully express genes encoding certain isoforms of contractile proteins. Taken together, the differences in the response of muscles (i.e., hypertrophy vs. atrophy, sarcomere dissolution vs. terminal differentiation block), signaling pathways involved (excluding apoptosis), time course in activation of caspase 3, as well as the downstream genes towards embryonic and adult denervation underscore the different requirements that embryonic and postnatal muscles may have for innervation.

Finally, since many pathways mediate denervation-induced degeneration in the adults (O'Leary et al., 2012), one relevant question is whether apoptosis is the sole pathway that is activated during embryonic denervation. Hence, it would be worthwhile to determine this by ablating motor neurons in proapoptotic factors Bax and Bak knockout mice which have abolished apoptotic activity and examining if muscle 
hypoplasia can be attenuated or rescued completely under these conditions. Furthermore, microRNA 206 (miRNA 206) has been identified to be implicated in adult musculature circuit in providing positive feedback to Hdac4/5 to promote regeneration (Williams et al., 2009). It is still elusive whether miRNAs are involved in post-transcriptional modifications during embryonic denervation, even though Dicer, an miRNA processing enzyme, has been shown to be involved in embryonic myogenesis (O'Rourke et al., 2007). As such, it would be interesting to screen for embryonic-specific miRNAs that could potentially target some of the positively identified candidate genes or even the non-differentially regulated genes in this study.

\subsection{Working model}

My findings provide conclusive evidence that innervation is dispensable for initiation of both embryonic myotubes, but is absolutely required for the terminal differentiation and maintenance of both primary and secondary myotubes, thus resolving a longstanding controversy regarding the precise role of innervation during embryonic myogenesis. A working model can be proposed according to current results obtained (Figure 4.1). Several permissive cues and effectors could mediate the effects of innervation on the terminal differentiation of myotubes. Interestingly, myoblasts can be induced to differentiate and form sarcomeres autonomously in vitro, in the absence of motor neurons. The difference from the in vivo-situation likely reflects the optimization of these long established protocols for achieving in vitro muscle fiber differentiation with extensive use of combinations of growth factors, which may act in concert to sidestep myotube requirements for innervation in vivo. Transcriptome data showed a set of genes which could reflect the potential myogenic requirements for innervation. These identified factors obtained can be thought of as effectors mediating neural influences to muscles. Together, they may constitute a muscle intrinsic innervation-dependent program exclusive to embryos and serve to turn on the 
transcription of muscle-specific and contractile genes to allow myotubes to be fully functional (i.e., contractile machinery formation, optimized to handle metabolic demands, etc.). Finally, denervation, which perturbs the expression of genes (hence protein activity) implicated in the innervation-dependent program, is hypothesized to cause incomplete terminal differentiation of myotubes, leading to their progressive elimination via apoptosis-mediated degeneration.

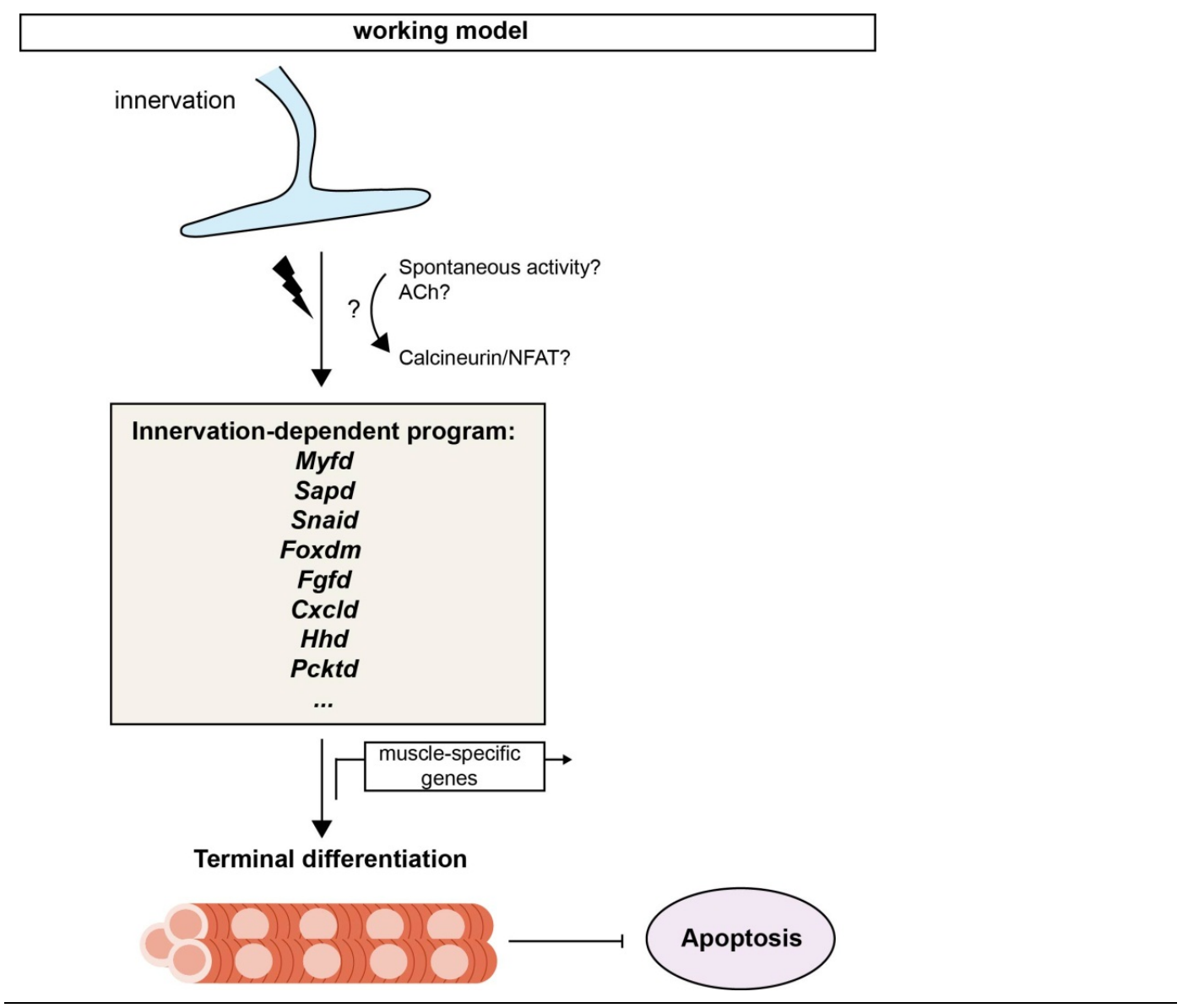

Figure 4.1: Working hypothesis.

Innervation exerts its influence via yet-unidentified pathway(s) and molecules to activate identified candidate genes, which collectively could operate in an embryonic innervation-dependent program. These nerve-dependent genes may act as effectors to turn on transcription of muscle-specific genes, a step required for terminal differentiation to proceed normally. Perturbation in the activation of this program results in the progressive elimination of non-terminally differentiated myotubes via apoptosismediated degeneration. 
On the basis of time-course qPCR analysis, proteins encoded by 'early response genes', especially Myfd and Sapd might be upstream of the signaling pathway to regulate genes affected by denervation. Given evidence that Myfd trancripts are blunted (27\%) in E19 muscles of mice following laser ablation of their lumbosacral spinal cord (Washabaugh et al., 2007), and that Sapd which is localized to the Z-disks of the sarcomere acts as an obligatory calcineurin-anchoring protein in combination with $\alpha$-actinin and calsarcin-1 (Heineke et al., 2005), both proteins may be directly modulated by innervation. Furthermore, drastic reduction in Sapd expression levels was implicated in cardiac myopathy (Heineke et al., 2005), and mutant Sapd heterozygotes showed distinct hypertrophic response with pronounced cardiomyocyte elongation accompanied by reduced NFAT transcriptional activation after induced-myocardial infarction (Heineke et al., 2005), suggesting that Sapd may also be responsible for skeletal muscle defects resulting from motor neuron removal. While functional studies of Snaid are rare, one in vitro study has pointed out to its possible role as a differentiation repressor (Kataoka et al., 2000), contradicting my hypothesis that differentiation is disrupted in the absence of innervation. However, while speculative, the early downregulation of Snaid may reflect the existence of a negative regulatory feedback mechanism after denervation. Fgfd, another 'early response' gene, has both repressive and inductive roles in myogenesis in a dosedependent manner (Armand et al., 2005). Postnatally, a high dose of Fgfd serves to stimulate proliferation of myogenic precursors, whereas at lower doses it modulates muscle differentiation via the calcineurin-signaling pathway (deLapeyriere et al., 1993; Armand et al., 2005). Members of the Fgf family are known to interact with the bHLH MRF proteins; Fgf2 has been shown to limit gene expression of at least myogenin and MyoD in myogenic cultures (Vaidya et al., 1989), and terminal differentiation in Myfdexpressing cells was inhibited in serum-free medium supplemented with basic Fgf (Rhodes and Konieczny, 1989). Since Fgfd transcripts begin to accumulate in myotomes with Myfd simultaneously (Ott et al., 1991; Buckingham et al., 1992), it is 
tempting to hypothesize that (1) both proteins interact with each other as part of the nerve-dependent intricate network consisting of positive and negative regulatory circuits, or (2) via Fgfd modification of specific coregulators that interact with Myfd to control myogenesis during terminal differentiation.

An equally imperative question that warrants further investigation is the mechanisms in the regulation of candidate genes by innervation and the signaling molecules involved. Interesting candidate pathways and molecules that could potentially regulate this intermediary step would be (1) Calcineurin-NFAT pathway and (2) AChmediated spontaneous activity. A large body of work has pointed to myogenic requirements for signals from $\mathrm{Ca}^{2+}$ and $\mathrm{Ca}^{2+}$-dependent pathways (Kegley et al., 2001). Firstly, calcineurin, a $\mathrm{Ca}^{2+}$-calmodulin regulated serine/threonine protein phosphatase and a molecular target of $\mathrm{Ca}^{2+}$, has been documented to partake in MRF gene expression (Friday and Pavlath, 2001), myoblast differentiation (Delling et al., 2000; Friday et al., 2000), hypertrophy (Dunn et al., 1999; Musaro et al., 1999). Secondly, one downstream effector of calcineurin is the nuclear factor of activated T cells (NFAT) which, when dephosphorylated by calcineurin, translocates from the cytoplasm to the nucleus where it acts as a transcription factor in combination with Mef2 or Gata2 in skeletal muscles (Dolmetsch et al., 1997; Chin et al., 1998; Musaro et al., 1999). Various NFAT isoforms (c1, c2 and c3) are expressed specifically in the muscles; inactivating Nfatc2 in mice resulted in fiber type abnormalities and reduced muscle size (Horsley et al., 2001), whereas inactivating Nfatc3 led to reduced myofiber numbers hence decreased muscle mass (Kegley et al., 2001). Even though genes encoding calcineurin and NFAT were not differentially expressed between control and aneural $R 26^{f x D T A} x O \operatorname{Oig} 2^{\text {Cre }}$ muscles, posttranslational modifications and activity level in the two effectors might be responsible for differences in downstream target gene expression (possibly calcineurin- or NFAT-dependent). This could perhaps involve the binding of NFAT to the enhancer/promoter regions of candidate genes, or the recruitment of coactivators to enhance transcription of candidate genes through combinatorial 
regulation. One such co-activator might be Mef2, known to be de-repressed by NFAT. Additionally, a high expression level of Myf5 (member of MRF) could be induced via calcineurin-NFAT signaling pathways in primary myoblast cells treated with ionomycin (a bacterial ionophore used to increase intracellular $\mathrm{Ca}^{2+}$ levels) (Friday et al., 2000; Perez-Ruiz et al., 2007). Together, previous studies indicate that there is a direct interaction between calcineurin pathway, MRF expression and skeletal muscle differentiation (Al-Shanti and Stewart, 2009). Therefore, it is important to examine if components in $\mathrm{Ca}^{2+}$-dependent pathways are differentially affected by the removal of motor neurons at the protein level in order to uncover the causative link between the lack of innervation, downregulation of candidate genes and finally terminal differentiation failure in aneural muscles. Likewise, the possible importance of ACh release especially during spontaneous activity that could serve as an important trigger for downstream signaling pathway cannot be undermined, and has to be addressed with conditional motor neuron Chat knockout mice. 


\section{Outlook}

\subsection{In ovo electroporation as a screening platform for innnervation-dependent myogenic candidate genes}

Even though I identified a set of candidate genes which could potentially link nerve influence to the promotion of myogenesis, a challenging yet essential task will be to explore their functions during embryonic skeletal muscle development in the presence and absence of the nerve. Addressing this exclusively through gain- or loss-of-function studies in rodents for all genes of interest is largely precluded by the limited availability of mouse lines, as well as the cost, time, labor and resource-consuming nature of establishing mouse genetic models for each candidate gene. Another issue could be complications arising from conventional gene targeting studies in mice. For instance, attempts at uncovering the context of gene activation by MRFs by using gene targeting strategies in rodents were complicated by the cis interaction between closely linked genes on a chromosome, such that deletion of one gene would inherently affect the expression of the other gene (Olson et al., 1996). Therefore, another alternative screening platform is compulsory for addressing the role of innervation-dependent candidate genes for embryonic myogenesis.

As a choice model, screening procedures for suitable candidate genes can be shifted to the the chick embryo (Gallus gallus domesticus). Electroporation is a widely-used technique for transfecting neural and mesodermal tissues in chick embryos (Wang et al., 2011a), and studies utilizing the somite and neural tube electroporation technique have also been published (Delfini and Duprez, 2004; Gros et al., 2004; FournierThibault et al., 2009). The chick shares many conserved features regulating developmental processes and shows extensive homology in the organization of the nervous and skeletal muscle systems with mammals (Uchikawa, 2008), including highly conserved myogenic programmes operating during mammalian and avian 
development (Mok and Sweetman, 2011), thereby making in ovo electroporation a suitable as well as a time- and cost-efficient method for rapid gene transfer to muscles, and an alternative to rodent transgenics. Preliminary results obtained via chick somite electroporation using transposon-based plasmid constructs showed highly effective stable and unbiased gene transfer into limb muscle cells of late-gestation chick embryos, as confirmed by robust reporter gene expression in muscle fibers (Figure 5.1).

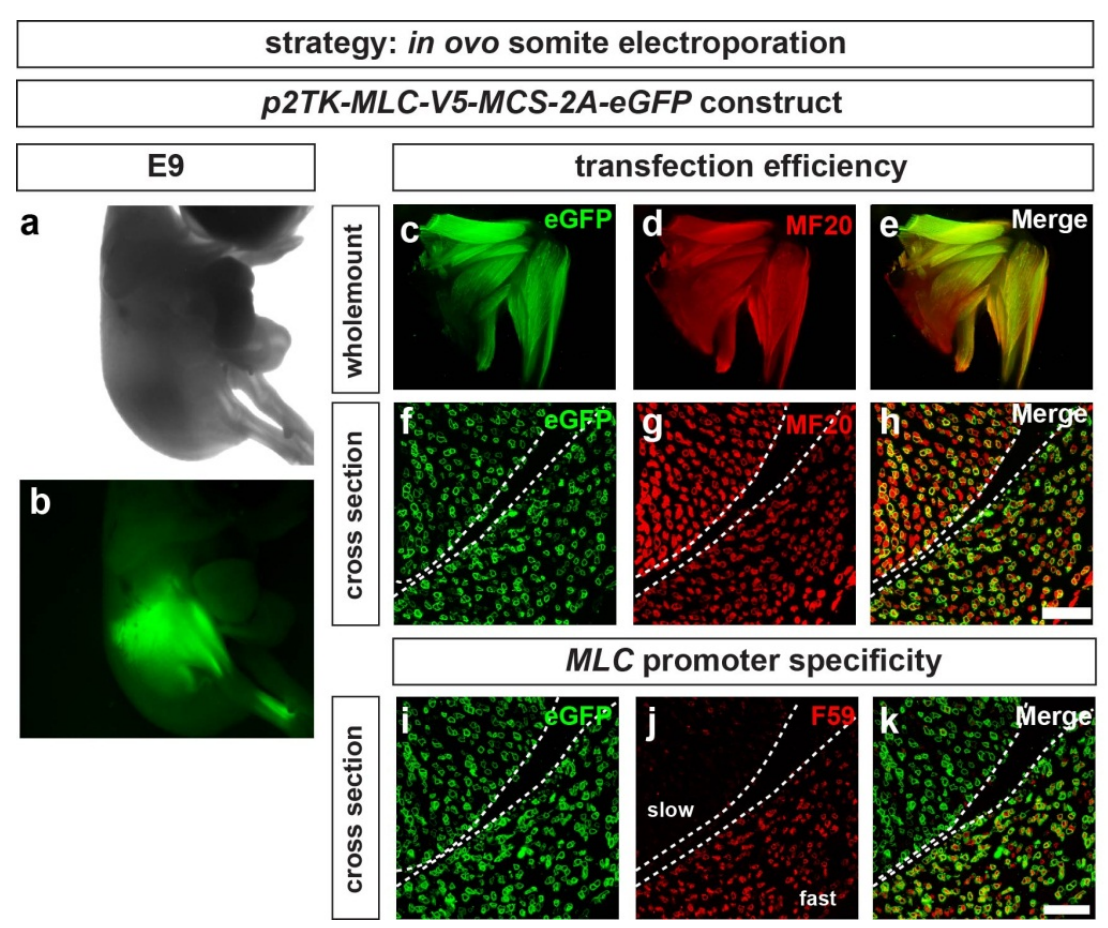

Figure 5.1: A screening platform for candidate gene function.

(a-b) E9 chick embryo with (a) brightfield and (b) fluorescence illumination. Robust eGFP expression is specific to hindlimb of chick embryo. (c-h) Immunostaining of (c-e) whole mount and (f-h) transverse section of E9 chick hindlimb with MF20 and GFP antibodies. Transfected muscle fibers colocalized with $\mathrm{MF}^{2} \mathrm{0}^{+}$muscle fibers demonstrating a high transfection efficiency. (i-k) Fast myosin antibody revealed that $M L C 1 F / 3 F$ promoter is not specfic to any specfic type of muscles, but drives ectopic gene expression in all muscle fibers. Muscle fibers which were negative for fast myosin were also transfected and expressed eGFP. Dotted line demarcate slow and fast muscles. MF20, pan muscle fiber marker; F59, fast myosin marker. All scale bars: $50 \mu \mathrm{m}$. 
To analyze gene function in the context of denervation and to circumvent using manual dissection or pharmacological applications in chick embryos, rescue experiments making use of the in ovo electroporation of the neural tube and somite technique can be performed by using the constructs listed in Figure $5.2 \mathrm{a}$.

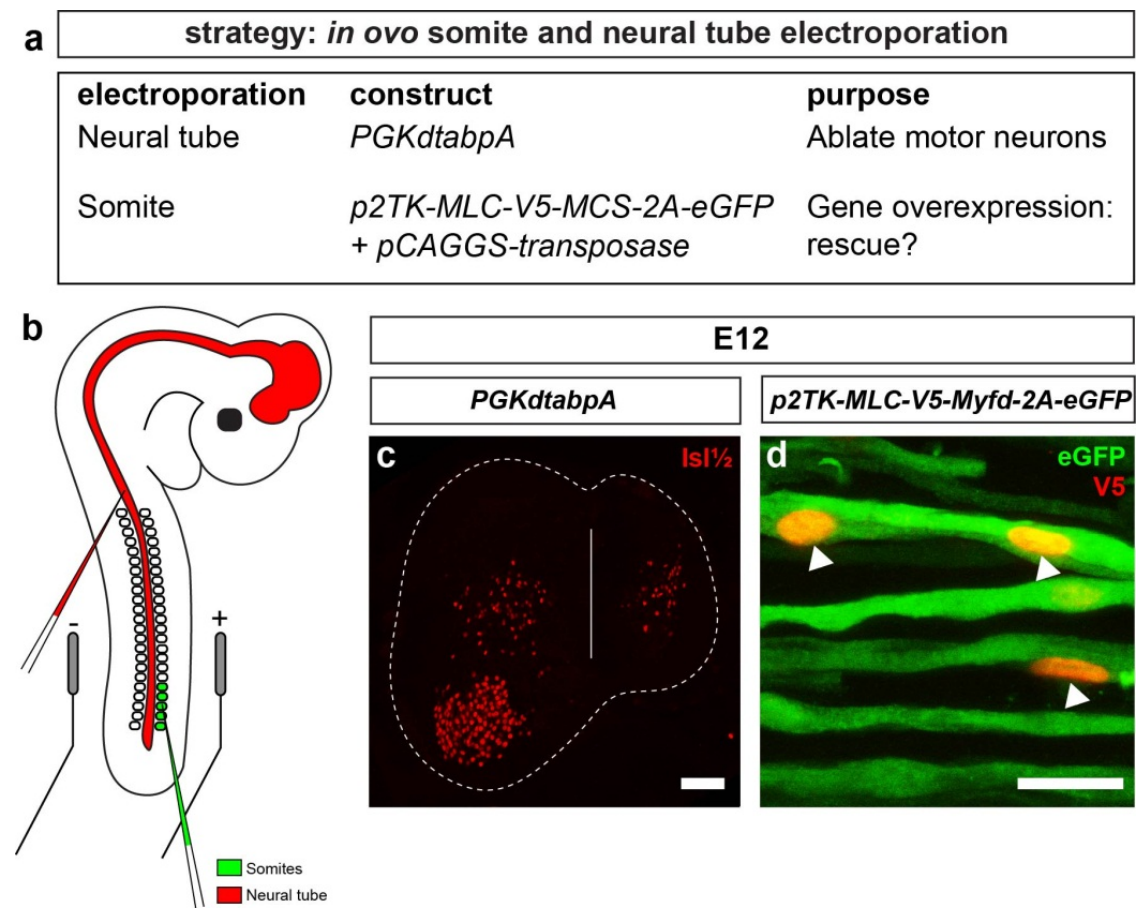

Figure 5.2: Strategy to study candidate gene function in context of denervation.

(a-b) To ablate motor neurons and overexpress candidate genes in muscles for rescue experiments in chick embryo, (a) different vectors for in ovo neural tube and somite electroporation were used. (b) Schematic of site of injection and positioning of electrodes. (c) Isl $1 / 2^{+}$motor neurons were absent from the transfected side of the atrophied spinal cord. Dotted line demarcates border and bold line marks midline of spinal cord. (d) High magnification view of transfected longitudinal muscle fibers immunostained with GFP and V5-tag epitope. Ectopic Myfd expression was localized to myonuclei indicated by arrowheads, in consistent to its role as a transcription factor. All scale bars: $20 \mu \mathrm{m}$.

Methodologically, limb somites can be co-injected with transposon vectors driving expression e.g. of Myfd as the gene of interest. To genetically ablate motor neurons in chick embryos, the neural tube lumen can be injected and transfected into neural progenitor cells via in ovo electroporation with constructs driving expression of DTA. 
Thereafter, the chick embryo is placed between two platinum electrodes, followed by electroporation (Figure 5.2 b). Successful removal of motor neurons can be assessed by the atrophy of the ventral horn (lamina IX) on the transfected side of the spinal cord and the absence of Isl $12^{+}$cells in the ventral part of the spinal cord (Figure $5.2 \mathrm{c}$ ). Similarly, successful ectopic expression of transgene in muscle fibers can also be determined by immunostaining with V5-epitope tag and GFP antibodies (Figure 5.2 d). Taken together, this method is applicable for future bioassay exploring the functions of candidate genes which have been obtained in this study.

\subsection{Spinal motor neuron dependence on target muscles}

Although this project is dedicated to studying the dependence of muscles on their innervating motor neurons during embryogenesis, it is also inherently linked to the converse dependence of motor neuron survival on retrograde signals provided by their peripheral target muscles. This is indeed one of the oldest paradigms in developmental neurobiology, which was based on the observation that motor neurons die after chick limb removal during early embryogenesis (Oppenheim, 1991). During embryonic development, almost half of the motor neurons which were generated readily undergo PCD (programmed cell death), and this event coincides with the initiation of synaptogenesis between motor nerve terminals and muscle cells (Hamburger and Levi-Montalcini, 1949; Hamburger, 1975; Oppenheim, 1991). The death of motor neurons has been hypothesized to reflect their failure to obtain a sufficient amount of neurotrophic factors (Oppenheim, 1989; Snider and Johnson, 1989). This hypothesis has been studied extensively and much effort has been undertaken to identify skeletal muscle-derived neurotrophic factors which contribute to the survival of developing motor neurons in the spinal cord. A more recent study used knockout mice lacking MRF members, Myf5 and MyoD (known muscle cell determinants) (Berkes and Tapscott, 2005), in which muscle precursor cells are formed 
(Kablar et al., 2003), but fail to differentiate into myoblasts and myotubes (Rudnicki et al., 1993; Kablar and Rudnicki, 1999; Kablar et al., 2003), resulting in mouse embryos that are from the outset amyogenic. Through their study they found that motor neurons are progressively lost from the spinal cord of Myf5-/-;MyoD-/- mice during gestation, and attributed this to the lack of trophic support from muscle normally providing neurotrophic factors Bdnf, Nt3, Nt4/5 and Gdnf (Kablar and Belliveau, 2005). Since these studies only analyzed spinal cord sections from E13.5 mice and not later in development, potentially altered molecular programs of motor neurons devoid of muscle-derived neurotrophic factors or other molecules were not examined. Hence, it will be highly interesting to re-examine the prerequisites for motor neuron survival and identify possible regulatory factors involved in this process, which may provide novel insights into extrinsic cues driving motor neuron survival that may profoundly contribute to our understanding of human pathological conditions in which motor neurons degenerate.

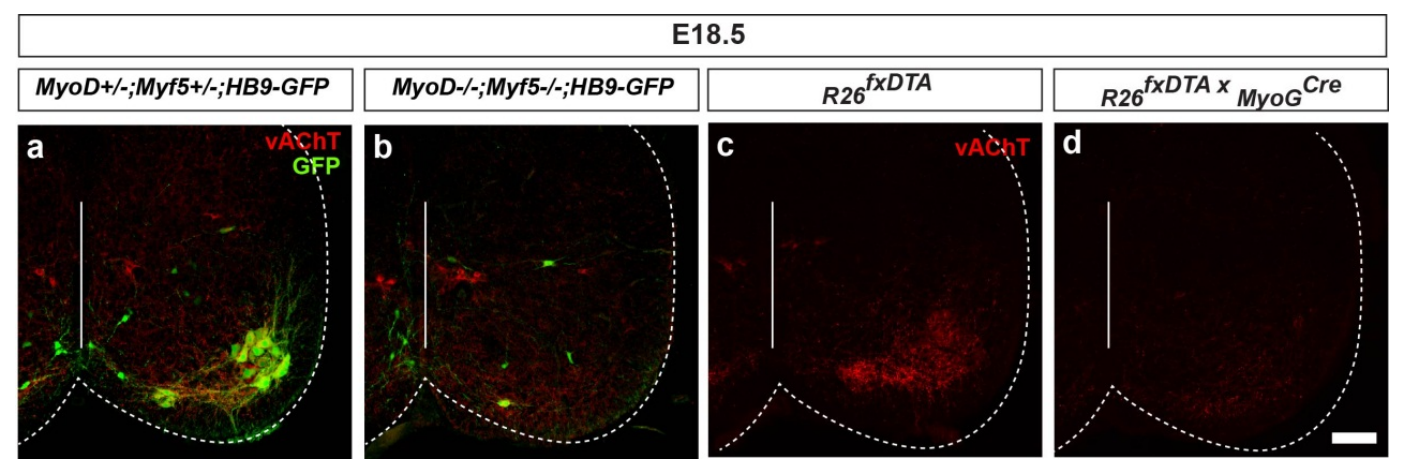

Figure 5.3: Different mouse lines to genetically abolish myogenesis.

(a-b) E18.5 transverse lumbar spinal cord sections of (a) heterozygous control and (b) double knockout mice. Mice were crossed with HB9-GFP mice to genetically label motor neurons (green). Mutant mice are amyogenic because their muscle precursor cells failed to undergo specification and differentiation. (c-d) E18.5 transverse lumbar spinal cord sections of (c) control and (d) R26 $6^{f x T A} x M y o G^{c r e}$ mice. Specified muscle cells were specifically ablated in mutant mice, thereby blocking further differentiation and resulting in amyogenic mice. In both mutant mouse lines, the lack of target muscles led to the complete elimination of spinal motor neurons at E18.5. Dotted line demarcate border of spinal cord. Scale bar: 100 $\mu \mathrm{m}$. 
Preliminary results obtained from Myf5+/-;MyoD+/+ and Myf5-/-;MyoD-/- mice crossed with HB9-GFP mice to genetically label motor neurons showed that motor neurons were completely eliminated by E18.5 in the double knockout mutants (Figure $5.3 \mathrm{a}-\mathrm{b}$ ), thereby confirming that motor neurons die in the absence of target muscles. Genetically labeling motor neurons with GFP enables better visualization of motor neurons and facilitates the identification of motor neuron columns, which can thereby be isolated and processed for transcriptome profiling to uncover genetically encoded factors potentially functioning as effectors of muscle-derived survival factors. Alternatively, $R 26^{f x D T A}$ mice crossed with $M y o G^{\text {cre }}$ mice to yield amyogenic $R 26^{f x D T A} x M y o G^{C r e}$ mutant embryos may also be used for transcriptome profiling. This would provide the advantage of a simpler, less time-consuming breeding scheme and the higher probability of obtaining embryos with desired genotypes, compared to Myf5-/-;MyoD-/- mice (Rudnicki et al., 1993). Muscle precursor cells in R26 fxDTA $x M y o G^{C r e}$ mice are specified, but fail to differentiate (Gensch et al., 2008). However, consistent with findings by Gensch et al. (2008), efficient killing of muscle cells in $R 26^{f x D T A} x M y o G^{C r e}$ mice was not detected before E14.5 (data not shown). This was attributed to a delay between the activation of promoter activity driving Crerecombinase expression, Cre-mediated recombination of DTA locus and ablation of muscle cells in $R 26^{f x D T A} x M y o G^{C r e}$ mice (Gensch et al., 2008). Despite the delayed ablation of muscles, I found that $R 26^{f x D T A} x M y o G^{C r e}$ mutants were completely devoid of motor neurons by E18.5 (Figure $5.3 \mathrm{c}-\mathrm{d}$ ), similar to Myf5-/-;MyoD-/-;HB9-GFP mice. Thus, motor neurons critically depend on continuing support by muscle-derived factors for their survival and further development during embryogenesis, providing an entry point for identifying novel genetically encoded factors underlying motor neuron survival. 


\section{Summary}

The interdependency of motor neurons and the skeletal muscle is a classical example for instructive and trophic tissue interactions during development and adult life. It is well established that skeletal muscles undergo atrophy in the absence of motor neuron innervation. In the adult, molecular pathways underlying the dependency of muscle fibers on innervation have begun to emerge. However, it is much less clear how and to what extent motor neuronal input contributes to myogenesis during embryonic development. Previous studies using manual dissection and systemic application of pharmacological paralyzing agents have led to two main yet divergent conclusions on the myogenic requirements from the nerve as well as the myogenic response to denervation.

The aim of my thesis project was to resolve these issues through selective gene manipulation in the mouse. Targeted genetic suppression of motor neuron generation, leaving skeletal muscle from the outset devoid of motor innervation, did not affect the formation of primary or secondary myotubes. At the same time, however, the absence of motor innervation completely prevented terminal differentiation of myotubes into functionally contractile fibers and resulted in their progressive apoptotic elimination. This in turn led to a compensatory proliferation of satellite cells to make up for muscle fiber loss. The impacts caused by wholesale removal of motor innervation were faithfully phenocopied in the absence of cholinergic neuromuscular transmission. On the other hand, milder skeletal muscle hypoplasia was observed after selective genetic suppression of evoked, but not spontaneous neuromuscular transmission. Transcriptome profiling revealed a unique immediate early response of myotubes lacking motor innervation that differed from the pathways operating during adult denervation. Herein, embryonic innervation appears to operate through a dedicated set of myogenic transcription factors previously implicated in muscle fiber terminal 
differentiation. Taken together, my studies provide conclusive evidence that motor innervation is dispensable for the principal formation of both primary and secondary myotubes, but is absolutely required for their terminal differentiation into contractile fibers and subsequent survival, thus resolving a long-standing controversy surrounding the role of innervation in skeletal myogenesis. I further found that developmental and adult innervation operate through distinct molecular pathways to promote muscle fiber differentiation and survival, and identified a candidate myogenic program which may underlie innervation-dependent embryonic myogenesis. 


\section{References}

Abmayr, S. M. and Pavlath, G. K. (2012) 'Myoblast fusion: lessons from flies and mice', Development 139(4): 641-56.

Adams, L., Carlson, B. M., Henderson, L. and Goldman, D. (1995) 'Adaptation of nicotinic acetylcholine receptor, myogenin, and MRF4 gene expression to long-term muscle denervation', J Cell Biol 131(5): 1341-9.

Al-Shanti, N. and Stewart, C. E. (2009) 'Ca2+/calmodulin-dependent transcriptional pathways: potential mediators of skeletal muscle growth and development', Biol Rev Camb Philos Soc 84(4): 637-52.

Altar, C. A. and DiStefano, P. S. (1998) 'Neurotrophin trafficking by anterograde transport', Trends Neurosci 21(10): 433-7.

Amthor, H., Christ, B. and Patel, K. (1999) 'A molecular mechanism enabling continuous embryonic muscle growth - a balance between proliferation and differentiation', Development 126(5): 1041-53.

Arber, S., Han, B., Mendelsohn, M., Smith, M., Jessell, T. M. and Sockanathan, S. (1999) 'Requirement for the homeobox gene $\mathrm{Hb} 9$ in the consolidation of motor neuron identity', Neuron 23(4): 659-74.

Armand, A. S., Pariset, C., Laziz, I., Launay, T., Fiore, F., Della Gaspera, B., Birnbaum, D., Charbonnier, F. and Chanoine, C. (2005) 'FGF6 regulates muscle differentiation through a calcineurin-dependent pathway in regenerating soleus of adult mice', J Cell Physiol 204(1): 297-308.

Ashby, P. R., Pincon-Raymond, M. and Harris, A. J. (1993a) 'Regulation of myogenesis in paralyzed muscles in the mouse mutants peroneal muscular atrophy and muscular dysgenesis', Dev Biol 156(2): 529-36.

Ashby, P. R., Wilson, S. J. and Harris, A. J. (1993b) 'Formation of primary and secondary myotubes in aneural muscles in the mouse mutant peroneal muscular atrophy', Dev Biol 156(2): 519-28. 
Axelsson, J. and Thesleff, S. (1959) 'A study of supersensitivity in denervated mammalian skeletal muscle', J Physiol 147(1): 178-93.

Barbieri, G., De Angelis, L., Feo, S., Cossu, G. and Giallongo, A. (1990) 'Differential expression of muscle-specific enolase in embryonic and fetal myogenic cells during mouse development', Differentiation 45(3): 179-84.

Beehler, B. C., Sleph, P. G., Benmassaoud, L. and Grover, G. J. (2006) 'Reduction of skeletal muscle atrophy by a proteasome inhibitor in a rat model of denervation', Exp Biol Med (Maywood) 231(3): 335-41.

Bentzinger, C. F., Wang, Y. X. and Rudnicki, M. A. (2012) 'Building muscle: molecular regulation of myogenesis', Cold Spring Harb Perspect Biol 4(2).

Berchtold, M. W., Brinkmeier, H. and Muntener, M. (2000) 'Calcium ion in skeletal muscle: its crucial role for muscle function, plasticity, and disease', Physiol Rev 80(3): 1215-65.

Berkes, C. A. and Tapscott, S. J. (2005) 'MyoD and the transcriptional control of myogenesis', Semin Cell Dev Biol 16(4-5): 585-95.

Bernheim, L., Liu, J. H., Hamann, M., Haenggeli, C. A., Fischer-Lougheed, J. and Bader, C. R. (1996) 'Contribution of a non-inactivating potassium current to the resting membrane potential of fusion-competent human myoblasts', J Physiol 493 ( Pt 1): 12941.

Biressi, S., Molinaro, M. and Cossu, G. (2007) 'Cellular heterogeneity during vertebrate skeletal muscle development', Dev Biol 308(2): 281-93.

Bismuth, K. and Relaix, F. (2010) 'Genetic regulation of skeletal muscle development', Exp Cell Res 316(18): 3081-6.

Black, B. L. and Olson, E. N. (1998) 'Transcriptional control of muscle development by myocyte enhancer factor-2 (MEF2) proteins', Annu Rev Cell Dev Biol 14: 167-96.

Bladt, F., D, R., S, I., A, A. and C., B. (1995) 'Essential role for the c-met receptor in the migration of myogenic precursor cells into the limb bud.', Nature 31(376): 768-71. 
Bloom, J. W., Butler, J., Brierley, J. and Cosmos, E. (1985) 'Direct electrical stimulation promotes growth and enhances survival of aneurogenic muscles of the chick embryo', J Neurosci 5(2): 414-20.

Bodine, S. C., Latres, E., Baumhueter, S., Lai, V. K., Nunez, L., Clarke, B. A., Poueymirou, W. T., Panaro, F. J., Na, E., Dharmarajan, K. et al. (2001) 'Identification of ubiquitin ligases required for skeletal muscle atrophy', Science 294(5547): 1704-8.

Bonanomi, D. and Pfaff, S. L. (2010) 'Motor axon pathfinding', Cold Spring Harb Perspect Biol 2(3): a001735.

Bonner, P. H. and Hauschka, S. D. (1974) 'Clonal analysis of vertebrate myogenesis. I. Early developmental events in the chick limb', Dev Biol 37(2): 317-28.

Boonyarom, O. and Inui, K. (2006) 'Atrophy and hypertrophy of skeletal muscles: structural and functional aspects', Acta Physiol (Oxf) 188(2): 77-89.

Borisov, A. B., Dedkov, E. I. and Carlson, B. M. (2001) 'Interrelations of myogenic response, progressive atrophy of muscle fibers, and cell death in denervated skeletal muscle', Anat Rec 264(2): 203-18.

Borisov, A. B., Dedkov, E. I. and Carlson, B. M. (2005) 'Abortive myogenesis in denervated skeletal muscle: differentiative properties of satellite cells, their migration, and block of terminal differentiation', Anat Embryol (Berl) 209(4): 269-79.

Borycki, A., B, B., S, T., M, B., C, C. and Jr., E. C. (1999) 'Sonic hedgehog controls epaxial muscle determination through Myf5 activation.', Development 126(18): 4053-63.

Borycki, A. and Emerson, C. (1997) 'Muscle determination: another key player in myogenesis?', Curr Biol. 7(10): 620-3.

Bradley, S. J. (1970) 'An analysis of self-differentiation of chick limb buds in chorioallantoic grafts', J Anat 107(Pt 3): 479-90.

Brandon, E. P., Lin, W., D'Amour, K. A., Pizzo, D. P., Dominguez, B., Sugiura, Y., Thode, S., Ko, C. P., Thal, L. J., Gage, F. H. et al. (2003) 'Aberrant patterning of neuromuscular synapses in choline acetyltransferase-deficient mice', J Neurosci 23(2): $539-49$. 
Braun, T. and Arnold, H. H. (1995) 'Inactivation of Myf-6 and Myf-5 genes in mice leads to alterations in skeletal muscle development', EMBO J 14(6): 1176-86.

Braun, T. and Gautel, M. (2011) 'Transcriptional mechanisms regulating skeletal muscle differentiation, growth and homeostasis', Nat Rev Mol Cell Biol 12(6): 349-61.

Braun, T., Rudnicki, M. A., Arnold, H. H. and Jaenisch, R. (1992) 'Targeted inactivation of the muscle regulatory gene Myf-5 results in abnormal rib development and perinatal death', Cell 71(3): 369-82.

Breitman, M. L., Rombola, H., Maxwell, I. H., Klintworth, G. K. and Bernstein, A. (1990) 'Genetic ablation in transgenic mice with an attenuated diphtheria toxin A gene', Mol Cell Biol 10(2): 474-9.

Brennan, T. J. and Olson, E. N. (1990) 'Myogenin resides in the nucleus and acquires high affinity for a conserved enhancer element on heterodimerization', Genes Dev 4(4): $582-95$.

Brent, A. E. and Tabin, C. J. (2002) 'Developmental regulation of somite derivatives: muscle, cartilage and tendon', Curr Opin Genet Dev 12(5): 548-57.

Bricceno, K. V., Sampognaro, P. J., Van Meerbeke, J. P., Sumner, C. J., Fischbeck, K. H. and Burnett, B. G. (2012) 'Histone deacetylase inhibition suppresses myogenindependent atrogene activation in spinal muscular atrophy mice', Hum Mol Genet 21(20): 4448-59.

Briscoe, J., Pierani, A., Jessell, T. M. and Ericson, J. (2000) 'A homeodomain protein code specifies progenitor cell identity and neuronal fate in the ventral neural tube', Cell 101(4): 435-45.

Broadie, K., Prokop, A., Bellen, H. J., O'Kane, C. J., Schulze, K. L. and Sweeney, S. T. (1995) 'Syntaxin and synaptobrevin function downstream of vesicle docking in Drosophila', Neuron 15(3): 663-73.

Bryson-Richardson, R. J. and Currie, P. D. (2008) 'The genetics of vertebrate myogenesis', Nat Rev Genet 9(8): 632-46. 
Buckingham, M., Bajard, L., Chang, T., Daubas, P., Hadchouel, J., Meilhac, S., Montarras, D., Rocancourt, D. and Relaix, F. (2003) 'The formation of skeletal muscle: from somite to limb', J Anat 202(1): 59-68.

Buckingham, M., Houzelstein, D., Lyons, G., Ontell, M., Ott, M. O. and Sassoon, D. (1992) 'Expression of muscle genes in the mouse embryo', Symp Soc Exp Biol 46: 203-17. Buller, A. J., Eccles, J. C. and Eccles, R. M. (1960) 'Interactions between motoneurones and muscles in respect of the characteristic speeds of their responses', J Physiol 150: 417-39.

Butler, J., Cosmos, E. and Brierley, J. (1982) 'Differentiation of muscle fiber types in aneurogenic brachial muscles of the chick embryo', J Exp Zool 224(1): 65-80.

Cai, J., Xu, X., Yin, H., Wu, R., Modderman, G., Chen, Y., Jensen, J., Hui, C. C. and Qiu, M. (2000) 'Evidence for the differential regulation of Nkx-6.1 expression in the ventral spinal cord and foregut by Shh-dependent and -independent mechanisms', Genesis 27(1): 6-11.

Caldero, J., Prevette, D., Mei, X., Oakley, R. A., Li, L., Milligan, C., Houenou, L., Burek, M. and Oppenheim, R. W. (1998) 'Peripheral target regulation of the development and survival of spinal sensory and motor neurons in the chick embryo', J Neurosci 18(1): $356-70$.

Chevrel, G., Hohlfeld, R. and Sendtner, M. (2006) 'The role of neurotrophins in muscle under physiological and pathological conditions', Muscle Nerve 33(4): 462-76.

Chin, E. R., Olson, E. N., Richardson, J. A., Yang, Q., Humphries, C., Shelton, J. M., Wu, H., Zhu, W., Bassel-Duby, R. and Williams, R. S. (1998) 'A calcineurin-dependent transcriptional pathway controls skeletal muscle fiber type', Genes Dev 12(16): 2499509.

Christ, B., Brand-Saberi, B., Grim, M. and Wilting, J. (1992) 'Local signalling in dermomyotomal cell type specification', Anat Embryol (Berl) 186(5): 505-10.

Christ, B. and Ordahl, C. P. (1995) 'Early stages of chick somite development', Anat Embryol (Berl) 191(5): 381-96. 
Cohen, T. J., Waddell, D. S., Barrientos, T., Lu, Z., Feng, G., Cox, G. A., Bodine, S. C. and Yao, T. P. (2007) 'The histone deacetylase HDAC4 connects neural activity to muscle transcriptional reprogramming', J Biol Chem 282(46): 33752-9.

Condon, K., Silberstein, L., Blau, H. M. and Thompson, W. J. (1990) 'Differentiation of fiber types in aneural musculature of the prenatal rat hindlimb', Dev Biol 138(2): 27595.

Connold, A. L., Kamel-Reid, S., Vrbova, G. and Zak, R. (1993) 'Inactivity induces muscle hypertrophy and redistribution of myosin isozymes in chicken anterior latissimus dorsi muscle', Pflugers Arch 423(1-2): 34-40.

Cossu, G. and Borello, U. (1999) 'Wnt signaling and the activation of myogenesis in mammals', EMBO J 18(24): 6867-72.

Covault, J., Merlie, J. P., Goridis, C. and Sanes, J. R. (1986) 'Molecular forms of N-CAM and its RNA in developing and denervated skeletal muscle', J Cell Biol 102(3): 731-9.

Cowling, B. S., McGrath, M. J., Nguyen, M. A., Cottle, D. L., Kee, A. J., Brown, S., Schessl, J., Zou, Y., Joya, J., Bonnemann, C. G. et al. (2008) 'Identification of FHL1 as a regulator of skeletal muscle mass: implications for human myopathy', J Cell Biol 183(6): 1033-48.

Creazzo, T. L. and Sohal, G. S. (1981) 'Beta-bungarotoxin lacking phospholipase activity is not toxic to developing motor neurons and skeletal muscle', Dev Neurosci 4(5): 329-36.

Creazzo, T. L. and Sohal, G. S. (1983) 'Neural control of embryonic acetylcholine receptor and skeletal muscle', Cell Tissue Res 228(1): 1-12.

Crow, M. T. and Stockdale, F. E. (1986) 'The developmental program of fast myosin heavy chain expression in avian skeletal muscles', Dev Biol 118(2): 333-42.

Dahm, L. M. and Landmesser, L. T. (1988) 'The regulation of intramuscular nerve branching during normal development and following activity blockade', Dev Biol 130(2): 621-44. 
Dahm, L. M. and Landmesser, L. T. (1991) 'The regulation of synaptogenesis during normal development and following activity blockade', J Neurosci 11(1): 238-55.

Dasen, J. S. and Jessell, T. M. (2009) 'Hox networks and the origins of motor neuron diversity', Curr Top Dev Biol 88: 169-200.

Deitcher, D. L., Ueda, A., Stewart, B. A., Burgess, R. W., Kidokoro, Y. and Schwarz, T. L. (1998) 'Distinct requirements for evoked and spontaneous release of neurotransmitter are revealed by mutations in the Drosophila gene neuronalsynaptobrevin', J Neurosci 18(6): 2028-39.

deLapeyriere, O., Ollendorff, V., Planche, J., Ott, M. O., Pizette, S., Coulier, F. and Birnbaum, D. (1993) 'Expression of the Fgf6 gene is restricted to developing skeletal muscle in the mouse embryo', Development 118(2): 601-11.

Delfini, M. C. and Duprez, D. (2004) 'Ectopic Myf5 or MyoD prevents the neuronal differentiation program in addition to inducing skeletal muscle differentiation, in the chick neural tube', Development 131(4): 713-23.

Delling, U., Tureckova, J., Lim, H. W., De Windt, L. J., Rotwein, P. and Molkentin, J. D. (2000) 'A calcineurin-NFATc3-dependent pathway regulates skeletal muscle differentiation and slow myosin heavy-chain expression', Mol Cell Biol 20(17): 6600-11. Dietrich, S., Abou-Rebyeh, F., Brohmann, H., Bladt, F., Sonnenberg-Riethmacher, E., Yamaai, T., Lumsden, A., Brand-Saberi, B. and Birchmeier, C. (1999) 'The role of SF/HGF and c-Met in the development of skeletal muscle', Development 126(8): 1621-9.

Dolmetsch, R. E., Lewis, R. S., Goodnow, C. C. and Healy, J. I. (1997) 'Differential activation of transcription factors induced by $\mathrm{Ca} 2+$ response amplitude and duration', Nature 386(6627): 855-8.

Drachman, D. B. (1964) 'Atrophy of Skeletal Muscle in Chick Embryos Treated with Botulinum Toxin', Science 145(3633): 719-21.

Drachman, D. B. (1967) 'Is acetylcholine the trophic neuromuscular transmitter?', Arch Neurol 17(2): 206-18. 
Drachman, D. B. and Coulombre, A. J. (1962) 'Method for continuous infusion of fluids into the chorioallantoic circulation of the chick embryo', Science 138(3537): 144-5.

Dunn, S. E., Burns, J. L. and Michel, R. N. (1999) 'Calcineurin is required for skeletal muscle hypertrophy', J Biol Chem 274(31): 21908-12.

Duxson, M. J. (1982) 'The effect of postsynaptic block on development of the neuromuscular junction in postnatal rats', J Neurocytol 11(3): 395-408.

Duxson, M. J., Usson, Y. and Harris, A. J. (1989) 'The origin of secondary myotubes in mammalian skeletal muscles: ultrastructural studies', Development 107(4): 743-50.

Edmondson, D. G. and Olson, E. N. (1989) 'A gene with homology to the myc similarity region of MyoD1 is expressed during myogenesis and is sufficient to activate the muscle differentiation program', Genes Dev 3(5): 628-40.

Eftimie, R., Brenner, H. R. and Buonanno, A. (1991) 'Myogenin and MyoD join a family of skeletal muscle genes regulated by electrical activity', Proc Natl Acad Sci U S A 88(4): 1349-53.

Entwistle, A., Zalin, R. J., Warner, A. E. and Bevan, S. (1988) 'A role for acetylcholine receptors in the fusion of chick myoblasts', J Cell Biol 106(5): 1703-12.

Fatt, P. and Katz, B. (1952) 'Spontaneous subthreshold activity at motor nerve endings', J Physiol 117: 109-128.

Feng, T. P. and Lu, D. X. (1965) 'New lights on the phenomenon of transient hypertrophy in the denervated hemidiaphragm of the rat', Sci Sin 14(12): 1772-84.

Ferrari, M., Binzoni, T. and Quaresima, V. (1997) 'Oxidative metabolism in muscle', Philos Trans R Soc Lond B Biol Sci 352(1354): 677-83.

Ferrari, M. B., Ribbeck, K., Hagler, D. J. and Spitzer, N. C. (1998) 'A calcium signaling cascade essential for myosin thick filament assembly in Xenopus myocytes', J Cell Biol 141(6): 1349-56.

Ferrari, M. B., Rohrbough, J. and Spitzer, N. C. (1996) 'Spontaneous calcium transients regulate myofibrillogenesis in embryonic Xenopus myocytes', Dev Biol 178(2): 484-97. 
Fischer-Lougheed, J., Liu, J. H., Espinos, E., Mordasini, D., Bader, C. R., Belin, D. and Bernheim, L. (2001) 'Human myoblast fusion requires expression of functional inward rectifier Kir2.1 channels', J Cell Biol 153(4): 677-86.

Flucher, B. E. and Andrews, S. B. (1993) 'Characterization of spontaneous and action potential-induced calcium transients in developing myotubes in vitro', Cell Motil Cytoskeleton 25(2): 143-57.

Fougerousse, F., Edom-Vovard, F., Merkulova, T., Ott, M. O., Durand, M., ButlerBrowne, G. and Keller, A. (2001) 'The muscle-specific enolase is an early marker of human myogenesis', J Muscle Res Cell Motil 22(6): 535-44.

Fournier-Thibault, C., Blavet, C., Jarov, A., Bajanca, F., Thorsteinsdottir, S. and Duband, J. L. (2009) 'Sonic hedgehog regulates integrin activity, cadherin contacts, and cell polarity to orchestrate neural tube morphogenesis', J Neurosci 29(40): 12506-20.

Fredette, B. J. and Landmesser, L. T. (1991a) 'A reevaluation of the role of innervation in primary and secondary myogenesis in developing chick muscle', Dev Biol 143(1): 1935.

Fredette, B. J. and Landmesser, L. T. (1991b) 'Relationship of primary and secondary myogenesis to fiber type development in embryonic chick muscle', Dev Biol 143(1): 118.

Friday, B. B., Horsley, V. and Pavlath, G. K. (2000) 'Calcineurin activity is required for the initiation of skeletal muscle differentiation', J Cell Biol 149(3): 657-66.

Friday, B. B. and Pavlath, G. K. (2001) 'A calcineurin- and NFAT-dependent pathway regulates Myf5 gene expression in skeletal muscle reserve cells', J Cell Sci 114(Pt 2): 303-10.

Friese, A., Kaltschmidt, J. A., Ladle, D. R., Sigrist, M., Jessell, T. M. and Arber, S. (2009) 'Gamma and alpha motor neurons distinguished by expression of transcription factor Err3', Proc Natl Acad Sci U S A 106(32): 13588-93. 
Furst, D. O., Osborn, M. and Weber, K. (1989) 'Myogenesis in the mouse embryo: differential onset of expression of myogenic proteins and the involvement of titin in myofibril assembly', J Cell Biol 109(2): 517-27.

Gambke, B., Lyons, G. E., Haselgrove, J., Kelly, A. M. and Rubinstein, N. A. (1983) 'Thyroidal and neural control of myosin transitions during development of rat fast and slow muscles', FEBS Lett 156(2): 335-9.

Gensch, N., Borchardt, T., Schneider, A., Riethmacher, D. and Braun, T. (2008) 'Different autonomous myogenic cell populations revealed by ablation of Myf5expressing cells during mouse embryogenesis', Development 135(9): 1597-604.

Giacobini, G., Filogamo, G., Weber, M., Boquet, P. and Changeux, J. P. (1973) 'Effects of a snake alpha-neurotoxin on the development of innervated skeletal muscles in chick embryo', Proc Natl Acad Sci U S A 70(6): 1708-12.

Goldberg, A. L. (1969) 'Protein turnover in skeletal muscle. II. Effects of denervation and cortisone on protein catabolism in skeletal muscle', J Biol Chem 244(12): 3223-9.

Goldman, D., Brenner, H. R. and Heinemann, S. (1988) 'Acetylcholine receptor alpha-, beta-, gamma-, and delta-subunit mRNA levels are regulated by muscle activity', Neuron 1(4): 329-33.

Griesbeck, O., Parsadanian, A. S., Sendtner, M. and Thoenen, H. (1995) 'Expression of neurotrophins in skeletal muscle: quantitative comparison and significance for motoneuron survival and maintenance of function', J Neurosci Res 42(1): 21-33.

Gros, J., Scaal, M. and Marcelle, C. (2004) 'A two-step mechanism for myotome formation in chick', Dev Cell 6(6): 875-82.

Grosberg, A., Kuo, P. L., Guo, C. L., Geisse, N. A., Bray, M. A., Adams, W. J., Sheehy, S. P. and Parker, K. K. (2011) 'Self-organization of muscle cell structure and function', PLoS Comput Biol 7(2): e1001088.

Gunning, P. and Hardeman, E. (1991) 'Multiple mechanisms regulate muscle fiber diversity', FASEB J 5(15): 3064-70. 
Haberland, M., Montgomery, R. L. and Olson, E. N. (2009) 'The many roles of histone deacetylases in development and physiology: implications for disease and therapy', Nat Rev Genet 10(1): 32-42.

Hall, B. K. (1975) 'A simple, single-injection method for inducing long-term paralysis in embryonic chicks, and preliminary observations on growth of the tibia', Anat Rec 181(4): 767-77.

Hamburger, V. (1939) 'The development and innervation of transplanted limb primordia of chick embryos', J. exp. Zool. 7(533-545).

Hamburger, V. (1975) 'Cell death in the development of the lateral motor column of the chick embryo', J Comp Neurol 160(4): 535-46.

Hamburger, V. and Levi-Montalcini, R. (1949) 'Proliferation, differentiation and degeneration in the spinal ganglia of the chick embryo under normal and experimental conditions', J Exp Zool 111(3): 457-501.

Hamburger, V. and Waugh, M. (1940) 'The Primary Development of the Skeleton in Nerveless and Poorly Innervated Limb Transplants of Chick Embryos', Physiol. Zool. 13: 367-382.

Hanson, M. G. and Landmesser, L. T. (2003) 'Characterization of the circuits that generate spontaneous episodes of activity in the early embryonic mouse spinal cord', $J$ Neurosci 23(2): 587-600.

Harris, A. J. (1981) 'Embryonic growth and innervation of rat skeletal muscles. I. Neural regulation of muscle fibre numbers', Philos Trans $R$ Soc Lond B Biol Sci 293(1065): 257-77.

Hasty, P., Bradley, A., Morris, J. H., Edmondson, D. G., Venuti, J. M., Olson, E. N. and Klein, W. H. (1993) 'Muscle deficiency and neonatal death in mice with a targeted mutation in the myogenin gene', Nature 364(6437): 501-6.

Heineke, J., Ruetten, H., Willenbockel, C., Gross, S. C., Naguib, M., Schaefer, A., Kempf, T., Hilfiker-Kleiner, D., Caroni, P., Kraft, T. et al. (2005) 'Attenuation of cardiac 
remodeling after myocardial infarction by muscle LIM protein-calcineurin signaling at the sarcomeric Z-disc', Proc Natl Acad Sci U S A 102(5): 1655-60.

Hollyday, M. and Hamburger, V. (1976) 'Reduction of the naturally occurring motor neuron loss by enlargement of the periphery', J Comp Neurol 170(3): 311-20.

Hollyday, M. and Jacobson, R. D. (1990) 'Location of motor pools innervating chick wing', J Comp Neurol 302(3): 575-88.

Horsley, V., Friday, B. B., Matteson, S., Kegley, K. M., Gephart, J. and Pavlath, G. K. (2001) 'Regulation of the growth of multinucleated muscle cells by an NFATC2dependent pathway', J Cell Biol 153(2): 329-38.

Hory-Lee, F. and Frank, E. (1995) 'The nicotinic blocking agents d-tubocurare and alpha-bungarotoxin save motoneurons from naturally occurring death in the absence of neuromuscular blockade', J Neurosci 15(10): 6453-60.

Houenou, L. J., McManaman, J. L., Prevette, D. and Oppenheim, R. W. (1991) 'Regulation of putative muscle-derived neurotrophic factors by muscle activity and innervation: in vivo and in vitro studies', J Neurosci 11(9): 2829-37.

Houenou, L. J., Pincon-Raymond, M., Garcia, L., Harris, A. J. and Rieger, F. (1990) 'Neuromuscular development following tetrodotoxin-induced inactivity in mouse embryos', J Neurobiol 21(8): 1249-61.

Huang da, W., Sherman, B. T. and Lempicki, R. A. (2009a) 'Bioinformatics enrichment tools: paths toward the comprehensive functional analysis of large gene lists', Nucleic Acids Res 37(1): 1-13.

Huang da, W., Sherman, B. T. and Lempicki, R. A. (2009b) 'Systematic and integrative analysis of large gene lists using DAVID bioinformatics resources', Nat Protoc 4(1): 4457.

Hughes, D. S. and Ontell, M. (1992) 'Morphometric analysis of the developing, murine aneural soleus muscle', Dev Dyn 193(2): 175-84.

Hughes, D. S., Schade, R. R. and Ontell, M. (1992) 'Ablation of the fetal mouse spinal cord: the effect on soleus muscle cytoarchitecture', Dev Dyn 193(2): 164-74. 
Humeau, Y., Doussau, F., Grant, N. J. and Poulain, B. (2000) 'How botulinum and tetanus neurotoxins block neurotransmitter release', Biochimie 82(5): 427-46.

Hunt, C. C. and Kuffler, S. W. (1951) 'Stretch receptor discharges during muscle contraction', J Physiol 113(2-3): 298-315.

Hunt, E. A. (1932) 'The differentiation of chick limb buds in chorio-allontoic grafts, with special reference to the muscles.', J. exp. Zool. 62: 57-91.

Hutcheson, D. A., Zhao, J., Merrell, A., Haldar, M. and Kardon, G. (2009) 'Embryonic and fetal limb myogenic cells are derived from developmentally distinct progenitors and have different requirements for beta-catenin', Genes Dev 23(8): 997-1013.

Inoue, H., Nojima, H. and Okayama, H. (1990) 'High efficiency transformation of Escherichia coli with plasmids', Gene 96(1): 23-8.

Ivanova, A., Signore, M., Caro, N., Greene, N. D., Copp, A. J. and Martinez-Barbera, J. P. (2005) 'In vivo genetic ablation by Cre-mediated expression of diphtheria toxin fragment A', Genesis 43(3): 129-35.

Jahn, R. and Scheller, R. H. (2006) 'SNAREs--engines for membrane fusion', Nat Rev Mol Cell Biol 7(9): 631-43.

Jahn, R. and Sudhof, T. C. (1994) 'Synaptic vesicles and exocytosis', Annu Rev Neurosci 17: $219-46$.

James, N. T. (1976) 'Compensatory muscular hypertrophy in the extensor digitorum longus muscle of the mouse', J Anat 122(Pt 1): 121-31.

Jansen, J. K. and Fladby, T. (1990) 'The perinatal reorganization of the innervation of skeletal muscle in mammals', Prog Neurobiol 34(1): 39-90.

Jessell, T. M. (2000) 'Neuronal specification in the spinal cord: inductive signals and transcriptional codes', Nat Rev Genet 1(1): 20-9.

Jung, H. W. and Wu, W. Y. (1962) 'The contrasting trophic changes of the anterior and posterior latissimus dorsi of the chick following denervation.', Acta physiolo. sin. 25: 304-311. 
Kablar, B., Asakura, A., Krastel, K., Ying, C., May, L. L., Goldhamer, D. J. and Rudnicki, M. A. (1998) 'MyoD and Myf-5 define the specification of musculature of distinct embryonic origin', Biochem Cell Biol 76(6): 1079-91.

Kablar, B. and Belliveau, A. C. (2005) 'Presence of neurotrophic factors in skeletal muscle correlates with survival of spinal cord motor neurons', Dev Dyn 234(3): 659-69. Kablar, B., Krastel, K., Tajbakhsh, S. and Rudnicki, M. A. (2003) 'Myf5 and MyoD activation define independent myogenic compartments during embryonic development', Dev Biol 258(2): 307-18.

Kablar, B., Krastel, K., Ying, C., Asakura, A., Tapscott, S. J. and Rudnicki, M. A. (1997) 'MyoD and Myf-5 differentially regulate the development of limb versus trunk skeletal muscle', Development 124(23): 4729-38.

Kablar, B. and Rudnicki, M. A. (1999) 'Development in the absence of skeletal muscle results in the sequential ablation of motor neurons from the spinal cord to the brain', Dev Biol 208(1): 93-109.

Kablar, B. and Rudnicki, M. A. (2000) 'Skeletal muscle development in the mouse embryo', Histol Histopathol 15(2): 649-56.

Kanehisa, M. and Goto, S. (2000) 'KEGG: kyoto encyclopedia of genes and genomes', Nucleic Acids Res 28(1): 27-30.

Karsch-Mizrachi, I., Travis, M., Blau, H. and Leinwand, L. A. (1989) 'Expression and DNA sequence analysis of a human embryonic skeletal muscle myosin heavy chain gene', Nucleic Acids Res 17(15): 6167-79.

Kassar-Duchossoy, L., Gayraud-Morel, B., Gomes, D., Rocancourt, D., Buckingham, M., Shinin, V. and Tajbakhsh, S. (2004) 'Mrf4 determines skeletal muscle identity in Myf5:Myod double-mutant mice', Nature 431(7007): 466-71.

Kassar-Duchossoy, L., Giacone, E., Gayraud-Morel, B., Jory, A., Gomes, D. and Tajbakhsh, S. (2005) 'Pax3/Pax7 mark a novel population of primitive myogenic cells during development', Genes Dev 19(12): 1426-31. 
Kataoka, H., Murayama, T., Yokode, M., Mori, S., Sano, H., Ozaki, H., Yokota, Y., Nishikawa, S. and Kita, T. (2000) 'A novel snail-related transcription factor Smuc regulates basic helix-loop-helix transcription factor activities via specific E-box motifs', Nucleic Acids Res 28(2): 626-33.

Katz, B. and Miledi, R. (1964) 'The Development of Acetylcholine Sensitivity in NerveFree Segments of Skeletal Muscle', J Physiol 170: 389-96.

Kavalali, E. T., Chung, C., Khvotchev, M., Leitz, J., Nosyreva, E., Raingo, J. and Ramirez, D. M. (2011) 'Spontaneous neurotransmission: an independent pathway for neuronal signaling?', Physiology (Bethesda) 26(1): 45-53.

Kawakami, K. and Shima, A. (1999) 'Identification of the Tol2 transposase of the medaka fish Oryzias latipes that catalyzes excision of a nonautonomous Tol2 element in zebrafish Danio rerio', Gene 240(1): 239-44.

Kegley, K. M., Gephart, J., Warren, G. L. and Pavlath, G. K. (2001) 'Altered primary myogenesis in NFATC3(-/-) mice leads to decreased muscle size in the adult', Dev Biol 232(1): 115-26.

Kelly, A. M. and Zacks, S. I. (1969) 'The histogenesis of rat intercostal muscle', J Cell Biol 42(1): 135-53.

Kojic, S., Medeot, E., Guccione, E., Krmac, H., Zara, I., Martinelli, V., Valle, G. and Faulkner, G. (2004) 'The Ankrd2 protein, a link between the sarcomere and the nucleus in skeletal muscle', J Mol Biol 339(2): 313-25.

Krause, R. M., Hamann, M., Bader, C. R., Liu, J. H., Baroffio, A. and Bernheim, L. (1995) 'Activation of nicotinic acetylcholine receptors increases the rate of fusion of cultured human myoblasts', J Physiol 489 ( Pt 3): 779-90.

Lance-Jones, C. (1982) 'Motoneuron cell death in the developing lumbar spinal cord of the mouse', Brain Res 256(4): 473-9.

Landmesser, L. (1978) 'The development of motor projection patterns in the chick hind limb', J Physiol 284: 391-414. 
Landmesser, L. T. and Szente, M. (1986) 'Activation patterns of embryonic chick hindlimb muscles following blockade of activity and motoneurone cell death', J Physiol 380: $157-74$.

Lassar, A. B., Thayer, M. J., Overell, R. W. and Weintraub, H. (1989) 'Transformation by activated ras or fos prevents myogenesis by inhibiting expression of MyoD1', Cell 58(4): 659-67.

Lee, S. K., Jurata, L. W., Funahashi, J., Ruiz, E. C. and Pfaff, S. L. (2004) 'Analysis of embryonic motoneuron gene regulation: derepression of general activators function in concert with enhancer factors', Development 131(14): 3295-306.

Lee, S. K., Lee, B., Ruiz, E. C. and Pfaff, S. L. (2005) 'Olig2 and Ngn2 function in opposition to modulate gene expression in motor neuron progenitor cells', Genes Dev 19(2): 282-94.

Lei, Fu and Ip (2012) 'Molecular mechanisms underlying maturation and maintenance of the vertebrate neuromuscular junction', Trends in Neuroscience 35(7).

Lepper, C., Partridge, T. A. and Fan, C. M. (2011) 'An absolute requirement for Pax7positive satellite cells in acute injury-induced skeletal muscle regeneration', Development 138(17): 3639-46.

Li, H., Cook, J. D., Terry, M., Spitzer, N. C. and Ferrari, M. B. (2004) 'Calcium transients regulate patterned actin assembly during myofibrillogenesis', Dev Dyn 229(2): 231-42.

Li, S., Czubryt, M. P., McAnally, J., Bassel-Duby, R., Richardson, J. A., Wiebel, F. F., Nordheim, A. and Olson, E. N. (2005) 'Requirement for serum response factor for skeletal muscle growth and maturation revealed by tissue-specific gene deletion in mice', Proc Natl Acad Sci U S A 102(4): 1082-7.

Lin, W., Burgess, R. W., Dominguez, B., Pfaff, S. L., Sanes, J. R. and Lee, K. F. (2001) 'Distinct roles of nerve and muscle in postsynaptic differentiation of the neuromuscular synapse', Nature 410(6832): 1057-64. 
Loughna, P. T., Mason, P., Bayol, S. and Brownson, C. (2000) 'The LIM-domain protein FHL1 (SLIM 1) exhibits functional regulation in skeletal muscle', Mol Cell Biol Res Commun 3(3): 136-40.

Lyons, G. E., Schiaffino, S., Sassoon, D., Barton, P. and Buckingham, M. (1990) 'Developmental regulation of myosin gene expression in mouse cardiac muscle', J Cell Biol 111(6 Pt 1): 2427-36.

Macpherson, P. C., Wang, X. and Goldman, D. (2011) 'Myogenin regulates denervation-dependent muscle atrophy in mouse soleus muscle', J Cell Biochem 112(8): 2149-59.

Madaro, L., Pelle, A., Nicoletti, C., Crupi, A., Marrocco, V., Bossi, G., Soddu, S. and Bouche, M. (2012) 'PKC theta ablation improves healing in a mouse model of muscular dystrophy', PLoS One 7(2): e31515.

Manuel, M. and Zytnicki, D. (2011) 'Alpha, beta and gamma motoneurons: functional diversity in the motor system's final pathway', J Integr Neurosci 10(3): 243-76.

Marquardt, T. and Pfaff, S. L. (2001) 'Cracking the transcriptional code for cell specification in the neural tube', Cell 106(6): 651-4.

Massari, M. E. and Murre, C. (2000) 'Helix-loop-helix proteins: regulators of transcription in eucaryotic organisms', Mol Cell Biol 20(2): 429-40.

Maxwell, I. H., Maxwell, F. and Glode, L. M. (1986) 'Regulated expression of a diphtheria toxin A-chain gene transfected into human cells: possible strategy for inducing cancer cell suicide', Cancer Res 46(9): 4660-4.

McGeachie, J. K. (1989) 'Sustained cell proliferation in denervated skeletal muscle of mice', Cell Tissue Res 257(2): 455-7.

McLennan, I. S. (1983a) 'The development of the pattern of innervation in chicken hindlimb muscles: evidence for specification of nerve-muscle connections', Dev Biol 97(1): 229-38.

McLennan, I. S. (1983b) 'Neural dependence and independence of myotube production in chicken hindlimb muscles', Dev Biol 98(2): 287-94. 
McLennan, I. S. (1994) 'Neurogenic and myogenic regulation of skeletal muscle formation: a critical re-evaluation', Prog Neurobiol 44(2): 119-40.

McLennan, I. S. (1996) 'Degenerating and regenerating skeletal muscles contain several subpopulations of macrophages with distinct spatial and temporal distributions', $J$ Anat 188 ( Pt 1): 17-28.

McMahon, J., Takada S, Z. L., CM, F., RM, H. and AP., M. (1998) 'Noggin-mediated antagonism of BMP signaling is required for growth and patterning of the neural tube and somite.', Genes Dev 15(12): 1438-52.

Merlie, J. P., Mudd, J., Cheng, T. C. and Olson, E. N. (1994) 'Myogenin and acetylcholine receptor alpha gene promoters mediate transcriptional regulation in response to motor innervation', J Biol Chem 269(4): 2461-7.

Messina, G., Biressi, S., Monteverde, S., Magli, A., Cassano, M., Perani, L., Roncaglia, E., Tagliafico, E., Starnes, L., Campbell, C. E. et al. (2010) 'Nfix regulates fetal-specific transcription in developing skeletal muscle', Cell 140(4): 554-66.

Mi, H., Dong, Q., Muruganujan, A., Gaudet, P., Lewis, S. and Thomas, P. D. (2010) 'PANTHER version 7: improved phylogenetic trees, orthologs and collaboration with the Gene Ontology Consortium', Nucleic Acids Res 38(Database issue): D204-10.

Mi, H., Muruganujan, A. and Thomas, P. D. (2012) 'PANTHER in 2013: modeling the evolution of gene function, and other gene attributes, in the context of phylogenetic trees', Nucleic Acids Res.

Misgeld, T., Burgess, R. W., Lewis, R. M., Cunningham, J. M., Lichtman, J. W. and Sanes, J. R. (2002) 'Roles of neurotransmitter in synapse formation: development of neuromuscular junctions lacking choline acetyltransferase', Neuron 36(4): 635-48.

Mok, G. F. and Sweetman, D. (2011) 'Many routes to the same destination: lessons from skeletal muscle development', Reproduction 141(3): 301-12.

Moody, W. J. and Bosma, M. M. (2005) 'Ion channel development, spontaneous activity, and activity-dependent development in nerve and muscle cells', Physiol Rev 85(3): 883-941. 
Moraczewski, J., Nowotniak, A., Wrobel, E., Castagna, M., Gautron, J. and Martelly, I. (2002) 'Differential changes in protein kinase C associated with regeneration of rat extensor digitorum longus and soleus muscles', Int J Biochem Cell Biol 34(8): 938-49.

Moresi, V., Williams, A. H., Meadows, E., Flynn, J. M., Potthoff, M. J., McAnally, J., Shelton, J. M., Backs, J., Klein, W. H., Richardson, J. A. et al. (2010) 'Myogenin and class II HDACs control neurogenic muscle atrophy by inducing E3 ubiquitin ligases', Cell 143(1): 35-45.

Moulik, M., Vatta, M., Witt, S. H., Arola, A. M., Murphy, R. T., McKenna, W. J., Boriek, A. M., Oka, K., Labeit, S., Bowles, N. E. et al. (2009) 'ANKRD1, the gene encoding cardiac ankyrin repeat protein, is a novel dilated cardiomyopathy gene', J Am Coll Cardiol 54(4): 325-33.

Murphy, M., JA, L., SJ, M., DA, H. and G., K. (2011) 'Satellite cells, connective tissue fibroblasts and their interactions are crucial for muscle regeneration', Development 138(17): 3625-37.

Murre, C., McCaw, P. S., Vaessin, H., Caudy, M., Jan, L. Y., Jan, Y. N., Cabrera, C. V., Buskin, J. N., Hauschka, S. D., Lassar, A. B. et al. (1989) 'Interactions between heterologous helix-loop-helix proteins generate complexes that bind specifically to a common DNA sequence', Cell 58(3): 537-44.

Musaro, A., McCullagh, K. J., Naya, F. J., Olson, E. N. and Rosenthal, N. (1999) 'IGF-1 induces skeletal myocyte hypertrophy through calcineurin in association with GATA2 and NF-ATc1', Nature 400(6744): 581-5.

Myhre, J. L. and Pilgrim, D. B. (2012) 'At the Start of the Sarcomere: A Previously Unrecognized Role for Myosin Chaperones and Associated Proteins during Early Myofibrillogenesis', Biochem Res Int 2012: 712315.

Nabeshima, Y., Hanaoka, K., Hayasaka, M., Esumi, E., Li, S., Nonaka, I. and Nabeshima, Y. (1993) 'Myogenin gene disruption results in perinatal lethality because of severe muscle defect', Nature 364(6437): 532-5. 
Novitch, B. G., Chen, A. I. and Jessell, T. M. (2001) 'Coordinate regulation of motor neuron subtype identity and pan-neuronal properties by the bHLH repressor Olig2', Neuron 31(5): 773-89.

O'Leary, M. F., Vainshtein, A., Carter, H. N., Zhang, Y. and Hood, D. A. (2012) 'Denervation-induced mitochondrial dysfunction and autophagy in skeletal muscle of apoptosis-deficient animals', Am J Physiol Cell Physiol 303(4): C447-54.

O'Rourke, J. R., Georges, S. A., Seay, H. R., Tapscott, S. J., McManus, M. T., Goldhamer, D. J., Swanson, M. S. and Harfe, B. D. (2007) 'Essential role for Dicer during skeletal muscle development', Dev Biol 311(2): 359-68.

Ohno, K., Tsujino, A., Brengman, J. M., Harper, C. M., Bajzer, Z., Udd, B., Beyring, R., Robb, S., Kirkham, F. J. and Engel, A. G. (2001) 'Choline acetyltransferase mutations cause myasthenic syndrome associated with episodic apnea in humans', Proc Natl Acad Sci U S A 98(4): 2017-22.

Oki, S., Desaki, J., Matsuda, Y., Okumura, H. and Shibata, T. (1995) 'Capillaries with fenestrae in the rat soleus muscle after experimental limb immobilization', J Electron Microsc (Tokyo) 44(5): 307-10.

Olson, E. N., Arnold, H. H., Rigby, P. W. and Wold, B. J. (1996) 'Know your neighbors: three phenotypes in null mutants of the myogenic bHLH gene MRF4', Cell 85(1): 1-4.

Ontell, M., Hughes, D. and Bourke, D. (1988) 'Morphometric analysis of the developing mouse soleus muscle', Am J Anat 181(3): 279-88.

Ontell, M. and Kozeka, K. (1984) 'Organogenesis of the mouse extensor digitorum logus muscle: a quantitative study', Am J Anat 171(2): 149-61.

Oppenheim, R. W. (1986) 'The absence of significant postnatal motoneuron death in the brachial and lumbar spinal cord of the rat', J Comp Neurol 246(2): 281-6.

Oppenheim, R. W. (1989) 'The neurotrophic theory and naturally occurring motoneuron death', Trends Neurosci 12(7): 252-5.

Oppenheim, R. W. (1991) 'Cell death during development of the nervous system', Annu Rev Neurosci 14: 453-501. 
Oppenheim, R. W., Houenou, L., Pincon-Raymond, M., Powell, J. A., Rieger, F. and Standish, L. J. (1986) 'The development of motoneurons in the embryonic spinal cord of the mouse mutant, muscular dysgenesis (mdg/mdg): survival, morphology, and biochemical differentiation', Dev Biol 114(2): 426-36.

Ordahl, C. P. and Williams, B. A. (1998) 'Knowing chops from chuck: roasting myoD redundancy', Bioessays 20(5): 357-62.

Ott, M. O., Bober, E., Lyons, G., Arnold, H. and Buckingham, M. (1991) 'Early expression of the myogenic regulatory gene, myf-5, in precursor cells of skeletal muscle in the mouse embryo', Development 111(4): 1097-107.

Palmiter, R. D., Behringer, R. R., Quaife, C. J., Maxwell, F., Maxwell, I. H. and Brinster, R. L. (1987) 'Cell lineage ablation in transgenic mice by cell-specific expression of a toxin gene', Cell 50(3): 435-43.

Pardo, J. V., Siliciano, J. D. and Craig, S. W. (1983) 'A vinculin-containing cortical lattice in skeletal muscle: transverse lattice elements ("costameres") mark sites of attachment between myofibrils and sarcolemma', Proc Natl Acad Sci U S A 80(4): 100812.

Patapoutian, A., Yoon, J. K., Miner, J. H., Wang, S., Stark, K. and Wold, B. (1995) 'Disruption of the mouse MRF4 gene identifies multiple waves of myogenesis in the myotome', Development 121(10): 3347-58.

Perez-Ruiz, A., Gnocchi, V. F. and Zammit, P. S. (2007) 'Control of Myf5 activation in adult skeletal myonuclei requires ERK signalling', Cell Signal 19(8): 1671-80.

Pfaff, S. L., Mendelsohn, M., Stewart, C. L., Edlund, T. and Jessell, T. M. (1996) 'Requirement for LIM homeobox gene Isl1 in motor neuron generation reveals a motor neuron-dependent step in interneuron differentiation', Cell 84(2): 309-20.

Phillips, W. D. and Bennett, M. R. (1984) 'Differentiation of fiber types in wing muscles during embryonic development: effect of neural tube removal', Dev Biol 106(2): 457-68. Pittman, R. and Oppenheim, R. W. (1979) 'Cell death of motoneurons in the chick embryo spinal cord. IV. Evidence that a functional neuromuscular interaction is 
involved in the regulation of naturally occurring cell death and the stabilization of synapses', J Comp Neurol 187(2): 425-46.

Plant, P. J., Bain, J. R., Correa, J. E., Woo, M. and Batt, J. (2009) 'Absence of caspase-3 protects against denervation-induced skeletal muscle atrophy', J Appl Physiol 107(1): 224-34.

Pownall, M. E., Gustafsson, M. K. and Emerson, C. P., Jr. (2002) 'Myogenic regulatory factors and the specification of muscle progenitors in vertebrate embryos', Annu Rev Cell Dev Biol 18: 747-83.

Rana, Z. A., Gundersen, K. and Buonanno, A. (2009) 'The ups and downs of gene regulation by electrical activity in skeletal muscles', J Muscle Res Cell Motil 30(7-8): 25560.

Rawls, A., JH, M., M, R., T, B., HH, A., WH, K. and EN., O. (1995) 'Myogenin's functions do not overlap with those of MyoD or Myf-5 during mouse embryogenesis.', Dev Biol. 172(1): 37-50.

Relaix, F., Rocancourt, D., Mansouri, A. and Buckingham, M. (2005) 'A Pax3/Pax7dependent population of skeletal muscle progenitor cells', Nature 435(7044): 948-53.

Reynolds, E. S. (1963) 'The use of lead citrate at high $\mathrm{pH}$ as an electron-opaque stain in electron microscopy', J Cell Biol 17: 208-12.

Rhodes, S. J. and Konieczny, S. F. (1989) 'Identification of MRF4: a new member of the muscle regulatory factor gene family', Genes Dev 3(12B): 2050-61.

Richardson, W. D., Smith, H. K., Sun, T., Pringle, N. P., Hall, A. and Woodruff, R. (2000) 'Oligodendrocyte lineage and the motor neuron connection', Glia 29(2): 136-42.

Rifenberick, D. H., Gamble, J. G. and Max, S. R. (1973) 'Response of mitochondrial enzymes to decreased muscular activity', Am J Physiol 225(6): 1295-9.

Ross, J. J., Duxson, M. J. and Harris, A. J. (1987) 'Neural determination of muscle fibre numbers in embryonic rat lumbrical muscles', Development 100(3): 395-409.

Rostaing, P., Real, E., Siksou, L., Lechaire, J. P., Boudier, T., Boeckers, T. M., Gertler, F., Gundelfinger, E. D., Triller, A. and Marty, S. (2006) 'Analysis of synaptic ultrastructure 
without fixative using high-pressure freezing and tomography', Eur J Neurosci 24(12): 3463-74.

Rubinstein, N. A. and Kelly, A. M. (1978) 'Myogenic and neurogenic contributions to the development of fast and slow twitch muscles in rat', Dev Biol 62(2): 473-85.

Rudnicki, M. A., Braun, T., Hinuma, S. and Jaenisch, R. (1992) 'Inactivation of MyoD in mice leads to up-regulation of the myogenic HLH gene Myf-5 and results in apparently normal muscle development', Cell 71(3): 383-90.

Rudnicki, M. A., Schnegelsberg, P. N., Stead, R. H., Braun, T., Arnold, H. H. and Jaenisch, R. (1993) 'MyoD or Myf-5 is required for the formation of skeletal muscle', Cell 75(7): 1351-9.

Sakuma, K., Watanabe, K., Sano, M., Uramoto, I., Nakano, H., Li, Y. J., Kaneda, S., Sorimachi, Y., Yoshimoto, K., Yasuhara, M. et al. (2001) 'A possible role for BDNF, NT4 and TrkB in the spinal cord and muscle of rat subjected to mechanical overload, bupivacaine injection and axotomy', Brain Res 907(1-2): 1-19.

Sakuma, K. and Yamaguchi, A. (2011) 'The recent understanding of the neurotrophin's role in skeletal muscle adaptation', J Biomed Biotechnol 2011: 201696.

Sambasivan, R. and Tajbakhsh, S. (2007) 'Skeletal muscle stem cell birth and properties', Semin Cell Dev Biol 18(6): 870-82.

Sanes, J. R. and Lichtman, J. W. (1999) 'Development of the vertebrate neuromuscular junction', Annu Rev Neurosci 22: 389-442.

Sato, Y., Kasai, T., Nakagawa, S., Tanabe, K., Watanabe, T., Kawakami, K. and Takahashi, Y. (2007) 'Stable integration and conditional expression of electroporated transgenes in chicken embryos', Dev Biol 305(2): 616-24.

Schiaffino, S. and Mammucari, C. (2011) 'Regulation of skeletal muscle growth by the IGF1-Akt/PKB pathway: insights from genetic models', Skelet Muscle 1(1): 4.

Schiaffino, S. and Reggiani, C. (1996) 'Molecular diversity of myofibrillar proteins: gene regulation and functional significance', Physiol Rev 76(2): 371-423. 
Schiaffino, S. and Reggiani, C. (2011) 'Fiber types in mammalian skeletal muscles', Physiol Rev 91(4): 1447-531.

Schiaffino, S., Sandri, M. and Murgia, M. (2007) 'Activity-dependent signaling pathways controlling muscle diversity and plasticity', Physiology (Bethesda) 22: 269-78. Schmidt, C., Bladt, F., Goedecke, S., Brinkmann, V., Zschiesche, W., Sharpe, M., Gherardi, E. and Birchmeier, C. (1995) 'Scatter factor/hepatocyte growth factor is essential for liver development', Nature 373(6516): 699-702.

Schoch, S., Deak, F., Konigstorfer, A., Mozhayeva, M., Sara, Y., Sudhof, T. C. and Kavalali, E. T. (2001) 'SNARE function analyzed in synaptobrevin/VAMP knockout mice', Science 294(5544): 1117-22.

Schuetze, S. M. and Role, L. W. (1987) 'Developmental regulation of nicotinic acetylcholine receptors', Annu Rev Neurosci 10: 403-57.

Seale, P., Sabourin, L. A., Girgis-Gabardo, A., Mansouri, A., Gruss, P. and Rudnicki, M. A. (2000) 'Pax7 is required for the specification of myogenic satellite cells', Cell 102(6): 777-86.

Sharma, K., Leonard, A. E., Lettieri, K. and Pfaff, S. L. (2000) 'Genetic and epigenetic mechanisms contribute to motor neuron pathfinding', Nature 406(6795): 515-9.

Sharp, W. W., Terracio, L., Borg, T. K. and Samarel, A. M. (1993) 'Contractile activity modulates actin synthesis and turnover in cultured neonatal rat heart cells', Circ Res 73(1): 172-83.

Shellswell, G. B. (1977) 'The formation of discrete muscles from the chick wing dorsal and ventral muscle masses in the absence of nerves', J Embryol Exp Morphol 41: 269-77.

Shirasaki, R. and Pfaff, S. L. (2002) 'Transcriptional codes and the control of neuronal identity', Annu Rev Neurosci 25: 251-81.

Snider, W. D. and Johnson, E. M., Jr. (1989) 'Neurotrophic molecules', Ann Neurol 26(4): 489-506.

Sohal, G. S. and Holt, R. K. (1980) 'Role of innervation on the embryonic development of skeletal muscle', Cell Tissue Res 210(3): 383-93. 
Sohal, G. S. and Sickles, D. W. (1986) 'Embryonic differentiation of fibre types in normal, paralysed and aneural avian superior oblique muscle', J Embryol Exp Morphol 96: 79-97.

Soriano, P. (1999) 'Generalized lacZ expression with the ROSA26 Cre reporter strain', Nat Genet 21(1): 70-1.

Sparrow, J. C. and Schock, F. (2009) 'The initial steps of myofibril assembly: integrins pave the way', Nat Rev Mol Cell Biol 10(4): 293-8.

Stuerenburg, H. J. and Kunze, K. (1998) 'Tissue nerve growth factor concentrations in neuromuscular diseases', Eur J Neurol 5(5): 487-490.

Sudhof, T. C. and Rothman, J. E. (2009) 'Membrane fusion: grappling with SNARE and SM proteins', Science 323(5913): 474-7.

Sweeney, S. T., Broadie, K., Keane, J., Niemann, H. and O'Kane, C. J. (1995) 'Targeted expression of tetanus toxin light chain in Drosophila specifically eliminates synaptic transmission and causes behavioral defects', Neuron 14(2): 341-51.

Tajbakhsh, S. and Buckingham, M. (2000) 'The birth of muscle progenitor cells in the mouse: spatiotemporal considerations', Curr Top Dev Biol 48: 225-68.

Tajbakhsh, S., Rocancourt, D., Cossu, G. and Buckingham, M. (1997) 'Redefining the genetic hierarchies controlling skeletal myogenesis: Pax-3 and Myf-5 act upstream of MyoD', Cell 89(1): 127-38.

Takebayashi, H., Nabeshima, Y., Yoshida, S., Chisaka, O., Ikenaka, K. and Nabeshima, Y.-i. (2002) 'The Basic Helix-Loop-Helix Factor Olig2 Is Essential for the Development of Motoneuron and Oligodendrocyte Lineages.', Current Biology 12(13): 1157-1163.

Thaler, J., Harrison, K., Sharma, K., Lettieri, K., Kehrl, J. and Pfaff, S. L. (1999) 'Active suppression of interneuron programs within developing motor neurons revealed by analysis of homeodomain factor HB9', Neuron 23(4): 675-87.

Tosney, K. W. and Landmesser, L. T. (1985a) 'Development of the major pathways for neurite outgrowth in the chick hindlimb', Dev Biol 109(1): 193-214. 
Tosney, K. W. and Landmesser, L. T. (1985b) 'Growth cone morphology and trajectory in the lumbosacral region of the chick embryo', J Neurosci 5(9): 2345-58.

Tosney, K. W. and Landmesser, L. T. (1985c) 'Specificity of early motoneuron growth cone outgrowth in the chick embryo', J Neurosci 5(9): 2336-44.

Uchikawa, M. (2008) 'Enhancer analysis by chicken embryo electroporation with aid of genome comparison', Dev Growth Differ 50(6): 467-74.

Vaidya, T. B., Rhodes, S. J., Taparowsky, E. J. and Konieczny, S. F. (1989) 'Fibroblast growth factor and transforming growth factor beta repress transcription of the myogenic regulatory gene MyoD1', Mol Cell Biol 9(8): 3576-9.

Vallstedt, A., Muhr, J., Pattyn, A., Pierani, A., Mendelsohn, M., Sander, M., Jessell, T. M. and Ericson, J. (2001) 'Different levels of repressor activity assign redundant and specific roles to Nkx6 genes in motor neuron and interneuron specification', Neuron 31(5): $743-55$.

Vasyutina, E., Stebler, J., Brand-Saberi, B., Schulz, S., Raz, E. and Birchmeier, C. (2005) 'CXCR4 and Gab1 cooperate to control the development of migrating muscle progenitor cells', Genes Dev 19(18): 2187-98.

Ventadour, S. and Attaix, D. (2006) 'Mechanisms of skeletal muscle atrophy', Curr Opin Rheumatol 18(6): 631-5.

Viguie, C. A., Lu, D. X., Huang, S. K., Rengen, H. and Carlson, B. M. (1997) 'Quantitative study of the effects of long-term denervation on the extensor digitorum longus muscle of the rat', Anat Rec 248(3): 346-54.

Wang, H., Bonnet, A., Delfini, M. C., Kawakami, K., Takahashi, Y. and Duprez, D. (2011a) 'Stable, conditional, and muscle-fiber-specific expression of electroporated transgenes in chick limb muscle cells', Dev Dyn 240(5): 1223-32.

Wang, L., Klein, R., Zheng, B. and Marquardt, T. (2011b) 'Anatomical coupling of sensory and motor nerve trajectory via axon tracking', Neuron 71(2): 263-77. 
Washabaugh, C. H., Ontell, M. P., Shand, S. H., Bradbury, N., Kant, J. A. and Ontell, M. (2007) 'Neuronal control of myogenic regulatory factor accumulation in fetal muscle', Dev Dyn 236(3): 732-45.

Williams, A. H., Valdez, G., Moresi, V., Qi, X., McAnally, J., Elliott, J. L., Bassel-Duby, R., Sanes, J. R. and Olson, E. N. (2009) 'MicroRNA-206 delays ALS progression and promotes regeneration of neuromuscular synapses in mice', Science 326(5959): 1549-54. Wilson, S. J. and Harris, A. J. (1993) 'Formation of myotubes in aneural rat muscles', Dev Biol 156(2): 509-18.

Wu, H., Xiong, W. C. and Mei, L. (2010) 'To build a synapse: signaling pathways in neuromuscular junction assembly', Development 137(7): 1017-33.

Yamamoto, M., Wada, N., Kitabatake, Y., Watanabe, D., Anzai, M., Yokoyama, M., Teranishi, Y. and Nakanishi, S. (2003) 'Reversible suppression of glutamatergic neurotransmission of cerebellar granule cells in vivo by genetically manipulated expression of tetanus neurotoxin light chain', J Neurosci 23(17): 6759-67.

Yampolsky, P., Gensler, S., McArdle, J. and Witzemann, V. (2008) 'AChR channel conversion and AChR-adjusted neuronal survival during embryonic development', Mol Cell Neurosci 37(3): 634-45.

Yao, H. H., Whoriskey, W. and Capel, B. (2002) 'Desert Hedgehog/Patched 1 signaling specifies fetal Leydig cell fate in testis organogenesis', Genes Dev 16(11): 1433-40.

Yoshihara, M., Ueda, A., Zhang, D., Deitcher, D. L., Schwarz, T. L. and Kidokoro, Y. (1999) 'Selective effects of neuronal-synaptobrevin mutations on transmitter release evoked by sustained versus transient Ca2+ increases and by cAMP', J Neurosci 19(7): 2432-41.

Yusuf, F., Rehimi, R., Morosan-Puopolo, G., Dai, F., Zhang, X. and Brand-Saberi, B. (2006) 'Inhibitors of CXCR4 affect the migration and fate of CXCR4+ progenitors in the developing limb of chick embryos', Dev Dyn 235(11): 3007-15. 
Yvert, B., Branchereau, P. and Meyrand, P. (2004) 'Multiple spontaneous rhythmic activity patterns generated by the embryonic mouse spinal cord occur within a specific developmental time window', J Neurophysiol 91(5): 2101-9.

Zelena, J. (1976) 'The role of sensory innervation in the development of mechanoreceptors', Prog Brain Res 43: 59-64.

Zelena, J. and Soukup, T. (1974) 'The differentiation of intrafusal fibre types in rat muscle spindles after motor denervation', Cell Tissue Res 153(1): 115-36.

Zhang, M. and McLennan, S. (1995) 'During secondary myotube formation, primary myotubes preferentially absorb new nuclei at their ends.', Dev Dyn 204(2): 168-77.

Zhang, W., Behringer, R. R. and Olson, E. N. (1995) 'Inactivation of the myogenic bHLH gene MRF4 results in up-regulation of myogenin and rib anomalies', Genes Dev 9(11): 1388-99.

Zhang, Y., Narayan, S., Geiman, E., Lanuza, G. M., Velasquez, T., Shanks, B., Akay, T., Dyck, J., Pearson, K., Gosgnach, S. et al. (2008) 'V3 spinal neurons establish a robust and balanced locomotor rhythm during walking', Neuron 60(1): 84-96.

http://rsbweb.nih.gov/ij/

http://primer3.sourceforge.net 


\section{Acknowledgements}

I would like to thank my supervisor Dr. Till Marquardt for warmly accepting me into his lab on a cold winter day, for the opportunities and support he has kindly provided, and for the patience he has shown me. Most importantly, thank you for the scientific-training that has helped me to advance as a researcher.

I would also like to express my gratitude towards my thesis committee members, Prof. Dr. Klaus-Armin Nave and Prof. Dr. Tomas Pieler for their insights and suggestions that have helped shaped my project. My thanks also goes to the extended thesis committee members, Prof. Dr. Ernst Wimmer, Prof. Dr. Silvio Rizzoli and Prof. Dr. Dr. Hannelore Ehrenreich for taking the time off to evaluate my work.

The IMPRS Neurosciences program and its coordinators, Prof. Dr. Michael Hörner and Sandra Drube deserve special thanks because they are the reason why I was able to further my studies in Germany and gained a great experience while at it.

I wish to acknowledge my collaborators, Dr. Lars Wittler, Dr. Phillip Grote, Dr. Christoph Wrede and Prof. Martyn Goulding who have helped this project in one way or another.

To all past and present members of Developmental Neurobiology Laboratory, I consider myself fortunate to have known all of you as colleagues and friends. Thank you Dr. Liang Wang, Dr. Daniel Müller, Dr. David Herholz, Dr. Pitchaiah Cherukuri, Camille Lancelin, Beate Veith and Nidhi Subhashini for your kind actions and readiness to help out. Also thanks to my coffee mates: Dr. Lukas Cyganek and Heiko Röhse for your goofiness that never fails to cheer me up. I'm also grateful to Dr. Tsung-I Lee, best benchmate who went through the highs and lows of PhD life with 
me-JDLR! Lastly, Danke schön to my Deutsch Lehrerin Alexandra Klusowski for her liveliness and constant support.

To my friends here and abroad, you know I have alot to be thankful for. I would not be where I am now without the love and encouragement from my parents and Peggy. Thank you for always believing in me, even when I sometimes doubt myself- I hope I've made you proud.

To Nattipong, thank you for your unlimited patience and love that I wouldn't trade for anything in the world. 


\title{
9. Curriculum Vitae
}

\section{CHOR HOON POH}

\author{
Address: \\ Im Hassel 13, 37077 Göttingen, Germany \\ Email: \\ Marital status: \\ cpoh1@gwdg.de \\ Nationality: \\ Married \\ Date of birth: \\ Singaporean \\ Place of birth: \\ 20 $0^{\text {th }}$ February 1985 \\ Bangkok, Thailand
}

\section{EDUCATIONAL HISTORY}

- 04/2009- Present

- 09/2007-03/2009

- $09 / 2002-08 / 2006$
International Max Planck Research School, GeorgAugust-University of Göttingen, Germany

Ph.D (Neurosciences)

Project title: The role of innervation during embryonic myogenesis: what molecular genetics tells International Max Planck Research School, GeorgAugust-University of Göttingen, Germany M.Sc. (Neurosciences)

Project title: Electrophysiological characterization of postnatal development of perisomatic inhibition to pyramidal neurons in prefrontal cortex of mouse Mahidol University International College, Thailand B.Sc. (Biological Sciences, First Class Honours) Project title: Transfer of Arylsulfatase-A (AS-A) to mouse sperm surface via epididymosomes during epididymal residence

Stipend of the Excellence Foundation for the Promotion of the Max Planck Society

GGNB bridging fellowship (GGNB- Göttingen Graduate School for Neurosciences and Molecular Biosciences)

UMG PhD scholarship (UMG- University Medicine Göttingen) 